\title{
Hydrogels Based on Amphiphilic PEG Star Block Copolýmers
}




\section{HYDROGELS BASED ON AMPHIPHILIC PEG STAR BLOCK COPOLYMERS}

Sytze Buwalda 


\section{Committee}

Chairman: Prof. Dr. G. van der Steenhoven University of Twente, the Netherlands

Promotor: Prof. Dr. J. Feijen University of Twente

Co-promotor: Prof. Dr. P. J. Dijkstra Soochow University, China

Members: $\quad$ Prof. Dr. D. W. Grijpma University of Twente

Prof. Dr. Ir. R. G. H. Lammertink University of Twente

Prof. Dr. W. E. Hennink Utrecht University, the Netherlands

Prof. Dr. Ir. J. C. M. van Hest Radboud University Nijmegen, the Netherlands

Prof. Dr. C. Jérôme Université de Liège, Belgium

Dr. C. Forte Istituto di Chimica dei Composti OrganoMetallici,

Consiglio Nazionale delle Ricerche, Pisa, Italy

The research described in this thesis was financially supported by the Dutch Program for Tissue

Engineering (DPTE, project number 6732).

The printing of this thesis was sponsored by the Dutch Society for Biomaterials and Tissue Engineering (NBTE).

Hydrogels based on amphiphilic PEG star block copolymers.

Sytze Buwalda

$\mathrm{PhD}$ thesis, with references; summaries in English and Dutch.

University of Twente, Enschede, the Netherlands.

ISBN 978-90-365-3290-7

DOI http://dx.doi.org/10.3990/1.9789036532907

Copyright (C) 2011 by Sytze Buwalda. All rights reserved.

Printed by Wöhrmann Print Service, Zuthpen, the Netherlands.

Cover: 'Night sky \& stars' by www.psdgraphics.com 


\title{
HYDROGELS BASED ON AMPHIPHILIC PEG STAR BLOCK COPOLYMERS
}

\section{DISSERTATION}

\author{
to obtain \\ the degree of doctor at the University of Twente, \\ on the authority of the rector magnificus, \\ Prof. Dr. H. Brinksma, \\ on account of the decision of the graduation committee, \\ to be publicly defended \\ on Thursday the $8^{\text {th }}$ of December 2011 at 16:45
}

by

\section{Sytze Jan Buwalda}

born on the $21^{\text {st }}$ of August 1981

in Apeldoorn, the Netherlands 
This dissertation has been approved by:

\section{Promotor}

Prof. Dr. J. Feijen

Co-promotor

Prof. Dr. P. J. Dijkstra

Copyright (C) 2011 by Sytze Buwalda.

ISBN 978-90-365-3290-7 
Chapter 1 General introduction

Chapter 2 PEG-PLA block copolymer hydrogels for biomedical applications

Chapter 3 The influence of amide versus ester linkages on the properties of 8-armed PEG-PLA star block copolymer hydrogels

Chapter 4 Stereocomplexed 8-armed PEG-PLA star block copolymer 55 hydrogels

Chapter 5 Self-assembly and photocrosslinking of 8-armed PEG-PTMC star 75 block copolymers

Chapter 6 In situ forming PEG-PLA hydrogels via Michael addition. 95 Mechanical properties, degradation and protein release

Chapter 7 In situ forming stereocomplexed and photocrosslinked PEG-PLA hydrogels. Mechanical properties, degradation and drug release

Chapter 8 PEG-PLLA star block copolymer hydrogels crosslinked by metalligand coordination

Summary

Samenvatting 



\section{Chapter 1}

\section{General introduction}

\subsection{Hydrogels for the controlled delivery of biologically active agents}

Hydrogels are polymer networks based on hydrophilic macromonomers that are able to retain large amounts of water. ${ }^{1}$ They generally exhibit excellent biocompatibility as a result of their high water content. Hydrogels are currently used in a wide range of applications from cosmetics to biomedical products (Figure 1).
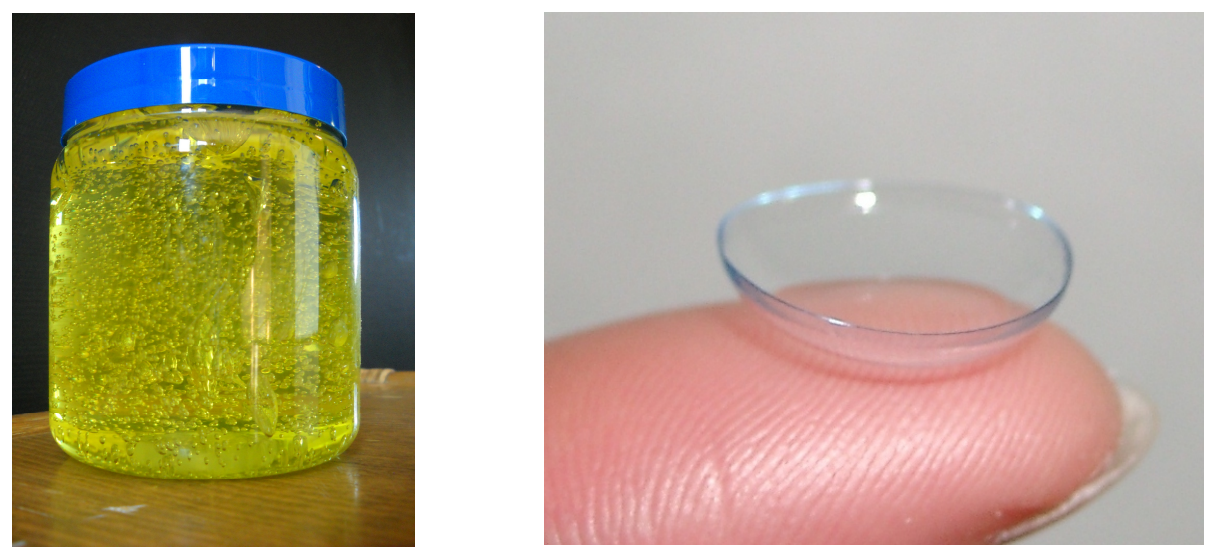

Figure 1. Examples of a hydrogel: hair gel (left) and contact lens (right).

Two classes of hydrogels are distinguished, physically crosslinked hydrogels in which the network structure is maintained by non-covalent interactions and chemically crosslinked hydrogels in which covalent bonds between the macromonomers provide a stable network structure. The gelation in physically crosslinked hydrogels is in many cases reversible and can be controlled with parameters like temperature. In biomedical applications the use of hydrogels that are formed "in situ", indicating that gelation is taking place upon injection, is preferred over pre-made hydrogels since there is no need for surgical procedures. The initial flowing nature of the precursor solution ensures proper shape adaptation and biological components can be incorporated in the hydrogel by simple mixing with the precursor polymer solution (Figure 2$)^{2}$ 


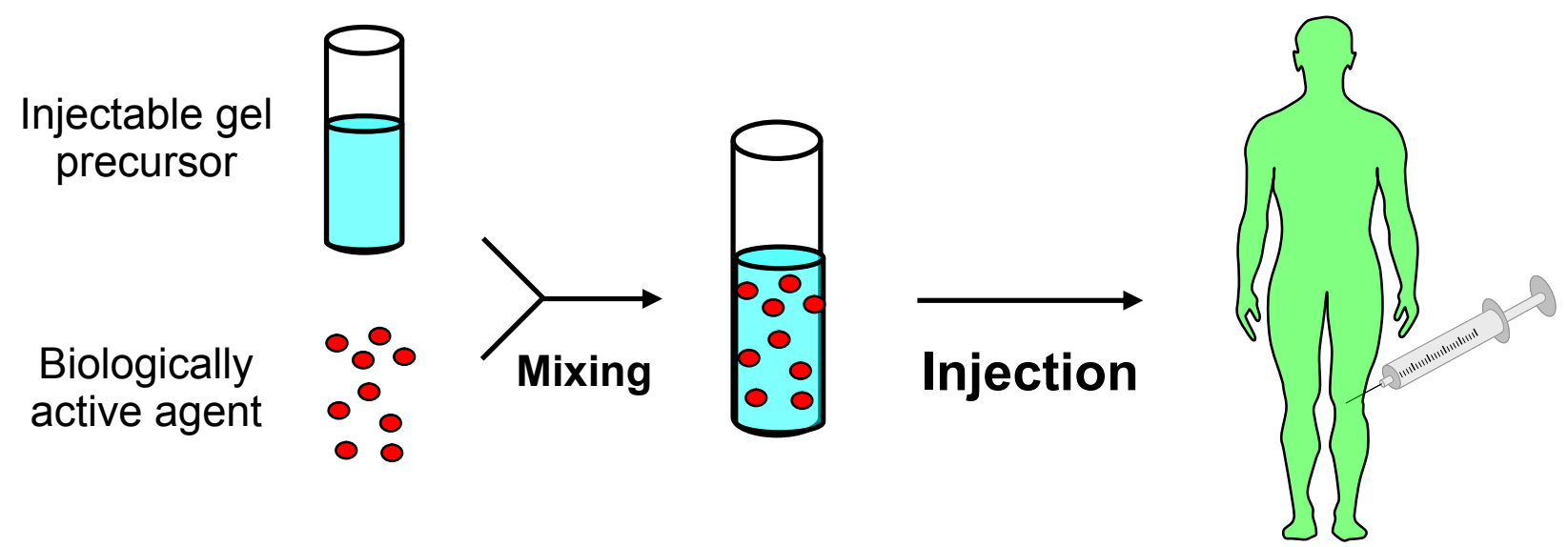

Figure 2. Representation of an in situ forming hydrogel for the delivery of a biologically active agent.

Both natural and synthetic polymers have been applied for the preparation of hydrogels. Amongst the synthetic polymers, poly(ethylene glycol) (PEG) is most widely used as a hydrophilic component. The versatility of PEG macromonomer chemistry and its excellent biocompatibility have initiated the development of numerous physically and chemically crosslinked hydrogel systems for biomedical applications. ${ }^{3}$ To render the systems biodegradable, PEG is frequently used as the hydrophilic component in amphiphilic block copolymers with biodegradable polyesters such as poly(lactide) (PLA) and poly( $\varepsilon$-caprolactone) (PCL) as second hydrophobic components.

Conventional drug delivery approaches such as injection and oral delivery have a number of disadvantages, including poor control of local or systemic drug concentration and the necessity of high initial doses due to dilution effects and drug degradation. Moreover, the effective delivery of many therapeutic agents is challenging because of their poor solubility in biological fluids. These issues can be addressed by using hydrogel based controlled drug delivery systems. As an example, a solution of poly(lactide-co-glycolide)-poly(ethylene glycol)-poly(lactide-co-glycolide) (PLGAPEG-PLGA) triblock copolymer in PBS has become commercially available under the name ReGel ${ }^{\circledR}$. This system acts as a controlled release drug depot that is injected as a liquid and forms a physically crosslinked hydrogel in response to body temperature. A formulation of ReGel® loaded with the hydrophobic anti-cancer drug paclitaxel, called OncoGel ${ }^{\circledR}$, exhibited a sustained release of the drug for approximately $50 \mathrm{~d}$ in vitro (Figure 3$).{ }^{4}$ Intratumoral injections of OncoGel ${ }^{\circledR}$ in mice resulted in a higher concentration of paclitaxel in the tumor compared to intravenously administered drug. As a result, OncoGel®-treated animals showed less drug-related adverse effects and higher survival rates compared to the systemically treated animals. ReGel ${ }^{\circledR}$ also exhibited sustained release kinetics for therapeutic proteins. 


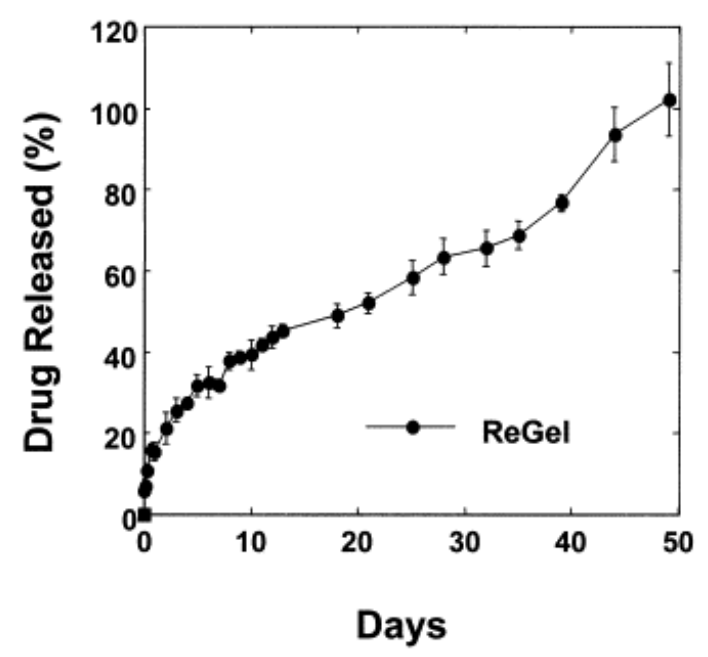

Figure 3. In vitro release of paclitaxel from ReGel®. Reprinted from reference 4. Copyright 2001, with permission from Elsevier.

Most physically and chemically crosslinked hydrogels that have been applied as controlled drug delivery systems are based on linear amphiphilic PEG copolymers. ${ }^{5-8}$ Star block copolymers offer various advantages over linear polymers, such as increased solubility and a higher concentration of end groups that can be used for (bio)functionalization. ${ }^{9}$ However, only a few studies report on drug release from hydrogels which are prepared from star shaped copolymers based on PEG and biodegradable hydrophobic blocks. ${ }^{10,11}$

\subsection{Aim of the study}

The aim of the study described in this thesis was to design and prepare physically or chemically crosslinked injectable hydrogels from PEG-PLA star block copolymers. In this respect, controlled degradation and sufficient mechanical properties of the injectable hydrogels were taken into account in the design. Furthermore the gelation mechanism and the eventual hydrogel degradation mechanism were studied in detail. Their potential application as systems for the controlled delivery of biologically active agents was evaluated.

\subsection{Outline of the thesis}

In this thesis physically and chemically crosslinked hydrogels based on amphiphilic PEG star block copolymers are described. Parts of this thesis have been published elsewhere or have been submitted for publication. ${ }^{12-19}$ In Chapter 2 a literature overview is given on the physical and chemical crosslinking methods that have been applied for the synthesis of PEG based hydrogels for biomedical applications, with emphasis on PEG-PLA block copolymer hydrogels that can be formed in situ. In Chapter 3 the synthesis and thermo-responsive phase behavior of 8-armed PEGPLA star block copolymers linked by an amide group between the PEG core and the PLA blocks 
(PEG-(PLA) 8 ) are described. The physical and mechanical properties of hydrogels prepared from these block copolymers are compared to previously described block copolymers containing an ester linking unit. Moreover, the degradation mechanism of both hydrogel types is investigated in detail. In Chapter 4 stereocomplexed hydrogels prepared from enantiomeric PEG-(PDLA) 8 and PEG$(\mathrm{PLLA})_{8}$ solutions are reported. The physical, mechanical and degradation properties of these systems are discussed together with the release of the model protein lysozyme. Moreover, the temperature dependent formation of stereocomplexes is studied in detail and a gelation mechanism at a macromolecular level is proposed. The synthesis of 8-armed poly(ethylene glycol)poly(trimethylene carbonate) star block copolymer (PEG-(PTMC) 8 ) by metal-free ring opening polymerization of TMC initiated by PEG- $\left(\mathrm{NH}_{2}\right)_{8}$ is described in Chapter 5. A detailed study is conducted towards the self-assembly of PEG-(PTMC) $)_{8}$ in water. Although stable bridging between polymer aggregates, necessary to form a physically crosslinked hydrogel, is disfavored due to the high mobility of the PTMC blocks, interaggregate bridging can be achieved by UV crosslinking of acrylated PEG-(PTMC) 8 . The physical, mechanical and degradation properties of the photocrosslinked PEG-PTMC hydrogel are investigated, as well as its biocompatibility. In Chapter 6 chemically crosslinked hydrogels are reported which are synthesized from PEG-(PLA) $)_{8}$ bearing acrylate end groups and multifunctional PEG thiols through a Michael type addition reaction. Protein release from these systems is studied in relation to their crosslink density and degradation properties. In Chapter 7 PEG-PLA hydrogels are discussed which are formed by physical gelation through stereocomplexation of PEG-(PDLA) 8 and PEG-(PLLA) 8 followed by UV photopolymerization of PLA terminal acrylate groups. To evaluate the potential of these systems for controlled drug delivery, the release properties of the photopolymerized gels are investigated using lysozyme, albumin and rhodamine B as model compounds. In Chapter 8 the feasibility of crosslinking PEG-PLA hydrogels by metal-ligand coordination is explored. The synthesis of pyridine end functionalized PEG-(PLA) 8 (PEG-(PLLA) 8 -py) is described together with its aqueous solution behavior. The physical, mechanical and degradation properties of PEG-(PLLA) 8 -py metallo-hydrogels are compared with those of hydrogels in the absence of metal ions.

\subsection{References}

[1] Peppas, N. A.; Khare, A. R. Adv. Drug Delivery Rev. 1993, 11, 1-35.

[2] Ruel-Gariepy, E.; Leroux, J. C. Eur. J. Pharm. Biopharm. 2004, 58, 409-426.

[3] Lin, C. C.; Anseth, K. S. Pharm. Res. 2009, 26, 631-643.

[4] Zentner, G. M.; Rathi, R.; Shih, C.; McRea, J. C.; Seo, M. H.; Oh, H.; Rhee, B. G.; Mestecky, J.; Moldoveanu, Z.; Morgan, M.; Weitman, S. J. Controlled Release 2001, 72, 203-215.

[5] Jeong, B.; Bae, Y. H.; Lee, D. S.; Kim, S. W. Nature 1997, 388, 860-862.

[6] Vermonden, T.; Jena, S. S.; Barriet, D.; Censi, R.; van der Gucht, J.; Hennink, W. E.; Siegel, R. A. Macromolecules 2010, 43, 782-789.

[7] Lee, W. F.; Cheng, T. S. J. Biomater. Sci., Polym. Ed. 2009, 20, 2023-2037. 
[8] West, J. L.; Hubbell, J. A. React. Polym. 1995, 25, 139-147.

[9] Cameron, D. J. A.; Shaver, M. P. Chem. Soc. Rev. 2011, 40, 1761-1776.

[10] Hiemstra, C.; Zhong, Z.; Van Tomme, S. R.; van Steenbergen, M. J.; Jacobs, J. J. L.; Den Otter, W.; Hennink, W. E.; Feijen, J. J. Controlled Release 2007, 119, 320-327.

[11] Lee, S. J.; Bae, Y.; Kataoka, K.; Kim, D.; Lee, D. S.; Kim, S. C. Polym. J. 2008, 40, $171-$ 176.

[12] Buwalda, S. J.; Dijkstra, P. J.; Calucci, L.; Forte, C.; Feijen, J. Biomacromolecules 2010, 11, 224-232.

[13] Buwalda, S. J.; Perez, L. B.; Teixeira, S.; Calucci, L.; Forte, C.; Feijen, J.; Dijkstra, P. J. Biomacromolecules 2011, 12, 2746-2754.

[14] Calucci, L.; Forte, C.; Buwalda, S. J.; Dijkstra, P. J.; Feijen, J. Langmuir 2010, 26, 12890-12896.

[15] Calucci, L.; Forte, C.; Buwalda, S. J.; Dijkstra, P. J. Macromolecules 2011, 44, 72887295.

[16] Buwalda, S. J.; Dijkstra, P. J.; Feijen, J. J. Controlled Release 2010, 148, e23-e24.

[17] Buwalda, S. J.; Calucci, L.; Forte, C.; Dijkstra, P. J.; Feijen, J. Macromolecules 2011, submitted

[18] Buwalda, S. J.; Dijkstra, P. J.; Feijen, J. Macromol. Biosci. 2011, submitted.

[19] Buwalda, S. J.; De Graaff, M.; Dijkstra, P. J.; Feijen, J. J. Polym. Sci., Part A: Polym. Chem. 2011, submitted. 


\title{
PEG-PLA block copolymer hydrogels for biomedical applications
}

\author{
Sytze J. Buwalda, Pieter J. Dijkstra, and Jan Feijen
}

Department of Polymer Chemistry and Biomaterials, Faculty of Science and Technology, MIRA Institute for Biomedical Technology and Technical Medicine, University of Twente, P.O. Box 217, 7500 AE Enschede, The Netherlands

\subsection{Introduction}

Hydrogels are three-dimensional polymer networks that are able to retain a large amount of water in their swollen state. ${ }^{1}$ After their discovery in the 1960 s by Wichterle and Lim $^{2}$ they were first successfully applied as contact lenses. Later, hydrogels have been frequently used in biomedical areas such as tissue engineering ${ }^{3-5}$ and systems for the controlled delivery of biologically active agents. $^{6-8}$ The popularity of hydrogels in biomedical research is partly related to their excellent biocompatibility. Due to their high water content the properties of hydrogels resemble those of biological tissues. Furthermore, their soft and rubbery nature minimizes inflammatory reactions of the surrounding cells. ${ }^{9}$ The interactions responsible for the water sorption include capillary, osmotic and hydration forces, which are counterbalanced by the forces exerted by the crosslinked polymer chains in resisting expansion. ${ }^{10}$ The equilibrium swollen state depends on the magnitudes of these opposing effects, and determines to a large extent some important properties of the hydrogel, including internal transport and diffusion characteristics, and mechanical strength. Many of these properties are governed not only by the degree of swelling, but also directly by the chemical nature of the polymer network and the network morphology.

Hydrogels are either chemically crosslinked by covalent bonds or physically crosslinked by noncovalent interactions. Both approaches have been used in recent years for the preparation of hydrogels that can be applied under physiological conditions. Important developments in this area are the "in situ" forming hydrogel systems. These are injectable fluids that can be introduced into any tissue, organ or body cavity in a minimally invasive manner prior to gelation. ${ }^{11}$ In situ forming hydrogels offer several advantages over systems that have to be formed into their final shape before implantation. There is no need for surgical procedures and their initially flowing nature ensures proper shape adaptation as well as a good fit with the surrounding tissue.

The polymers that constitute a hydrogel network may either be synthetic, or naturally derived. Commonly used natural polymers include proteins such as fibrin, ${ }^{12}$ collagen $^{13}$ and gelatin. ${ }^{14}$ 
Polysaccharides such as chitosan, ${ }^{15}$ alginate, ${ }^{16}$ dextran ${ }^{17}$ and hyaluronic acid ${ }^{18,19}$ have been applied as well. Naturally derived polymers generally possess a less defined chemical structure compared to synthetic polymers, which may result in less controlled mechanical properties and degradation. Furthermore they may provoke a severe immunological response, accommodate viruses or microbes and their supply from one source may be limited. ${ }^{20}$ The chemical structure of several important synthetic polymers applied in the synthesis of hydrogels is shown in Figure 1. The most widely used synthetic polymer is poly(ethylene glycol) (PEG), which possesses excellent biocompatibility due to its high hydrophilicity. ${ }^{21}$ PEG can be excreted via the renal pathway up to a molecular weight of approximately $30 \mathrm{~kg} / \mathrm{mol}^{22}$ PEG is also often applied as the hydrophilic component in amphiphilic block copolymers. As a hydrophobic component poly(lactide) (PLA) has been used extensively. ${ }^{23}$ This aliphatic polyester shows excellent biocompatibility, has good mechanical properties and degrades by hydrolytic or enzymatic cleavage of the ester linkages and eventually is metabolized into carbon dioxide and water. Other examples of hydrophobic blocks in amphiphilic copolymers include poly(propylene oxide) (PPO), ${ }^{24}$ poly(glycolide) (PGA), poly(3methylglycolide) $(\mathrm{PMG}){ }^{25}$ poly $\left(\delta\right.$-valerolactone) $(\mathrm{PVL}),{ }^{26} \operatorname{poly}(\varepsilon$-caprolactone $)(\mathrm{PCL})^{27}$ and poly(trimethylene carbonate) (PTMC) ${ }^{28}$ as well as corresponding copolymers.

In the following sections several physical and chemical crosslinking methods are discussed which have been applied for the synthesis of PEG based hydrogels for biomedical applications. Emphasis will be placed on PEG-PLA block copolymers that can form hydrogels in situ. 
<smiles>CC(C)(C)CCOC(C)(C)C</smiles>

PEG<smiles>CC(CC(C)(C)C)OC(C)(C)C</smiles>

PPO<smiles>CC(C)(C)COC(=O)C(C)(C)C</smiles>

PGA<smiles>CC(OC(=O)C(C)(C)C)C(C)(C)C</smiles>

PLA<smiles>CC(OC(=O)C(C)(C)C)C(=O)OCC(C)(C)C</smiles>

PMG<smiles>CC(C)(C)CCCCCOC(=O)C(C)(C)C</smiles>

PCL<smiles>CC(C)(C)CCCCOC(=O)C(C)(C)C</smiles>

PVL<smiles>CC(C)(C)CCCOC(=O)OC(C)(C)C</smiles>

PTMC

Figure 1. Molecular formulas of synthetic polymers that are frequently used for the preparation of hydrogels.

\subsection{Physically crosslinked hydrogels}

In physically crosslinked hydrogels a substantial fraction of the polymer chains is involved in the formation of stable interactions. ${ }^{29}$ The interacting chain segments form junction zones, in which a more ordered structure is maintained than in unassociated chain segments. These junction zones behave as crosslink sites thereby forming a network. These crosslinks generally form under mild conditions but can also be easily disrupted by a change in environmental parameters such as temperature or $\mathrm{pH}$.

Thermo-responsive hydrogels. PEO-PPO-PEO triblock copolymers, commercially known as Pluronics (BASF) or Poloxamers (ICI), are widely studied thermo-responsive gel systems. ${ }^{30}$ Aqueous solutions of selected Pluronics exhibit a phase transition from the sol to the gel state generally at low temperatures and from the gel to the sol state at higher temperatures when the concentration is above the critical gel concentration (CGC). Although investigated intensively, the exact gelation mechanism of Pluronic solutions is still being debated. ${ }^{31}$ In general, it is believed that the triblock copolymers form micelles which equilibrate with unimers at low temperatures. As the temperature increases, the equilibrium shifts from unimers to spherical micelles, reducing the number of unassociated unimers in solution, leading to an increase in the micelle volume fraction. 
When the volume fraction of the micelles becomes larger than the maximum packing fraction, a gel is formed. Significant drawbacks of Pluronic hydrogels are their weak mechanical properties and intrinsic instability, which are believed to originate from the weak hydrophobic interactions between PPO blocks. Moreover, these block copolymers are not biodegradable, which prevents the use of high molecular weight materials since they cannot pass the kidney membranes. These drawbacks prompted several researchers to replace the hydrophobic PPO block for a biodegradable polyester block as a basis for thermo-responsive hydrogels. Both ABA type and BAB type copolymers, with A as the PEG block and B the polyester block, have been synthesized using e.g. PLA or PCL as the hydrophobic B block.

The group of Kim synthesized a number of linear AB diblock and ABA triblock copolymers, with A as a hydrophilic PEG block $\left(M_{n}=5 \mathrm{~kg} / \mathrm{mol}\right)$ and B as a hydrophobic PLA block. ${ }^{32,33}$ Diblock copolymers were synthesized by ring opening polymerization of lactide initiated by the hydroxyl group of monomethoxy PEG, while triblock copolymers were prepared by coupling the diblock copolymers with monomethoxy PEG using hexamethylene diisocyanate (HMDI). At low concentrations, aqueous solutions of these copolymers consist of micelles, as indicated by dye solubilization experiments. This micelle formation was ascribed to hydrophobic interactions of the polyester blocks. At higher concentrations and depending on the temperature, the aqueous polymer solutions formed gels due to association of the micelles. The gels transformed into sols when the temperature was increased, which was attributed to shrinkage of the PEG corona and accompanying micelle collapse, or by a change in micellar structure from a spherical to a cylindrical shape. In comparison with diblock copolymers possessing the same PEG content and PEG molecular weight, triblock copolymers generally yielded hydrogels at lower polymer concentrations (Figure 2). The thermo-responsive behavior could be tuned via the hydrophilic/hydrophobic balance, the block length, and the stereoregularity of the PLA block. For PEG-PLLA-PEG triblock copolymers, the CGC decreased from 20 to $12 \mathrm{w} / \mathrm{v} \%$ upon increase of the PLLA block length from 2 to $5 \mathrm{~kg} / \mathrm{mol}$. 

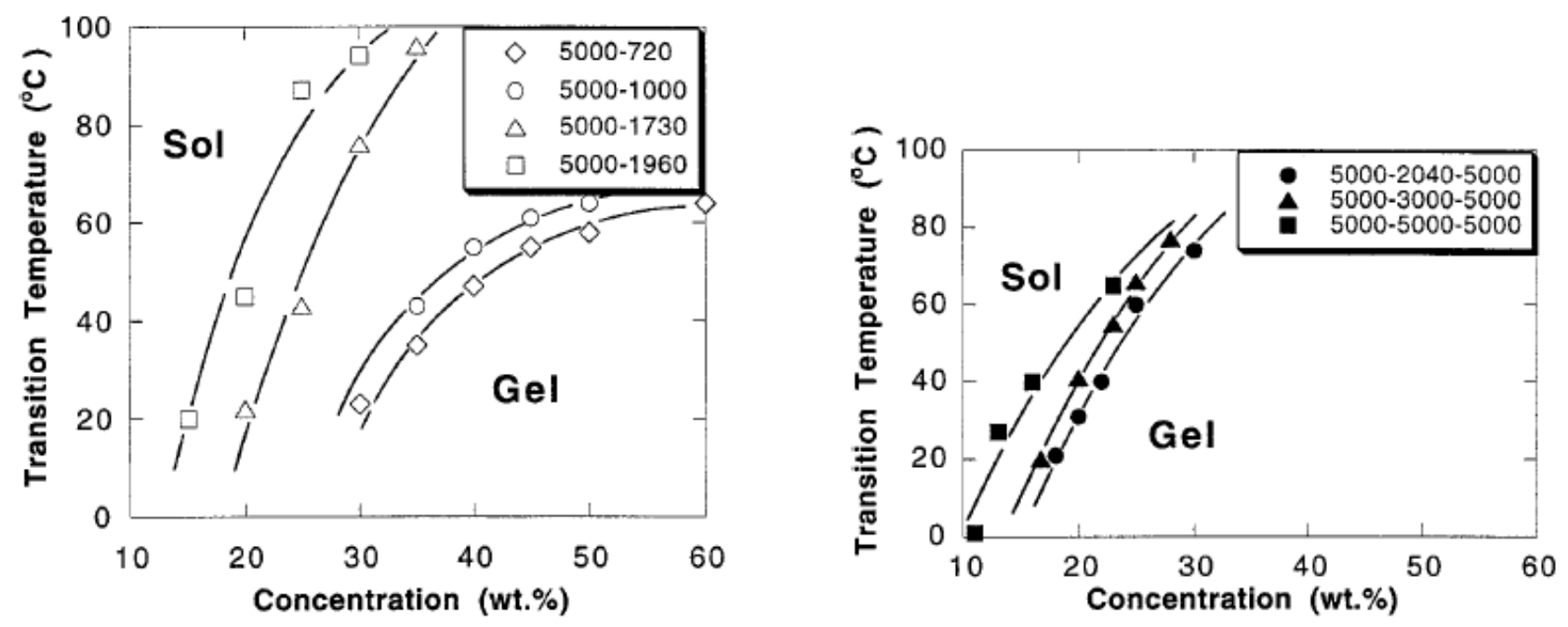

Figure 2. Gel-sol transition curves of PEG-PLLA diblock (left) and PEG-PLLA-PEG triblock (right) copolymers. ${ }^{32}$ The numbers indicate the molecular weight of each block. Reprinted with permission from John Wiley \& Sons, Inc.

Block copolymers with an inverted structure (BAB) can be easily prepared by ring opening polymerization of lactide initiated by the hydroxyl groups of PEG. At room temperature the CGC of triblock copolymers with a PEG $\mathrm{M}_{\mathrm{n}}$ of $12.5 \mathrm{~kg} / \mathrm{mol}$ decreased significantly from 80 to $15 \mathrm{w} / \mathrm{v} \%$ upon an increase of the PLA block length from 10 to 15 lactyl units, showing a much stronger effect of the PLA block length on the gelation behavior in comparison with ABA type triblock copolymers. $^{34} \mathrm{Li}$ et al. investigated the degradation behavior of PLA-PEG-PLA triblock copolymers of high molecular weight (total $\mathrm{M}_{\mathrm{n}} 45-75 \mathrm{~kg} / \mathrm{mol}$ ). ${ }^{35}$ Degradation was initially very fast with significant weight loss. The PLA/PEG ratio of the remaining material increased rapidly, indicating the release of PEG-rich segments. In a second phase, the degradation rate slowed down because of the high PLA content of the remaining material. The presence of proteinase K strongly accelerated the degradation rate of the hydrogels, showing that the enzyme was able to penetrate inside and attack the PLA domains. The PLA/PEG ratio in the residual hydrogel was found to increase as in the case of hydrolytic degradation.

Tew et al. investigated the effect of PLA stereoregularity on the mechanical properties and the microstructure of PLA-PEG-PLA triblock copolymer hydrogels. They showed that polymers with stereoregular PLLA blocks yield hydrogels with a significantly higher elastic modulus compared to polymers with stereo-irregular PDLLA blocks due to the formation of stiff, crystalline PLLA crystals in the gel phase. ${ }^{36}$ Small-angle neutron scattering (SANS) suggested that the copolymers with PDLLA blocks form flower-like micelles in dilute solutions (Figure 3). ${ }^{37}$ With an increase in polymer concentration, the hydrophobic end groups associated with the neighboring micelles to form a network of spherical micelles. The copolymers with PLLA blocks, on the other hand, formed non-spherical, lamellar micelles (Figure 3). The PLLA end blocks associated between 
neighboring lamellae to form a network structure of randomly oriented lamellar micelles at higher concentrations, leading to the formation of stiff hydrogels with a high elastic modulus.
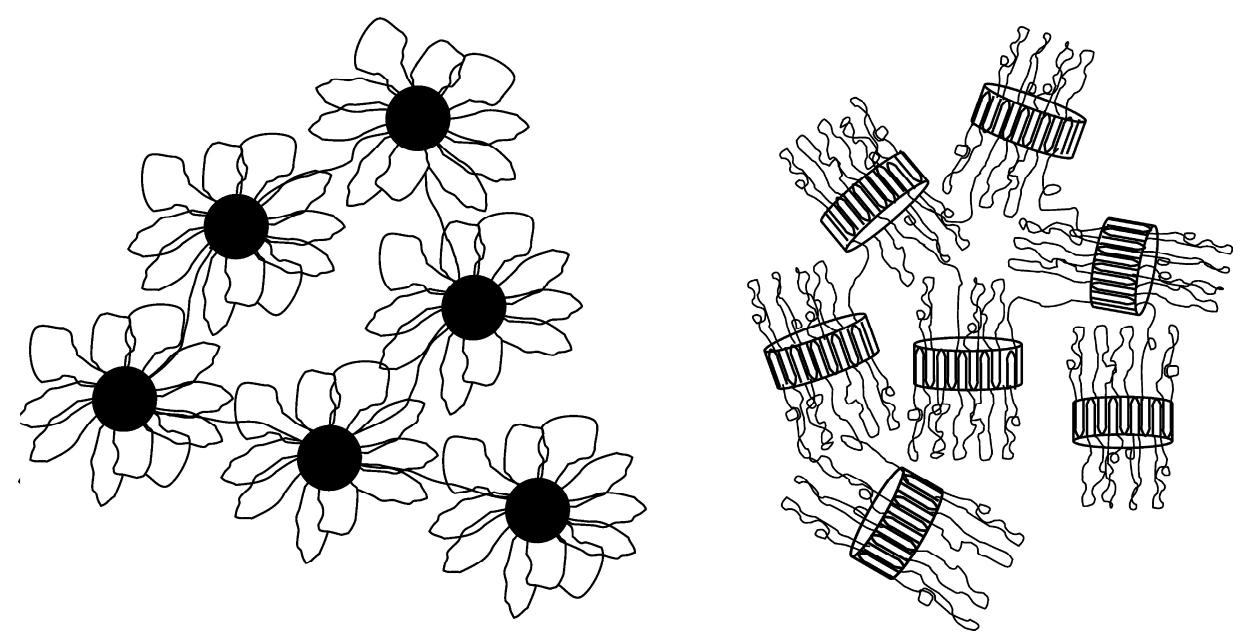

Figure 3. Representation of the networks that are formed when neighbouring micelles of PLAPEG-PLA triblock copolymers associate. Flower-like micelles with an amorphous PDLLA core (left) and lamellar micelles with a semi-crystalline PLLA core (right). Reprinted with permission from reference 37. Copyright 2008 American Chemical Society.

Hydrogels based on alternating multiblock copolymers of PEG and PLA have also been reported. The polymers were synthesized by coupling PEG diols to PLA diols using succinic anhydride ${ }^{38,39}$ or by coupling hydroxyl end functionalized PLA-PEG-PLA triblock copolymers using adipoyl chloride. ${ }^{40}$ The PEG/PLLA multiblock copolymers synthesized by the group of Jeong, having a total $\mathrm{M}_{\mathrm{n}}$ of $7 \mathrm{~kg} / \mathrm{mol}$, exhibited a CGC of approximately $30 \mathrm{w} / \mathrm{v} \%$ and underwent a sol-gel-sol transition with increasing temperature. ${ }^{38}$ The gelation mechanism was considered to be due to micellar aggregation. The transition temperature and gel modulus could be controlled by varying the PLLA block length and the PEG molecular weight. The in situ gel forming ability of the polymers was demonstrated by subcutaneous injection into rats. The PEG/PLLA multiblock copolymer showed a lower CGC and improved mechanical properties in comparison with an analogous PEG/PDLLA multiblock copolymer, ${ }^{39}$ which was attributed to a lower dynamic molecular motion and a higher aggregation tendency of PLLA due to the isotactic localization of the hydrophobic methyl groups.

Next to the linear PEG-PLA copolymers, also a number of star shaped and branched architectures have been explored for the preparation of thermo-responsive hydrogels. Park et al. synthesized 3armed PLA centered star block copolymers by coupling monocarboxylated PEG to a 3-armed hydroxyl terminated PLA in the presence of dicyclohexylcarbodiimide (DCC) as coupling agent. ${ }^{41}$ At a similar PEG block length of $5 \mathrm{~kg} / \mathrm{mol}$, an increase in the PLA blocks length led to an expanded gelation window. The 3 -armed star block copolymer exhibited a lower CGC in comparison with a 
PEG-PLA-PEG triblock copolymer possessing the same PEG content. 8-Armed PEG-PLA star block copolymers prepared by ring opening polymerization of L-lactide using 8-armed PEG with hydroxyl end functional groups as an initiator affords a star shaped BAB type copolymer. ${ }^{34}$ These star block copolymers, with a PEG content of $74 \mathrm{wt} \%$, exhibited approximately the same gelation behavior as PLLA-PEG-PLLA triblock copolymers with a PEG content of 84 wt \%. Importantly, the CGC at room temperature decreased from 40 to $15 \mathrm{w} / \mathrm{v} \%$ when the PLLA block length was increased from 10 to 14 lactyl units. Increasing the PEG molecular weight at a constant PLLA block length also resulted in a lower CGC possibly due to enhanced chain entanglements. Recently, highly branched PEG-PLLA copolymers were synthesized by a coupling reaction of 8-armed amine functionalized PEG and macromonomers having 2 PLLA arms and a N-hydroxysuccinimide activated ester group at the center of the polymer chain (Figure 4). ${ }^{42}$
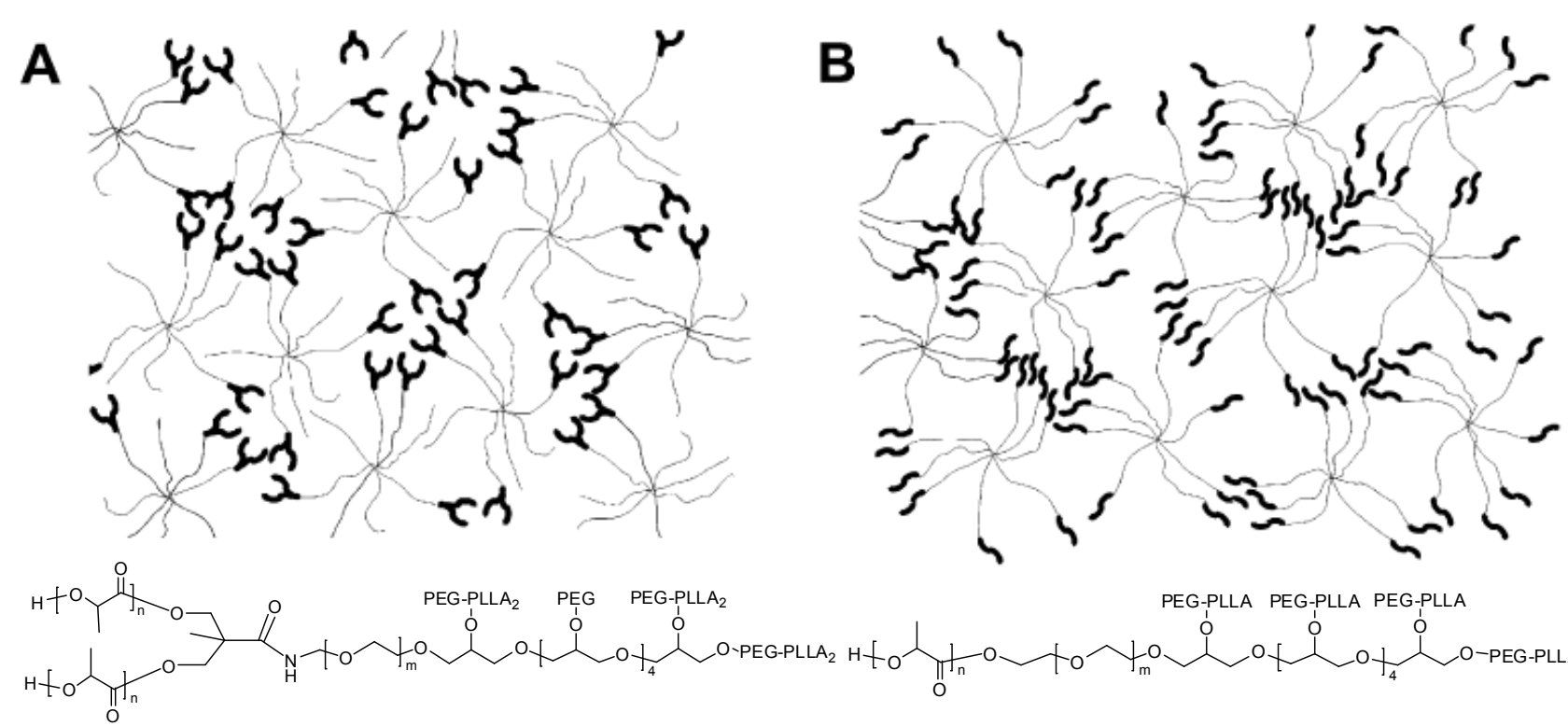

PEG-PLLA PEG-PLLA PEG-PLLA

O

Figure 4. Representation of a hydrogel prepared with the highly branched PEG-PLA block copolymer described by Velthoen et al. $(\mathrm{A})^{42}$ and the 8 -armed PEG-PLA star block copolymer described by Hiemstra et al. (B). ${ }^{43}$ Reprinted from reference 42 . Copyright 2011, with permission from Elsevier.

It was reported that 4 out of 8 PEG arms were functionalized with a branched PLA moiety. The copolymers showed a thermo-responsive gelation behavior at low concentrations ( $4 \mathrm{w} / \mathrm{v} \%)$. The gel-sol transition temperature could be tuned by varying the copolymer concentration and the molecular weight of the PLLA block. Branched block copolymers with a PLLA block length of 12 lactyl units exhibited significantly lower CGCs compared to the 8-armed PEG-PLLA star block copolymers with a similar PEG content and a PLLA block length of 10 lactyl units. ${ }^{34}$ This was ascribed to stronger hydrophobic interactions in the branched system, because hydrophobic 
domains may be formed more easily if only 4 out of 8 arms have to be folded into such a domain instead of 8 out of 8 arms (Figure 4).

Stereocomplexed hydrogels. A polymer stereocomplex is defined as a stereoselective interaction between two complementing stereoregular polymers, which interlock and form a new composite with altered physical properties in comparison with the constituting polymers. ${ }^{44}$ The complementary enantiomeric polymers PLLA and PDLA are optically active polymers with identical chemical structures but opposite configuration. PLLA forms a left-handed helix, while PDLA forms a right-handed helix. It has been suggested that the Van der Waals forces between the two helices are the driving force for a dense packing of the helices in a stereocomplex (Figure 5). ${ }^{45}$ Recently, stereocomplexation between enantiomeric PLLA and PDLA blocks in amphiphilic copolymers has been employed for the preparation of injectable hydrogels. Because stereocomplex crystals are formed at shorter PLA block lengths compared to homopolymer crystals, an operation window exists in which mixing of aqueous solutions of PLLA and PDLA block copolymers results in the formation of a hydrogel through crosslinking by stereocomplexation.

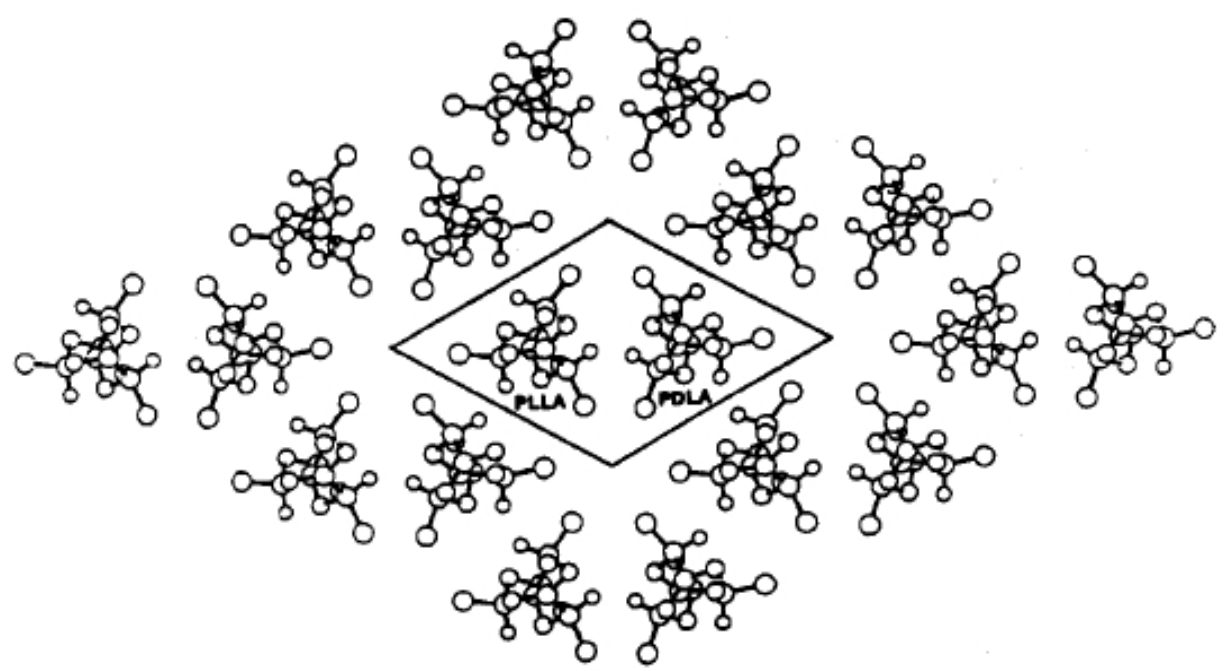

Figure 5. PLLA and PDLA molecular arrangements in a stereocomplex crystal. ${ }^{46}$ Reprinted with permission from John Wiley \& Sons, Inc.

Kimura and coworkers investigated the influence of the architecture of stereocomplexed PEGPLA block copolymers on the gelation properties. ${ }^{47,48}$ Ring opening polymerization of L- or Dlactide initiated by mono- or dihydroxyl PEG generated enantiomeric AB diblock and BAB triblock copolymers, respectively, whereas ABA triblock copolymers were obtained by coupling the $\mathrm{AB}$ diblock copolymers with HMDI. The PEG content of all copolymers in these studies was approximately $50 \mathrm{wt} \%$. Whereas BAB type copolymers may show thermo-reversible gelation, mixing of aqueous solutions of enantiomeric BAB block copolymers afforded systems that exhibit 
an irreversible sol-gel transition upon temperature increase. The initially present PLLA-PEG-PLLA and PDLA-PEG-PDLA type micelles consist of a core region and a PEG shell (Figure 6). When heated, the aggregation of the PLLA and PDLA segments at the core/shell interface of the micelles is weakened to allow the PLLA and PDLA polymer blocks (or segments thereof) to diffuse outside of the core and interact. Consequently, stereocomplexation is facilitated and the micelles become crosslinked and a gel is formed. Due to the high stability of the stereocomplex crystals, the gel formation is irreversible and no gel-sol transitions do occur upon cooling or heating.

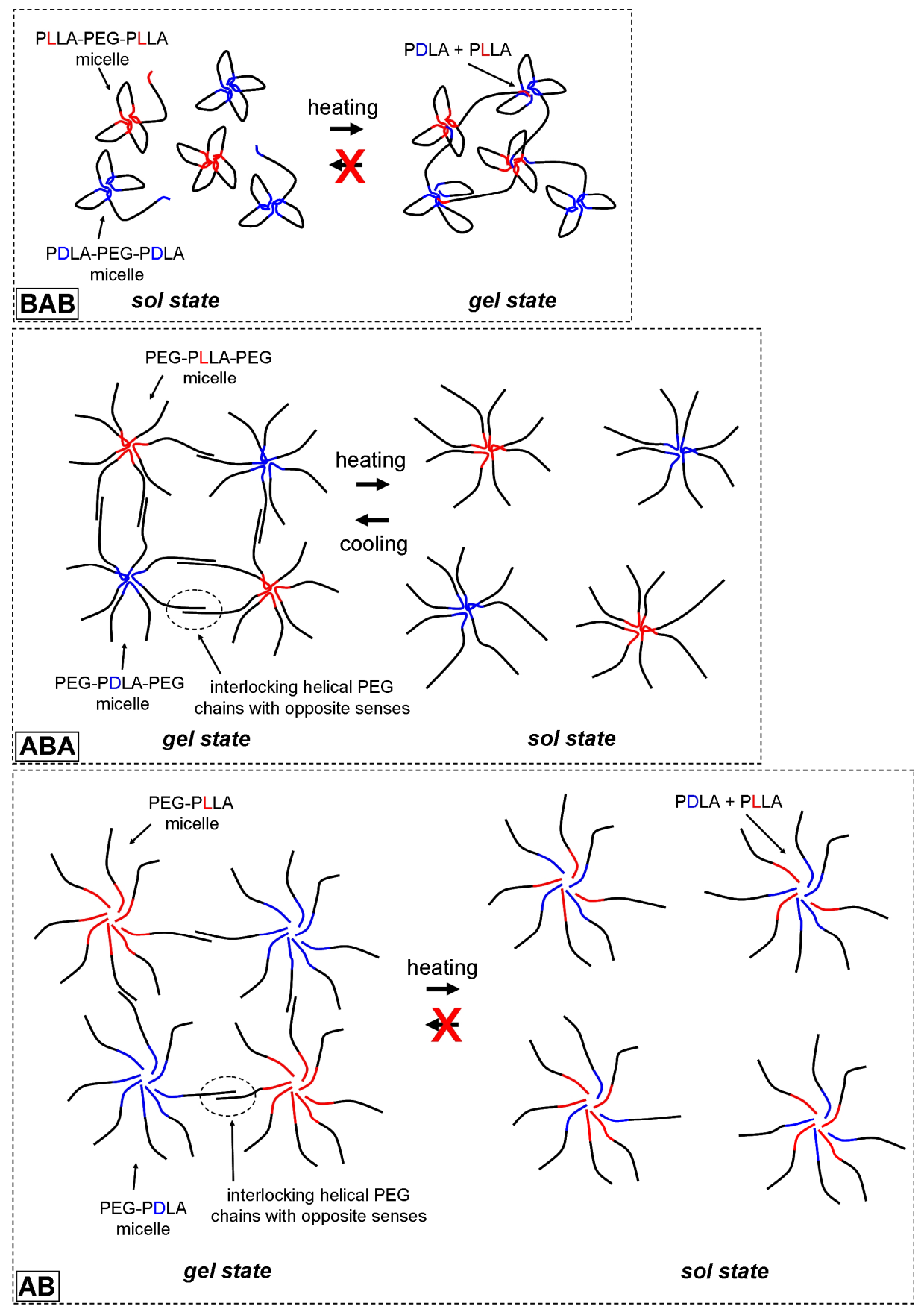

Figure 6. Proposed gelation mechanisms of enantiomeric mixtures of $\mathrm{BAB}, \mathrm{ABA}$ or $\mathrm{AB}$ type block copolymers, with A as the hydrophilic PEG block and B as the hydrophobic PLA block. ${ }^{49}$ 
Triblock copolymers with an ABA structure, on the other hand, formed hydrogels at high concentrations showing a reversible gel-sol transition with temperature (Figure 6). In aqueous solutions, the central PLLA and PDLA blocks are not easily exchanged among the micelles even when heated to high temperatures. It is suggested that the helical conformation of the PLLA and PDLA blocks is transmitted to the PEG chains and that aggregation of helical PEG chains with opposite senses leads to gelation at low temperatures. When the temperature is increased, the PEG domains collapse due to dehydration and the gel transforms into a sol. Upon cooling the intermicellar PEG crosslinks are able to form again and the system returns to the gel state. Mixed solutions of enantiomeric AB diblock copolymers also yielded hydrogels that exhibit a gel-sol transition upon temperature increase. However, unlike the ABA triblock system, this transition is irreversible (Figure 6). The authors suggested that the exchange of the core PLLA or PDLA blocks between micelles is much faster than in the ABA system, which was supported by wide angle X-ray scattering (WAXS) measurements showing that the stereocomplex crystals grow with increasing temperature. Eventually most micelles comprise stereocomplexed PDLA and PLLA blocks in their core at elevated temperatures. Intermicellar PEG interactions, which are responsible for the gelation of the system at lower temperatures, are weakened. The change in PEG interactions from inter- to intramicellar is the reason for the irreversibility of the gel-sol transition in the AB system.

Li et al. reported on the synthesis, characterization and stereocomplex mediated gelation of PEGPLA diblock and PLA-PEG-PLA (BAB) triblock copolymers. ${ }^{50-53}$ In a recent paper, they also investigated the effect of the PLA block length on the gelation behavior of stereocomplexed PLAPEG-PLA triblock copolymers. ${ }^{54}$ When the copolymers were synthesized by ring opening polymerization of L- or D-lactide initiated by dihydroxyl PEG $\left(\mathrm{M}_{\mathrm{n}} 4 \mathrm{~kg} / \mathrm{mol}\right)$ and zinc lactate as a catalyst for $7 \mathrm{~d}$, hydrogel formation by stereocomplexation was detected for copolymers with PLA blocks of 17 lactyl units but not for copolymers with PLA blocks of 11-13 lactyl units. This was ascribed to racemization of L-lactyl units leading to non-isotactic sequences in the PLLA chains, which prevents the formation of stereocomplexes. Racemization was largely reduced when the reaction time was shortened to $1 \mathrm{~d}$. It appeared that 10 lactyl units per PLA block were sufficient for the formation of stereocomplexed PLA-PEG-PLA hydrogels.

Stereocomplexing PEG-PDLA and PEG-PLLA $\left(\mathrm{AB}_{\mathrm{n}}\right)$ multiblock copolymers were prepared by coupling BAB triblock copolymers, having a $\mathrm{M}_{\mathrm{n}}$ of $12 \mathrm{~kg} / \mathrm{mol}$ and a PLA block length of 14 lactyl units, using diisocyanobutane. ${ }^{55}$ These stereocomplexed multiblock copolymers showed a lower CGC, faster gelation and a higher storage modulus in comparison with the parent triblock copolymers.

Star shaped block copolymers of PEG and PLA, showing stereocomplex mediated gelation, have also been investigated. It was found that stereocomplexed PEG-(PLA) 8 star block copolymers, prepared by ring opening polymerization of L- or D-lactide initiated by 8-armed PEG $\left(\mathrm{M}_{n} 20\right.$ 
$\mathrm{kg} / \mathrm{mol}$ ), gelate faster and form hydrogels with improved mechanical strength as compared to stereocomplexed PLA-PEG-PLA triblock copolymers. ${ }^{34}$ This was ascribed to a higher number of stereocomplex sites in PEG-(PLA) 8 . Rheological measurements showed that increasing the PLA block length from 12 to 14 lactyl units at a polymer concentration of $10 \mathrm{w} / \mathrm{v} \%$ resulted in an increase in the storage modulus from 0.9 to $7.0 \mathrm{kPa}$ and a decrease in gelation time from $40 \mathrm{~min}$ to less than $1 \mathrm{~min}$. The thermal reversibility of such a gel system was not reported.

Nagahama et al. prepared enantiomeric 8-armed PEG-PLA-PEG type copolymers (Figure 7) by coupling monocarboxylated PEG to star shaped PEG-PLLA or PEG-PDLA diblock copolymers using DCC as a coupling agent. ${ }^{56}$ At low concentrations an aqueous mixture consisting of both enantiomers yielded a sol at room temperature exhibiting an irreversible transition to the gel state upon temperature increase. It was suggested that partial dehydration of PEG blocks on heating leads to perturbation of the micellar core-shell structure, which accelerates stereocomplex formation to produce physical crosslinking of the micelles, resulting in a hydrogel. Similar to the linear BAB type triblock copolymers described by Kimura et al., ${ }^{47}$ the irreversibility of the hydrogel can be ascribed to the formation of a highly phase separated structure with high thermodynamic stability of stereocomplexed domains in micelles and/or aggregates. In vitro degradation experiments revealed a faster molecular weight reduction for copolymers in single enantiomer hydrogels compared to mixed enantiomer hydrogels. This suggests that stereocomplex formation has an inhibitory effect on the hydrolysis of the ester groups in the PLA domains.

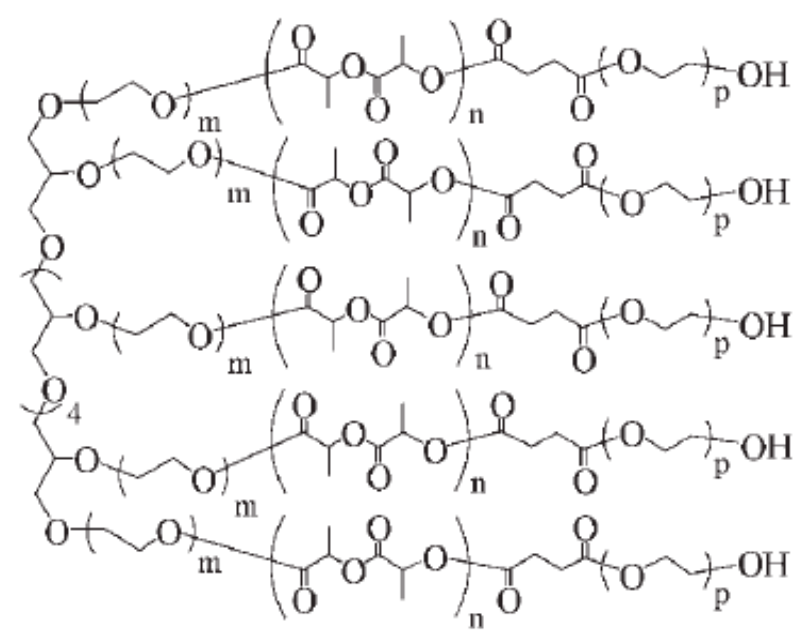

Figure 7. Structure of 8-armed PEG-PLA-PEG type copolymer used for the preparation of stereocomplexed hydrogels. ${ }^{56}$ Reprinted with permission from John Wiley \& Sons, Inc.

Hydrogels crosslinked by other physical interactions. Cyclodextrins (CDs) are cyclic oligosaccharides possessing a hydrophobic cavity that can act as a host to a variety of molecules. Aqueous solutions of CDs can form stable complexes with a range of polymers. This self-assembly phenomenon was used to create physically crosslinked hydrogels. ${ }^{57} \mathrm{~A}$ variety of biocompatible 
polymers, including Pluronics, ${ }^{58}$ reverse Pluronics (PPO-PEO-PPO) ${ }^{59}$ as well as linear ${ }^{60}$ and star shaped $\mathrm{PEG}^{61}$ in combination with CDs were studied. Disadvantages of these systems include low stability and long gelation times of several hours. Faster gelation was achieved when aqueous solutions of CDs were mixed with PCL-PEG-PCL. ${ }^{62}$ These systems formed hydrogels within minutes through a combination of inclusion complexation and micellar interactions.

Only a few reports on metallo-hydrogels, in which the reversible bonds between macromonomers are based on metal-ligand coordination, have been published. ${ }^{63-65}$ Pluronics and PEG polymers end functionalized with ligands such as 2,2':6',2' '-terpyridine (tpy) or 2,2'-bipyridine (bpy) yield hydrogels in aqueous solutions in the presence of transition metal ions including $\mathrm{Fe}(\mathrm{II})$ and $\mathrm{Ni}(\mathrm{II})$.

\subsection{Chemically crosslinked hydrogels}

Compared to physically crosslinked hydrogels, chemically crosslinked hydrogels usually have better mechanical properties and are more resistant to degradation. Disadvantages are health risks associated with reactive macromonomers and/or crosslinking agents, and practical limitations concerning reaction initiation. However, several chemical crosslinking methods were developed that proceed under mild reaction conditions, allowing for in situ hydrogel formation.

Photopolymerization. Although unsaturated groups can be polymerized using thermal or redox initiation, there are benefits to using photoinitiation for network formation. ${ }^{66}$ The primary advantage is the temporal and spatial control over the polymerization reaction, which allows control over polymerization exotherms and time of gelation. Moreover, photopolymerization can be used for the fabrication of complex structures via lasers or masks. ${ }^{67}$ Concerns exist about the limited penetration depth of light in thick constructs. The presence of harmful radicals generated during the polymerization process may damage cells or inactivate bioactive molecules. However, several groups reported the successful encapsulation of cells ${ }^{68,69}$ or proteins ${ }^{70,71}$ in photopolymerized constructs.

Photocrosslinked, biodegradable hydrogels prepared by UV irradiation of (meth)acrylate endcapped PEG-PLA block copolymers have been studied extensively. Pioneering work was performed by the group of Hubbell, who end functionalized PLA-PEG-PLA triblock copolymers with acrylate groups by reaction with acryloyl chloride in the presence of triethylamine. ${ }^{72}$ The resultant reactive macromonomers showed a fast gelation in water, despite the use of a relatively low initiator concentration, a low UV intensity and the presence of oxygen, which may act as a radical scavenger. The rapid gelation was ascribed to the formation of a micellar structure with a high concentration of double bonds within the hydrophobic domains, leading to a high propagation rate of the free radical polymerization. Differential scanning calorimetry (DSC) measurements showed that $80-90 \%$ of the total water content in the swollen networks corresponds to free water in 
the PEG domains. The remaining water was bound strongly with the PEG chains and comprised 2-3 water molecules per oxyethylene repeating unit. The degradation times of the hydrogels could be tuned from 0.3 to $120 \mathrm{~d}$ by altering the molecular weight and branching of the PEG and the length of the PLA block. Metters et al. investigated the degradation behavior of similar photocrosslinked PEG-PLA hydrogels in detail. ${ }^{73}$ They distinguished 3 phases during the degradation of the networks (Figure 8).

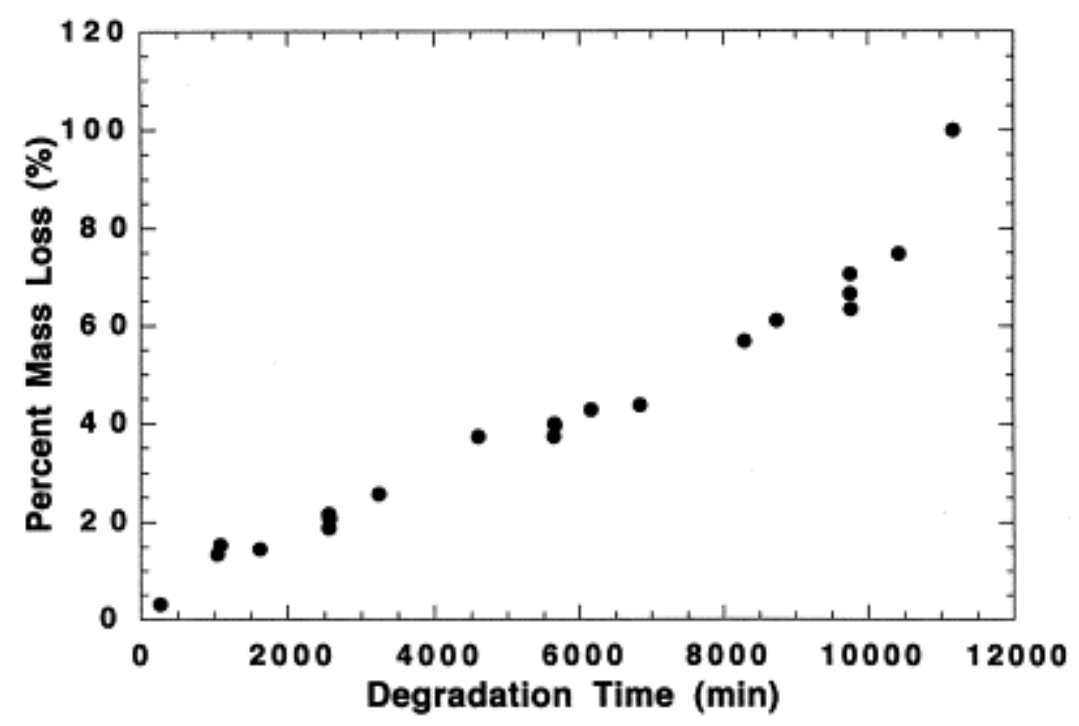

Figure 8. Mass loss for a hydrogel photopolymerized from a $50 \mathrm{w} / \mathrm{v} \%$ solution of an acrylated PLA-PEG-PLA (700-4600-700) triblock copolymer. Reprinted from reference 73. Copyright 2000, with permission from Elsevier.

The initial mass loss was caused by the degradation of the PLA blocks and their diffusion out of the gel. In the second phase the degradation rate of the ester linkages decreases with degradation time. However, the probability of the release of polyacrylate chains, initially held in place by numerous crosslinks, increases, resulting in a constant mass loss rate during the second phase. The final burst of mass loss corresponds to the sudden dissolution of remaining, uncrosslinked polymer chains. It was also shown that the volumetric swelling ratio of the networks exponentially increased and the compressive modulus exponentially decreased with degradation. Altering the crosslink density by changing the initial macromonomer concentration of the hydrogels modulated the timing of this behavior. The same group developed statistical kinetic models which were capable of accurately predicting the cleavage of crosslinks and hence the degradation behavior of photocrosslinked PEG-PLA networks. ${ }^{74,75}$

Lee et al. prepared photocrosslinked nanogels by UV irradiation of diacrylated PLA-PEG-PLA triblock copolymer micelles in dilute aqueous solution in the presence of ethylene glycol dimethacrylate as a crosslinker (Figure 9). ${ }^{76}$ It was shown that several physical properties of the nanogels, such as size, swelling behavior and drug release properties, could be manipulated by 
varying the concentration of ethylene glycol dimethacrylate. Photopolymerized hydrogels prepared from diacrylated PLA-PEG-PLA macromonomers have been applied in various biomedical applications, such as scaffolds for cartilage tissue engineering ${ }^{77}$ and systems for the controlled delivery of neural growth factors. ${ }^{78}$

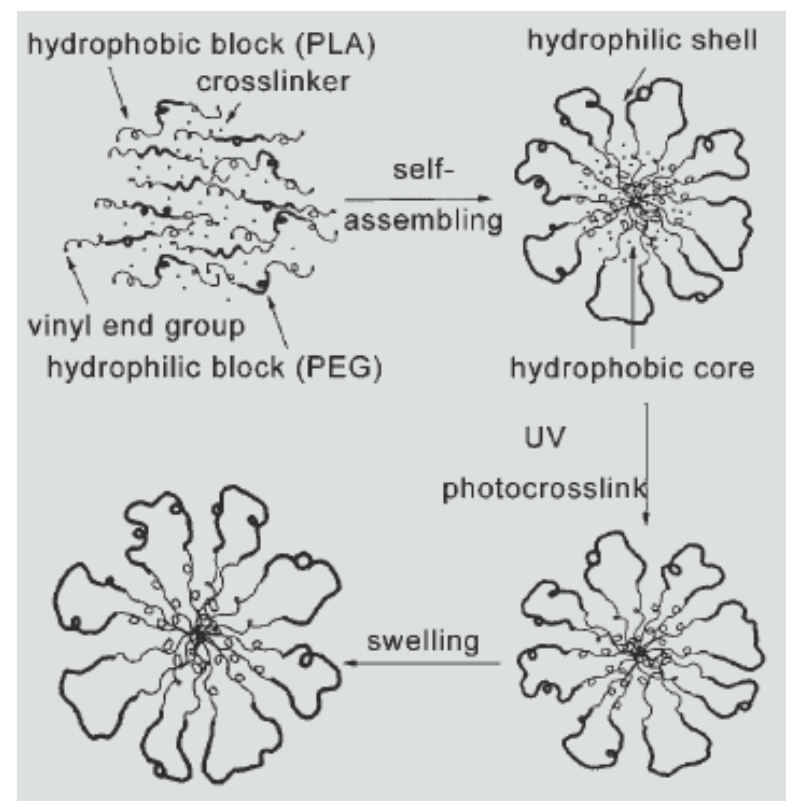

Figure 9. Formation of photocrosslinked PEG-PLA nanogels. ${ }^{76}$ Reprinted with permission from John Wiley \& Sons, Inc.

End group acrylated PEG-PLA block copolymers with an ABA structure have also been used for the synthesis of hydrogels. Clapper et al. photocrosslinked di(meth)acrylated PEG-PLA-PEG triblock copolymers of relatively low molecular weight (400-700-400) by UV irradiation. ${ }^{79}$ The acrylated macromonomers showed a significantly faster gelation than their methacrylated analogs, which was attributed to the steric hindrance of the methyl substituent in the methacrylate group. Apart from the gelation kinetics, the methacrylate and acrylate analogs displayed very similar properties. Networks prepared at a $75 \mathrm{w} / \mathrm{v} \%$ macromonomer concentration were stable for $160 \mathrm{~d}$ in vitro. This is significantly longer than photocrosslinked networks prepared from diacrylated PLAPEG-PLA (700-4600-700) at a similar concentration as described by Metters. ${ }^{73}$ The enhanced stability is possibly due to a higher crosslink density resulting from the low macromonomer molecular weight.

In a recent study, stereocomplexation and photocrosslinking were combined for the preparation of in situ forming, robust PEG-PLA networks. ${ }^{80}$ Two types of methacrylate functionalized 8-armed PEG-PLLA and PEG-PDLA star block copolymers were prepared, bearing the methacrylate groups either at the PLA chain ends (PEG-PLA-MA) or at the PEG chain ends (PEG-MA/PLA). It was shown that stereocomplexed hydrogels could be formed rapidly and that the methacrylate groups hardly interfered with the stereocomplexation. When the stereocomplexed hydrogels were 
subsequently photopolymerized, the storage moduli largely increased. The storage modulus of stereocomplexed and photopolymerized PEG-PLA-MA hydrogels was found to be highly dependent on the stereocomplex equilibration time before irradiation. This observation may be due to the slow formation of the stereocomplexes. In vitro degradation experiments showed that the stereocomplexed and photocrosslinked PEG-PLA-MA hydrogels completely dissolved after 3 weeks, whereas PEG-MA/PLA hydrogels prepared at similar macromonomer concentrations retained their integrity after 16 weeks. This was attributed to the slower hydrolysis of the ester bonds of the photopolymerized methacrylate groups compared to the ester bonds in the PLA chain.

Crosslinking by reaction between complementary groups. Several researchers prepared chemically crosslinked hydrogels by reaction of macromonomers endcapped with complementary reactive groups. Within this category, hydrogels synthesized by a Michael addition reaction between thiols and vinylic groups constitute an important class. This reaction proceeds at room temperature without catalysts or initiators, does not produce by-products, and is specific to thiols rather than amines. ${ }^{81}$ Moreover, thiols in peptides and other biomolecules can also be incorporated prior to gelation. The main disadvantages of this reaction are the relatively long polymerization times, the risk of denaturing thiol group bearing biomolecules upon in situ gelation, and the lack of spatial and temporal control over the network structure. ${ }^{82}$

Hubbell et al. reported on hydrogels prepared by Michael addition between multi-armed PEG acrylate and PEG dithiol or dithioerythritol. ${ }^{81,83}$ They showed that the hydrolysis of the acrylate ester bond increased by several orders of magnitude as a result of the proximity of the thioether group. ${ }^{84}$ The network degradation time could be tuned from 1 week to several months by altering the branching and molecular weight of the PEG acrylate. Several other research groups employed a Michael addition reaction between multifunctional PEG acrylates and thiol group containing crosslinkers for the preparation of degradable networks. ${ }^{85,86}$ It has been shown that at low PEG acrylate concentrations intramolecular reactions that lead to cyclization and network non-ideality are favored. ${ }^{84}$ To overcome these issues, Lutolf et al. pioneered the use of vinyl sulfone functionalized PEG for the preparation of networks by Michael addition. ${ }^{87}$ The high reactivity of the vinyl sulfone group facilitated incorporation of cysteine containing peptides comprising the cell adhesive RGD (arginine-glycine-aspartic acid) sequence, or sequences that can be cleaved by matrix metalloproteinases (MMPs) excreted by cells in the proximity of the construct. ${ }^{88}$ Networks containing covalently attached vascular endothelial growth factor (VEGF) were shown to stimulate angiogenesis in vivo (Figure 10). ${ }^{89}$ 


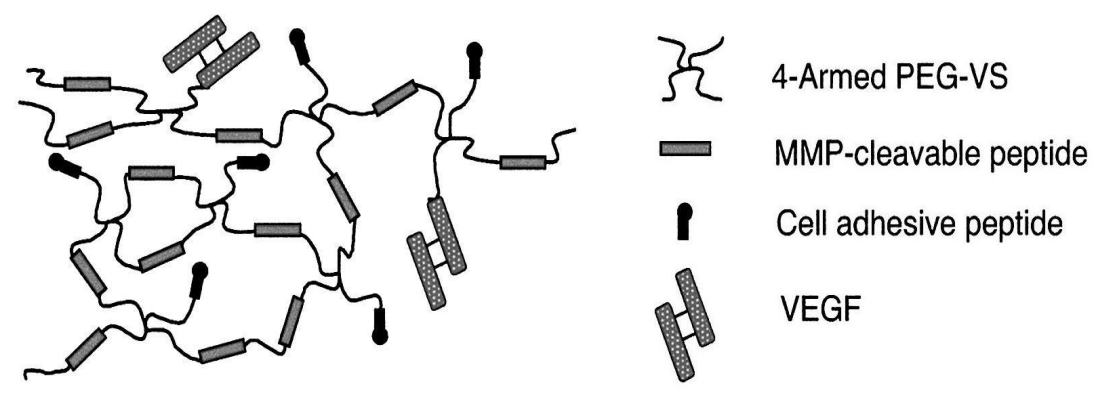

Figure 10. PEG vinylsulfone (PEG-VS) hydrogel with covalently incorporated bioactive moieties. ${ }^{89}$ Reprinted with permission from the Federation of American Societies for Experimental Biology.

Shikanov et al. reported that trifunctional thiol group containing peptides applied to 4-armed PEG vinyl sulfones decreased gelation times and hydrogel swelling relative to bifunctional crosslinkers. $^{90}$ In contrast to bifunctional peptides, the additional branching point prevents the formation of loops (intramolecular crosslinking). Zustiak et al. synthesized degradable networks by crosslinking PEG vinyl sulfone with PEG dithiol containing a labile ester group. ${ }^{91}$ The degradation time could be extended from several hours to several days by increasing the crosslinker molecular weight and the total polymer concentration. They also showed that the degradation rate significantly decreased when the number of methylene units between the ester and the thiol moieties of the crosslinker increased, which was ascribed to a more hydrophobic environment for the ester group. Associated physical properties changed predictably with degradation, as the storage modulus decreased whereas the swelling and the mesh size increased until the gels reached complete degradation.

Various other combinations of complementary reactive groups have been used for the preparation of PEG hydrogels, including amine groups in combination with (activated) ester groups ${ }^{92,93}$ and azide groups in combination with alkyne groups (also known as click chemistry). ${ }^{94,95}$

\subsection{Conclusions}

Over the past few decades, PEG based hydrogels have been applied in numerous biomedical applications resulting from their excellent biocompatibility. Hydrogels based on block copolymers of PEG and aliphatic polyesters are of particular interest because they can be degraded in the body by hydrolysis, thus eliminating the need for explantation after their function. Within this category, PEG-PLA block copolymers have been widely investigated. Several research groups devoted much effort to hydrogels that can be formed in situ under physiological conditions because of the significant advantages compared to gel constructs pre-made before implantation, such as ease of application, proper shape adaptation and the facile incorporation of biological components. Physical crosslinks, such as hydrophobic interactions and stereocomplexation, can be formed under mild 
conditions, but the resulting hydrogels often suffer from weak mechanical properties. Chemical crosslinking in general provides mechanically stable gels, but potential drawbacks include the toxicity of reactive macromonomers and crosslink agents as well as practical limitations concerning reaction initiation. Recent advances in photopolymerization technology allow for the preparation of complex structures in which the presentation of bioactive moieties can be spatially controlled. Alternatively, a Michael addition reaction between thiol and acrylate groups has proven a particularly suitable mechanism for in situ chemical crosslinking because it occurs at physiological conditions without the need for toxic initiators.

The examples discussed in this chapter represent some of the approaches that have been used to synthesize hydrogels for the treatment of biological and medical problems. The development of new materials and a deeper understanding of underlying gelation mechanisms will inevitably result in an even greater role for hydrogels in biomedical applications.

\subsection{References}

[1] Peppas, N. A.; Khare, A. R. Adv. Drug Delivery Rev. 1993, 11, 1-35.

[2] Wichterle, O.; Lim, D. Nature 1960, 185, 117-118.

[3] Lutolf, M. P.; Hubbell, J. A. Nat. Biotechnol. 2005, 23, 47-55.

[4] Tibbitt, M. W.; Anseth, K. S. Biotechnol. Bioeng. 2009, 103, 655-663.

[5] Khademhosseini, A.; Langer, R.; Borenstein, J.; Vacanti, J. P. Proc. Natl. Acad. Sci. U. S. A. 2006, 103, 2480-2487.

[6] Peppas, N. A.; Bures, P.; Leobandung, W.; Ichikawa, H. Eur. J. Pharm. Biopharm. 2000, 50, 27-46.

[7] Hennink, W. E.; van Nostrum, C. F. Adv. Drug Delivery Rev. 2002, 54, 13-36.

[8] Tessmar, J. K.; Gopferich, A. M. Adv. Drug Delivery Rev. 2007, 59, 274-291.

[9] Kroschwitz, J. I.; Mark, H. F. Encyclopedia of Polymer Science and Technology. 2003, Hoboken, NJ: Wiley-Interscience.

[10] Roorda, W. E.; Bodde, H. E.; Deboer, A. G.; Junginger, H. E. Pharm. Weekbl., Sci. Ed. 1986, 8, 165-189.

[11] Ruel-Gariepy, E.; Leroux, J. C. Eur. J. Pharm. Biopharm. 2004, 58, 409-426.

[12] Peretti, G. M.; Xu, J. W.; Bonassar, L. J.; Kirchhoff, C. H.; Yaremchuk, M. J.; Randolph, M. A. Tissue Eng. 2006, 12, 1151-1168.

[13] Wallace, D. G.; Rosenblatt, J. Adv. Drug Delivery Rev. 2003, 55, 1631-1649.

[14] Stevens, K. R.; Einerson, N. J.; Burmania, J. A.; Kao, W. Y. J. J. Biomater. Sci., Polym. Ed. 2002, 13, 1353-1366.

[15] Suh, J. K. F.; Matthew, H. W. T. Biomaterials 2000, 21, 2589-2598.

[16] Tonnesen, H. H.; Karlsen, J. Drug Dev. Ind. Pharm. 2002, 28, 621-630.

[17] Van Tomme, S. R.; Hennink, W. E. Expert Rev. Med. Devices 2007, 4, 147-164.

[18] Oh, E. J.; Park, K.; Kim, K. S.; Kim, J.; Yang, J. A.; Kong, J. H.; Lee, M. Y.; Hoffman, A. S.; Hahn, S. K. J. Controlled Release 2010, 141, 2-12.

[19] Jin, R.; Teixeira, L. S. M.; Krouwels, A.; Dijkstra, P. J.; van Blitterswijk, C. A.; Karperien, M.; Feijen, J. Acta Biomater. 2010, 6, 1968-1977.

[20] Lee, C. H.; Singla, A.; Lee, Y. Int. J. Pharm. 2001, 221, 1-22.

[21] Lin, C. C.; Anseth, K. S. Pharm. Res. 2009, 26, 631-643.

[22] Yamaoka, T.; Tabata, Y.; Ikada, Y. J. Pharm. Sci. 1994, 83, 601-606.

[23] Oh, J. K. Soft Matter 2011, 7, 5096-5108.

[24] Fusco, S.; Borzacchiello, A.; Netti, P. A. J. Bioact. Compat. Polym. 2006, 21, 149-164. 
[25] Zhong, Z. Y.; Dijkstra, P. J.; Feijen, J.; Kwon, Y. M.; Bae, Y. H.; Kim, S. W. Macromol. Chem. Phys. 2002, 203, 1797-1803.

[26] Yang, J.; Jia, L.; Hao, Q. H.; Li, Y.; Li, Q. B.; Fang, Q.; Cao, A. Macromol. Biosci. 2005, $5,896-903$.

[27] Wei, X. W.; Gong, C. Y.; Gou, M. Y.; Fu, S. Z.; Guo, Q. F.; Shi, S.; Luo, F.; Guo, G.; Qiu, L. Y.; Qian, Z. Y. Int. J. Pharm. 2009, 381, 1-18.

[28] Bat, E.; Feijen, J.; Grijpma, D. W. J. Controlled Release 2008, 132, e37-e39.

[29] Park, K.; Shalaby, W. S. W.; Park, H. Biodegradable hydrogels for drug delivery. 1993, Lancaster: Technomic.

[30] Alexandridis, P.; Hatton, T. A. Colloids Surf., A 1995, 96, 1-46.

[31] Jeong, B.; Kim, S. W.; Bae, Y. H. Adv. Drug Delivery Rev. 2002, 54, $37-51$.

[32] Jeong, B. M.; Lee, D. S.; Shon, J. I.; Bae, Y. H.; Kim, S. W. J. Polym. Sci., Part A: Polym. Chem. 1999, 37, 751-760.

[33] Choi, S. W.; Choi, S. Y.; Jeong, B.; Kim, S. W.; Lee, D. S. J. Polym. Sci., Part A: Polym. Chem. 1999, 37, 2207-2218.

[34] Hiemstra, C.; Zhong, Z. Y.; Dijkstra, P.; Feijen, J. Macromol. Symp. 2005, 224, 119-131.

[35] Li, S. M.; Molina, I.; Martinez, M. B.; Vert, M. J. Mater. Sci.: Mater. Med. 2002, 13, 8186.

[36] Agrawal, S. K.; Sanabria-DeLong, N.; Tew, G. N.; Bhatia, S. R. J. Mater. Res. 2006, 21, 2118-2125.

[37] Agrawal, S. K.; Sanabria-DeLong, N.; Tew, G. N.; Bhatia, S. R. Macromolecules 2008, 41, 1774-1784.

[38] Lee, J.; Bae, Y. H.; Sohn, Y. S.; Jeong, B. Biomacromolecules 2006, 7, 1729-1734.

[39] Joo, M. K.; Sohn, Y. S.; Jeong, B. Macromolecules 2007, 40, 5111-5115.

[40] Li, F.; Li, S. M.; Vert, M. Macromol. Biosci. 2005, 5, 1125-1131.

[41] Park, S. Y.; Han, B. R.; Na, K. M.; Han, D. K.; Kim, S. C. Macromolecules 2003, 36, 4115-4124.

[42] Velthoen, I. W.; van Beek, J.; Dijkstra, P. J.; Feijen, J. React. Funct. Polym. 2011, 71, 245-253.

[43] Hiemstra, C.; Zhong, Z.; Li, L. B.; Dijkstra, P. J.; Feijen, J. Biomacromolecules 2006, 7, 2790-2795.

[44] Slager, J.; Domb, A. J. Adv. Drug Delivery Rev. 2003, 55, 549-583.

[45] Tsuji, H. Macromol. Biosci. 2005, 5, 569-597.

[46] Ikada, Y.; Tsuji, H. Macromol. Rapid Commun. 2000, 21, 117-132.

[47] Fujiwara, T.; Mukose, T.; Yamaoka, T.; Yamane, H.; Sakurai, S.; Kimura, Y. Macromol. Biosci. 2001, 1, 204-208.

[48] Mukose, T.; Fujiwara, T.; Nakano, J.; Taniguchi, I.; Miyamoto, M.; Kimura, Y.; Teraoka, I.; Lee, C. W. Macromol. Biosci. 2004, 4, 361-367.

[49] Ottenbrite, R. M.; Park, K.; Okano, T. Biomedical Applications of Hydrogels Handbook. 2010, New York: Springer.

[50] Li, S. M.; Vert, M. Macromolecules 2003, 36, 8008-8014.

[51] Nouailhas, H.; El Ghzaoui, A.; Li, S.; Coudane, J. J. Appl. Polym. Sci. 2011, 122, 15991606.

[52] Zhang, Y.; Wu, X. H.; Han, Y. R.; Mo, F.; Duan, Y. R.; Li, S. M. Int. J. Pharm. 2010, $386,15-22$.

[53] Li, S. M.; El Ghzaoui, A.; Dewinck, E. Macromol. Symp. 2005, 222, 23-35.

[54] Nouailhas, H.; Li, F.; El Ghzaoui, A.; Li, S. M.; Coudane, J. Polym. Int. 2010, 59, 10771083.

[55] Hiemstra, C.; Zhong, Z.; Jiang, X.; Hennink, W. E.; Dijkstra, P. J.; Feijen, J. J. Controlled Release 2006, 116, e17-e19.

[56] Nagahama, K.; Fujiura, K.; Enami, S.; Ouchi, T.; Ohya, Y. J. Polym. Sci., Part A: Polym. Chem. 2008, 46, 6317-6332.

[57] Araki, J.; Ito, K. Soft Matter 2007, 3, 1456-1473.

[58] Li, J.; Li, X.; Zhou, Z. H.; Ni, X. P.; Leong, K. W. Macromolecules 2001, 34, 7236-7237. 
[59] Kuo, W. Y.; Lai, H. M. Polymer 2011, 52, 3389-3395.

[60] Watanabe, J.; Ooya, T.; Park, K. D.; Kim, Y. H.; Yui, N. J. Biomater. Sci., Polym. Ed. 2000, 11, 1333-1345.

[61] Sabadini, E.; Cosgrove, T. Langmuir 2003, 19, 9680-9683.

[62] Zhao, S. P.; Zhang, L. M.; Ma, D. J. Phys. Chem. B 2006, 110, 12225-12229.

[63] Vermonden, T.; de Vos, W. M.; Marcelis, A. T. M.; Sudholter, E. J. R. Eur. J. Inorg. Chem. 2004, 2847-2852.

[64] Fiore, G. L.; Klinkenberg, J. L.; Pfister, A.; Fraser, C. L. Biomacromolecules 2009, 10, 128-133.

[65] Chiper, M.; Hoeppener, S.; Schubert, U. S.; Fustin, C. A.; Gohy, J. F. Macromol. Chem. Phys. 2010, 211, 2323-2330.

[66] Anseth, K. S.; Burdick, J. A. Mrs Bull. 2002, 27, 130-136.

[67] Ifkovits, J. L.; Burdick, J. A. Tissue Eng. 2007, 13, 2369-2385.

[68] Hwang, N. S.; Varghese, S.; Theprungsirikul, P.; Canver, A.; Elisseeff, J. Biomaterials 2006, 27, 6015-6023.

[69] Burdick, J. A.; Anseth, K. S. Biomaterials 2002, 23, 4315-4323.

[70] Censi, R.; Vermonden, T.; Deschout, H.; Braeckmans, K.; di Martino, P.; de Smed, S. C.; van Nostrum, C. F.; Hennink, W. E. Biomacromolecules 2010, 11, 2143-2151.

[71] Burdick, J. A.; Mason, M. N.; Hinman, A. D.; Thorne, K.; Anseth, K. S. J. Controlled Release 2002, 83, 53-63.

[72] Sawhney, A. S.; Pathak, C. P.; Hubbell, J. A. Macromolecules 1993, 26, 581-587.

[73] Metters, A. T.; Anseth, K. S.; Bowman, C. N. Polymer 2000, 41, 3993-4004.

[74] Metters, A. T.; Bowman, C. N.; Anseth, K. S. J. Phys. Chem. B 2000, 104, 7043-7049.

[75] Metters, A. T.; Anseth, K. S.; Bowman, C. N. J. Phys. Chem. B 2001, 105, 8069-8076.

[76] Lee, W. C.; Li, Y. C.; Chu, I. M. Macromol. Biosci. 2006, 6, 846-854.

[77] Bryant, S. J.; Bender, R. J.; Durand, K. L.; Anseth, K. S. Biotechnol. Bioeng. 2004, 86, 747-755.

[78] Piantino, J.; Burdick, J. A.; Goldberg, D.; Langer, R.; Benowitz, L. I. Exp. Neurol. 2006, 201, 359-367.

[79] Clapper, J. D.; Skeie, J. M.; Mullins, R. F.; Guymon, C. A. Polymer 2007, 48, 6554-6564.

[80] Hiemstra, C.; Zhou, W.; Zhong, Z. Y.; Wouters, M.; Feijen, J. JACS 2007, 129, 99189926.

[81] Elbert, D. L.; Pratt, A. B.; Lutolf, M. P.; Halstenberg, S.; Hubbell, J. A. J. Controlled Release 2001, 76, 11-25.

[82] Liu, S. Q.; Tay, R.; Khan, M.; Ee, P. L. R.; Hedrick, J. L.; Yang, Y. Y. Soft Matter 2010, $6,67-81$.

[83] Van de Wetering, P.; Metters, A. T.; Schoenmakers, R. G.; Hubbell, J. A. J. Controlled Release 2005, 102, 619-627.

[84] Metters, A.; Hubbell, J. Biomacromolecules 2005, 6, 290-301.

[85] Pritchard, C. D.; O'Shea, T. M.; Siegwart, D. J.; Calo, E.; Anderson, D. G.; Reynolds, F. M.; Thomas, J. A.; Slotkin, J. R.; Woodard, E. J.; Langer, R. Biomaterials 2011, 32, 587 597.

[86] Yu, H. Q.; Cong, R. Chem. Pap. 2010, 64, 619-624.

[87] Lutolf, M. P.; Hubbell, J. A. Biomacromolecules 2003, 4, 713-722.

[88] Lutolf, M. P.; Raeber, G. P.; Zisch, A. H.; Tirelli, N.; Hubbell, J. A. Adv. Mater. 2003, $15,888-892$.

[89] Zisch, A. H.; Lutolf, M. P.; Ehrbar, M.; Raeber, G. P.; Rizzi, S. C.; Davies, N.; Schmokel, H.; Bezuidenhout, D.; Djonov, V.; Zilla, P.; Hubbell, J. A. FASEB J. 2003, 17, 2260-2285.

[90] Shikanov, A.; Smith, R. M.; Xu, M.; Woodruff, T. K.; Shea, L. D. Biomaterials 2011, 32, 2524-2531.

[91] Zustiak, S. P.; Leach, J. B. Biomacromolecules 2010, 11, 1348-1357.

[92] Brandl, F.; Kastner, F.; Gschwind, R. M.; Blunk, T.; Tessmar, J.; Gopferich, A. J. Controlled Release 2010, 142, 221-228. 
[93] Li, X.; Tsutsui, Y.; Matsunaga, T.; Shibayama, M.; Chung, U.; Sakai, T. Macromolecules 2011, 44, 3567-3571.

[94] Malkoch, M.; Vestberg, R.; Gupta, N.; Mespouille, L.; Dubois, P.; Mason, A. F.; Hedrick, J. L.; Liao, Q.; Frank, C. W.; Kingsbury, K.; Hawker, C. J. Chem. Commun. 2006, 2774-2776.

[95] Van Dijk, M.; van Nostrum, C. F.; Hennink, W. E.; Rijkers, D. T. S.; Liskamp, R. M. J. Biomacromolecules 2010, 11, 1608-1614. 


\title{
Chapter 3
}

\section{The influence of amide versus ester linkages on the properties of 8- armed PEG-PLA star block copolymer hydrogels}

This chapter has been published: Sytze J. Buwalda, ${ }^{a}$ Pieter J. Dijkstra, ${ }^{a}$ Lucia Calucci, ${ }^{b}$ Claudia Forte, $^{\mathrm{b}}$ and Jan Feijen ${ }^{\mathrm{a}}$ Biomacromolecules 2010, 11, 224-232.

${ }^{a}$ Department of Polymer Chemistry and Biomaterials, Faculty of Science and Technology, MIRA Institute for Biomedical Technology and Technical Medicine, University of Twente, P.O. Box 217, 7500 AE Enschede, The Netherlands

${ }^{\mathrm{b}}$ Istituto di Chimica dei Composti OrganoMetallici, CNR-Consiglio Nazionale delle Ricerche, Area della Ricerca di Pisa, via G. Moruzzi 1, 56124 Pisa, Italy

\begin{abstract}
Water soluble 8-armed poly(ethylene glycol)-poly(L-lactide) star block copolymers linked by an amide or ester group between the PEG core and the PLA blocks (PEG-(NHCO)-(PLA) 8 and PEG(OCO)-(PLA $)_{8}$ ) were synthesized by the stannous octoate catalyzed ring opening polymerization of L-lactide using an amine- or hydroxyl terminated 8-armed star PEG. At concentrations above the critical gel concentration, thermo-sensitive hydrogels were obtained, showing a reversible single gel to sol transition. At similar composition PEG-(NHCO)-(PLA) 8 hydrogels were formed at significantly lower polymer concentrations and had higher storage moduli. Whereas the hydrolytic degradation/dissolution of the PEG-(OCO)-(PLA) 8 takes place by preferential hydrolysis of the ester bond between the PEG and PLA block, the PEG-(NHCO)-(PLA) 8 hydrogels degrade through hydrolysis of ester bonds in the PLA main chain. Because of their relatively good mechanical properties and slow degradation in vitro, $\mathrm{PEG}-(\mathrm{NHCO})-(\mathrm{PLA})_{8}$ hydrogels are interesting materials for biomedical applications such as controlled drug delivery systems and matrices for tissue engineering.
\end{abstract}




\subsection{Introduction}

Hydrogels are polymer networks which are able to retain large amounts of water in their swollen state. $^{1-3}$ They generally exhibit excellent biocompatibility and are accordingly of interest for biomedical applications such as tissue engineering and systems for controlled delivery of biologically active agents. Several types of physical and chemical hydrogels are responsive to external stimuli like temperature and $\mathrm{pH}^{4}$ Among the thermo-responsive hydrogels, poly(ethylene oxide)-poly(propylene oxide)-poly(ethylene oxide) (PEO-PPO-PEO) triblock copolymers, commercially known as Pluronics or Poloxamers, have been widely investigated. At room temperature, a $20 \mathrm{w} / \mathrm{v} \%$ Pluronic F127 solution behaves as a viscous liquid, which is transformed into a semi-solid gel at body temperature. ${ }^{5}$ Gel formation is believed to occur as a result of dehydration of the PEG chains at elevated temperatures. A significant drawback of Pluronic hydrogels is their low mechanical strength, which originates from the weak hydrophobic interactions between PPO blocks. ${ }^{6}$ Furthermore, the absence of biodegradable groups in the backbone severely limits the use of relatively high molecular weight Pluronics in biomedical applications because of the limits to renal excretion. ${ }^{5}$ These drawbacks prompted several researchers to develop block copolymers of PEG and biodegradable polyesters as a basis for thermo-sensitive hydrogels. Kim and coworkers synthesized ABA triblock copolymers, with A as a hydrophilic PEG block and B as a hydrophobic poly(lactic acid) (PLA) block. ${ }^{7,8}$ Aqueous solutions of these copolymers form micelles at low concentrations and a gel at higher concentrations and temperatures, which is ascribed to the association of micelles. The gels transform into sols when the temperature is further increased, which was attributed to PEG corona shrinkage and accompanying micelle collapse, or to the change in micellar structure from a spherical to a cylindrical shape. Upon an increase of the PLA molecular weight from 2000 to $5000 \mathrm{~g} / \mathrm{mol}$, the critical gel concentration (CGC) at room temperature decreases as a result of increased hydrophobic interactions. Block copolymers with an inverted structure $(\mathrm{BAB})$, with $\mathrm{A}$ as the hydrophilic block, are generally prepared by ring opening polymerization of lactide initiated by the hydroxyl groups of PEG. ${ }^{9}$ At room temperature the CGC of for example triblock copolymers with a PEG $\mathrm{M}_{\mathrm{n}}$ of $12500 \mathrm{~g} / \mathrm{mol}$ drastically decreases from 80 to $10 \mathrm{w} / \mathrm{v} \%$ upon an increase of the PLA block length from 10 to 19 lactyl units, indicating a much stronger effect of the hydrophobic chain length on the gelation behavior in comparison with ABA type triblock copolymers. ${ }^{7,8}$ Bae et al. reported on the gelation behavior of aqueous solutions of ABA- and BAB type triblock copolymers of PEG and poly(Ecaprolactone) (PCL). ${ }^{10}$ Compared to PEG-PCL-PEG triblock copolymers possessing similar hydrophilic and hydrophobic block lengths, PCL-PEG-PCL polymers exhibited a larger gel window and a higher storage modulus. This was ascribed to the possibility of intermicellar PCL bridging for the BAB type triblock copolymers, leading to more facile micellar aggregation. Various other aliphatic polyesters have been used in linear block copolymers with PEG for the preparation of 
thermo-sensitive hydrogels, including poly(lactide-co-glycolide) (PLGA), ${ }^{11-13} \operatorname{poly}(\delta$ valerolactone $)^{14}$ and poly(3-methylglycolide). ${ }^{15}$

More recently, star shaped block copolymers of PEG and aliphatic polyesters, showing a thermosensitive gelation behavior, have been investigated. Lee et al. prepared 3- and 4-armed star block copolymers with PEG arms and a PLGA core. ${ }^{16}$ These star shaped block copolymers formed a gel at higher polymer concentrations than PEG-PLGA-PEG triblock copolymers with the same PEG block length and similar PLGA content due to hampered hydrophobic interactions and micellar aggregation. In a recent study, star shaped block copolymers with a PEG core and PLA arms were prepared by ring opening polymerization of lactide initiated by the hydroxyl groups of 8 -armed PEG. ${ }^{9,17}$ These 8-armed PEG-PLA star block copolymers showed a much lower CGC than linear PLA-PEG-PLA block copolymers with a similar PEG content due to the increased interactions between hydrophobic blocks. A gel to sol transition was found upon elevation of the temperature, and the transition temperature increased with increasing polymer concentration and hydrophobic chain length.

Physically crosslinked hydrogels are generally mechanically weak and appear to degrade rapidly or the network may be disrupted by local changes $(\mathrm{pH}$, ionic strength or temperature) in the environment. It has been suggested that preferential hydrolysis of the ester bond between the PEG and poly(lactide) may cause the rapid degradation of this type of block copolymers. ${ }^{18,19}$ This prompted us to investigate the properties and stability of PEG-poly(lactide) block copolymers linked through a hydrolytically stable amide bond. In this paper, we describe the synthesis and properties of 8-armed PEG-PLA star block copolymer gels, prepared from amine end functionalized 8-armed PEG. The physical, mechanical and degradation properties of hydrogels prepared from these block copolymers were compared to previously described block copolymers containing an ester linking unit.

\subsection{Experimental section}

Materials. Hydroxyl terminated 8-armed poly(ethylene glycol) (PEG-(OH) $8, \mathrm{M}_{\mathrm{n}}, \mathrm{NMR}=20600$ $\mathrm{g} / \mathrm{mol}$ and $23700 \mathrm{~g} / \mathrm{mol}$ ) was purchased from Jenkem (Allen, Texas, USA) and purified before use by dissolution in dichloromethane and precipitation in cold diethyl ether. L-lactide was obtained from Purac (Gorinchem, the Netherlands) and used as received. Tin(II) 2-ethylhexanoate (stannous octoate), methanesulfonyl chloride (mesyl chloride), triethylamine (TEA) and $25 \%$ aqueous ammonia solution were all from Sigma-Aldrich (St Louis, Missouri, USA). Dichloromethane, TEA and toluene were dried over calcium hydride, potassium hydroxide and sodium, respectively, and distilled prior to use. 
Synthesis. The 8-armed poly(ethylene glycol)-poly(L-lactide) star block copolymers (PEG(NHCO)-(PLA $\left.)_{8}\right)$ were synthesized by ring opening polymerization of L-lactide in toluene at 110 ${ }^{\circ} \mathrm{C}$. Amine terminated 8-armed star PEG $\left(\mathrm{PEG}-\left(\mathrm{NH}_{2}\right)_{8}\right)$ and stannous octoate were used as initiator and catalyst, respectively.

PEG-(NH$)_{8}$ was synthesized starting from PEG- $(\mathrm{OH})_{8}\left(\mathrm{M}_{\mathrm{n}, \mathrm{NMR}}=23700 \mathrm{~g} / \mathrm{mol}\right)$ following a twostep procedure analogous to that described by Elbert and Hubbell for linear hydroxyl terminated PEGs. ${ }^{20}$ In a typical procedure, PEG- $(\mathrm{OH})_{8}(30 \mathrm{~g}, 1.3 \mathrm{mmol})$ dissolved in $250 \mathrm{ml}$ of toluene was dried by azeotropic distillation of approximately $100 \mathrm{ml}$ of toluene in a nitrogen atmosphere. After cooling the solution with an ice bath, $100 \mathrm{ml}$ of dichloromethane was added. Subsequently, TEA (4.0 ml, $28.8 \mathrm{mmol})$ and mesyl chloride $(2.2 \mathrm{ml}, 28.8 \mathrm{mmol})$ were added dropwise under stirring. The reaction mixture was allowed to warm to room temperature and stirred overnight in a nitrogen atmosphere. The mixture was filtered, concentrated in vacuo and precipitated in a large excess of cold diethyl ether. The product was collected by filtration and dried in vacuo overnight. The PEG(mesylate) $)_{8}$ was added to $400 \mathrm{ml}$ of a $25 \%$ aqueous ammonia solution in a tightly closed bottle. The mixture was vigorously stirred for $4 \mathrm{~d}$ at room temperature, after which the ammonia was allowed to evaporate for $2 \mathrm{~d}$. The $\mathrm{pH}$ of the solution was raised to 13 with $60 \mathrm{ml}$ of a $1 \mathrm{M}$ sodium hydroxide solution, and the resulting solution was extracted 3 times with $100 \mathrm{ml}$ of dichloromethane. The combined extracts were dried with magnesium sulfate, concentrated in vacuo and precipitated in a large excess of cold diethyl ether. PEG-(NH$)_{8}$ was obtained by filtration and dried over phosphorus pentoxide. Conversion: $>97 \%$, yield: $89 \%$. ${ }^{1} \mathrm{H}$ NMR $\left(\mathrm{CDCl}_{3}\right): \delta=3.63(\mathrm{~m}, \mathrm{PEG}$ protons), 2.94 ( $\mathrm{t}, \mathrm{CH}_{2} \mathrm{CH}_{2} \mathrm{NH}_{2}$ ).

A typical procedure for the synthesis of a PEG-(NHCO)-(PLA) 8 star block copolymer with a degree of polymerization of the PLA arms of 13 was as follows. To a solution of PEG-( $\left.\mathrm{NH}_{2}\right)_{8}(5.00$ $\mathrm{g}, 0.2 \mathrm{mmol})$ in $22 \mathrm{ml}$ of dry toluene, L-lactide $(3.16 \mathrm{~g}, 21.9 \mathrm{mmol})$ and stannous octoate $(0.8 \mathrm{~g}, 2.0$ mmol) were added. The reaction was allowed to proceed for $4 \mathrm{~h}$ at $110^{\circ} \mathrm{C}$ in a nitrogen atmosphere. The mixture was filtered and concentrated under reduced pressure. The product was purified by precipitation in a 20-fold excess of a mixture of cold diethyl ether and methanol $(20 / 1 \mathrm{v} / \mathrm{v})$. PEG(NHCO)-( $\left.\mathrm{PLA}_{13}\right)_{8}$ was obtained by filtration and dried overnight in vacuo at room temperature over phosphorus pentoxide. ${ }^{1} \mathrm{H}$ NMR $\left(\mathrm{CDCl}_{3}\right): \delta=6.54\left(\mathrm{~s}, \mathrm{CH}_{2} \mathrm{NHCO}\right), 5.17\left(\mathrm{~m}, \mathrm{CH}_{3} \mathrm{CH}\right), 4.35(\mathrm{q}$, $\left.\mathrm{CH}_{3} \mathrm{CHOH}\right), 3.64$ (m, PEG protons), $2.68\left(\mathrm{~s}, \mathrm{CH}_{3} \mathrm{CHOH}\right), 1.57$ (m, $\left.\mathrm{CH}_{3} \mathrm{CH}\right), 1.48$ (d, $\left.\mathrm{CH}_{3} \mathrm{CHOH}\right)$.

The 8-armed PEG-(OCO)-(PLA $\left.{ }_{13}\right)_{8}$ star block copolymer was prepared similarly, using PEG$(\mathrm{OH})_{8}\left(\mathrm{M}_{\mathrm{n}, \mathrm{NMR}}=20600 \mathrm{~g} / \mathrm{mol}\right)$ as the initiator. ${ }^{1} \mathrm{H} \mathrm{NMR}\left(\mathrm{CDCl}_{3}\right): \delta=5.17\left(\mathrm{~m}, \mathrm{CH}_{3} \mathrm{CH}\right), 4.35(\mathrm{~m}$, $\left.\mathrm{CH}_{3} \mathrm{CHOH}\right), 4.29$ (m, $\left.\mathrm{CH}_{2} \mathrm{OCO}\right), 3.64$ (m, PEG protons), 2.69 (s, $\left.\mathrm{CH}_{3} \mathrm{CHOH}\right), 1.57$ (m, $\left.\mathrm{CH}_{3} \mathrm{CH}\right)$, $1.48\left(\mathrm{~d}, \mathrm{CH}_{3} \mathrm{CHOH}\right)$. 
Characterization. ${ }^{1} \mathrm{H}$ NMR $(300 \mathrm{MHz})$ spectra were recorded on a Varian Inova $300 \mathrm{NMR}$ spectrometer. Polymers were dissolved in $\mathrm{CDCl}_{3}$ at a concentration of $15 \mathrm{mg} / \mathrm{ml}$.

Intrinsic viscosities were determined by single point measurements $\left(\mathrm{CHCl}_{3}, \mathrm{C}=0.1 \mathrm{~g} / \mathrm{dl}, 25{ }^{\circ} \mathrm{C}\right)$ using an Ubbelohde $0 \mathrm{C}$ capillary viscometer. The following empirical relation was applied:

$$
\eta_{\text {intr }}=\sqrt{2} / \mathrm{C} \cdot \sqrt{\eta_{\mathrm{sp}}-\ln \eta_{\text {rel }}}
$$

Thermal properties of polymers were determined using DSC. Heating and cooling rates of 20 ${ }^{\circ} \mathrm{C} /$ min were applied. Samples were heated from 25 to $200{ }^{\circ} \mathrm{C}$, kept at $200{ }^{\circ} \mathrm{C}$ for $1 \mathrm{~min}$, cooled to $50{ }^{\circ} \mathrm{C}$, kept at $-50{ }^{\circ} \mathrm{C}$ for $1 \mathrm{~min}$, and finally heated to $200{ }^{\circ} \mathrm{C}$. Crystallization temperatures $\left(\mathrm{T}_{\mathrm{c}}\right)$ and corresponding enthalpies $\left(\Delta \mathrm{H}_{\mathrm{c}}\right)$ were obtained from the cooling scan, while melting temperatures $\left(\mathrm{T}_{\mathrm{m}}\right)$ and enthalpies $\left(\Delta \mathrm{H}_{\mathrm{m}}\right)$ were obtained from the second heating scan.

Aqueous solution properties. The critical association concentration (CAC) values of aqueous solutions of the PEG-PLA star block copolymers were determined using a dye solubilization method. $^{21}$ Aqueous solutions of PEG-(NHCO)-(PLA) 8 and PEG-(OCO)-(PLA 13$)_{8}$ star block copolymers in the concentration range of 0.001 to $5 \mathrm{w} / \mathrm{v} \%$ were prepared using distilled water. Repeated heating cycles were necessary to dissolve the polymer. A solution of the hydrophobic dye 1,6-diphenyl-1,3,5-hexatriene (DPH) was prepared in methanol at a concentration of $0.5 \mathrm{mM}$. Approximately $1 \mathrm{ml}$ of polymer solution was added to a polystyrene vial, followed by addition of $10 \mu \mathrm{l}$ of the DPH solution. The samples were allowed to equilibrate for at least $3 \mathrm{~h}$ in the dark, after which the absorption at $357 \mathrm{~nm}$ relative to a blank (polymer solution containing no DPH) was measured using a Varian Cary 300 Bio UV-visible spectrophotometer. The absorption was plotted against the polymer concentration and the intercept of the extrapolated straight lines was taken as the CAC.

Dynamic light scattering (DLS) of dilute solutions $(0.5 \mathrm{w} / \mathrm{v} \%)$ of PEG-PLA star block copolymers in water was performed to determine aggregate sizes. Experiments were carried out at 25 and $40{ }^{\circ} \mathrm{C}$ using a Malvern Nano ZS, a laser wavelength of $633 \mathrm{~nm}$ and a scattering angle of $173^{\circ}$. At each temperature, the sample was allowed to equilibrate for $10 \mathrm{~min}$.

Gel properties. The vial tilting method was used to determine the critical gel concentration (CGC) at room temperature as well as the temperature dependent gelation behavior of aqueous solutions of PEG-PLA star block copolymers. Polymers were dissolved in distilled water in tightly capped glass vials. The temperature dependent gelation behavior was studied in the range of 2 to 80 ${ }^{\circ} \mathrm{C}$ with temperature increments of $2{ }^{\circ} \mathrm{C}$. At each temperature, the sample was allowed to equilibrate for $10 \mathrm{~min}$. If there was no flow after tilting the vials $90^{\circ}$ for $1 \mathrm{~min}$, the sample was regarded as a gel; otherwise it was regarded as a sol. 
${ }^{1} \mathrm{H}$ NMR spectra were recorded on PEG-(NHCO)-(PLA $\left.)_{13}\right)_{8}(12 \mathrm{w} / \mathrm{v} \%)$ and PEG-(OCO)-(PLA 13$)_{8}$ $\left(22 \mathrm{w} / \mathrm{v} \%\right.$ ) hydrogel samples in $\mathrm{D}_{2} \mathrm{O}$. The samples were prepared directly in the NMR tube by adding $\mathrm{D}_{2} \mathrm{O}$ (99.98 \% Eurisotop) to the dry polymer. The ${ }^{1} \mathrm{H}$ NMR experiments were carried out on a Bruker AMX-300 WB spectrometer working at $300.13 \mathrm{MHz}$, using a $5 \mathrm{~mm}$ probe head, with a $90^{\circ}$ pulse of $6 \mu \mathrm{s}$, a recycle delay of $60 \mathrm{~s}$, and acquiring 8 scans. The spectra were recorded every 5 ${ }^{\circ} \mathrm{C}$ from $25{ }^{\circ} \mathrm{C}$ to $70{ }^{\circ} \mathrm{C}$; the temperature was controlled within $0.1{ }^{\circ} \mathrm{C}$. Relative peak intensities within each spectrum were determined by integration of the peaks obtained by spectral deconvolution using the SPORT-NMR software, ${ }^{22}$ and were then calibrated using a sample of 3(trimethylsilyl)-1-propanesulfonic acid sodium salt at known concentration in $\mathrm{D}_{2} \mathrm{O}$ as external standard. The same sample was also used as reference for the ${ }^{1} \mathrm{H}$ chemical shifts.

Oscillatory rheology experiments were performed to determine the mechanical properties of the hydrogels. The storage (G') and loss (G',) modulus of hydrogels were monitored for $30 \mathrm{~min}$ at 20 ${ }^{\circ} \mathrm{C}$ on an Anton-Paar Physica MCR 301 rheometer. The samples were heated to $70{ }^{\circ} \mathrm{C}$ at a heating rate of $1{ }^{\circ} \mathrm{C} / \mathrm{min}$ and cooled to $20{ }^{\circ} \mathrm{C}$ at the same rate. A layer of oil was applied on top of the gels to prevent water evaporation. Experiments were performed using a flat plate measuring geometry (diameter $25 \mathrm{~mm}$, gap $0.3 \mathrm{~mm}$ ) utilizing a strain of $0.5 \%$ and a frequency of $1 \mathrm{~Hz}$. To confirm that the strain is within the viscoelastic regime, an amplitude sweep from 0.1 to $10 \%$ was performed at $1 \mathrm{~Hz}$.

Degradation. Gravimetric degradation/dissolution experiments were performed to determine the stability of the hydrogels. On top of $0.5 \mathrm{ml}$ of hydrogel $3 \mathrm{ml}$ of PBS was placed and the samples were kept at $37{ }^{\circ} \mathrm{C}$. To prevent bacterial growth, $0.02 \mathrm{w} / \mathrm{v} \% \mathrm{NaN}_{3}$ was added to the buffer solution. At regular time intervals, $2.5 \mathrm{ml}$ of the supernatant was removed and replaced by fresh buffer. The supernatant samples, taken at the various time points, were freeze-dried and the mass of solubilized polymer was determined gravimetrically by subtracting the mass of the PBS salts from the mass of the sample. Degradation/dissolution experiments were performed twice. The structure of the remaining polymers in fully solubilized hydrogel samples was analyzed by ${ }^{1} \mathrm{H}$ NMR spectroscopy. After freeze-drying of a sample, polymers were dissolved in a minimal amount of dichloromethane. The solution was filtered and precipitated in an excess of a mixture of cold diethyl ether and methanol $(20 / 1 \mathrm{v} / \mathrm{v})$. The precipitate was collected by filtration, dried under a nitrogen flow and analyzed by ${ }^{1} \mathrm{H}$ NMR.

\subsection{Results and discussion}

Synthesis and characterization of PEG-PLA star block copolymers. PEG-(NHCO)-(PLA) 8 and PEG-(OCO)-(PLA) $)_{8}$ star block copolymers were prepared by ring opening polymerization of Llactide initiated by PEG-(NH$)_{8}$ and PEG- $(\mathrm{OH})_{8}$, respectively (Figure 1). PEG-( $\left.\mathrm{NH}_{2}\right)_{8}$ was prepared 
in high yield from PEG-(OH) 8 by first converting the hydroxyl groups in their mesylate esters followed by a reaction with ammonia. ${ }^{20}$ The conversion into amine end groups was confirmed by the appearance of a triplet at $2.94 \mathrm{ppm}$ of the $-\mathrm{CH}_{2} \mathrm{CH}_{2} \mathrm{NH}_{2}$ protons in the ${ }^{1} \mathrm{H}$ NMR spectrum (data not shown).

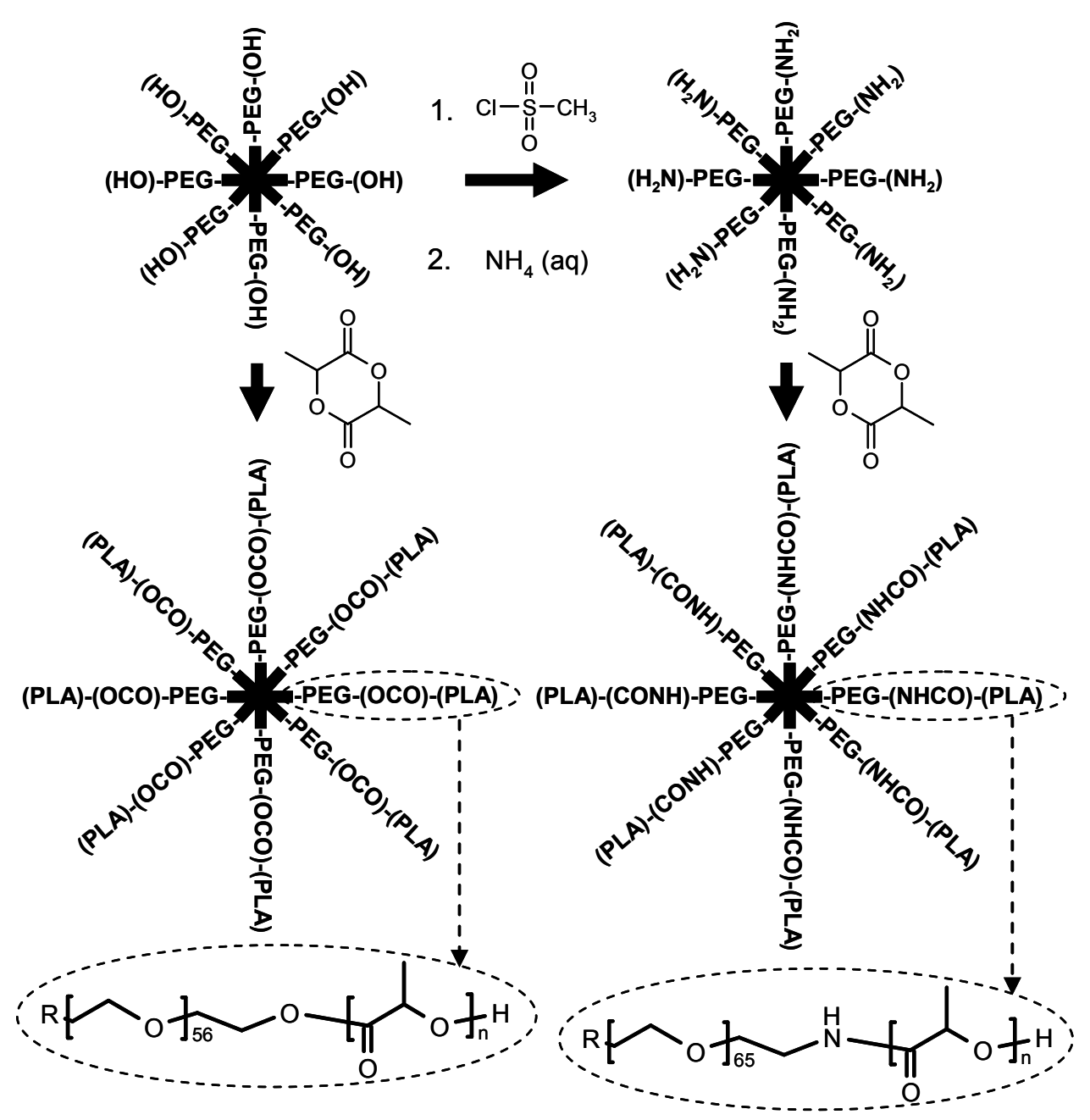

Figure 1. Synthesis scheme for the preparation of PEG-(NHCO)-(PLA) 8 and PEG-(OCO)-(PLA) 8 star block copolymers.

Similarly as PEG- $(\mathrm{OH})_{8}{ }^{9,17}$ the PEG- $\left(\mathrm{NH}_{2}\right)_{8}$ was used as an initiator in the stannous octoate catalyzed ROP of L-lactide in toluene at $110{ }^{\circ} \mathrm{C}$. The average PLA block length was calculated from the ${ }^{1} \mathrm{H}$ NMR spectrum using the integrals of peaks corresponding to the methine protons of the lactyl units and the main chain protons of PEG (Figure 2). A signal at $6.5 \mathrm{ppm}$ showed the presence of amide groups, whereas no peaks were found relating to unreacted amine groups, indicating that each of the 8 arms initiated the ROP of L-lactide. The ${ }^{1} \mathrm{H}$ NMR spectrum of PEG-(OCO)-(PLA 13$)_{8}$ showed the characteristic multiplet at $4.29 \mathrm{ppm}$ of the terminal methylene protons in the linking ester unit. 


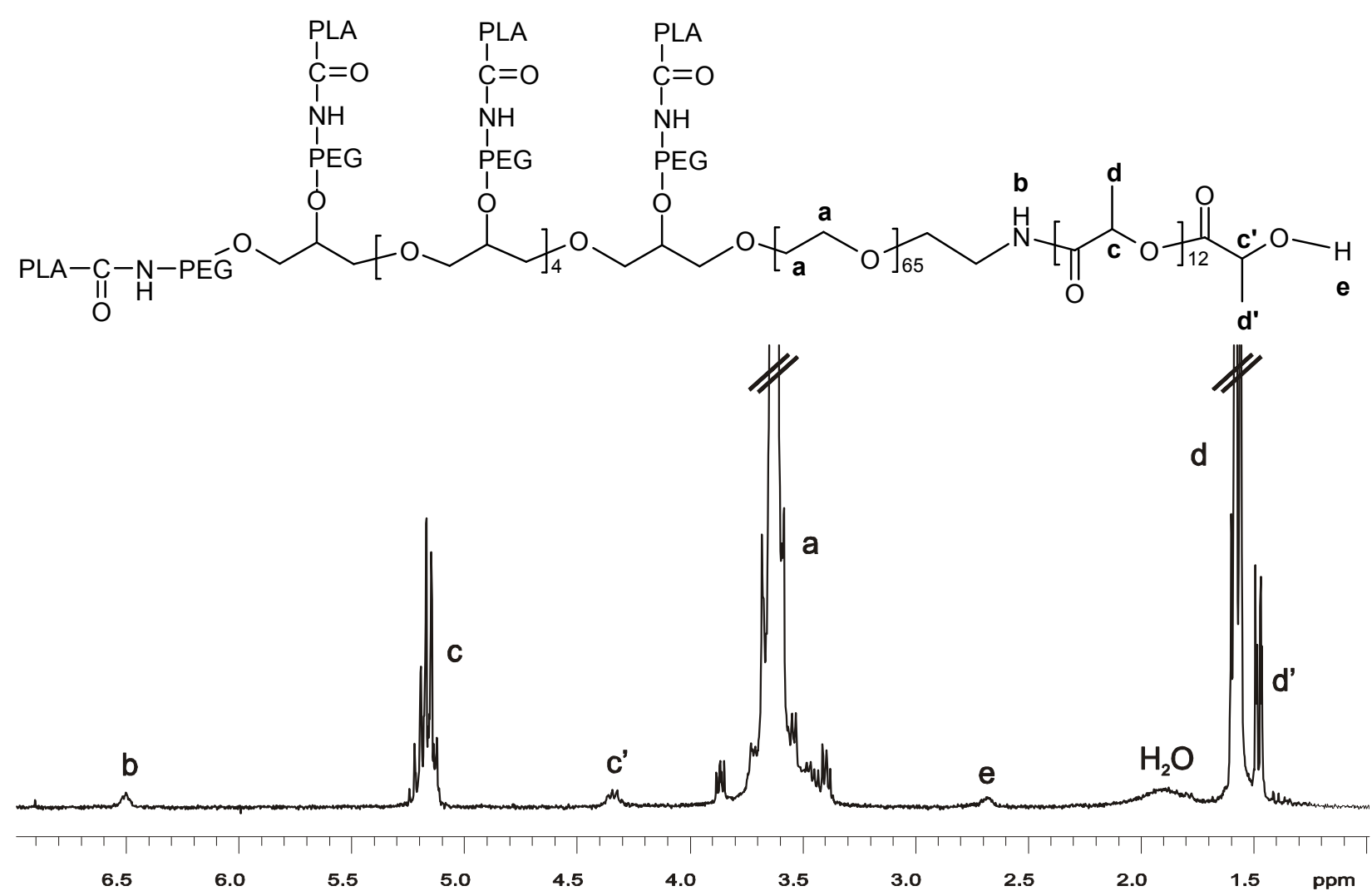

Figure 2. ${ }^{1} \mathrm{H}$ NMR spectrum of PEG-(NHCO)-(PLA 13$)_{8}$. Solvent: $\mathrm{CDCl}_{3}$.

Table 1. Characterization of PEG initiators and PEG-PLA star block copolymers.

\begin{tabular}{|c|c|c|c|c|c|c|c|}
\hline \multirow[b]{2}{*}{ Polymer } & \multicolumn{2}{|c|}{${ }^{1} \mathrm{H}$ NMR } & \multirow[b]{2}{*}{$\begin{array}{c}\eta_{\text {intr }} \\
(\mathrm{dl} / \mathrm{g})\end{array}$} & \multirow[b]{2}{*}{$\begin{array}{l}\mathrm{T}_{\mathrm{m}} \\
\left({ }^{\circ} \mathrm{C}\right)\end{array}$} & \multirow[b]{2}{*}{$\begin{array}{l}\Delta \mathrm{H}_{\mathrm{m}} \\
(\mathrm{J} / \mathrm{g})\end{array}$} & \multirow[b]{2}{*}{$\begin{array}{c}\mathrm{T}_{\mathrm{c}} \\
\left({ }^{\circ} \mathrm{C}\right)\end{array}$} & \multirow[b]{2}{*}{$\begin{array}{l}\Delta \mathrm{H}_{\mathrm{c}} \\
(\mathrm{J} / \mathrm{g})\end{array}$} \\
\hline & $\mathrm{DP}^{a}$ & $\begin{array}{c}\mathrm{M}_{\mathrm{n}} \\
(\mathrm{kg} / \mathrm{mol})\end{array}$ & & & & & \\
\hline$\overline{\text { PEG-}\left(\mathrm{NH}_{2}\right)_{8}}$ & - & 23.7 & - & 53 & 126 & 28 & 118 \\
\hline PEG-(NHCO)-(PLA 11$)_{8}$ & 10.8 & 30.0 & 0.37 & 47 & 88 & 8 & 61 \\
\hline PEG-(NHCO)-(PLA 13$)_{8}$ & 13.3 & 31.4 & 0.39 & 39 & 51 & -4 & 55 \\
\hline PEG-(NHCO)-(PLA 15$)_{8}$ & 14.7 & 32.2 & 0.47 & 38 & 48 & -20 & 51 \\
\hline 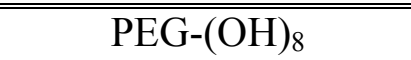 & - & 20.6 & - & 54 & 141 & 31 & 126 \\
\hline PEG-(OCO)-(PLA 13$)_{8}$ & 13.3 & 28.3 & 0.32 & 40 & 53 & 15 & 51 \\
\hline
\end{tabular}

${ }^{a}$ Degree of polymerization of the PLA blocks, expressed in lactyl units per arm.

The thermal properties of all block copolymers as determined with differential scanning calorimetry (DSC) revealed a single transition in both the second heating and the cooling scan, corresponding to melting and crystallization of the PEG domains, respectively (Table 1). No melting endotherms were detected at higher temperatures, indicating that no crystallization of the PLA phase took place. All PEG-PLA star block copolymers exhibit lower melting transitions and 
accompanying enthalpies than their PEG precursors. Apparently, the crystallization of PEG is hampered by the presence of PLA blocks.

No large differences were observed between the melting transitions and enthalpies of the PEG$(\mathrm{NHCO})-\left(\mathrm{PLA}_{13}\right)_{8}$ and PEG-(OCO)-( $\left.\mathrm{PLA}_{13}\right)_{8}$ polymers. However, PEG-(OCO)-(PLA 13$)_{8}$ crystallizes at a significantly higher temperature. Possibly the rigidity of the amide linkages, which are preferably in a trans configuration, hampers crystallization of the PEG chains.

Gelation behavior. Critical association concentration (CAC) values of the star copolymers in aqueous solution were determined with the solubilization method using the hydrophobic dye 1,6diphenyl-1,3,5-hexatriene (Table 2). ${ }^{21}$ As expected, the CAC values decrease with increasing PLA block length. The CAC values of PEG-(OCO)-(PLA $\left.{ }_{13}\right)_{8}$ and PEG-(NHCO)-(PLA 13$)_{8}$ are approximately the same, indicating that the linkage between the PEG and PLA blocks does not have a large influence on the tendency of the polymers to aggregate. The results indicate that at low concentrations all star copolymers form micelles or small aggregates with a hydrophobic PLA interior surrounded by a PEG shell. ${ }^{17,23}$

Table 2. Critical association concentrations (CAC) and critical gel concentrations (CGC) of PEGPLA star block copolymers at room temperature.

\begin{tabular}{|c|c|c|}
\hline Star block copolymer & $\begin{array}{c}\text { CAC } \\
(\mathrm{w} / \mathrm{v} \%)\end{array}$ & $\begin{array}{c}\text { CGC } \\
(\mathrm{w} / \mathrm{v} \%)\end{array}$ \\
\hline PEG-(NHCO)-(PLA 11$)_{8}$ & 0.48 & 12 \\
\hline PEG-(NHCO)-(PLA $\left.{ }_{13}\right)_{8}$ & 0.24 & 10 \\
\hline PEG-(NHCO)-(PLA $\left.{ }_{15}\right)_{8}$ & 0.19 & 7 \\
\hline \hline PEG-(OCO)-(PLA 13$)_{8}$ & 0.21 & 20 \\
\hline
\end{tabular}

In water all polymers form hydrogels above the CGC. The PEG-(NHCO)-( PLA $\left._{n}\right)_{8}$ polymers yielded translucent gels, whereas the PEG-(OCO)-( $\left.\mathrm{PLA}_{13}\right)_{8}$ polymers formed white, opaque hydrogels. It is thought that the translucency of hydrogels based on PEG-(NHCO)-(PLA) 8 star block copolymers may be the result of phase separation between the PEG and PLA phases into smaller domains as compared to the PEG-(OCO)-(PLA) 8 star block copolymer. The CGC of PEG(OCO)-(PLA 13$)_{8}$ was significantly higher $(20 \mathrm{w} / \mathrm{v} \%)$ than the CGC of PEG-(NHCO)-(PLA $\left.)_{13}\right)_{8}(10$ w/v \%) (Table 2).

The temperature dependent gelation behavior, determined with the vial tilting method in the range of 2 to $80{ }^{\circ} \mathrm{C}$ (Figure 3), shows that both PEG-(NHCO)-(PLA) 8 and PEG-(OCO)-(PLA $\left.{ }_{13}\right)_{8}$ exhibit a reversible phase transition from the gel to the sol state. Moreover, changing the linkage between PEG and PLA from an ester to an amide group resulted in a shift of the boundary curve to lower 
concentrations. Upon increasing the hydrophobic block length of PEG-(NHCO)-(PLA) 8 copolymers, the gel to sol transitions are shifted to higher temperatures due to increased hydrophobic interactions. At higher concentrations, the gel to sol transition shifts to higher temperatures due to an increased number of physical crosslinks between micelles.

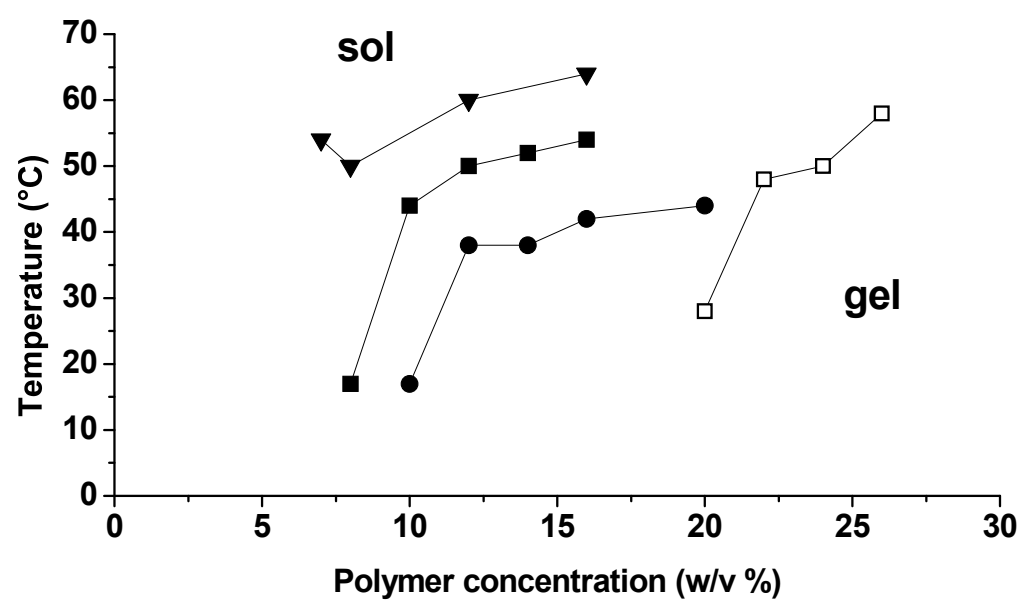

Figure 3. Gel-sol transition temperatures of PEG-PLA star block copolymers in water determined with the vial tilting method. PEG-(NHCO)-(PLA $\left.)_{11}\right)_{8}(\bullet)$, PEG-(NHCO)-(PLA 13$)_{8}$ (匹), PEG$(\mathrm{NHCO})-\left(\mathrm{PLA}_{15}\right)_{8}(\boldsymbol{\nabla})$, PEG-(OCO)-( $\left.\mathrm{PLA}_{13}\right)_{8}(\square)$.

NMR analysis of hydrogels. The ${ }^{1} \mathrm{H}$ spectra of PEG-(NHCO)-(PLA $\left.{ }_{13}\right)_{8}$ and PEG-(OCO)$\left(\mathrm{PLA}_{13}\right)_{8}$ in $\mathrm{D}_{2} \mathrm{O}(12 \mathrm{w} / \mathrm{v} \%$ and $22 \mathrm{w} / \mathrm{v} \%$, respectively) at different temperatures in the gel and sol phases (Figure 4) show distinguishable peaks ascribed to $\mathrm{CH}(5.23 \mathrm{ppm})$ and $\mathrm{CH}_{3}(1.43$ and 1.56 ppm) groups of PLA and to $\mathrm{CH}_{2}$ groups (3.68 ppm) of PEG. Broader lines are observed for PEG(OCO)-(PLA $\left.{ }_{13}\right)_{8}$ due to the higher polymer concentration. The HDO signal shifts upfield with increasing temperature, as commonly found. ${ }^{24}$ Two signals are distinguishable for the PLA methyl

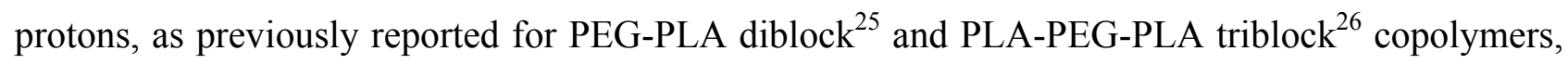
which can be ascribed to the presence of different chemical environments within a relatively mobile interfacial region. At room temperature (Figure 4a), the intensities of the PLA peaks in the spectrum are lower than expected on the basis of stoichiometry with respect to the methylene peaks of the PEG chain. Instead all the PEG $\mathrm{CH}_{2}$ protons at all temperatures are detected, as inferred from a comparison with an external standard. With increasing temperature, variations in the relative intensities of the PLA and PEG signals are observed (Figure $4 b$ ). 
(a)
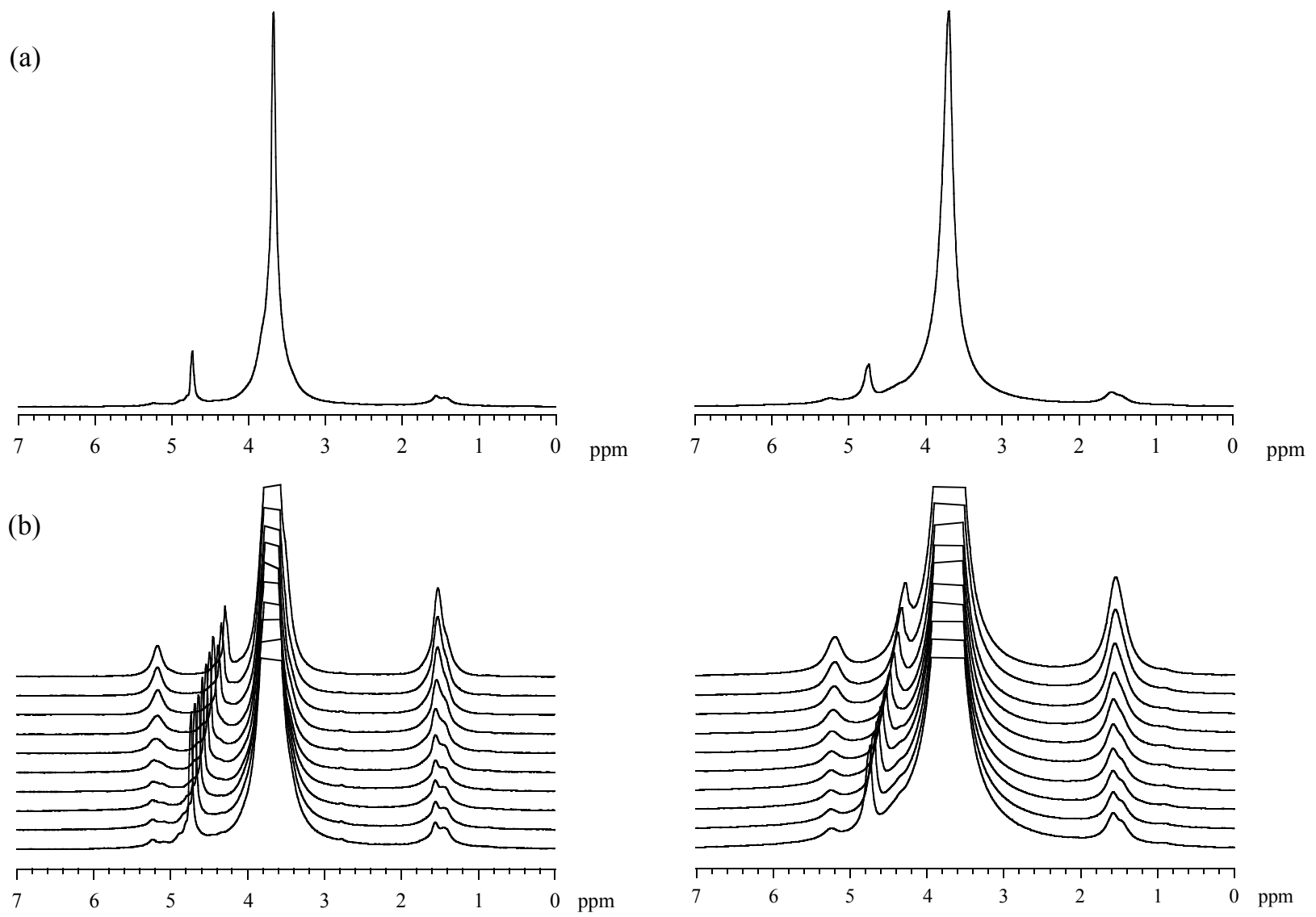

Figure 4. ${ }^{1} \mathrm{H}$ NMR spectra of (left) PEG-(NHCO)-(PLA $\left.{ }_{13}\right)_{8}(12 \mathrm{w} / \mathrm{v} \%)$ and (right) PEG-(OCO)$\left(\mathrm{PLA}_{13}\right)_{8}\left(22 \mathrm{w} / \mathrm{v} \%\right.$ ) in $\mathrm{D}_{2} \mathrm{O}$ : (a) full spectrum at $25^{\circ} \mathrm{C}$; (b) spectra recorded from $25^{\circ} \mathrm{C}$ (bottom spectrum) to $70{ }^{\circ} \mathrm{C}$ (top spectrum) with $5{ }^{\circ} \mathrm{C}$ increments are shown with an expanded vertical scale.

As shown in Figure 5, as the temperature increases the PLA peak intensities increase and show an inflection in the region of the gel to sol transition. However the peak intensities do not reach, even at the highest temperature, the values expected on the basis of stoichiometry with respect to the PEG methylene protons (i.e. 0.051 for $\mathrm{CH}$ and 0.146 for $\mathrm{CH}_{3}$ in the case of PEG-(NHCO)$\left(\mathrm{PLA}_{13}\right)_{8} ; 0.059$ for $\mathrm{CH}$ and 0.169 for $\mathrm{CH}_{3}$ in the case of PEG-(OCO)-(PLA $\left.\left.{ }_{13}\right)_{8}\right)$. In particular, spectral deconvolution of the methyl signal (Figure 4) showed that, with increasing temperature, the intensity of the $\mathrm{CH}_{3}$ peak at 1.56 ppm steadily increases, whereas the intensity of the peak at 1.43 remains nearly constant. 

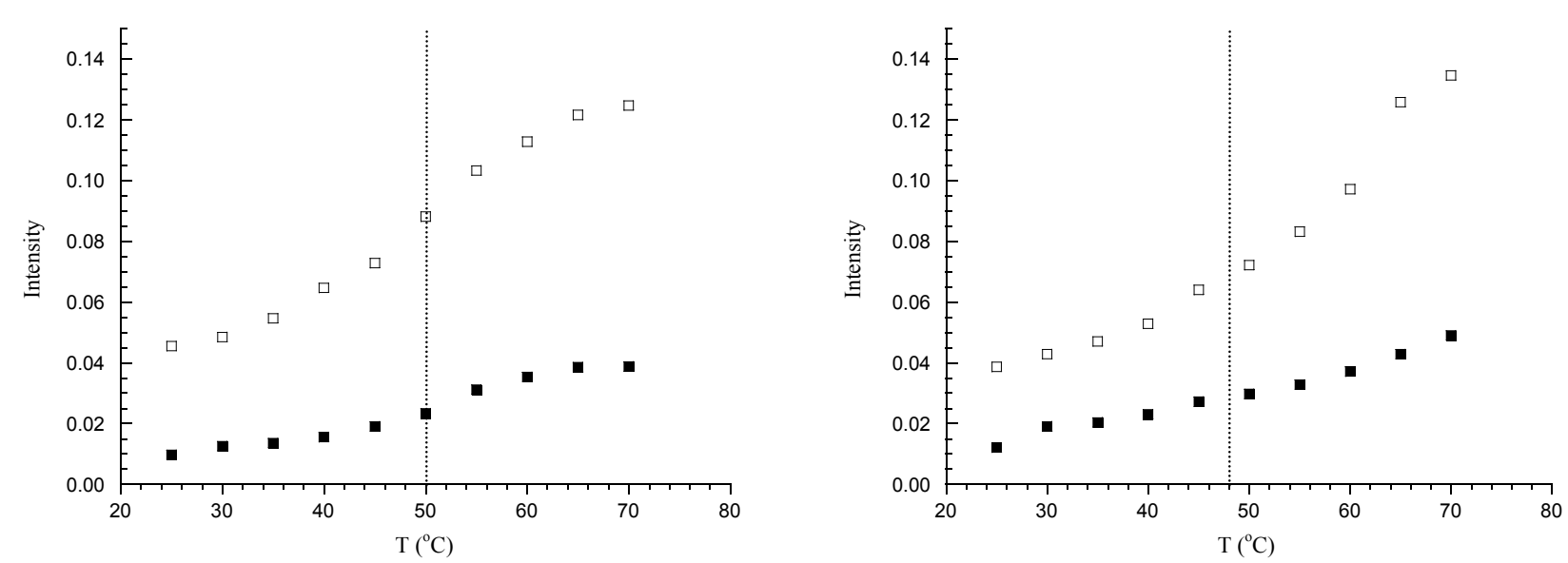

Figure 5. Temperature dependence of the relative intensities of ${ }^{1} \mathrm{H}$ NMR peaks of $\mathrm{CH}(\mathbf{\square})$ and $\mathrm{CH}_{3}$ ( $\square$ ) of PLA in (left) PEG-(NHCO)-(PLA $\left.{ }_{13}\right)_{8}(12 \mathrm{w} / \mathrm{v} \%)$ and (right) PEG-(OCO)-(PLA 13$)_{8}(22 \mathrm{w} / \mathrm{v}$ $\%)$ in $\mathrm{D}_{2} \mathrm{O}$. Intensities are normalized with respect to the PEG methylene proton peak intensity. The dotted line represents the gel to sol transition as determined with the vial tilting method.

The observations on the relative signal intensities of PEG and PLA protons are similar to those reported in literature for PEG-PLA block copolymers ${ }^{25,27,28}$ and can be ascribed to the formation of hydrophobic PLA aggregates with sensibly lower mobility with respect to the hydrophilic PEG chains. For both PEG-(NHCO)-(PLA 13$)_{8}$ and PEG-(OCO)-(PLA 13$)_{8}$ the ${ }^{1} \mathrm{H}$ NMR data clearly show that over $70 \%$ of the PLA units are quite rigid at room temperature in the gel phase, displaying however a significant increase in mobility with increasing temperatures up to the gel-sol transition. In the sol phase the fraction of mobile PLA units continues to increase reaching about $80 \%$ at the highest temperature. All the above findings concur on a description of hydrated PEG-PLA polymer systems where two types of domains are present, with rigid hydrophobic PLA aggregates surrounded by hydrophilic mobile PEG chains and water, with a relatively mobile interfacial region containing about $20 \%$ of the PLA units. The main effect of heating is an increase of the mobility of the PLA units within both the gel and sol phases, with a larger variation around the transition temperature. 
Rheology. The storage (G') and loss (G') modulus of hydrogels at various polymer concentrations were determined with oscillatory rheology measurements at $20{ }^{\circ} \mathrm{C}$ (Figure 6). For all copolymers, G' exceeded G', confirming that the systems were in the gel state. ${ }^{29}$ It is apparent from Figure 6a that replacing the ester group between the PEG and PLA blocks with an amide group results in a marked increase in the storage modulus. As an example, a $20 \mathrm{w} / \mathrm{v} \%$ PEG-(OCO)$\left(\mathrm{PLA}_{13}\right)_{8}$ hydrogel has a storage modulus of $6.9 \mathrm{kPa}$ whereas a $20 \mathrm{w} / \mathrm{v} \%$ PEG-(NHCO)-(PLA 13$)_{8}$ hydrogel has a storage modulus of $17 \mathrm{kPa}$. Possibly, the rigidity of the amide groups results in restricted conformational freedom leading to more effective physical crosslinks between hydrophobic domains. This is supported by the presence of larger aggregates in PEG-(NHCO)$(\mathrm{PLA})_{8}$ star block copolymer solutions as compared to PEG-(OCO)-(PLA) 8 star block copolymer solutions (vide infra) and may be a reason for the enhanced mechanical properties. Furthermore, it can be observed that the gel stiffness is strongly dependent on the hydrophobic block length. Increasing the number of lactyl units from 11 to 13 results in an increase in $\mathrm{G}^{\prime}$ from 6.2 to $17 \mathrm{kPa}$ for $20 \mathrm{w} / \mathrm{v} \%$ PEG-(NHCO)-(PLA $\left.{ }_{13}\right)_{8}$ hydrogels, probably due to increased hydrophobic interactions between the PLA arms. The increase in G' with concentration can be ascribed to the formation of a more densely physically crosslinked network. These rheological experiments indicate that hydrogels can be designed with storage moduli up to $27 \mathrm{kPa}$ by varying the concentration and the hydrophobic chain length.
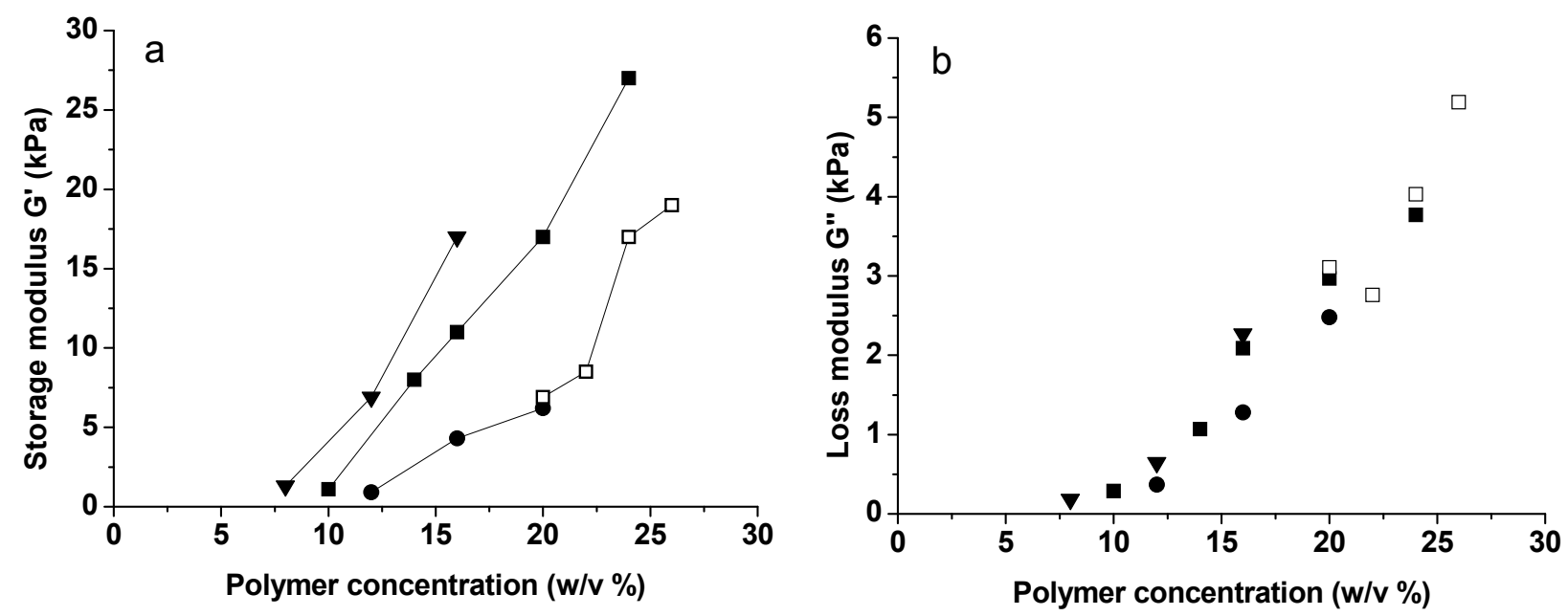

Figure 6. Storage modulus (G') (a) and loss modulus (G',) (b) at $20{ }^{\circ} \mathrm{C}$ versus concentration for hydrogels prepared from various PEG-PLA star block copolymers. PEG-(NHCO)-(PLA $\left.{ }_{11}\right)_{8}(\bullet)$, PEG-(NHCO)-(PLA 13$)_{8}(\boldsymbol{\bullet})$, PEG-(NHCO)-(PLA 15$)_{8}(\boldsymbol{\nabla})$, PEG-(OCO)-(PLA 13$)_{8}(\square)$.

The temperature dependent gel-sol transition of the hydrogels in the temperature range of 20 to 70 ${ }^{\circ} \mathrm{C}$ was determined from the crossover point of $\mathrm{G}$ ' and $\mathrm{G}$ '. The gel-sol transition temperatures as presented in Figure 7 clearly show the large differences in the thermal stability of the hydrogels. 
The gel-sol transition temperatures for $14 \mathrm{w} / \mathrm{v} \%$ PEG-(NHCO)-(PLA $\left.{ }_{13}\right)_{8}$ and $20 \mathrm{w} / \mathrm{v} \%$ PEG(OCO)-(PLA $\left.{ }_{13}\right)_{8}$ hydrogels were $53{ }^{\circ} \mathrm{C}$ and $29{ }^{\circ} \mathrm{C}$, respectively. These temperatures are in good accordance with the gel-sol transitions found via the vial tilting method as shown in Figure 3. G' and G' showed little variation when an amplitude sweep from 0.1 to $10 \%$ was performed at the end of each rheological experiment, indicating that a strain of $0.5 \%$ is within the linear viscoelastic range of the hydrogels.

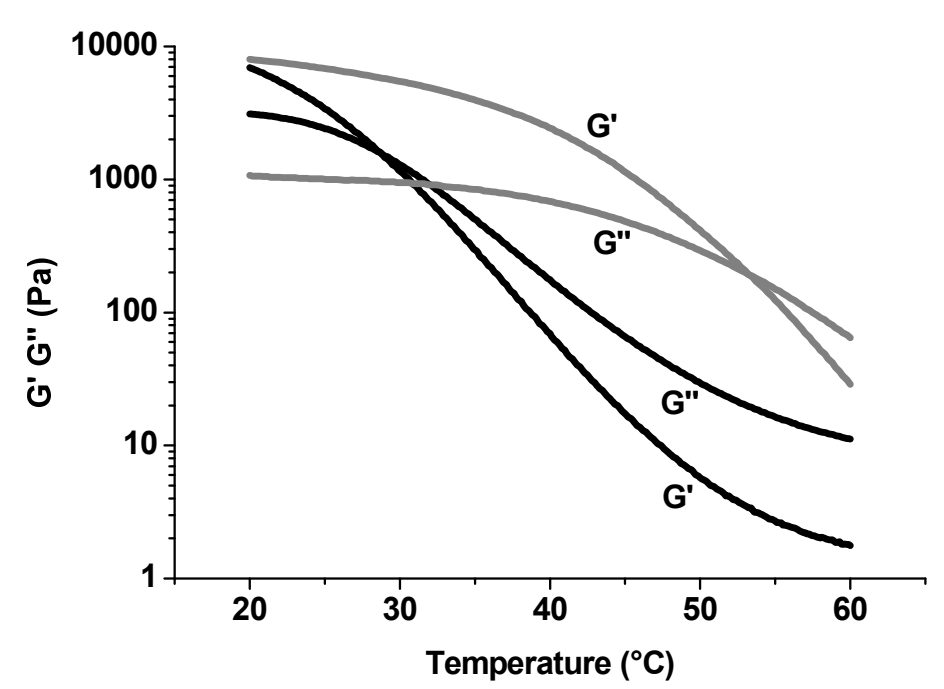

Figure 7. Storage $\left(G^{\prime}\right)$ and loss modulus $\left(G^{\prime} \text { ') of } 14 \text { w/v \% PEG-(NHCO)-(PLA } 13\right)_{8}$ (grey lines) and $20 \mathrm{w} / \mathrm{v} \%$ PEG-(OCO)-( $\left.\mathrm{PLA}_{13}\right)_{8}$ (black lines) hydrogels upon heating from 20 to $60{ }^{\circ} \mathrm{C}$.

Aggregate size and size distribution. The temperature dependent gelation of the star block copolymers results from a close packing of micelles or small aggregates upon an increase in hydrophobicity due to partial dehydration of the PEG chains. When the total aggregate volume fraction exceeds the maximum packing fraction gelation will occur. ${ }^{30}$ Using dynamic light scattering (DLS) the aggregate size and aggregate size distributions of the star copolymers in 0.5 w/v \% aqueous solutions were determined. As depicted in Figure 8 two size distributions are present in all PEG-(NHCO)-( $\left.\mathrm{PLA}_{n}\right)_{8}$ solutions. The results show that these materials form micelles as well as larger sized aggregates at concentrations above the CAC. Small aggregates with diameters ranging from 20-40 $\mathrm{nm}$ and larger aggregates with an average diameter of $100 \mathrm{~nm}$ are present. An increase in the hydrophobic block length shifts the distribution to larger aggregates. In contrast, PEG-(OCO)-( $\left.\mathrm{PLA}_{13}\right)_{8}$ shows only one distribution with an average diameter of approximately $25 \mathrm{~nm}$, indicative of micellar type structures. ${ }^{31}$ At low concentrations, all star copolymers form aggregates with a hydrophobic PLA interior surrounded by a PEG shell. The increased rigidity of a linking amide group as compared to a linking ester group may hamper individual molecules to adapt their micellar conformation, resulting in larger aggregates. 

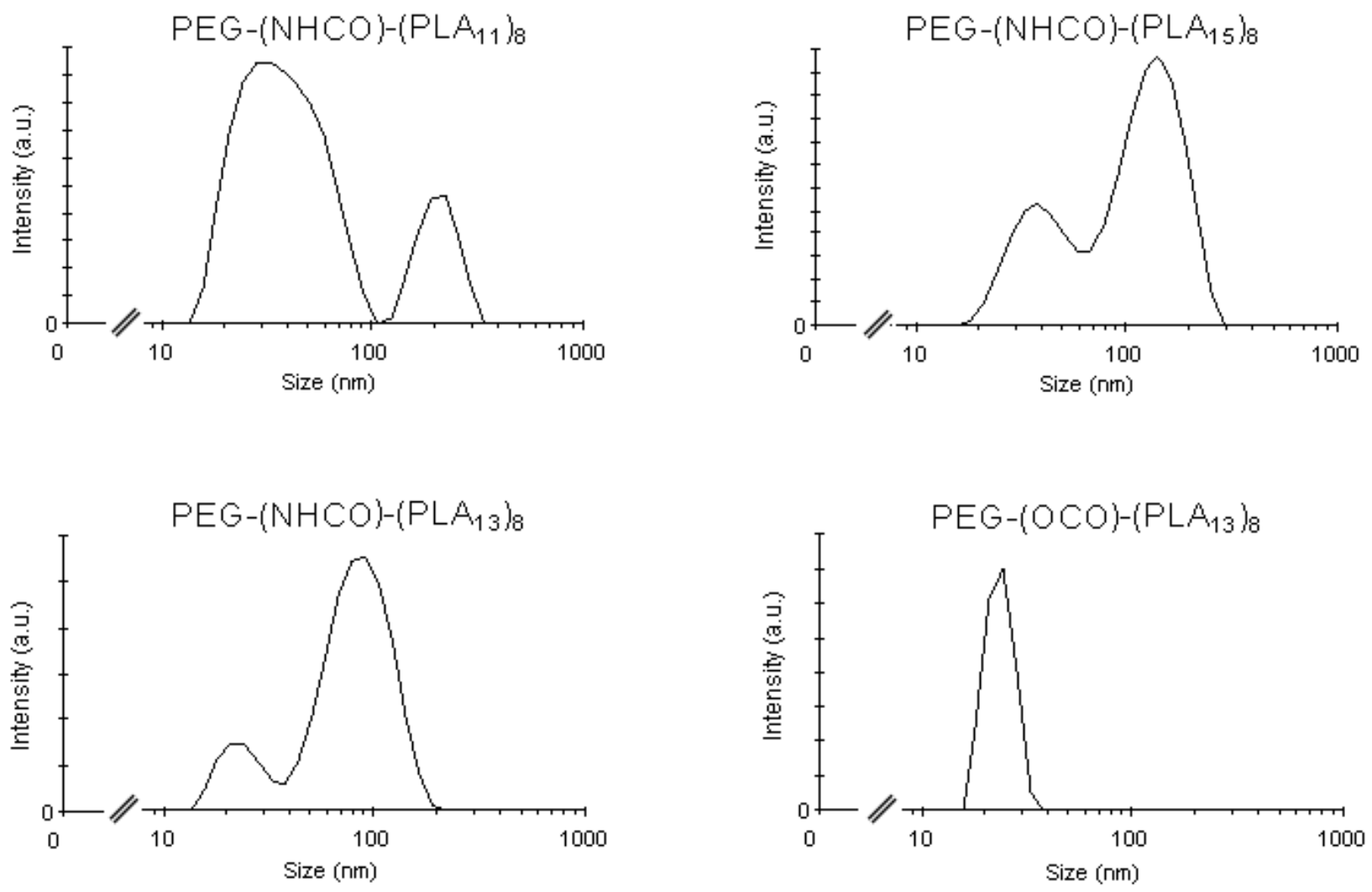

Figure 8. Aggregate size distributions of $0.5 \mathrm{w} / \mathrm{v} \%$ aqueous PEG-(NHCO)-(PLA $)_{8}$ and PEG(OCO)-( $\left(\mathrm{PLA}_{13}\right)_{8}$ copolymer solutions at room temperature.

The presence of the larger aggregates in the PEG-(NHCO)-( $\left.\mathrm{PLA}_{13}\right)_{8}$ star block copolymer solutions may be a reason for the shift of the reversible gel to sol transitions to lower concentrations. This is also reflected in the gel window of the PEG-(NHCO)-(PLA 13$)_{8}$ copolymer, which is much larger, compared to the PEG-(OCO)-( $\left.\mathrm{PLA}_{13}\right)_{8}$ copolymer (Figure 3$)$.

It should be noted that the intensity of scattered light cannot directly be related to the number of aggregates, because larger aggregates scatter light more intensely than smaller aggregates. 
Hydrogel degradation/dissolution. The in vitro stability of various hydrogels was investigated by determining the mass of dissolved/degraded copolymer that diffused into the supernatant PBS at $37{ }^{\circ} \mathrm{C}$ in time (Figure 9). It was observed that the $22 \mathrm{w} / \mathrm{v} \%$ PEG-(OCO)-(PLA $\left.{ }_{13}\right)_{8}$ hydrogel completely solubilized within $5 \mathrm{~d}$. The $22 \mathrm{w} / \mathrm{v} \%$ PEG-(NHCO)-(PLA 13$)_{8}$ hydrogel exhibits a significantly improved stability, and complete degradation/dissolution is only observed at day 15 . These hydrogels may loose their structural integrity because the micellar and aggregate packing is disrupted due to the concentration difference of the copolymer in the hydrogel and supernatant buffer. Increasing the polymer concentration from 12 to $22 \mathrm{w} / \mathrm{v} \%$ in the PEG-(NHCO)-(PLA 13$)_{8}$ hydrogel results in prolonged stability, as does increasing the number of lactyl units per PLA arm from 13 to 15 . A higher physical crosslink density and increased hydrophobic interactions are most likely the reasons for this increased stability.

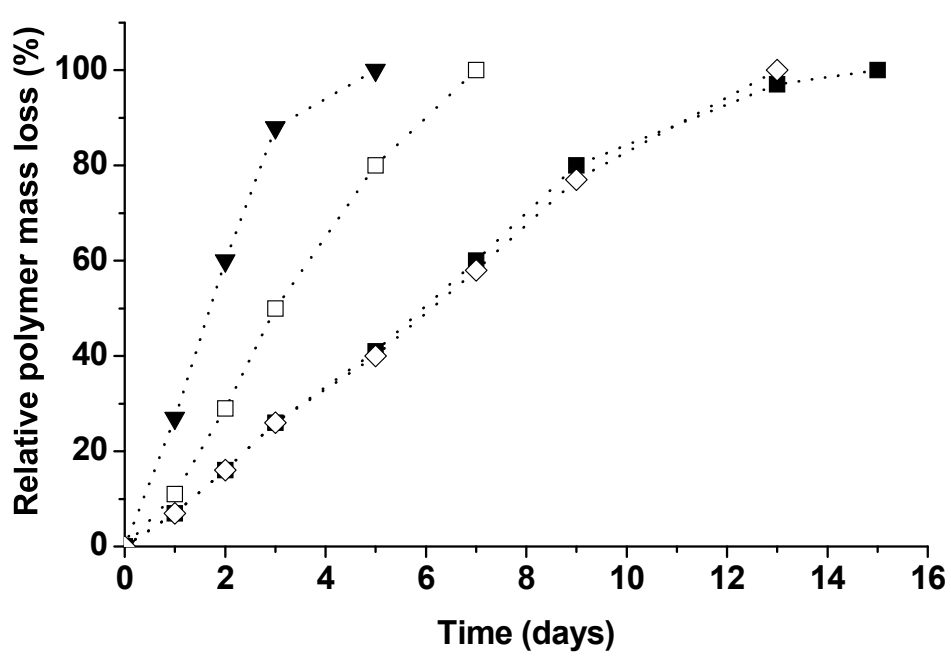

Figure 9. Relative mass loss of PEG-(NHCO)-(PLA $)_{8}$ and PEG-(OCO)-(PLA 13$)_{8}$ hydrogels in PBS at $37^{\circ} \mathrm{C} .22 \mathrm{w} / \mathrm{v} \%$ PEG-(OCO)-(PLA 13$)_{8}(\boldsymbol{\nabla}), 12 \mathrm{w} / \mathrm{v} \%$ PEG-(NHCO)-(PLA 13$)_{8}(\square), 22 \mathrm{w} / \mathrm{v}$ $\%$ PEG-(NHCO)-(PLA 13$)_{8}(\boldsymbol{\varpi}), 12$ w/v \% PEG-(NHCO)-(PLA $\left.{ }_{15}\right)_{8}(\diamond)$.

The remarkable difference in degradation rates of the star block copolymers having an ester or amide linkage between the PEG and PLA blocks prompted us to investigate the degradation/dissolution mechanism in detail. The degraded and dissolved material released in the PBS degradation medium was analyzed by ${ }^{1} \mathrm{H}$ NMR spectroscopy. In Figure 10 the spectrum of a fully solubilized PEG-(NHCO)-(PLA $\left.{ }_{15}\right)_{8}$ hydrogel sample after $13 \mathrm{~d}$ at $37{ }^{\circ} \mathrm{C}$ in PBS is presented together with the spectra of the starting star block copolymer and an almost fully degraded sample of PEG-(NHCO)-(PLA 15$)_{8}$. The fully degraded PEG-(NHCO)-(PLA 15$)_{8}$ was prepared by dissolving the copolymer in 2-(cyclohexylamino)ethanesulfonic acid (CHES) buffer at pH 10.4 and stirring for $18 \mathrm{~h}$ at room temperature. Similar conditions have been reported to cause rapid degradation of PLA. $^{32}$ 

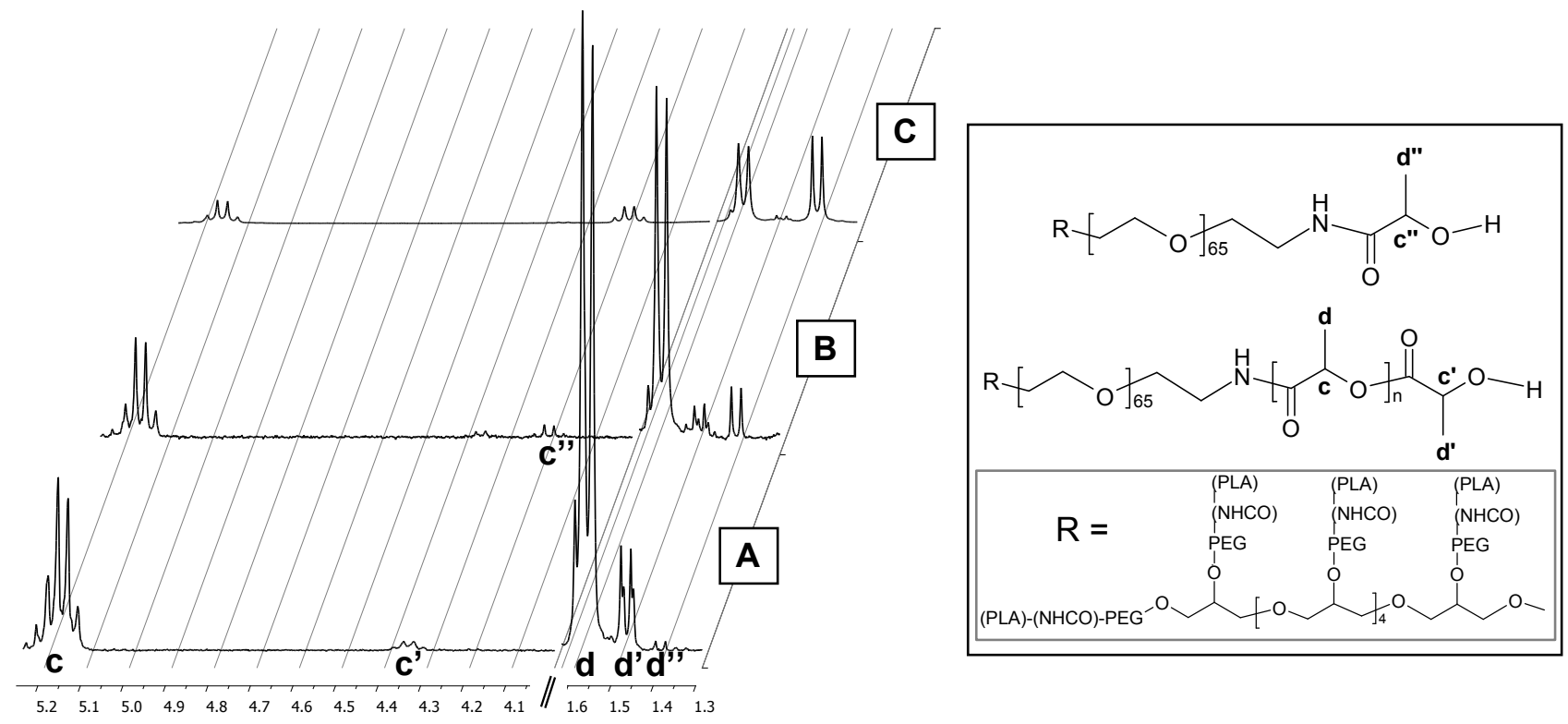

Figure 10. ${ }^{1} \mathrm{H}$ NMR spectra of 3 PEG-(NHCO)-(PLA 15$)_{8}$ samples: PEG-(NHCO)-(PLA 15$)_{8}(\mathrm{~A})$, a fully solubilized PEG-(NHCO)-(PLA 15$)_{8}$ hydrogel (B) and PEG-(NHCO)-(PLA 15$)_{8}$ that was stirred in CHES buffer for $18 \mathrm{~h}$ at $\mathrm{pH} 10.4(\mathrm{C})$. Solvent: $\mathrm{CDCl}_{3}$.

By comparing Figure 10A and Figure 10B, it can be seen that the ratio of the peak areas of nonterminal lactyl protons to terminal lactyl protons (c to $\mathbf{c}^{\prime}+\mathbf{c}$ '" and $\mathbf{d}$ to $\mathbf{d}$ ' $+\mathbf{d}$ ') decreases when PEG-(NHCO)-(PLA 15$)_{8}$ is degraded for $13 \mathrm{~d}$. The ratio further decreases for PEG-(NHCO)$\left(\mathrm{PLA}_{15}\right)_{8}$ that was stirred in CHES buffer at $\mathrm{pH} 10.4$ for $18 \mathrm{~h}$ (Figure 10C). The signal at $4.25 \mathrm{ppm}$ of the methine protons (c') and the signal at $1.40 \mathrm{ppm}$ of the methyl protons (d"') are characteristic for single lactyl units coupled to the PEG via an amide group. Signals typical of methylene protons next to an amine group at $2.9 \mathrm{ppm}$ were not observed in any of the spectra. These data show that the amide groups are not affected in the hydrolysis reaction. By comparing the integrals of peaks corresponding to the methine protons of PLA lactyl units and the main chain protons of PEG, it was found that the $\mathrm{M}_{\mathrm{n}}$ of the PEG-(NHCO)-(PLA $\left.{ }_{15}\right)_{8}$ star block copolymer decreased from $32.2 \mathrm{~kg} / \mathrm{mol}$ in a freshly prepared hydrogel to $28.9 \mathrm{~kg} / \mathrm{mol}$ in a fully solubilized hydrogel sample $\left(\mathrm{t}_{1}\right.$ in Figure 11). Degradation of the PEG-(NHCO)-( $\left.\mathrm{PLA}_{15}\right)_{8}$ copolymer in CHES buffer at $\mathrm{pH} 10.4$ for $18 \mathrm{~h}$ revealed a reduction to a $\mathrm{M}_{\mathrm{n}}$ of $25.0 \mathrm{~kg} / \mathrm{mol}$ corresponding to a copolymer comprising two lactyl units per arm ( $t_{2}$ in Figure 11). 


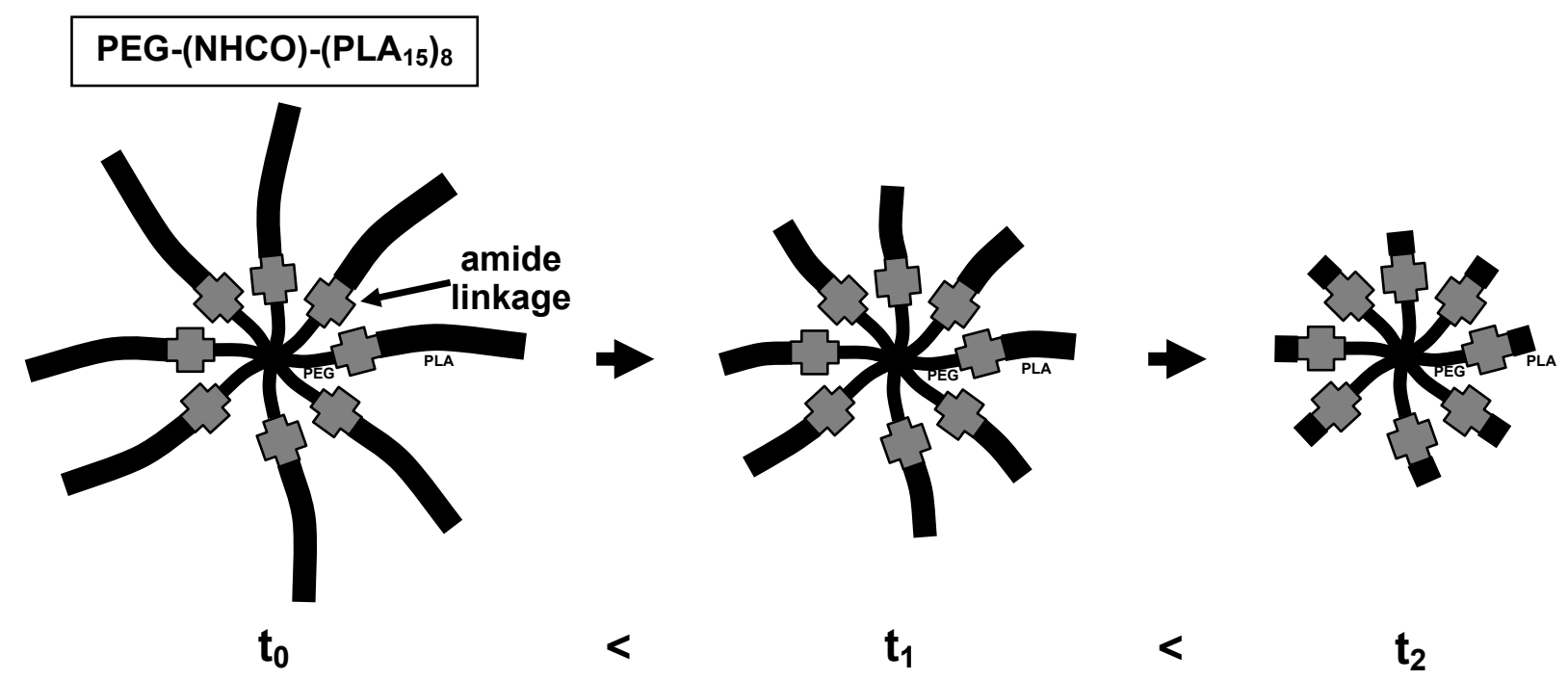

Figure 11. Schematic representation of the degradation of PEG-(NHCO)-(PLA 15$)_{8}$.

The loss of lactyl units occurs through hydrolysis of ester groups in the PLA arms. It is thought that the PLA arms degrade by intramolecular transesterification, also called backbiting, which mechanism was proposed by de Jong et al. for the degradation of lactic acid oligomers in neutral or alkaline medium. ${ }^{33}$ According to this mechanism, nucleophilic attack of the hydroxyl end group on the penultimate carbonyl group leads to the elimination of one lactide unit. It has to be noted that the PLA phase is amorphous, which leads to a rather fast degradation.

As has been previously suggested, the degradation of the PEG-PLA block copolymers proceeds through preferential hydrolysis of the linking ester bond. ${ }^{18,19}$ In Figure 12 the ${ }^{1} \mathrm{H}$ NMR spectra of a PEG-(OCO)-(PLA $\left.{ }_{13}\right)_{8}(\mathrm{~A})$ and a fully solubilized PEG-(OCO)-(PLA 13$)_{8}$ hydrogel sample after $5 \mathrm{~d}$ in PBS (B) are presented. In Figure $12 \mathrm{C}$ the ${ }^{1} \mathrm{H}$ NMR spectrum of PEG- $(\mathrm{OH})_{8}$ is depicted. Because of overlapping signals all spectra were recorded in the presence of trifluoroacetic anhydride. 


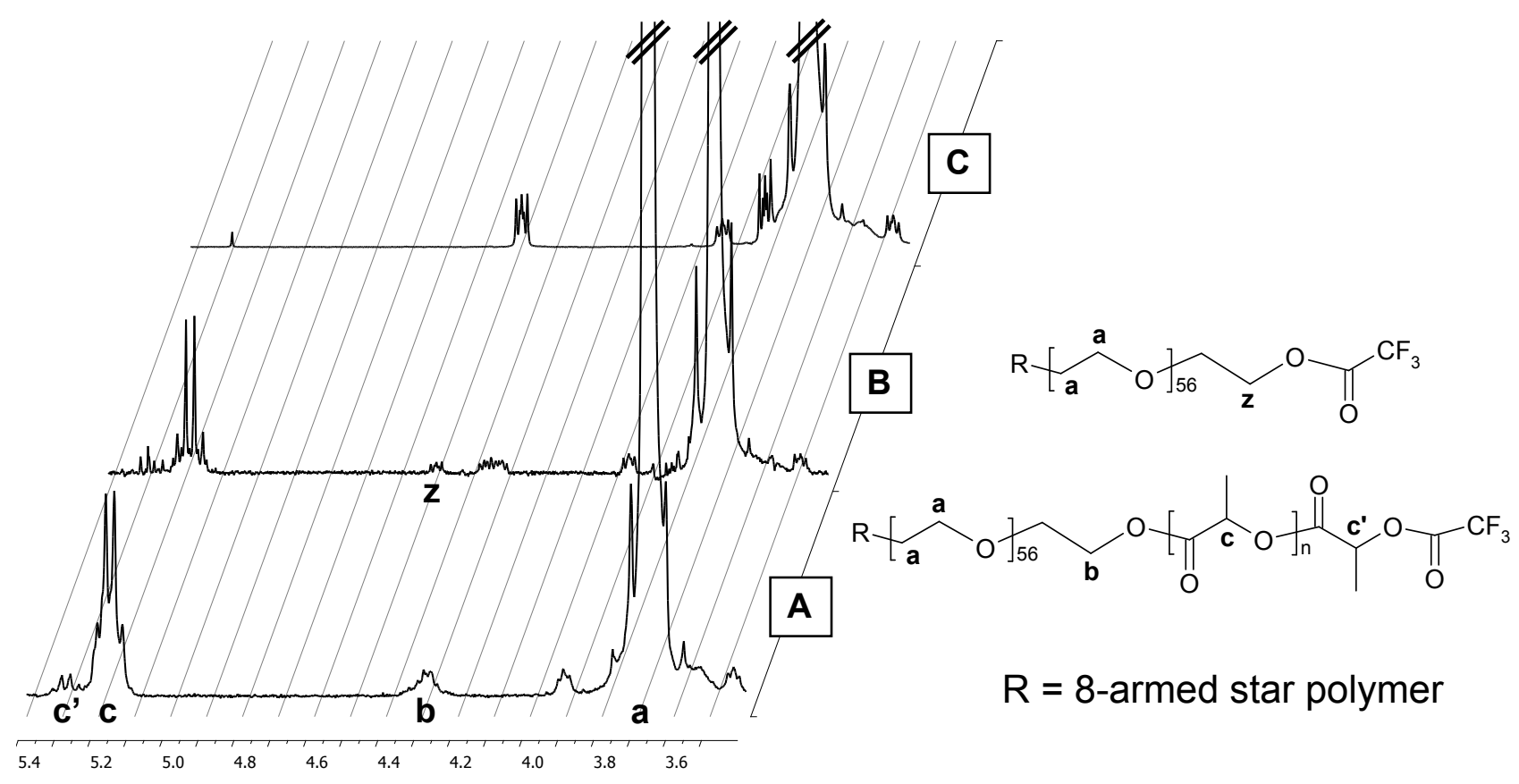

Figure 12. ${ }^{1} \mathrm{H}$ NMR spectra of PEG-(OCO)-(PLA $\left.{ }_{13}\right)_{8}(\mathrm{~A})$, a fully solubilized PEG-(OCO)-(PLA 13$)_{8}$ hydrogel $(B)$ and PEG- $(\mathrm{OH})_{8}(\mathrm{C})$. Solvent: $\mathrm{CDCl}_{3}$ in the presence of trifluoroacetic anhydride.

A hydrogel that becomes fully solubilized upon degradation (Figure 12B) revealed a signal typical of a trifluoroacetyl group linked to PEG at $4.5 \mathrm{ppm}(\mathbf{z})$. These data show that hydrolysis of a few PEG-PLA linking ester groups in the PEG-(OCO)-(PLA $\left.{ }_{13}\right)_{8}$ make the copolymer soluble in PBS (Figure 13). By comparing the relative peak integral values of the terminal PEG methylene protons and the main chain PEG protons, it was found that on average 3 PLA arms per molecule were removed. Because the ratio of the integrals of the non-terminal lactyl methine (c) and terminal lactyl methine (c') proton signals (Figure 12A and B) remained relatively constant only minor degradation has taken place in the remaining PLA arms. The $\mathrm{M}_{\mathrm{n}}$ of the PEG-(OCO)-(PLA $\left.\mathrm{P}_{13}\right)_{8}$ star block copolymer decreased from $28.3 \mathrm{~kg} / \mathrm{mol}$ in a freshly prepared hydrogel to $24.8 \mathrm{~kg} / \mathrm{mol}$ in a fully solubilized hydrogel sample ( $t_{1}$ in Figure 13$)$.
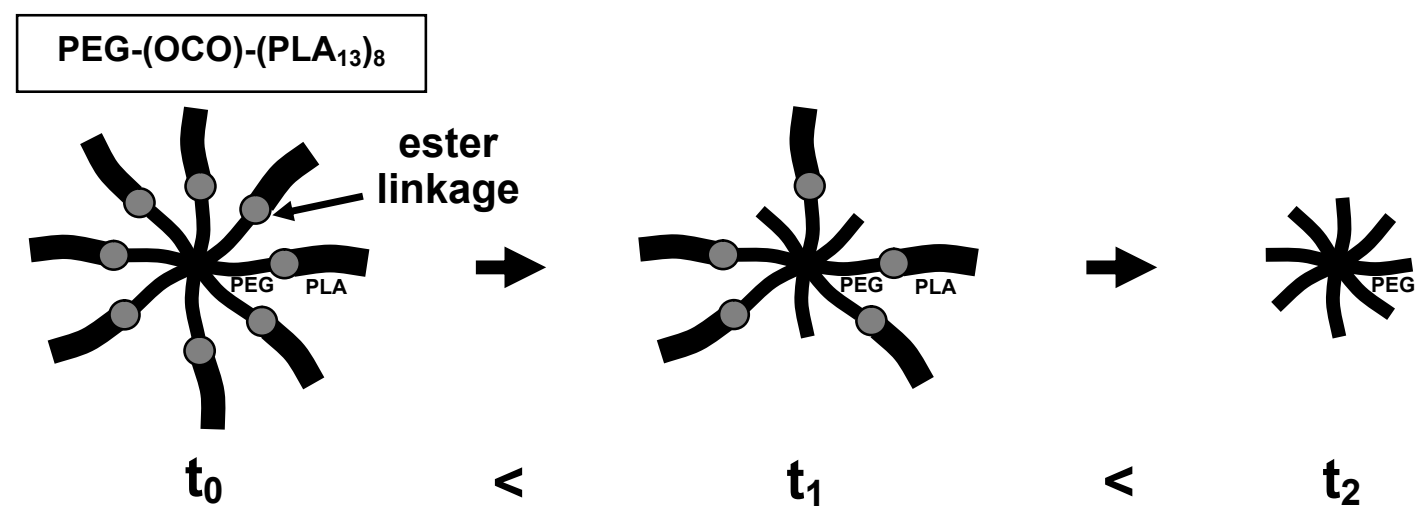

$\mathbf{t}_{1}$

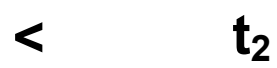

Figure 13. Schematic representation of the degradation of PEG-(OCO)-(PLA $\left.{ }_{13}\right)_{8}$. 
The PEG-(OCO)-(PLA $\left.{ }_{13}\right)_{8}$ hydrogels likely loose their structural integrity relatively fast because the micellar packing is disrupted due to the decreased hydrophobic interactions upon preferred hydrolysis of the linking ester groups.

The end group analysis as presented clearly proves the different degradation mechanisms for the ester and amide type star block copolymers and actually confirms the mechanism as was suggested in earlier literature. ${ }^{18,19}$

\subsection{Conclusions}

PEG-(NHCO)-(PLA) 8 and PEG-(OCO)-(PLA) 8 star block copolymers were prepared by ring opening polymerization of lactide initiated by PEG-(NH$)_{8}$ and PEG-(OH) $)_{8}$, respectively. Dye solubilization experiments showed that aqueous solutions of these copolymers formed micelles and aggregates at low concentrations. At higher concentrations, thermo-sensitive gels could be obtained, exhibiting a gel to sol transition upon temperature elevation. In comparison with PEG-(OCO)$(\mathrm{PLA})_{8}$ the PEG-(NHCO)-(PLA) 8 hydrogels exhibited a lower CGC and a larger gel window. Furthermore, oscillatory rheology measurements revealed that a $20 \mathrm{w} / \mathrm{v} \%$ PEG-(NHCO)-(PLA 13$)_{8}$ hydrogel exhibits a high storage modulus of $17 \mathrm{kPa}$, whereas a $20 \mathrm{w} / \mathrm{v} \%$ PEG-(OCO)-(PLA 13$)_{8}$ hydrogel has a storage modulus of $7 \mathrm{kPa}$. Hydrolytic degradation of these hydrogels revealed that the PEG-(OCO)-(PLA $\left.{ }_{13}\right)_{8}$ hydrogel becomes completely solubilized through preferential hydrolysis of linking ester groups between the PEG and PLA blocks. In contrast, the stable amide linking unit in the PEG-(NHCO)-(PLA) 8 hydrogels only allowed degradation to take place via hydrolysis of ester groups in the PLA chains affording materials which are more slowly degrading. The enhanced mechanical properties and stability towards hydrolytic degradation make the PEG-(NHCO)-(PLA) 8 hydrogels interesting for biomedical applications such as controlled drug delivery systems and matrices for tissue engineering.

\subsection{References}

[1] Zisch, A. H.; Lutolf, M. P.; Hubbell, J. A. Cardiovasc. Pathol. 2003, 12, 295-310.

[2] Peppas, N. A.; Bures, P.; Leobandung, W.; Ichikawa, H. Eur. J. Pharm. Biopharm. 2000, 50, 27-46.

[3] Peppas, N. A.; Khare, A. R. Adv. Drug Delivery Rev. 1993, 11, 1-35.

[4] Kroschwitz, J. I.; Mark, H. F. Encyclopedia of Polymer Science and Technology. 2003, Hoboken, NJ: Wiley-Interscience.

[5] Ruel-Gariepy, E.; Leroux, J. C. Eur. J. Pharm. Biopharm. 2004, 58, 409-426.

[6] Xiong, X. Y.; Tam, K. C.; Gan, L. H. J. App. Polym. Sci. 2006, 100, 4163-4172.

[7] Jeong, B.; Bae, Y. H.; Lee, D. S.; Kim, S. W. Nature 1997, 388, 860-862.

[8] Jeong, B.; Lee, D. S.; Shon, J. I.; Bae, Y. H.; Kim, S. W. J. Polym. Sci., Part A: Polym. Chem. 1999, 37, 751-760.

[9] Hiemstra, C.; Zhong, Z.; Dijkstra, P. J.; Feijen, J. Macromol. Symp. 2005, 224, 119131. 
[10] Bae, S. J.; Suh, J. M.; Sohn, Y. S.; Bae, Y. H.; Kim, S. W.; Jeong, B. Macromolecules 2005, 38, 5260-5265.

[11] Jeong, B.; Bae, Y. H.; Kim, S. W. Colloids Surf. B: Biointerfaces 1999, 16, 185-193.

[12] Park, M. J.; Char, K. Langmuir 2004, 20, 2456-2465.

[13] Shim, M. S.; Lee, H. T.; Shim, W. S.; Park, I.; Lee, H.; Chang, T.; Kim, S. W.; Lee, D. S. J. Biomed. Mater. Res. 2002, 61, 188-196.

[14] Yang, J.; Jia, L.; Hao, Q.; Li, H.; Li, Y.; Fang, Q.; Cao, A. Macromol. Biosci. 2005, 5, 896-903.

[15] Zhong, Z.; Dijkstra, P. J.; Feijen, J.; Kwon, Y. M.; Bae, Y. H.; Kim, S. W. Macromol. Chem. Phys. 2002, 203, 1797-1803.

[16] Lee, S. J.; Han, B. R.; Park, S. Y.; Han, D. K.; Kim, S. C. J. Polym. Sci., Part A: Polym. Chem. 2006, 44, 888-899.

[17] Hiemstra, C.; Zhong, Z.; Li, L. B.; Dijkstra, P. J.; Feijen, J. Biomacromolecules 2006, 7, 2790-2795.

[18] Jiang, Z.; Zhu, Z.; Liu, C.; Hu, Y.; Wu, W.; Jiang, X. Polymer 2008, 49, 5513-5519.

[19] Li, Y.; Kissel, T. Polymer 1998, 39, 4421-4427.

[20] Elbert, D. L.; Hubbell, J. A. Biomacromolecules 2001, 2, 430-441.

[21] Alexandridis, P.; Holzwarth, J. F.; Hatton, T. A. Macromolecules 1994, 27, 2414-2425.

[22] Geppi, M.; Forte, C. J. Magn. Reson. 1999, 137, 177-185.

[23] Lee, D. S.; Shim, M. S.; Kim, S. W.; Lee, H.; Park, I.; Chang, T. Macromol. Rapid Commun. 2001, 22, 587-592.

[24] Hindman, J. C. J. Chem. Phys. 1966, 44, 4582-4592.

[25] Heald, C. R.; Stolnik, S.; Kujawinski, K. S.; de Matteis, C.; Garnett, M. C.; Illum, L.; Davis, S. S.; Purkiss, S. C.; Barlow, R. J.; Gellert, P. R. Langmuir 2002, 18, 3669-3675.

[26] Drumond, W. S.; Mothé, C. G.; Wang, S. H. Polym. Eng. Sci. 2008, 48, 1939-1946.

[27] Riley, T.; Stolnik, S.; Heald, C. R.; Xiong, C. D.; Garnett, M. C.; Illum, L.; Davis, S. S.; Purkiss, S. C.; Barlow, R. J.; Gellert, P. R. Langmuir 2001, 17, 3168-3174.

[28] Yamamoto, Y.; Yasugi, K.; Harada, A.; Nagasaki, Y.; Kataoka, K. J. Controlled Release 2002, 82, 359-371.

[29] Tung, C. Y. M.; Dynes, P. J. J. App. Polym. Sci. 1982, 27, 569-574.

[30] Jeong, B.; Bae, Y. H.; Kim, S. W. Macromolecules 1999, 32, 7064-7069.

[31] Allen, C.; Yu, Y. S.; Maysinger, D.; Eisenberg, A. Bioconjugate Chem. 1998, 9, 564572.

[32] Makino, K.; Arakawa, M.; Kondo, T. Chem. Pharm. Bull. 1985, 33, 1195-1201.

[33] De Jong, S. J.; Arias, E. R.; Rijkers, D. T. S.; van Nostrum, C. F.; Kettenes-van den Bosch, J. J.; Hennink, W. E. Polymer 2001, 42, 2795-2802. 
Chapter 3 


\title{
Chapter 4
}

\section{Stereocomplexed 8-armed PEG-PLA star block copolymer hydrogels}

This chapter has been submitted for publication: Sytze J. Buwalda, ${ }^{\mathrm{a}}$ Lucia Calucci, ${ }^{\mathrm{b}}$ Claudia Forte, ${ }^{\mathrm{b}}$ Pieter J. Dijkstra, ${ }^{a}$ and Jan Feijen ${ }^{\mathrm{a}}$ Macromolecules 2011, submitted.

${ }^{a}$ Department of Polymer Chemistry and Biomaterials, Faculty of Science and Technology, MIRA Institute for Biomedical Technology and Technical Medicine, University of Twente, P.O. Box 217, 7500 AE Enschede, The Netherlands

${ }^{\mathrm{b}}$ Istituto di Chimica dei Composti OrganoMetallici, CNR-Consiglio Nazionale delle Ricerche, Area della Ricerca di Pisa, via G. Moruzzi 1, 56124 Pisa, Italy

\begin{abstract}
Mixing aqueous poly(ethylene glycol)-poly(D-lactide) and poly(ethylene glycol)-poly(L-lactide) star block copolymer solutions ( $>4 \mathrm{w} / \mathrm{v} \%$ ) leads to spontaneous gelation at room temperature due to the formation of poly(lactide) stereocomplexes which act as stable physical crosslinks between micelles and micellar aggregates. The enantiomeric water soluble 8-armed star block copolymers, linked by an amide group (PEG-(NHCO)-(PLA) $)_{8}$ ) or ester group (PEG-(OCO)-(PLA) 8 ) between the PEG core and the PLA blocks were synthesized by the stannous octoate catalyzed ring opening polymerization of D-lactide and L-lactide, respectively, using amine- or hydroxyl terminated 8armed star PEG as an initiator. Stereocomplexed PEG-(NHCO)-(PLA) 8 hydrogels are formed at low polymer concentration and exhibit storage moduli up to $26 \mathrm{kPa}$. Rheology and nuclear magnetic resonance experiments revealed that formation of stereocomplexes is facilitated at higher temperatures, possibly due to rearrangement in the original aggregates thereby exposing more PLA units available for stereocomplexation. The gels formed become temperature irreversible due to the presence of highly stable semi-crystalline stereocomplexed PLA domains. Furthermore, stereocomplexed PEG-(NHCO)-(PLA) 8 hydrogels showed improved in vitro stability and a more sustained release of lysozyme compared to stereocomplexed PEG-(OCO)-(PLA) 8 hydrogels. Because of their good mechanical properties, their potential to be formed in situ and their slow degradation, stereocomplexed PEG-(NHCO)-(PLA) 8 hydrogels are interesting materials for biomedical applications and as controlled drug delivery systems.
\end{abstract}




\subsection{Introduction}

Hydrogels are polymer networks which are able to swell considerably and retain large amounts of water in their swollen structures. ${ }^{1-3}$ Biodegradable poly(ethylene glycol)-poly(lactide) (PEG-PLA) type hydrogels generally exhibit excellent biocompatibility and are accordingly of interest for biomedical applications such as tissue engineering and systems for controlled delivery of biologically active agents. Recently, much effort has been devoted to hydrogels that can be formed in situ under physiological conditions. In situ forming hydrogel systems are injectable fluids that can be introduced into any tissue, organ or body cavity in a minimally invasive manner prior to gelation. ${ }^{4}$ In situ forming hydrogels offer several advantages over systems that have to be formed into their final shape before implantation: there is no need for surgical procedures, their initially flowing nature ensures proper shape adaptation as well as a good fit with surrounding tissue, and biologically active species such as cells or growth factors can be incorporated homogeneously in the hydrogel by simple mixing with the precursor polymer solution. Both chemical and physical crosslinking can be used to create three-dimensional networks in situ. Chemical crosslinking in general provides mechanically stable gels, but care has to be taken of health risks associated with reactive macromonomers and crosslink agents, and practical limitations concerning reaction initiation. In the development of physically crosslinked hydrogels stereocomplexation between enantiomeric PDLA and PLLA blocks in amphiphilic copolymers was shown a powerful tool to provide relatively stable gels.

Early work by the group of Kimura and coworkers revealed that the architecture of stereocomplexed PLA-PEG type block copolymers has a large influence on their solution and gel properties. ${ }^{5,6}$ Whereas stereocomplexed AB diblock and ABA triblock copolymers (with A the PLA block and B the PEG block) show irreversible sol-gel transitions, BAB triblock copolymers appear thermo-reversible. They showed that in general stereocomplex formation cannot be directly related to gel formation. Mixing enantiomeric $\mathrm{AB}$ copolymers affords hydrogels that upon heating to the sol state do not return to the gel state because of formation of a micellar solution in which the micelles have cores composed of highly stable stereocomplexed PLA blocks. The ABA copolymers form gels that upon heating reorganize with the formation of stereocomplexed domains with no gel to sol transition at high or low temperatures. A stereocomplexed gel obtained from a BAB copolymer at high concentration, on the other hand, is thermo-reversible. Thermo-reversibility here is possible because of the presence of bridging molecules between stereocomplexed aggregates. On the contrary, stereocomplexed 8-armed PEG-PLA-PEG triblock copolymers (a branched BAB type copolymer) prepared by coupling carboxylic acid terminated PEG to star shaped PEG-PLA diblock copolymers afforded irreversible gels. ${ }^{7}$ All these results may be ascribed to the formation of a highly phase separated structure with high thermodynamic stability of stereocomplexed domains in micelles and/or aggregates. 
Hydrogels based on stereocomplexation of PLA blocks were mainly studied for their application as a drug delivery system. Li et al. prepared stereocomplexed hydrogels from enantiomeric AB diblock and ABA triblock copolymers. ${ }^{8}$ From these hydrogels, a controlled release of bovine serum albumin was achieved up to $15 \mathrm{~d}$. Zhang et al. investigated the release of the hydrophilic model drug thymopentin from stereocomplexed hydrogels based on PLA-PEG-PLA triblock copolymers. ${ }^{9}$ It was shown that the thymopentin release from stereocomplexed hydrogels was more sustained in comparison with the release from single enantiomer hydrogels. More recently, star shaped block copolymers of PEG and aliphatic polyesters, showing stereocomplex mediated gelation, have been investigated. ${ }^{10}$ It was found that these stereocomplexed PEG-(PLA) 8 star block copolymers gelate faster and form hydrogels with improved mechanical strength as compared to stereocomplexed PLA-PEG-PLA triblock copolymers. It was shown that the release of the relatively small protein lysozyme followed first order kinetics and approximately $90 \%$ was released in $10 \mathrm{~d}^{11}$ The relatively large protein immunoglobulin $\mathrm{G}$ could be released from stereocomplexed hydrogels with nearly zero order kinetics, wherein up to $50 \%$ was released in $16 \mathrm{~d}$.

Previously we found that PEG-(NHCO)-(PLA) 8 star block copolymers, possessing an amide linkage between PEG and PLA, yield hydrogels with good mechanical properties and improved stability towards hydrolytic degradation compared to PEG-(OCO)-(PLA) 8 star block copolymers having an ester linkage between PEG and PLA blocks. ${ }^{12}$ Based on these data, stereocomplexed hydrogels prepared from PEG-(NHCO)-(PLA) 8 were expected to have good mechanical properties and may be well applicable as an injectable controlled drug delivery system. In this paper, the physical, mechanical and degradation properties of stereocomplexed PEG-(NHCO)-(PLA) 8 hydrogels are described together with the release of the model drug lysozyme from these systems.

\subsection{Experimental section}

Materials. Hydroxyl terminated 8-armed poly(ethylene glycol) (PEG- $(\mathrm{OH})_{8}, \mathrm{M}_{\mathrm{n}}$, NMR $=20600$ $\mathrm{g} / \mathrm{mol}$ and $23700 \mathrm{~g} / \mathrm{mol}$ ) was purchased from Jenkem (Allen, Texas, USA) and purified before use by dissolution in dichloromethane and precipitation in cold diethyl ether. The PEG- $(\mathrm{OH})_{8}$ was converted in PEG-(NH$)_{8}$ as described previously. ${ }^{12}$ D-lactide and L-lactide were obtained from Purac (Gorinchem, the Netherlands) and used as received. Tin(II) 2-ethylhexanoate (stannous octoate), methanesulfonyl chloride (mesyl chloride), triethylamine (TEA) and $25 \%$ aqueous ammonia solution were all from Sigma-Aldrich (St Louis, Missouri, USA). Dichloromethane, TEA and toluene were dried over calcium hydride, potassium hydroxide and sodium, respectively, and distilled prior to use.

The 8-armed poly(ethylene glycol)-poly(D-lactide) and poly(ethylene glycol)-poly(L-lactide) star block copolymers (PEG-(NHCO)-(PDLA) 8 and PEG-(NHCO)-(PLLA) $)_{8}$ ) were synthesized by ring opening polymerization of D-lactide and L-lactide, respectively, in toluene at $110{ }^{\circ} \mathrm{C}$. Amine 
terminated 8-armed star PEG $\left(\mathrm{PEG}-\left(\mathrm{NH}_{2}\right)_{8}\right)$ and stannous octoate were used as initiator and catalyst, respectively. This procedure was described in detail previously. ${ }^{12}$

Characterization. ${ }^{1} \mathrm{H}$ NMR $(300 \mathrm{MHz})$ spectra were recorded on a Varian Inova $300 \mathrm{NMR}$ spectrometer. Polymers were dissolved in $\mathrm{CDCl}_{3}$ at a concentration of $15 \mathrm{mg} / \mathrm{ml}$. Thermal properties of polymers were determined using differential scanning calorimetry (DSC). Heating and cooling rates of $20{ }^{\circ} \mathrm{C} / \mathrm{min}$ were applied. Samples were heated from 25 to $200{ }^{\circ} \mathrm{C}$, kept at $200{ }^{\circ} \mathrm{C}$ for $1 \mathrm{~min}$, cooled to $-50{ }^{\circ} \mathrm{C}$, kept at $-50{ }^{\circ} \mathrm{C}$ for $1 \mathrm{~min}$, and finally heated to $200{ }^{\circ} \mathrm{C}$. Crystallization temperatures $\left(\mathrm{T}_{\mathrm{c}}\right)$ and corresponding enthalpies $\left(\Delta \mathrm{H}_{\mathrm{c}}\right)$ were obtained from the cooling scan, while melting temperatures $\left(\mathrm{T}_{\mathrm{m}}\right)$ and corresponding enthalpies $\left(\Delta \mathrm{H}_{\mathrm{m}}\right)$ were obtained from the second heating scan.

Aqueous solution properties. The critical association concentration (CAC) values of aqueous solutions of the PEG-PLA star block copolymers were determined by a fluorescence technique using pyrene as a probe. ${ }^{13}$ In a hydrophobic environment such as a micellar core, pyrene molecules exhibit a much stronger fluorescence than in a hydrophilic environment, leading to a redshift of their excitation spectrum. The onset of this redshift relates to the CAC. Mixed enantiomer solutions (D/L ratio 1/1) of star block copolymers in the concentration range of 0.0025 to $2 \mathrm{w} / \mathrm{v} \%$ were prepared using distilled water. An aqueous solution of pyrene was prepared at a concentration of $1.2 \cdot 10^{-6} \mathrm{M}$. Approximately $100 \mu \mathrm{l}$ of polymer solution was added to a well in a 96-well culture plate, followed by addition of $100 \mu \mathrm{l}$ of the pyrene solution. Fluorescence spectra were obtained with a Tecan Safire microplate reader at room temperature. The detection wavelength was set at 383 or $390 \mathrm{~nm}$. The fluorescence intensity ratio $\left(\mathrm{I}_{326} / \mathrm{I}_{311}\right.$ or $\left.\mathrm{I}_{328} / \mathrm{I}_{314}\right)$ of pyrene excitation spectra was plotted against the logarithm of the polymer concentration and the intercept of the extrapolated straight lines was taken as the CAC.

Dynamic light scattering (DLS) of dilute solutions $(0.5 \mathrm{w} / \mathrm{v} \%)$ of mixed enantiomer PEG-PLA star block copolymers ( $\mathrm{D} / \mathrm{L}$ ratio $1 / 1)$ in water was performed to determine aggregate sizes. Experiments were carried out at $25{ }^{\circ} \mathrm{C}$ using a Malvern Nano ZS, a laser wavelength of $633 \mathrm{~nm}$ and a scattering angle of $173^{\circ}$.

Gel properties. The vial tilting method was used to determine the critical gel concentration (CGC) at room temperature as well as the gel to sol transition of PEG-PLA star block copolymers. Polymers were dissolved in distilled water in tightly capped glass vials. The reversible gel to sol transition of polymer solutions containing the D- and L-enantiomer in equal amounts was studied in the range of 2 to $80{ }^{\circ} \mathrm{C}$ with temperature increments of $2{ }^{\circ} \mathrm{C}$. At each temperature, the sample was 
allowed to equilibrate for $10 \mathrm{~min}$. If there was no flow after tilting the vials $90^{\circ}$ for $1 \mathrm{~min}$, the sample was regarded as a gel; otherwise it was regarded as a sol.

${ }^{1} \mathrm{H}$ NMR experiments on aqueous solutions and gels were carried out on a Bruker AMX-300 WB spectrometer working at $300.13 \mathrm{MHz}$ for proton and $46.04 \mathrm{MHz}$ for deuterium, using a $5 \mathrm{~mm}$ probe head. The samples, containing 5 and $10 \mathrm{w} / \mathrm{v} \%$ of PEG-(NHCO)-(PDLA 11$)_{8}$, PEG-(NHCO)$\left(\mathrm{PLLA}_{11}\right)_{8}$, or $1: 1$ mixtures of the two copolymers, were prepared by dissolving the appropriate amounts of copolymer in $\mathrm{D}_{2} \mathrm{O}(99.98 \% \mathrm{D}$, Eurisotop); repeated heating cycles were necessary to obtain homogeneous samples. ${ }^{1} \mathrm{H}$ NMR spectra were recorded on all samples at 25, 40, 55, and 70 ${ }^{\circ} \mathrm{C}$ using a $90^{\circ}$ pulse of $5.7 \mu$ s and a recycle delay ranging from 5 to $30 \mathrm{~s}$, depending on sample and temperature, and acquiring 64 scans. Relative peak intensities within each spectrum were determined by integration of the peaks and were then calibrated using 3-(trimethylsilyl)-1propanesulfonic acid sodium salt (DSS) at known concentration as a standard. DSS was also used as reference for the ${ }^{1} \mathrm{H}$ chemical shifts. Spin-lattice relaxation times $\left(\mathrm{T}_{1}\right)$ of polymer protons were determined in the $10 \mathrm{w} / \mathrm{v} \%$ samples at $25,40,55$ and $70{ }^{\circ} \mathrm{C}$, using the inversion recovery pulse sequence with a recycle delay ranging from 5 to $30 \mathrm{~s}$, depending on sample and temperature, and acquiring 32 scans. The baseline corrected areas of the peaks $(I(t))$ at different values of the characteristic delay of the pulse sequence were measured and $T_{1}$ values were determined by fitting the inversion recovery curve using the equation:

$$
\mathrm{I}(\mathrm{t})=\mathrm{I}_{\infty}\left(1-\alpha \exp \left(-\mathrm{t} / \mathrm{T}_{1}\right)\right)
$$

where $I_{\infty}$ is the signal intensity at time $\infty$ and $\alpha$ is a parameter accounting for non-complete magnetization inversion. ${ }^{14}{ }^{2} \mathrm{H}$ inversion recovery experiments were also performed on $10 \mathrm{w} / \mathrm{v} \%$ samples at $25,40,55$ and $70{ }^{\circ} \mathrm{C}$, using a $9.5 \mu$ s $90^{\circ}$ pulse, a recycle delay of $8 \mathrm{~s}$, and acquiring 8 scans. The area of the baseline corrected $\mathrm{D}_{2} \mathrm{O}$ peak was measured in the spectra and used to determine water ${ }^{2} \mathrm{H}_{1}$ values through equation (1). For all experiments the temperature was controlled within $0.1^{\circ} \mathrm{C}$.

Oscillatory rheology experiments were performed to determine the mechanical properties of stereocomplexed hydrogels. The storage $\left(G^{\prime}\right)$ and loss $\left(G^{\prime \prime}\right)$ modulus of hydrogels were monitored at temperatures ranging from 20 to $60{ }^{\circ} \mathrm{C}$ on an Anton-Paar Physica MCR 301 rheometer. To determine the kinetics of gel formation, separate solutions of D-enantiomer and L-enantiomer (D/L ratio 1/1) were mixed, homogenized and quickly applied to the rheometer. Experiments were performed using a flat plate measuring geometry (diameter $25 \mathrm{~mm}$, gap $0.3 \mathrm{~mm}$ ) utilizing a strain of $0.5 \%$ and a frequency of $1 \mathrm{~Hz}$. To prevent water evaporation, a solvent trap was placed over the geometry. To confirm that the strain is within the viscoelastic regime, an amplitude sweep from 0.1 to $10 \%$ was performed at $1 \mathrm{~Hz}$. 
Release experiments. Hydrogels were loaded with lysozyme by dissolving the lyophilized protein in macromonomer PBS solutions prior to gelation. The initial protein concentration was 10 wt $\%$ relative to the total polymer mass. For the in vitro release of lysozyme, $3 \mathrm{ml}$ of PBS was placed on top of $0.5 \mathrm{ml}$ of hydrogel and the vials were kept at $37^{\circ} \mathrm{C}$. To prevent bacterial growth, $0.02 \mathrm{w} / \mathrm{v} \% \mathrm{NaN}_{3}$ was added to the buffer solution. At regular time intervals, $50 \mu \mathrm{l}$ of supernatant was removed and replaced by fresh buffer. The concentration of lysozyme in the release samples was determined using the $\mathrm{BCA} \AA$ Protein Assay. Briefly, $25 \mu \mathrm{l}$ of release sample was mixed with $200 \mu \mathrm{l}$ of working reagent (BCA reagent A : BCA reagent $\mathrm{B}, 50: 1 \mathrm{v} / \mathrm{v}$ ) in a 96-wells plate. The plate was incubated at $37{ }^{\circ} \mathrm{C}$ for $30 \mathrm{~min}$ and the absorbance was read at $562 \mathrm{~nm}$ using a Tecan Safire microplate reader. A calibration curve was constructed using protein solutions of known concentration. For selected samples, the activity of the released lysozyme was determined using an assay based on Micrococcus lysodeikticus. ${ }^{15}$ Lysis of the outer cell membranes by lysozyme results in solubilization of the bacteria and, as a consequence, a decrease in light scattering. Briefly, $20 \mu 1$ of release sample was added to $2.6 \mathrm{ml}$ of a bacterial suspension $(0.2 \mathrm{mg} / \mathrm{ml}$ in PBS $)$ and the decrease in absorbance at $450 \mathrm{~nm}$ was measured for $1 \mathrm{~h}$. The enzymatic activity of the lysozyme in the release sample was determined by comparing the decrease in absorbance with that in a bacterial suspension containing freshly prepared lysozyme solution.

Degradation. Gravimetric degradation/dissolution experiments were performed to determine the stability of the stereocomplexed hydrogels. The experiments were conducted for unloaded hydrogels and for hydrogels loaded with lysozyme (10 wt \% relative to the total polymer mass). On top of $0.5 \mathrm{ml}$ of hydrogel $3 \mathrm{ml}$ of PBS was placed and the samples were kept at $37{ }^{\circ} \mathrm{C}$. At regular time intervals, $2.5 \mathrm{ml}$ of the supernatant was removed and replaced by fresh buffer. The supernatant samples, taken at various time points, were freeze-dried and the mass of solubilized polymer was determined gravimetrically by subtracting the mass of the PBS salts from the mass of the sample. Degradation/dissolution experiments were performed in duplo. The structure of the remaining polymers in hydrogel samples was analyzed by ${ }^{1} \mathrm{H}$ NMR spectroscopy. After freeze-drying of a sample, polymers were dissolved in a minimal amount of dichloromethane and the solution was precipitated in an excess of a mixture of cold diethyl ether and methanol $(20 / 1 \mathrm{v} / \mathrm{v})$. The precipitate was collected by filtration, dried under a nitrogen flow and analyzed by ${ }^{1} \mathrm{H}$ NMR. Oscillatory rheology was used to investigate the mechanical properties of the hydrogels upon degradation by determining $\mathrm{G}^{\prime}$ and $\mathrm{G}$ ', of hydrogels as described above. 


\subsection{Results and discussion}

Synthesis and characterization of PEG-PLA star block copolymers. Enantiomeric PEG(NHCO)-(PDLA) 8 and PEG-(NHCO)-(PLLA) 8 star block copolymers were prepared by the stannous octoate catalyzed ring opening polymerization of D-lactide and L-lactide, respectively, initiated by PEG-( $\left.\mathrm{NH}_{2}\right)_{8}\left(\mathrm{M}_{\mathrm{n}, \mathrm{NMR}}=23700 \mathrm{~g} / \mathrm{mol}\right)$ (Figure 1). ${ }^{12}$ The 8-armed PEG-(OCO)-(PDLA) 8 and PEG-(OCO)-(PLLA $)_{8}$ star block copolymers were prepared analogously, using PEG-(OH) 8 $\left(\mathrm{M}_{\mathrm{n}, \mathrm{NMR}}=20600 \mathrm{~g} / \mathrm{mol}\right)$ as the initiator (Figure 1). ${ }^{10}$

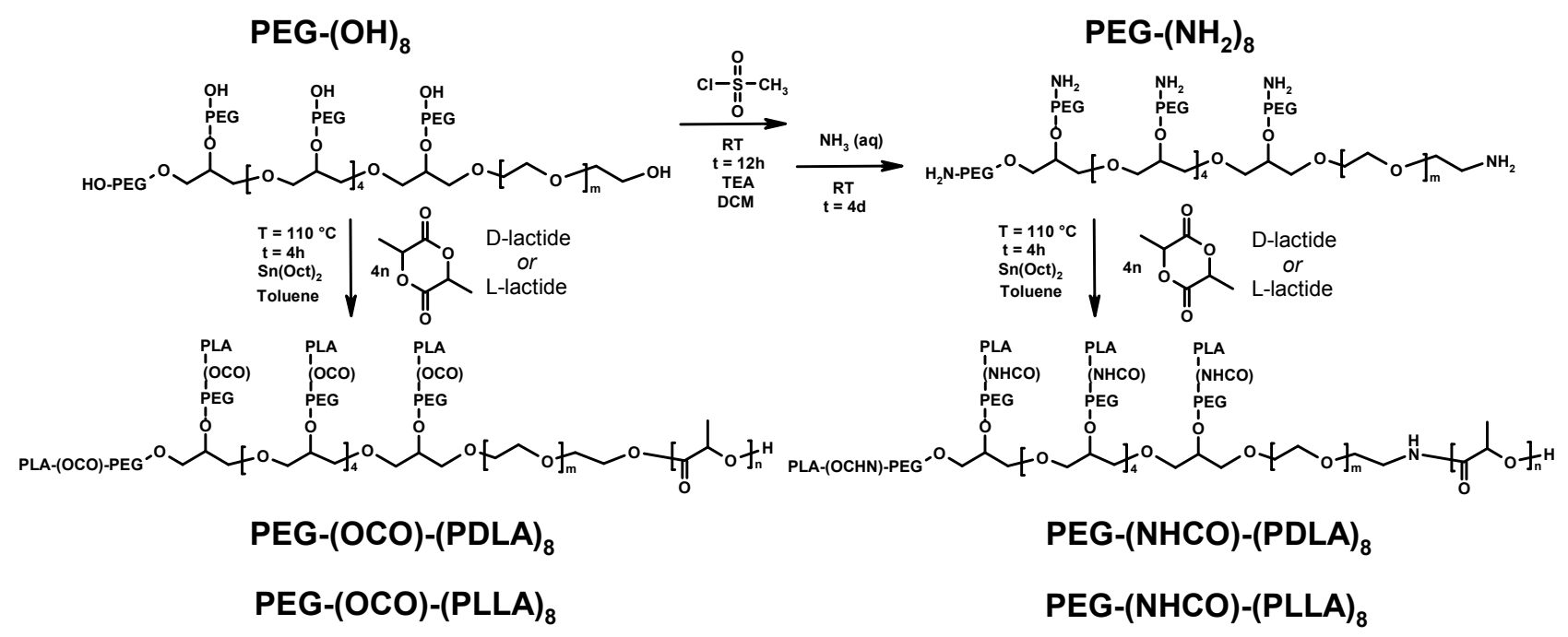

Figure 1. Synthesis scheme for the preparation of PEG-(NHCO)-(PLA) ${ }_{8}$ and PEG-(OCO)-(PLA) 8 star block copolymers.

Star block copolymers with a degree of polymerization (DP) of 11 or 13 lactyl units per PLA arm were selected (Table 1), because higher DPs of the PLA arms led to a decreased aqueous solubility whereas lower DPs would hamper stereocomplex formation. ${ }^{16}$ The aqueous solubility of the 8armed PEG-(NHCO)-(PLA) 8 block copolymers appeared better than that of the 8-armed PEG(OCO)-(PLA) 8 block copolymers. Whereas the former gave transparent solutions and gels at room temperature up to high concentrations $(<20 \mathrm{w} / \mathrm{v} \%)$ the latter type copolymer solutions and gels always appeared opaque. 
Table 1. Molecular weights and thermal properties of PEG initiators and PEG-PLA star block copolymers.

\begin{tabular}{|c|c|c|c|c|c|c|c|c|}
\hline \multirow{2}{*}{ Polymer } & \multicolumn{2}{|c|}{${ }^{1} \mathrm{H}$ NMR } & \multirow{2}{*}{$\begin{array}{c}\mathrm{T}_{\mathrm{m}} \\
\left({ }^{\circ} \mathrm{C}\right)\end{array}$} & \multirow{2}{*}{$\begin{array}{l}\Delta \mathrm{H}_{\mathrm{m}} \\
(\mathrm{J} / \mathrm{g})\end{array}$} & \multirow{2}{*}{$\begin{array}{c}\mathrm{T}_{\mathrm{c}} \\
\left({ }^{\circ} \mathrm{C}\right)\end{array}$} & \multirow{2}{*}{$\begin{array}{l}\Delta \mathrm{H}_{\mathrm{c}} \\
(\mathrm{J} / \mathrm{g})\end{array}$} & \multirow{2}{*}{$\frac{\text { CAC }}{(w / v}$} & \multirow{2}{*}{$\begin{array}{l}\text { CGC } \\
\%)\end{array}$} \\
\hline & $\mathrm{DP}^{\mathrm{a}}$ & $\mathrm{M}_{\mathrm{n}}^{\mathrm{b}}$ & & & & & & \\
\hline PEG-(NH $)_{8}$ & - & 23.7 & 53 & 126 & 28 & 126 & - & - \\
\hline $\begin{array}{c}\text { PEG-(NHCO)-(PDLA } 11)_{8} \\
+ \\
\text { PEG-(NHCO)-(PLLA } 11)_{8}\end{array}$ & 12.0 & 30.6 & 41 & 56 & 13 & 57 & 0.20 & 5 \\
\hline $\begin{array}{c}\text { PEG-(NHCO)-(PDLA } 13)_{8} \\
+ \\
\text { PEG-(NHCO)-(PLLA } 13)_{8}\end{array}$ & 13.3 & 31.4 & 40 & 55 & 3 & 55 & 0.10 & 4 \\
\hline $\begin{array}{l}\text { PEG-(OH) } \\
8\end{array}$ & - & 20.6 & 54 & 141 & 31 & 141 & - & - \\
\hline $\begin{array}{c}\text { PEG-(OCO)-(PDLA } 13)_{8} \\
+ \\
\text { PEG-(OCO)-(PLLA } 13)_{8}\end{array}$ & 13.3 & 28.3 & 40 & 56 & 6 & 56 & 0.06 & 14 \\
\hline
\end{tabular}

${ }^{a}$ Degree of polymerization of the PLA blocks, expressed in lactyl units per arm.

${ }^{\mathrm{b}}$ Expressed in $\mathrm{kg} / \mathrm{mol}$.

The thermal properties of enantiomeric mixtures of the block copolymers as determined with differential scanning calorimetry (DSC) revealed major transitions in both second heating and cooling scans, corresponding to melting and crystallization of the PEG domains, respectively (Table 1). All enantiomeric mixtures of the PEG-PLA star block copolymers exhibit lower melting transitions and accompanying enthalpies than their PEG precursors. Apparently, the crystallization of PEG is hampered by the presence of PLA blocks. Only in case of the stereocomplexed PEG(NHCO)-(PLA) 8 copolymers, a small exothermic peak was observed at $\sim 165{ }^{\circ} \mathrm{C}$. This transition, which can be attributed to melting of stereocomplex crystals, was not observed for stereocomplexed PEG-(OCO)-(PLA) 8 .

Gelation behavior. The structure of 8 -armed PEG-(PLA) 8 block copolymers resembles to a certain extent ABA type triblock copolymers with PLA end blocks and a central PEG block. The single enantiomer 8-armed PEG-(NHCO)-(PLA $)_{8}$ and PEG-(OCO)-(PLA 13$)_{8}$ block copolymers with short PLA blocks (DP 11-15 lactyl units) show gel to sol phase transitions at concentrations ranging from 7 to $20 \mathrm{w} / \mathrm{v} \%{ }^{12}$ To study the gel to sol transitions of the stereocomplexed 8 -armed block copolymers enantiomeric polymer solutions were prepared, combined and the gel to sol phase transitions were determined with the vial tilting method in the range of 2 to $80{ }^{\circ} \mathrm{C}$ (Figure 2 ). 


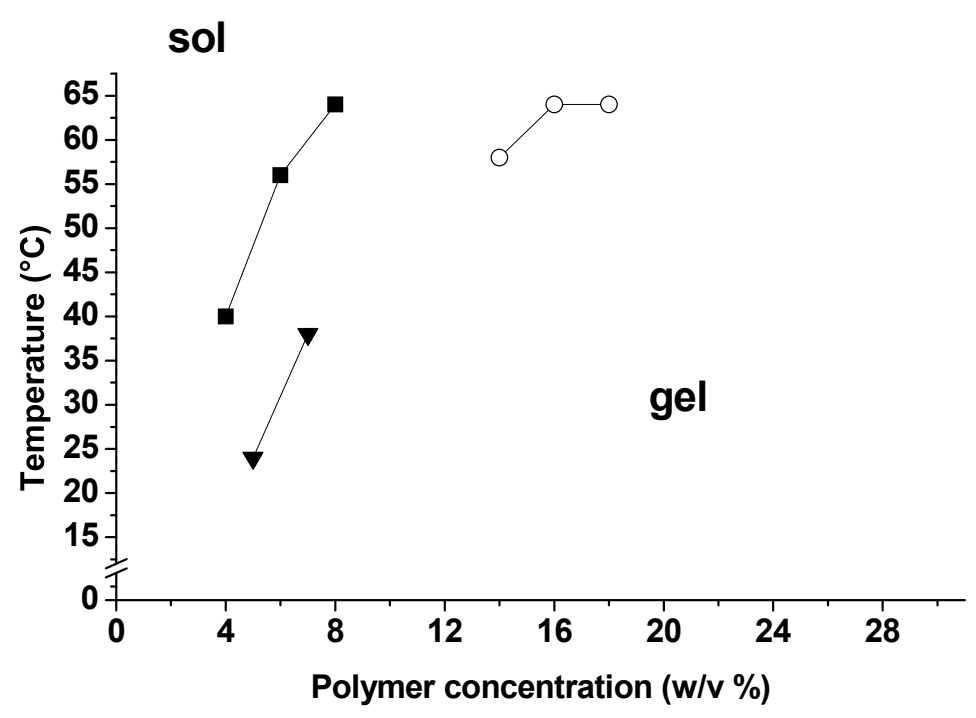

Figure 2. Gel to sol transition temperatures of stereocomplexed PEG-PLA hydrogels (D:L=1:1).

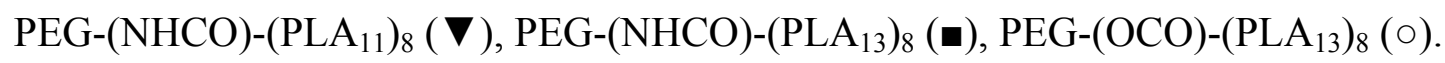

The mixed enantiomer solutions of PEG-(NHCO)-( PLA $\left._{n}\right)_{8}$ formed a hydrogel almost instantaneously at low concentrations whereas the gelation times for mixed enantiomer solutions of PEG-(OCO)-( $\left.\mathrm{PLA}_{13}\right)_{8}$ were approximately $30 \mathrm{~min}$ at higher concentrations. Upon heating, a gel to sol transition could be observed but it was limited to a narrow concentration range of $\sim 4-8 \mathrm{w} / \mathrm{v} \%$ for stereocomplexed PEG-(NHCO)-(PLA $)_{8}$ and 14-18 w/v \% for stereocomplexed PEG-(OCO)$\left(\mathrm{PLA}_{13}\right)_{8}$. At higher concentrations no gel to sol transition was observed up to the boiling temperature of water, whereas at lower concentrations no gels were formed. Repeating the heating and cooling cycle revealed a progressive increase in temperature of the gel to sol phase transition.

Combining all the above reported results some general conclusions can be drawn: i) stereocomplexation leads to gel formation at lower concentrations and gel to sol transitions at higher temperatures compared to single enantiomer 8-armed PEG-PLA star block copolymers, ii) an increase in polymer concentration shifts the gel to sol transition to higher temperatures as a result of an increased number of physical crosslinks, iii) increasing the DP of the hydrophobic block shifts the gel to sol transition to higher temperatures, which is likely a result of the more facile formation of stereocomplexed crystals at higher DP, iv) replacing the ester group with an amide group as linking unit between the hydrophilic PEG and hydrophobic PLA block gives a shift of the boundary curve to lower concentrations indicating an even more efficient interaction between PLA chains, v) hydrogels formed from stereocomplexed 8-armed block copolymers become irreversible after repeated heating and cooling cycles due to the slow formation of stereocomplexed crystals.

Aggregate size and size distribution in dilute aqueous solution. The critical association concentration (CAC) values of the single enantiomers of the 8 -armed block copolymers $(0.20$ to $0.50 \mathrm{w} / \mathrm{v} \%$ ) are in general somewhat higher than the values of the corresponding stereocomplexed 
PEG-(PLA) 8 (Table 1). The CAC value decreases at higher PLA block length as a result of increased hydrophobic interactions. The enhanced tendency of these stereocomplexed block copolymers to form aggregates, compared to single enantiomer copolymers, can be ascribed to the stronger interaction between PLLA and PDLA blocks. ${ }^{17}$ Dynamic light scattering (DLS) experiments revealed that two size distributions are present in dilute aqueous solutions of mixed enantiomers (Figure 3). The results show that above the CAC these materials form small aggregates with diameters ranging from $20-50 \mathrm{~nm}$, indicative of micellar type structures, as well as larger aggregates with a diameter between 100 and $400 \mathrm{~nm}$. For all stereocomplexed materials the size distribution is shifted to larger aggregates compared to the single enantiomers, ${ }^{12}$ similar to previous observations on stereocomplexing systems. ${ }^{10,17}$

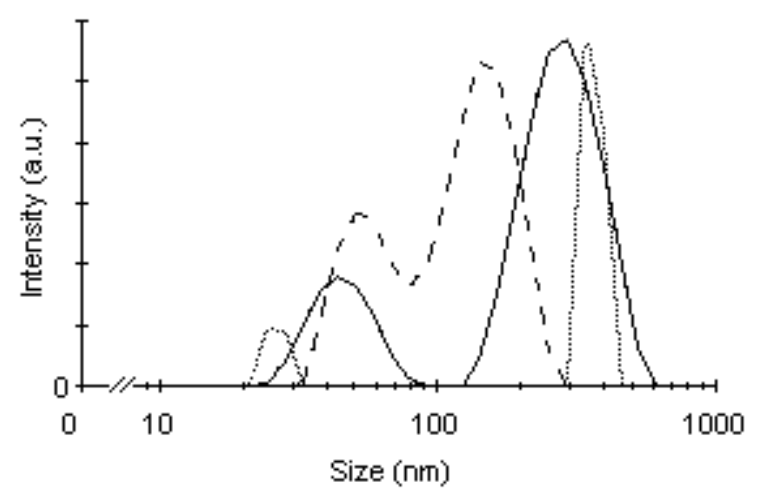

Figure 3. Aggregate size distributions in $0.5 \mathrm{w} / \mathrm{v} \%$ aqueous solutions containing mixed enantiomers at room temperature. PEG-(NHCO)-(PLA 11$)_{8}$ (solid line), PEG-(NHCO)-(PLA 13$)_{8}$ (dashed line), PEG-(OCO)-(PLA $\left.{ }_{13}\right)_{8}$ (dotted line).

NMR spectroscopy on aqueous solutions and hydrogels. ${ }^{1} \mathrm{H}$ NMR spectroscopy was employed to investigate the aggregation behavior of stereocomplexes in water in comparison with that of the single enantiomer. To this aim ${ }^{1} \mathrm{H}$ NMR spectra were recorded at different temperatures (i.e. 25, 40, 55 , and $70{ }^{\circ} \mathrm{C}$ ) on $5 \mathrm{w} / \mathrm{v} \%$ and $10 \mathrm{w} / \mathrm{v} \%$ samples of PEG-(NHCO)-(PDLA 11$)_{8}$, PEG-(NHCO)$\left(\mathrm{PLLA}_{11}\right)_{8}$ and $1: 1$ mixtures of the two copolymers in $\mathrm{D}_{2} \mathrm{O}$. It must be emphasized that the aggregation behavior was investigated at concentrations lower than the critical gel concentration (CGC) for the single enantiomers, and equal to or higher than the CGC for the $5 \mathrm{w} / \mathrm{v} \%$ and the 10 w/v \% copolymer mixtures, respectively (Table 1). As previously reported, ${ }^{18}$ the spectra of the single enantiomers in water show a peak ascribable to the PEG methylene protons at $3.698 \mathrm{ppm}$, several signals ascribable to the PLA methyl protons in the spectral region between 1.3 and $1.6 \mathrm{ppm}$ and the signals arising from the PLA methine protons in the regions 4.2-4.5 and 5.0-5.3 ppm, as well as the signal of HDO at approximately $4.8 \mathrm{ppm}$. All the PEG methylene protons were detected at all temperatures, as inferred from comparison with an external standard, whereas the intensities of the PLA peaks in the spectra were lower than expected on the basis of stoichiometry with respect 
to the methylene peaks of the PEG chain. In particular, the fraction of PLA units detected at $25{ }^{\circ} \mathrm{C}$ for both PEG-(NHCO)-(PDLA 11$)_{8}$ and PEG-(NHCO)-(PLLA 11$)_{8}$ was $27 \pm 2 \%$ and $23 \pm 2 \%$ for the $5 \mathrm{w} / \mathrm{v} \%$ and $10 \mathrm{w} / \mathrm{v} \%$ samples, respectively. On increasing the temperature up to $70{ }^{\circ} \mathrm{C}$, this fraction increased up to $81 \pm 2 \%$ and $73 \pm 2 \%$ for the $5 \mathrm{w} / \mathrm{v} \%$ and $10 \mathrm{w} / \mathrm{v} \%$ samples, respectively. The variation of the PLA methyl signals with temperature is shown for the $10 \mathrm{w} / \mathrm{v} \%$ PEG-(NHCO)-(PLLA $\left.{ }_{11}\right)_{8}$ sample in Figure 4a. This behavior is due to the formation of solid-like aggregates of PLA chains which prevents the observation of all PLA protons in solution state NMR experiments. The PLA proton signals detected at room temperature were attributed, on the basis of their chemical shift and trend with temperature, to chains dangling in water and to chains in unimolecular or flower-like micelles; the increase in intensity with temperature could be ascribed to a progressive increase in PLA chain mobility within intermicellar aggregates, which were also observed in DLS experiments (vide supra). ${ }^{18}$

(a)

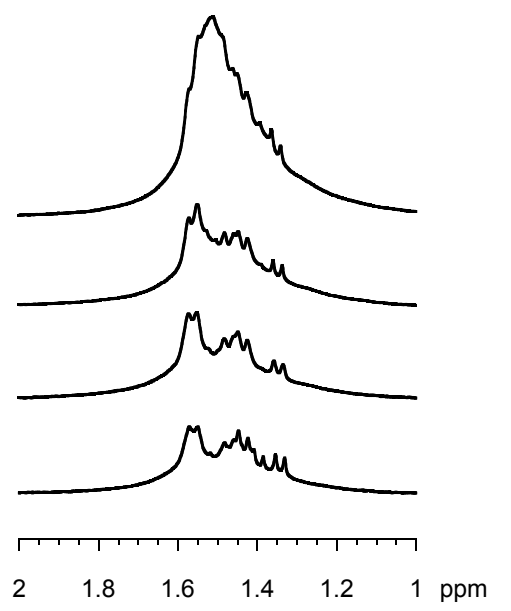

(b)

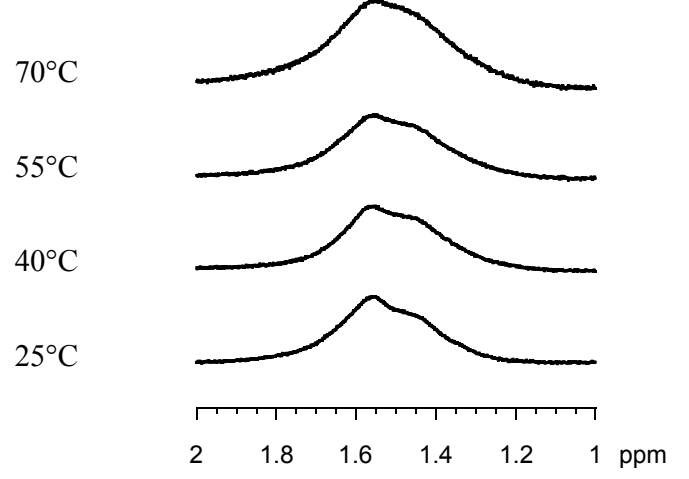

Figure 4. ${ }^{1} \mathrm{H}$ NMR methyl spectral region of $10 \mathrm{w} / \mathrm{v} \%$ samples of PEG-(NHCO)-(PLLA 11$)_{8}(\mathrm{a})$ and PEG-(NHCO)-(PDLA 11$)_{8}+$ PEG-(NHCO)-(PLLA $\left.{ }_{11}\right)_{8}(b)$ in $\mathrm{D}_{2} \mathrm{O}$ at the indicated temperatures.

At $25{ }^{\circ} \mathrm{C}$ the ${ }^{1} \mathrm{H}$ NMR spectrum of the $5 \mathrm{w} / \mathrm{v} \%$ sample of the $1: 1 \mathrm{PEG}-(\mathrm{NHCO})-\left(\mathrm{PDLA} \mathrm{A}_{11}\right)_{8} /$ PEG-(NHCO)-(PLLA $\left.{ }_{11}\right)_{8}$ mixture in $\mathrm{D}_{2} \mathrm{O}$ was very similar to those of the single enantiomer copolymers, whereas that of the $10 \mathrm{w} / \mathrm{v} \%$ sample was characterized by broader lines due to the formation of the gel phase. In the gel phase the PLA methyl and methine signals were both roughly represented by two peaks at approximately 1.58 and $1.45 \mathrm{ppm}$, and 5.26 and $5.10 \mathrm{ppm}$, respectively, while a peak at $3.70 \mathrm{ppm}$ was observed for PEG methylene protons. These chemical shifts are similar to those previously reported for the single enantiomers at concentrations above the CGC. ${ }^{12}$ Also for the enantiomeric mixtures all the PEG methylene protons were detected at all temperatures, whereas the fraction of detected PLA protons was always lower than that expected on the basis of stoichiometry with respect to the PEG methylene protons. However, differently from the case of the single enantiomers at concentrations below (here investigated) and above ${ }^{12,18}$ the 
CGC, the increase of the amount of PLA protons detected on increasing the temperature was quite small (see Figure 4b). In fact, the fraction of observed PLA units increased from $20 \pm 2$ to only $28 \pm$ $2 \%$ on going from $25{ }^{\circ} \mathrm{C}$ to $70{ }^{\circ} \mathrm{C}$ for both the 5 and $10 \mathrm{w} / \mathrm{v} \%$ samples, indicating that in this case the intermolecular PLA aggregates remain solid-like also at $70{ }^{\circ} \mathrm{C}$. The different behavior observed for single enantiomers and stereocomplex can be ascribed to the different state of PLA chains in the hydrophobic aggregates. For single enantiomers, the PLA domains are mainly amorphous, with a glass transition in the temperature range investigated, ${ }^{19}$ whereas for the stereocomplex, also crystalline PLA domains are present, with a melting temperature of approximately $165{ }^{\circ} \mathrm{C}$.

Importantly, since no spectral differences were detected for PEG methylene protons between the single enantiomer samples and the mixtures, the formation of a stronger network does not seem to influence the dynamics of the PEG chains. Moreover, the fact that the PEG signal did not show any temperature dependence is a strong indication that PEG entanglements or deswelling do not occur at least in the temperature range investigated. The trends with temperature $\left(25-70{ }^{\circ} \mathrm{C}\right)$ of the PEG ${ }^{1} \mathrm{H}$ spin-lattice relaxation times $\left(\mathrm{T}_{1}\right)$ measured on the $10 \mathrm{w} / \mathrm{v} \%$ samples of the single enantiomers and of the 1:1 mixture in $\mathrm{D}_{2} \mathrm{O}$ confirmed that the PEG chains are in all cases well swollen in water and quite mobile. In fact, essentially the same $T_{1}$ values were determined for PEG methylene protons in all samples at the different temperatures ( $\mathrm{T}_{1}$ ranging from $550 \pm 10 \mathrm{~ms}$ to $1350 \pm 10 \mathrm{~ms}$ on going from $25^{\circ} \mathrm{C}$ to $70{ }^{\circ} \mathrm{C}$ ), and these values were quite similar to those reported for pure PEG in water, ${ }^{20,21}$ where the PEG chains undergo fast motions. Finally, water ${ }^{2} \mathrm{H} \mathrm{T}_{1}$ values measured for the same samples in the $25-70{ }^{\circ} \mathrm{C}$ temperature range $\left(\mathrm{T}_{1}\right.$ ranging from $350 \pm 10 \mathrm{~ms}$ to $990 \pm 10 \mathrm{~ms}$ on heating) were indicative of a bulk-like behavior of water, ${ }^{18}$ irrespective of the network strength.

Rheology. The storage $\left(\mathrm{G}^{\prime}\right)$ and loss $\left(\mathrm{G}^{\prime \prime}\right)$ moduli of stereocomplexed PEG-(NHCO)-(PLA 11$)_{8}$ and PEG-(OCO)-(PLA $\left.)_{13}\right)_{8}$ hydrogels were determined with oscillatory rheology measurements at $20{ }^{\circ} \mathrm{C}$. For all samples, G' exceeded G', confirming that the systems were in the gel state. ${ }^{22}$ The dependence of $G^{\prime}$ on polymer concentration (Figure 5) was almost linear, but is shifted to lower concentrations for the stereocomplexed PEG-(NHCO)-(PLA $\left.\mathrm{PL}_{11}\right)_{8}$. 


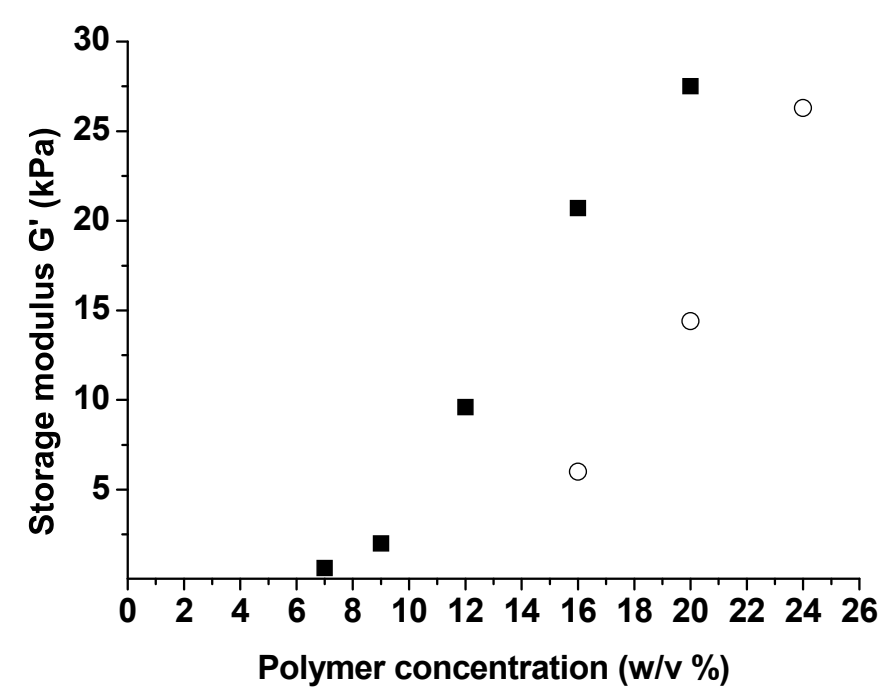

Figure 5. Storage modulus $\left(\mathrm{G}^{\prime}\right)$ as a function of the polymer concentration at $20{ }^{\circ} \mathrm{C}$ for stereocomplexed PEG-PLA hydrogels. PEG-(NHCO)-(PLA $\left.)_{11}\right)_{8}(\boldsymbol{\varpi})$, PEG-(OCO)-(PLA $\left.)_{13}\right)_{8}(\circ)$.

It is apparent from Figure 5 that at the same concentration hydrogels based on a racemic mixture of PEG-(NHCO)-(PLA $\left.)_{11}\right)_{8}$ exhibit a much higher gel stiffness than hydrogels based on a racemic mixture of PEG-(OCO)-(PLA $\left.{ }_{13}\right)_{8}$. As an example, a $16 \mathrm{w} / \mathrm{v} \%$ stereocomplexed PEG-(OCO)$\left(\mathrm{PLA}_{13}\right)_{8}$ hydrogel has a storage modulus of $6 \mathrm{kPa}$, whereas a $16 \mathrm{w} / \mathrm{v} \%$ stereocomplexed PEG(NHCO)-(PLA $\left.)_{11}\right)_{8}$ hydrogel has a storage modulus of $21 \mathrm{kPa}$. These results show that replacing an ester bond with an amide bond in the PEG-(PLA) 8 results in a more efficient crosslinking, a trend similar to the difference in CGC between the two systems. In comparison with single enantiomer hydrogels at similar polymer concentrations, ${ }^{12}$ stereocomplexed hydrogels exhibit a 10-15 $\mathrm{kPa}$ higher gel stiffness, suggesting a higher effective crosslink density. The increase in G' with concentration can be ascribed to the formation of a more densely physically crosslinked network.

Gel formation kinetics were studied by monitoring the storage modulus ( $\left.\mathrm{G}^{\prime}\right)$ and loss modulus $\left(G^{\prime \prime}\right)$ in time after mixing solutions of macromonomers with opposite chirality. When $6 \mathrm{w} / \mathrm{v} \%$ PEG-(OCO)-(PDLA 13$)_{8}$ and PEG-(OCO)-(PLLA 13$)_{8}$ solutions were mixed, the crossover point of G' and G', which can be regarded as the gel point, was observed after 33 min (Figure 6A and 6B). The storage modulus levels off after approximately $48 \mathrm{~h}$, showing that gelation is complete. When 6 w/v \% PEG-(NHCO)-(PDLA 13$)_{8}$ and PEG-(NHCO)-(PLLA 13$)_{8}$ solutions were mixed, G' crossed G', within 1 min (Figure 6C and 6D). Importantly, the stereocomplexed PEG-(NHCO)-(PLA 13$)_{8}$ hydrogels show much lower damping factors ( $\left.\tan \delta=\mathrm{G}^{\prime}, \mathrm{G}^{\prime}\right)$ compared to the stereocomplexed PEG-(OCO)-( $\left.\mathrm{PLA}_{13}\right)_{8}$ hydrogels. This indicates a more elastic, i.e. gel-like, behavior of the stereocomplexed PEG-(NHCO)-( PLA $\left._{13}\right)_{8}$ hydrogels. ${ }^{23}$ The faster gelation kinetics and lower damping factor of stereocomplexed PEG-(NHCO)-(PLA $\left.{ }_{13}\right)_{8}$ hydrogels (Figure 6) and their higher storage moduli (Figure 5) may be explained by the conformational rigidity of the amide groups, which may result in better phase separation between the hydrophilic and hydrophobic blocks. This 
enhanced phase separation allows for easier crystallization of PLA stereocomplexes as indicated by the DSC results and thereby a better physically crosslinked network. For all samples G' and G', showed little variation when an amplitude sweep from 0.1 to $10 \%$ was performed at the end of each rheological experiment, indicating that a strain of $0.5 \%$ is within the linear viscoelastic range of the hydrogels. $^{24}$
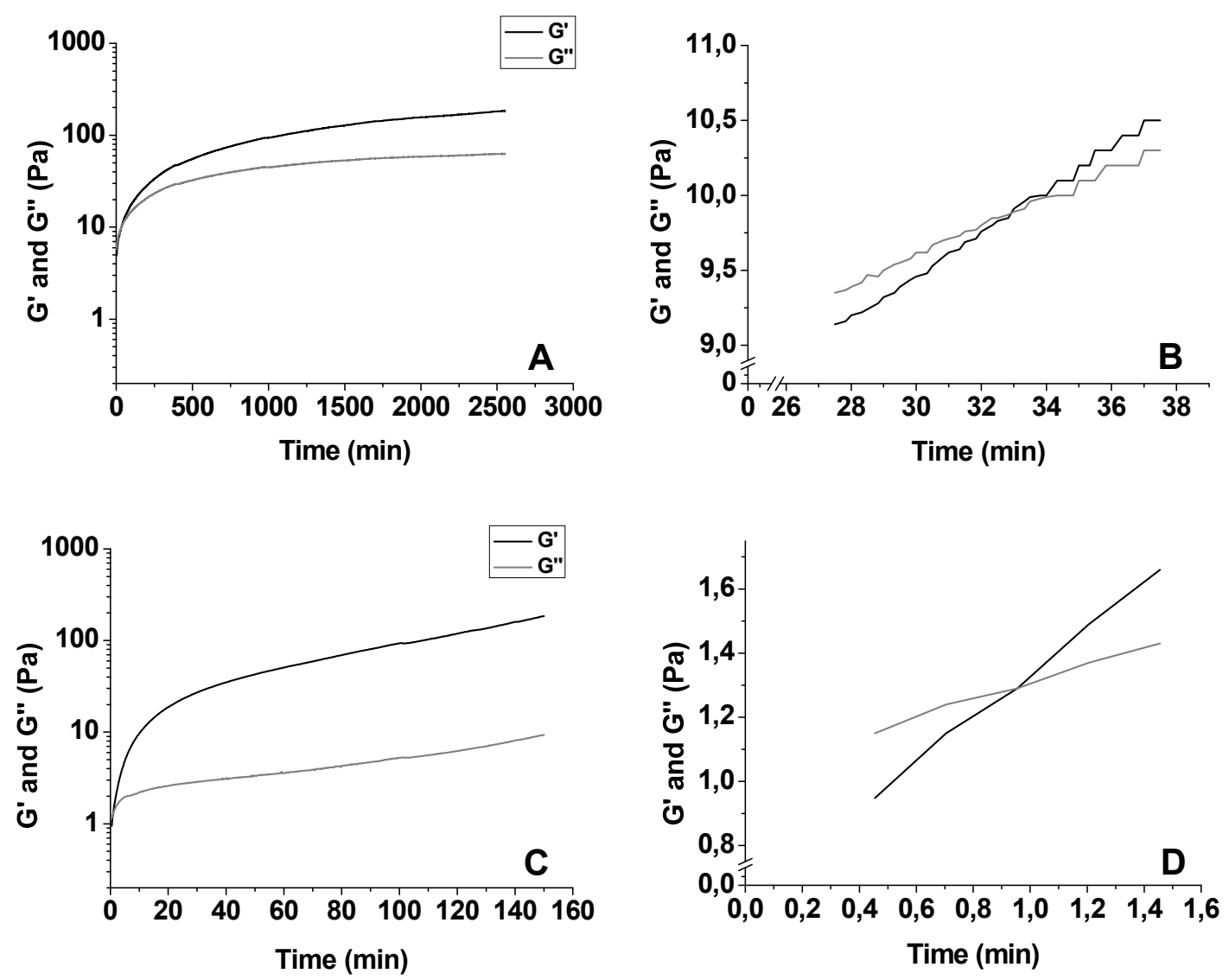

Figure 6. Storage modulus $\left(G^{\prime}\right)$ and loss modulus $\left(G^{\prime \prime}\right)$ as a function of time after mixing $6 \mathrm{w} / \mathrm{v} \%$ macromonomer solutions of opposite chirality at $20{ }^{\circ} \mathrm{C}$. PEG-(OCO)-(PDLA 13$)_{8}+$ PEG-(OCO)$\left(\mathrm{PLLA}_{13}\right)_{8}(\mathrm{~A}, \mathrm{~B})$, PEG-(NHCO)-(PDLA 13$)_{8}+$ PEG-(NHCO)-(PLLA $\left.{ }_{13}\right)_{8}(\mathrm{C}, \mathrm{D})$. Graphs B and D show the crossover points of G' and G', in graphs A and C, respectively.

To investigate the effect of repeated heating cycles on the gel stiffness, PEG-(NHCO)-(PDLA 13$)_{8}$ and PEG-(NHCO)-(PLLA 13$)_{8}$ solutions were mixed at a low concentration of $7 \mathrm{w} / \mathrm{v} \%$, equilibrated for $24 \mathrm{~h}$ and subjected to rheological measurements with temperature profiles as specified in Figure 7A. During the entire measurement G' exceeded G', (G', values not shown), showing that the system retained the gel state. After reaching equilibrium, as shown in interval I, G' is constant. Upon heating the gel from $20{ }^{\circ} \mathrm{C}$ to $60{ }^{\circ} \mathrm{C}$ (interval II) G' decreases rapidly because physical crosslinks are lost due to an increased mobility of the PLA chains. Keeping the temperature constant at $60{ }^{\circ} \mathrm{C}$ for $15 \mathrm{~min}$ (interval III), a large increase in $\mathrm{G}^{\prime}$ is observed. This indicates that new crosslinks are formed which are stable even at $60{ }^{\circ} \mathrm{C}$. During cooling from $60{ }^{\circ} \mathrm{C}$ to $18{ }^{\circ} \mathrm{C}$ (interval 
IV) G' increased likely due to crystallization of the PLA domains. Bringing the system to equilibrium a small decrease of G' can be seen which is mainly due to a shift in temperature up to $22{ }^{\circ} \mathrm{C}$. In this temperature cycle $\mathrm{G}^{\prime}$ thus increases to a value 4 times higher than its initial value. Repeating this temperatures cycle results in consecutive increments in $\mathrm{G}^{\prime}$ and the final value is one order of magnitude higher then the initial value (Figure 7B).
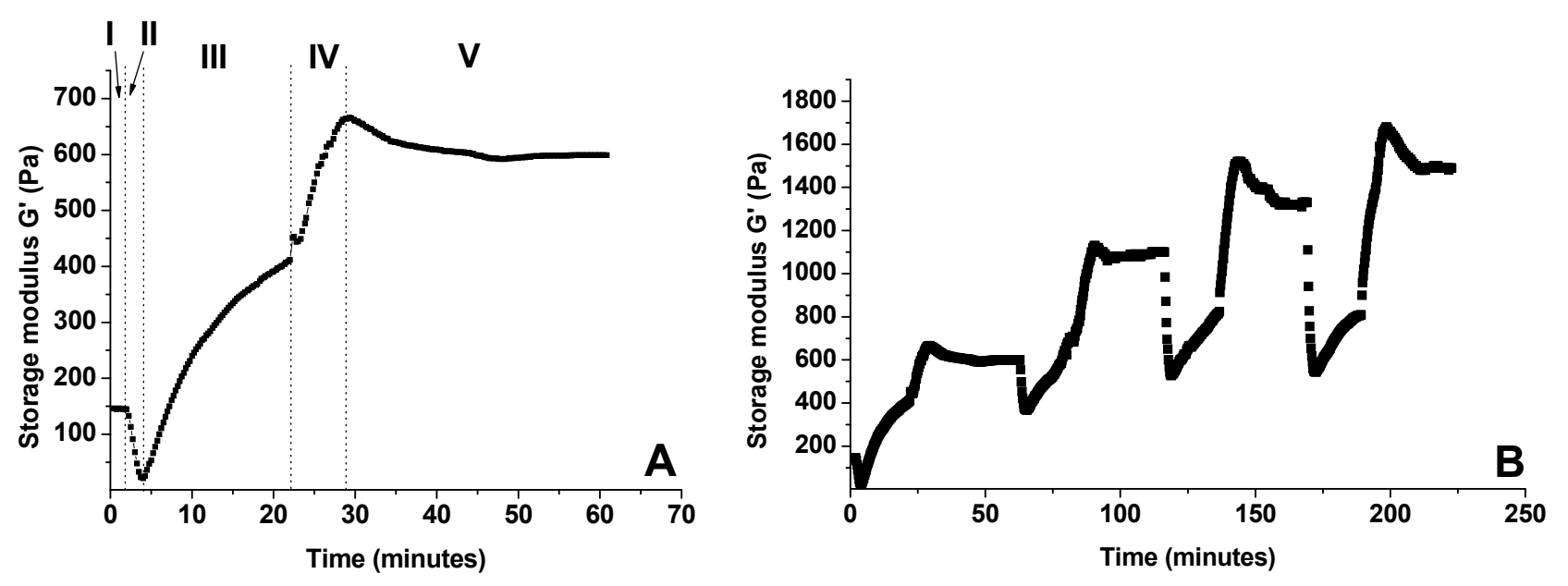

Figure 7. Storage modulus $\left(G^{\prime}\right)$ as a function of time for a $7 \mathrm{w} / \mathrm{v} \%$ stereocomplexed PEG(NHCO)-( $\left.\mathrm{PLA}_{13}\right)_{8}$ hydrogel. After equilibration at $20^{\circ} \mathrm{C}(\mathrm{I})$. Increasing the temperature from $20^{\circ} \mathrm{C}$ to $60{ }^{\circ} \mathrm{C}$ (II). Equilibration at $60{ }^{\circ} \mathrm{C}$ (III). Cooling to $18{ }^{\circ} \mathrm{C}$ (IV). Equilibration at $20{ }^{\circ} \mathrm{C}(\mathrm{V})$. A single temperature cycle (A). Four consecutive temperature cycles (B).

The temperature dependent mechanical properties observed can be illustrated by a mechanism depicted in Figure 8. In aqueous solution the PEG-(PDLA) 8 and PEG-(PLLA) 8 star block copolymers form flower-like micelles and aggregates consisting of a PLA core and a PEG shell. These aggregates do contain PLA blocks dangling in water. ${ }^{18}$ After mixing PEG-(PDLA) 8 and PEG-(PLLA) 8 solutions, stereocomplexation of these dangling PLA chains results in interaggregate bridges. The resulting stereocomplexes lead to spontaneous gel formation at room temperature. Upon heating, the increased mobility of the PLA units facilitates intermicellar and interaggregate bridging to a greater extent resulting in the formation of stereocomplexes which are irreversible. Consequently, the higher strength of the crosslinks results in a stiffer gel as shown by the increased storage modulus. Repeated heating cycles result in higher $G^{\prime}$ values, but the effect becomes progressively smaller (Figure 7B). Because of the high stability of the stereocomplexes formed a temperature irreversible system is established. 


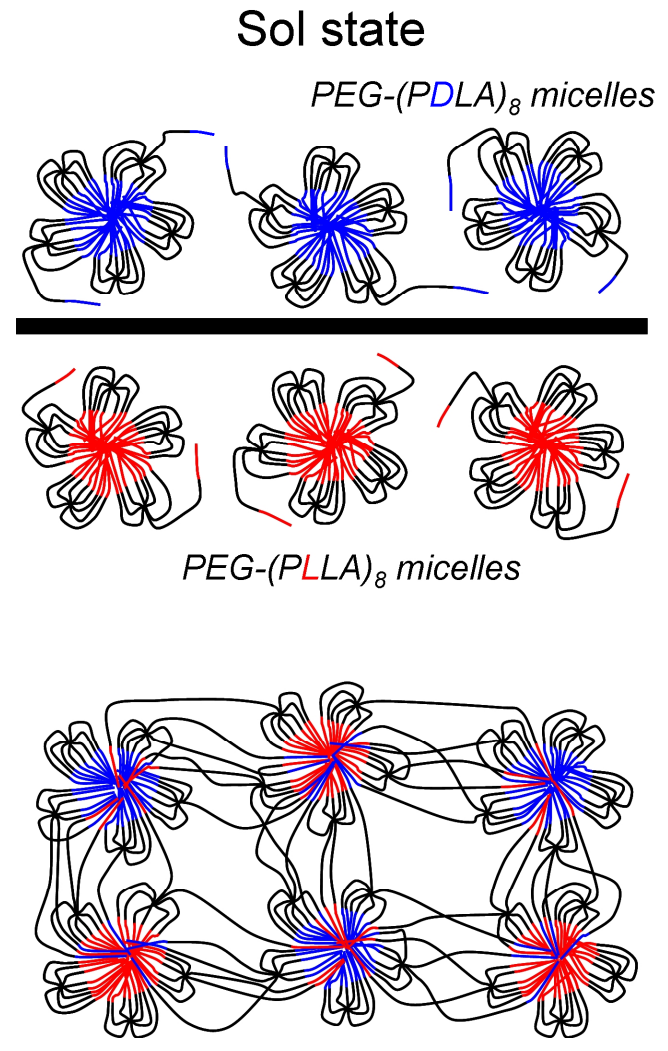

Enhanced gel state
Sol state
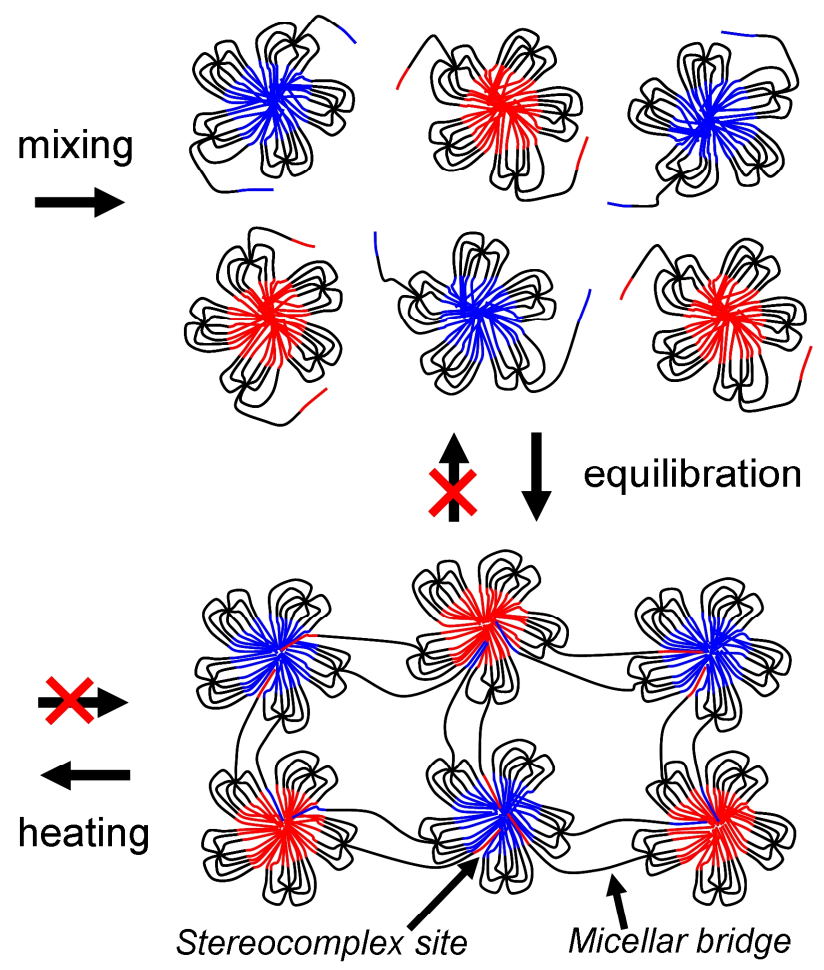

Gel state

Figure 8. Proposed gelation mechanism for an enantiomeric mixture of PEG-(PLA) 8 star block copolymers in water.

Hydrogel degradation/dissolution and release of lysozyme. Stereocomplexed hydrogels were prepared by mixing single enantiomer aqueous solutions and a PBS solution was put on top of the gels. The stereocomplexed PEG-(OCO)-( $\left.\mathrm{PLA}_{13}\right)_{8}$ hydrogel was completely dissolved within $12 \mathrm{~d}$ (Figure 9). The stereocomplexed PEG-(NHCO)-( $\left.\mathrm{PLA}_{13}\right)_{8}$ hydrogel exhibits a significantly higher stability and complete degradation/dissolution is not observed until day 29. Comparing these data to $22 \mathrm{w} / \mathrm{v} \%$ single enantiomer PEG-(OCO)-(PLA $\left.{ }_{13}\right)_{8}$ and PEG-(NHCO)-(PLA 13$)_{8}$ hydrogels, which are stable in vitro for 5 and $15 \mathrm{~d}$ respectively, ${ }^{12}$ shows that stereocomplexation prolongs the stability considerably. Similar findings were reported in a study towards the degradation of films prepared from PDLA/PLLA stereocomplexes or plain PLLA. ${ }^{25}$ The higher hydrolytic stability of the PDLA/PLLA stereocomplexes was attributed to the higher degree of crystallinity as well as to the strong secondary interactions in the stereocomplexed material. In general, physically crosslinked hydrogels eventually dissolve because the micellar and aggregate packing is disrupted as a result of the concentration difference of the copolymer in the hydrogel and the surrounding medium. The higher stability of stereocomplex crystallites in comparison to PLLA amorphous aggregates, which act as crosslinks, most likely leads to less facile disruption of the micellar and aggregate packing. 


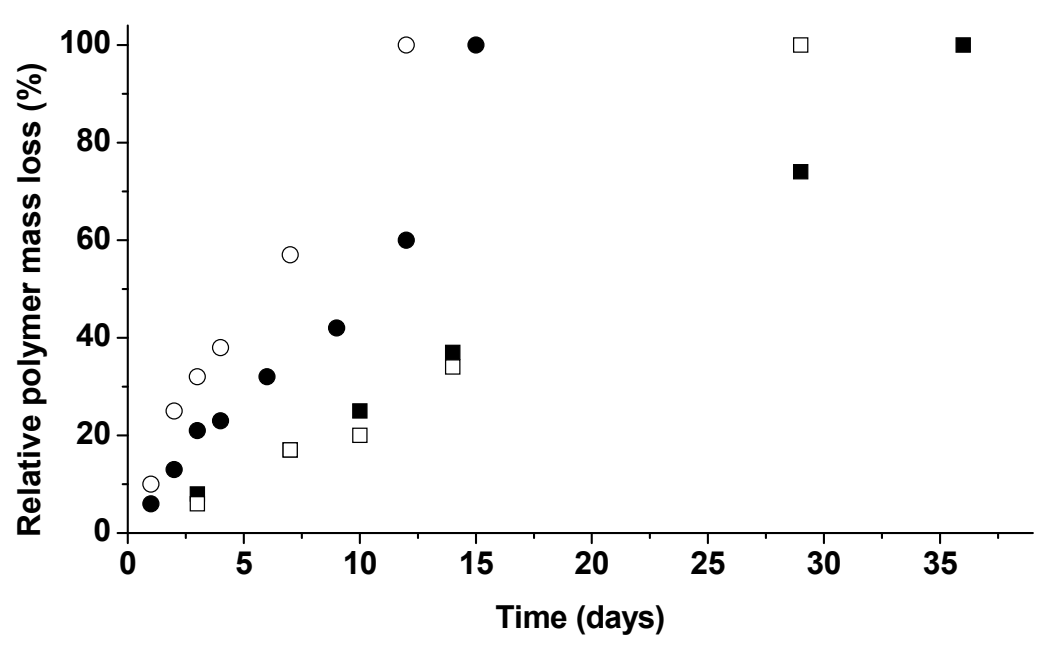

Figure 9. Relative polymer mass loss from $14 \mathrm{w} / \mathrm{v} \%$ stereocomplexed PEG-(NHCO)-(PLA 13$)_{8}$ (squares) and stereocomplexed PEG-(OCO)-(PLA 13$)_{8}$ (circles) hydrogels in PBS at $37{ }^{\circ} \mathrm{C}$. Unloaded hydrogels (open symbols); hydrogels initially loaded with 10 wt \% lysozyme (closed symbols).

Injectable hydrogels are interesting materials to be used in the controlled delivery of biologically active agents. Lysozyme, having a molecular weight of $14 \mathrm{kDa}$, was chosen as a model protein in release experiments with a similar set-up as the degradation experiments. Lysozyme is released from $14 \mathrm{w} / \mathrm{v} \%$ stereocomplexed PEG-(OCO)-(PLA $\left.)_{13}\right)_{8}$ hydrogels up to $15 \mathrm{~d}$ (Figure 10). Gravimetrical degradation/dissolution experiments showed that within this time period also the hydrogel is completely solubilized (Figure 9). Release from the stereocomplexed PEG-(NHCO)$\left(\mathrm{PLA}_{13}\right)_{8}$ hydrogel is considerably more sustained and lysozyme continues to be released until day 36. Also here the hydrogel becomes solubilized within the same time frame. The activity of the released lysozyme was retained, as indicated by bacteria lysis experiments (data not shown).

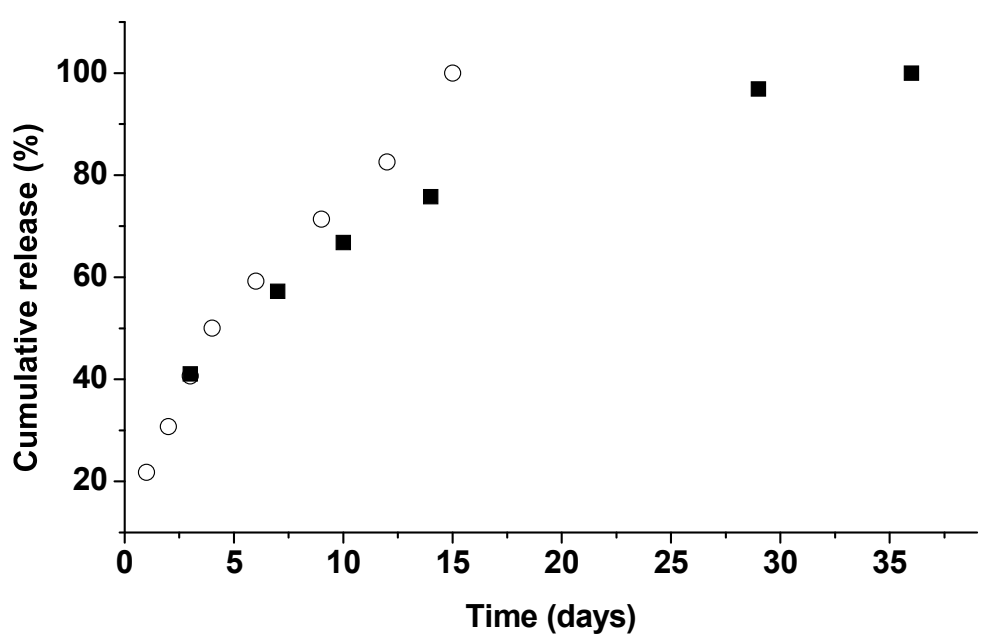

Figure 10. Lysozyme release from stereocomplexed PEG-(NHCO)-(PLA $\left.{ }_{13}\right)_{8}$ hydrogels ( $\left.\mathbf{a}\right)$ and stereocomplexed PEG-(OCO)-(PLA 13$)_{8}$ hydrogels $(\circ)$ in $\mathrm{PBS}$ at $37^{\circ} \mathrm{C}$. 
Interestingly, the presence of lysozyme slightly increases the stability of the hydrogels (Figure 9). Amongst all interactions between a protein and its environment, hydrophobic interactions have been reported to play an important role. ${ }^{26}$ Possibly the hydrophobic domains of lysozyme interact with the PLA arms, resulting in the formation of additional physical crosslink sites. Analysis of degrading hydrogel samples by ${ }^{1} \mathrm{H}$ NMR spectroscopy confirmed that lysozyme has an inhibitory effect on the degradation of the star block copolymers. It was found that the DP in the PLA arms of the unloaded PEG-(NHCO)-(PLA $\left.{ }_{13}\right)_{8}$ hydrogel decreased from 13.3 lactyl units at day 0 to 7.5 lactyl units at day 29 as a result of the hydrolysis of ester groups. In the PEG-(NHCO)-(PLA 13$)_{8}$ hydrogel initially loaded with $10 \mathrm{w} / \mathrm{v} \%$ lysozyme, the degradation was less pronounced as the DP decreased from 13.3 to 9.3 lactyl units after $29 \mathrm{~d}$ in vitro. The release of lysozyme was found to be proportional to the square root of time up to a cumulative release of approximately $85 \%$ for both the stereocomplexed PEG-(NHCO)-(PLA 13$)_{8}$ and the stereocomplexed PEG-(OCO)-(PLA 13$)_{8}$ hydrogel, suggesting that the release is first order from these systems. ${ }^{27}$ However, several other factors influence the release of the lysozyme from the stereocomplexed hydrogels. Hydrolytic degradation in the PLA arms causes removal of physical crosslinks, leading to increased swelling and eventual dissolution of the hydrogel. Although the hydrogel degradation takes place in a similar time period as the lysozyme release, the diffusion coefficient appears not to change considerably and first order kinetics are maintained. Possibly the degradation does not influence the number of effective crosslinks present or an enhanced interaction of the protein with the surrounding matrix becomes important.

${ }^{1} \mathrm{H}$ NMR analysis of remaining polymers in degrading hydrogel samples revealed that degradation in stereocomplexed PEG-(OCO)-(PLA $\left.{ }_{13}\right)_{8}$ hydrogels occurs through preferential hydrolysis of the linking PEG-PLA ester groups. In contrast, the linking amide groups in the stereocomplexed PEG-(NHCO)-( PLA $\left._{13}\right)_{8}$ were found unaffected during the in vitro experiments. Degradation in these systems occurs by hydrolysis of ester groups in the PLA arms. These observations are similar to those on single enantiomer hydrogels ${ }^{12}$ and are most likely the reason for the enhanced stability of stereocomplexed PEG-(NHCO)-(PLA $\left.\mathrm{PL}_{13}\right)_{8}$ hydrogels over stereocomplexed PEG-(OCO)-( $\left.\mathrm{PLA}_{13}\right)_{8}$ hydrogels. The more sustained release of lysozyme from the stereocomplexed PEG-(NHCO)-(PLA 13$)_{8}$ hydrogels (Figure 10) is probably the result of this higher stability.

The stabilizing effect of lysozyme on stereocomplexed hydrogels was also found during oscillatory rheology experiments on degrading gels. Figure 11 shows that during the in vitro experiments the storage modulus G' decreases as a result of the degradation and dissolution of the star block copolymer. It should be noted that for some hydrogel samples, additional rheological measurements at later time points could not be performed because of severe hydrogel degradation. The enhanced mechanical properties of the stereocomplexed PEG-(NHCO)-(PLA $\left.{ }_{13}\right)_{8}$ hydrogels in 
comparison with PEG-(OCO)-( $\left.\mathrm{PLA}_{13}\right)_{8}$ hydrogels (Figure 5) are maintained throughout the degradation period. It follows from Figure 11 that the storage modulus $\mathrm{G}$ ' is significantly higher for hydrogels initially loaded with $10 \mathrm{wt} \%$ lysozyme in comparison with unloaded hydrogels during the entire degradation period. These data provide further evidence that the interaction of lysozyme with the surrounding matrix results in the formation of additional crosslink sites.

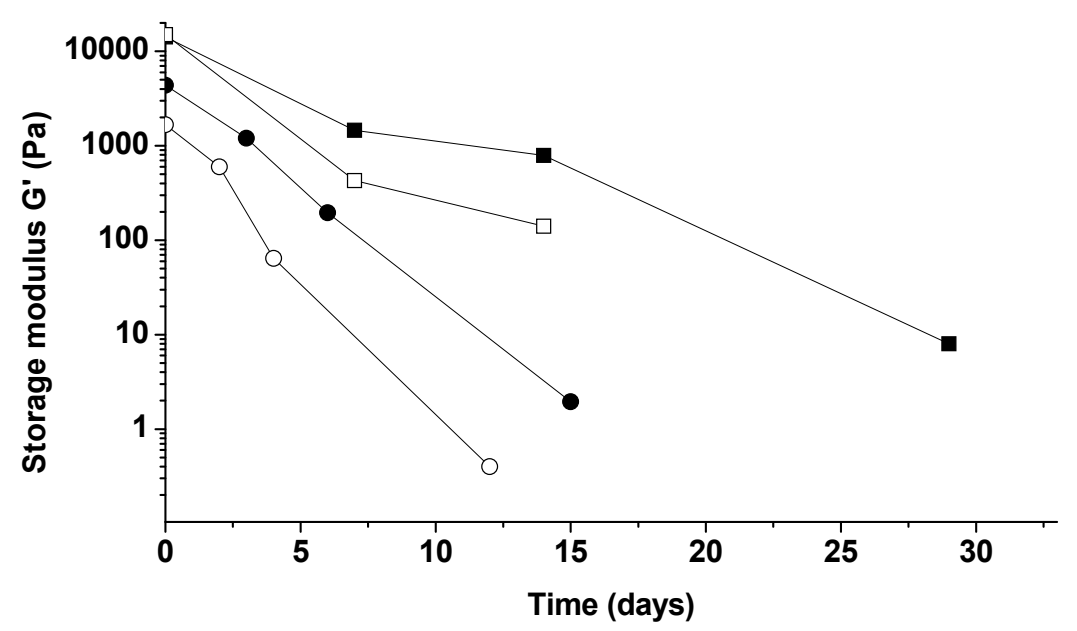

Figure 11. Storage modulus $\left(\mathrm{G}^{\prime}\right)$ as a function of degradation time for stereocomplexed PEG(NHCO)-(PLA $\left.{ }_{13}\right)_{8}$ hydrogels (squares) and stereocomplexed PEG-(OCO)-(PLA 13$)_{8}$ hydrogels (circles) in PBS at $37^{\circ} \mathrm{C}$. Unloaded hydrogels (open symbols); hydrogels initially loaded with 10 wt \% lysozyme (closed symbols).

\subsection{Conclusions}

In the present study, stereocomplexation was shown a facile method for the in situ formation of robust, physically crosslinked hydrogels starting from enantiomeric PEG-(PLA) 8 type copolymer solutions. Upon mixing PEG-(PDLA) 8 and PEG-(PLLA) 8 solutions, interaggregate bridging of dangling PLA chains by the formation of stereocomplexes leads to spontaneous gelation. When these gels are heated, rearrangements leading to an increase in the amount of stereocomplexes take place. ${ }^{1} \mathrm{H}$ NMR experiments revealed that stereocomplexes in the mixed enantiomer PEG-(NHCO)$(\mathrm{PLA})_{8}$ hydrogels are rigid and highly stable up to even $70{ }^{\circ} \mathrm{C}$. Stereocomplexation in these systems thus results in a temperature irreversible gel system. Compared to single enantiomer hydrogels of similar concentration, stereocomplexed PEG-(PLA) 8 hydrogels exhibited enhanced mechanical properties and in vitro stability. By changing the linking unit between the hydrophobic and hydrophilic block from an ester to an amide group in the PEG-(PLA) 8 copolymers gels can be formed at lower polymer concentration, showing a larger gel window and improved mechanical properties. Moreover, because stereocomplexed PEG-(NHCO)-(PLA) 8 hydrogels are more stable towards hydrolysis than stereocomplexed PEG-(OCO)-(PLA) $)_{8}$ hydrogels a prolonged release of the small protein lysozyme was found. The robust mechanical properties, the possibility to be formed $i n$ 
situ and the stability towards hydrolytic degradation make the stereocomplexed PEG-(NHCO)$(\mathrm{PLA})_{8}$ hydrogels interesting for biomedical applications such as controlled drug delivery systems and matrices for tissue engineering.

\subsection{References}

[1] Zisch, A. H.; Lutolf, M. P.; Hubbell, J. A. Cardiovasc. Pathol. 2003, 12, 295-310.

[2] Peppas, N. A.; Bures, P.; Leobandung, W.; Ichikawa, H. Eur. J. Pharm. Biopharm. 2000, 50, 27-46.

[3] Peppas, N. A.; Khare, A. R. Adv. Drug Delivery Rev. 1993, 11, 1-35.

[4] Ruel-Gariepy, E.; Leroux, J. C. Eur. J. Pharm. Biopharm. 2004, 58, 409-426.

[5] Fujiwara, T.; Mukose, T.; Yamaoka, T.; Yamane, H.; Sakurai, S.; Kimura, Y. Macromol. Biosci. 2001, 1, 204-208.

[6] Mukose, T.; Fujiwara, T.; Nakano, J.; Taniguchi, I.; Miyamoto, M.; Kimura, Y.; Teraoka, I.; Lee, C. W. Macromol. Biosci. 2004, 4, 361-367.

[7] Nagahama, K.; Fujiura, K.; Enami, S.; Ouchi, T.; Ohya, Y. J. Polym. Sci., Part A: Polym. Chem. 2008, 46, 6317-6332.

[8] Li, S.; El Ghzaoui, A.; Dewinck, E. Macromol. Symp. 2005, 222, 23-35.

[9] Zhang, Y.; Wu, X.; Han, Y.; Mo, F.; Duan, Y.; Li, S. Int. J. Pharm. 2010, 386, 15-22.

[10] Hiemstra, C.; Zhong, Z.; Li, L. B.; Dijkstra, P. J.; Feijen, J. Biomacromolecules 2006, 7, 2790-2795.

[11] Hiemstra, C.; Zhong, Z.; Van Tomme, S. R.; van Steenbergen, M. J.; Jacobs, J. J. L.; Den Otter, W.; Hennink, W. E.; Feijen, J. J. Controlled Release 2007, 119, 320-327.

[12] Buwalda, S. J.; Dijkstra, P. J.; Calucci, L.; Forte, C.; Feijen, J. Biomacromolecules 2010, 11, 224-232.

[13] Kim, B. S.; Oh, J. M.; Cho, J. S.; Lee, S. H.; Lee, B.; Khang, G.; Lee, H. B.; Kim, M. S. J. Appl. Polym. Sci. 2009, 111, 1706-1712.

[14] Gerhards, R.; Dietrich, W. J. Magn. Reson. 1976, 23, 21-29.

[15] Shih, P.; Malcolm, B. A.; Rosenberg, S.; Kirsch, J. F.; Wilson, A. C. Methods Enzymol. 1993, 224, 576-590.

[16] De Jong, S. J.; de Smedt, S. C.; Wahls, M. W. C.; Demeester, J.; Kettenes-van den Bosch, J. J.; Hennink, W. E. Macromolecules 2000, 33, 3680-3686.

[17] Portinha, D.; Bouteiller, L.; Pensec, S.; Richez, A.; Chassenieux, C. Macromolecules 2004, 37, 3401-3406.

[18] Calucci, L.; Forte, C.; Buwalda, S. J.; Dijkstra, P. J.; Feijen, J. Langmuir 2010, 26, 12890-12896.

[19] Tsuji, H. Macromol. Biosci. 2005, 5, 569-597.

[20] Liu, K. J.; Ullman, R. J. Chem. Phys. 1968, 48, 1158-1168.

[21] Hey, M. J.; Ilett, S. M.; Davidson, G. J. Chem. Soc., Faraday Trans. 1995, 91, 38973900.

[22] Tung, C. Y. M.; Dynes, P. J. J. Appl. Polym. Sci. 1982, 27, 569-574.

[23] Piepenbrock, M. O. M.; Lloyd, G. O.; Clarke, N.; Steed, J. W. Chem. Rev. 2010, 110, 1960-2004.

[24] Velthoen, I. W.; Tijsma, E. J.; Dijkstra, P. J.; Feijen, J. Macromol. Symp. 2008, 272, 1327.

[25] Andersson, S. R.; Hakkarainen, M.; Inkinen, S.; Sodergard, A.; Albertsson, A. C. Biomacromolecules 2010, 11, 1067-1073.

[26] Nakanishi, K.; Sakiyama, T.; Imamura, K. J. Biosci. Bioeng. 2001, 91, 233-244.

[27] Siepmann, J.; Peppas, N. A. Adv. Drug Delivery Rev. 2001, 48, 139-157. 
Chapter 5

\title{
Self-assembly and photocrosslinking of 8-armed PEG-PTMC star block copolymers
}

This chapter has been published: Sytze J. Buwalda, ${ }^{a}$ Laura B. Perez, ${ }^{\text {a }}$ Sandra Teixeira, ${ }^{\text {a }}$ Lucia Calucci, ${ }^{b}$ Claudia Forte, ${ }^{b}$ Jan Feijen, ${ }^{a}$ and Pieter J. Dijkstra ${ }^{a}$ Biomacromolecules 2011, 12, 27462754.

${ }^{a}$ Department of Polymer Chemistry and Biomaterials, Faculty of Science and Technology, MIRA Institute for Biomedical Technology and Technical Medicine, University of Twente, P.O. Box 217, 7500 AE Enschede, The Netherlands

${ }^{\mathrm{b}}$ Istituto di Chimica dei Composti OrganoMetallici, CNR-Consiglio Nazionale delle Ricerche, Area della Ricerca di Pisa, via G. Moruzzi 1, 56124 Pisa, Italy

\begin{abstract}
Eight-armed poly(ethylene glycol)-poly(trimethylene carbonate) star block copolymers (PEG$(\mathrm{PTMC})_{8}$ ) linked by a carbamate group between the PEG core and the PTMC blocks were synthesized by the metal-free, $\mathrm{HCl}$ catalyzed ring opening polymerization of trimethylene carbonate using an amine terminated 8-armed star PEG in dichloromethane. Although dye solubilization experiments, nuclear magnetic resonance spectroscopy and dynamic light scattering clearly indicated the presence of aggregates in aqueous dispersions of the copolymers, no physical gelation was observed up to high concentrations. PEG-(PTMC $)_{8}$ was end group functionalized using acryloyl chloride and photopolymerized in the presence of Irgacure 2959. When dilute aqueous dispersions of PEG-(PTMC $)_{8}$-AC were UV irradiated, chemically crosslinked PEG-PTMC nanoparticles were obtained, whereas irradiation of more concentrated PEG-(PTMC 9$)_{8}$-AC dispersions resulted in the formation of photocrosslinked hydrogels. Their good mechanical properties and high stability against hydrolytic degradation make photocrosslinked PEG-PTMC hydrogels interesting for biomedical applications such as matrices for tissue engineering and controlled drug delivery systems.
\end{abstract}




\subsection{Introduction}

Hydrogels are polymer networks that receive much interest for their use in biomedical applications because they generally exhibit excellent biocompatibility due to their high water content. ${ }^{1-3}$ Chemically crosslinked hydrogels are generally more stable and have better mechanical properties compared to physically crosslinked systems. Poly(ethylene glycol) (PEG) is a widely used hydrophilic component in hydrogels because of its good biocompatibility. As a hydrophobic component, aliphatic polyesters such as poly(lactide) (PLA) and poly( $\varepsilon$-caprolactone) (PCL) have been investigated extensively in block copolymers with PEG for the preparation of physically crosslinked hydrogels. Chemically crosslinked hydrogels are often prepared by photocuring of polymers which are functionalized with e.g. acrylic end groups. ${ }^{4}$ Hubbell et al. prepared photocrosslinked hydrogels from end acrylated PLA-PEG-PLA and PLGA-PEG-PLGA triblock copolymers. ${ }^{5}$ The degradation times of the hydrogels varied from 0.3 to $120 \mathrm{~d}$ depending on the molecular weight of the PEG and the length and nature of the hydrophobic polyester block. A drawback of hydrogels based on PEG-polyester copolymers is the formation of acidic components upon degradation, which may deactivate $\mathrm{pH}$-sensitive compounds such as growth and differentiation factors. ${ }^{6}$ In this respect poly(trimethylene carbonate) (PTMC) is an interesting alternative for it is enzymatically degraded in vivo with the formation of water soluble and nonacidic products such as 1,3-propanediol, carbon dioxide and PTMC oligomers. ${ }^{7}$ During the last decade, much effort has been devoted to the synthesis of PEG-PTMC block copolymers. Most often stannous octoate is used as the catalyst for the ring opening polymerization of TMC initiated by hydroxyl end groups of PEG. Recently, low molecular weight PEG-PTMC block copolymers were prepared by the ring opening polymerization of TMC from hydroxyl functional PEGs and $\mathrm{HCl}$ as a catalyst via an activated monomer mechanism. ${ }^{8}$ This method avoids the use of potentially cytotoxic organometallic catalysts and is preferred when the resultant polymers are to be used in biomedical applications. ${ }^{9}$ Using mono-, di- or multifunctional PEGs, polymers with one, two, four or eight PTMC arms were prepared with constant hydrophobic content (50 wt \% PTMC) and a total molecular weight of $4000 \mathrm{~g} / \mathrm{mol}^{8}{ }^{8}$ The critical association concentration (CAC) of these polymers as well as the aggregate diameter in aqueous solution increased with increasing arm number, which was attributed to hampered hydrophobic interactions. Temperature dependent gelation of PEGPTMC diblock and PTMC-PEG-PTMC triblock copolymers has been shown by Kim et al. ${ }^{10}$ and Bat et al., ${ }^{11}$ respectively. For the diblock polymers the transition temperature could be controlled in a range of $20-75^{\circ} \mathrm{C}$ by varying the polymer concentration, molecular weight and composition of the polymer. In the triblock system, sol to gel transitions were observed for polymers with a PEG content between 68 and $82 \mathrm{wt} \%$. Gelation only occurred for polymers with a $\mathrm{M}_{\mathrm{n}}$ of $14 \mathrm{~kg} / \mathrm{mol}$ and higher, indicating that the total molecular weight is an important polymer property for the temperature dependent gelation behavior. Whereas diblock copolymers afford hydrogels with low 
storage moduli, PTMC-PEG-PTMC triblock copolymer hydrogels at similar polymer concentrations showed storage moduli up to $3 \mathrm{kPa}$.

Chemically crosslinked hydrogels based on PEG and PTMC only have been prepared from low molecular weight triblock copolymers by end group acrylation and subsequent photopolymerization. ${ }^{12}$ Upon immersion in PBS at $37^{\circ} \mathrm{C}$, a photocrosslinked PEG-PTMC triblock copolymer (600-1000-600 g/mol) exhibited a mass loss of $7 \%$ after 4 weeks as a result of bulk degradation.

In previous research we have shown that 8-armed PEG-PLA star block copolymers yield physically crosslinked hydrogels at much lower concentrations compared to linear PLA-PEG-PLA triblock copolymers. ${ }^{13}$ This prompted us to investigate the aggregation behavior of analogous PEGPTMC star block copolymers in aqueous solutions. In this paper, we describe the synthesis of 8armed PEG-PTMC star block copolymer by metal-free ring opening polymerization of TMC initiated by amine end functionalized 8-armed PEG. The self-assembly of the star polymers in water was studied. It was found that stable bridging between polymer aggregates, necessary to form a hydrogel, is disfavored due to the high mobility of the PTMC blocks. We show that interaggregate bridging can however be stabilized by UV crosslinking; an acrylated 8-armed PEG-PTMC star block copolymer was used to prepare a photocrosslinked hydrogel or nanoparticles by UV irradiation. The physical, mechanical and degradation properties of the hydrogel were investigated, as well as its biocompatibility.

\subsection{Experimental section}

Materials. Hydroxyl terminated 8-armed poly(ethylene glycol) (PEG-(OH) $8, M_{n}$, NMR $=21400$ $\mathrm{g} / \mathrm{mol}$ ) was purchased from Jenkem (Allen, Texas, USA) and purified before use by dissolution in dichloromethane and precipitation in cold diethyl ether. The PEG- $(\mathrm{OH})_{8}$ was converted in PEG$\left(\mathrm{NH}_{2}\right)_{8}$ following a two-step procedure analogous to that described by Elbert and Hubbell for linear hydroxyl terminated PEGs. ${ }^{14}$ Trimethylene carbonate (TMC) was obtained from Boehringer (Ingelheim, Germany) and used as received. $\mathrm{HCl}(1.0 \mathrm{M}$ solution in diethyl ether), acryloyl chloride, methanesulfonyl chloride (mesyl chloride), triethylamine (TEA), lipase from porcine pancreas and $25 \%$ aqueous ammonia solution were from Sigma-Aldrich (St Louis, Missouri, USA). Toluene, hexane, diethyl ether, methanol and dichloromethane were all from Biosolve (Valkenswaard, The Netherlands). Dichloromethane, TEA and toluene were dried over calcium hydride, potassium hydroxide and sodium, respectively, and distilled prior to use. Irgacure 2959 was obtained from Ciba (Basel, Switzerland). Chondrocytes from bovine cartilage were used for cytotoxicity measurements in this study. The cells were cultured at $37^{\circ} \mathrm{C}$ in a humidified atmosphere of $5 \% \mathrm{CO}_{2}$ in air in $175 \mathrm{~cm}^{2}$ flasks containing Dulbecco's Modified Eagle Medium (DMEM, Gibco) supplemented with $10 \%$ fetal bovine serum (Gibco) and $1 \%$ 
penicillin/streptomycin (Gibco). The medium was refreshed twice a week until cells reached confluency. When confluent, cells were detached using trypsin solution $(0.25 \%$ trypsin $/ 0.05 \%$ EDTA, Invitrogen), and diluted 1:5 in a fresh culture flask. In this study, cells from passage 2 were used.

Synthesis. The 8-armed poly(ethylene glycol)-poly(trimethylene carbonate) star block copolymer $\left(\mathrm{PEG}-\left(\mathrm{PTMC}_{9}\right)_{8}\right)$ was synthesized by the ring opening polymerization of trimethylene carbonate using amine terminated 8-armed star PEG $\left(\mathrm{PEG}-\left(\mathrm{NH}_{2}\right)_{8}\right)$ as an initiator and $\mathrm{HCl}$ as a catalyst in dichloromethane at room temperature.

PEG-(NH$)_{8}(5 \mathrm{~g}, 0.23 \mathrm{mmol})$ and toluene $(15 \mathrm{ml})$ were introduced in a round bottomed flask. The toluene was distilled off and $\mathrm{CH}_{2} \mathrm{Cl}_{2}(5 \mathrm{ml})$ and TMC $(2.04 \mathrm{~g}, 20 \mathrm{mmol})$ were added. The polymerization was started by the addition of $1 \mathrm{M} \mathrm{HCl}$ solution in diethyl ether $(8 \mathrm{ml}, 8 \mathrm{mmol})$ at $25{ }^{\circ} \mathrm{C}$ in a nitrogen atmosphere. After $48 \mathrm{~h}$, the product was purified by precipitation in a 20 -fold excess of a mixture of diethyl ether and methanol $(40 / 1 \mathrm{v} / \mathrm{v})$. PEG-(PTMC 9$)_{8}$ was obtained by filtration and dried under vacuum at ambient temperature overnight. ${ }^{1} \mathrm{H}$ NMR $\left(\mathrm{CDCl}_{3}\right): \delta=7.92(\mathrm{~s}$, $\mathrm{CH}_{2} \mathrm{NHCOO}$ ), 4.28 (t, $\left.\mathrm{CH}_{2} \mathrm{NHCOO}\right), 4.22$ (t, $\left.\mathrm{OCH}_{2} \mathrm{CH}_{2} \mathrm{CH}_{2} \mathrm{O}\right), 3.72$ (t, $\left.\mathrm{CH}_{2} \mathrm{CH}_{2} \mathrm{OH}\right), 3.63(\mathrm{PEG}$ protons), 2.03 (q, $\mathrm{OCH}_{2} \mathrm{CH}_{2} \mathrm{CH}_{2} \mathrm{O}$ ), 1.90 (q, $\mathrm{CH}_{2} \mathrm{CH}_{2} \mathrm{OH}$ ).

PEG-(PTMC $)_{8}$ star block copolymer was functionalized with acrylate end groups (PEG$\left.\left(\mathrm{PTMC}_{9}\right)_{8} \mathrm{AC}\right)$ according to a procedure described in literature. ${ }^{12}$ PEG-(PTMC 9$)_{8}(6.70 \mathrm{~g}, 0.24$ mmol) was dissolved in $15 \mathrm{ml}$ of dichloromethane. Acryloyl chloride (3.23 ml, $40 \mathrm{mmol}$ ) was added and the reaction mixture was stirred under a $\mathrm{N}_{2}$ atmosphere for $16 \mathrm{~h}$ at $50{ }^{\circ} \mathrm{C}$. The product was precipitated in a 20-times excess of cold hexane and dried under vacuum for $1 \mathrm{~d}$. ${ }^{1} \mathrm{H}$ NMR $\left(\mathrm{CDCl}_{3}\right): \delta=6.83\left(\mathrm{~s}, \mathrm{CH}_{2} \mathrm{NHCOO}\right), 6.41\left(\mathrm{dd}, \mathrm{OCOCHCH}_{2}\right), 6.10$ (dd, $\left.\mathrm{OCOCHCH}_{2}\right), 5.84$ (dd, $\mathrm{OCOCHCH}_{2}$ ), 4.23 ( $\mathrm{t}, \mathrm{OCH}_{2} \mathrm{CH}_{2} \mathrm{CH}_{2} \mathrm{O}$ ), 3.80 (t, $\mathrm{CH}_{2} \mathrm{CH}_{2} \mathrm{OH}$ ), 3.63 (PEG protons), 2.79 and 2.65 (t, $\mathrm{CH}_{2} \mathrm{CH}_{2} \mathrm{OH}$ ), 2.04 (q, $\mathrm{OCH}_{2} \mathrm{CH}_{2} \mathrm{CH}_{2} \mathrm{O}$ ).

Chemically crosslinked PEG-PTMC nanoparticles were prepared by UV irradiation of PEG$\left(\mathrm{PTMC}_{9}\right)_{8}$ - AC star block copolymer. In a typical procedure, a $1 \mathrm{w} / \mathrm{v} \%$ mixture of PEG-(PTMC 9$)_{8}$ AC in water containing $10 \mathrm{~mol} \%$ Irgacure 2959 photoinitiator relative to acrylate groups was prepared. The mixture was irradiated by UV light $\left(\sim 5 \mathrm{~mW} / \mathrm{cm}^{2}\right)$ at $365 \mathrm{~nm}$ for $2 \mathrm{~h}$. Chemically crosslinked PEG-PTMC hydrogels were prepared similarly. Typically, a $40 \mathrm{w} / \mathrm{v} \%$ mixture of PEG$\left(\mathrm{PTMC}_{9}\right)_{8} \mathrm{AC}$ in water containing $10 \mathrm{~mol} \%$ photoinitiator relative to acrylate groups was prepared. The mixture was irradiated by UV light at $365 \mathrm{~nm}$ for $2 \mathrm{~h}$.

Characterization. ${ }^{1} \mathrm{H}$ NMR $(300 \mathrm{MHz})$ spectra were recorded on a Varian Inova $300 \mathrm{NMR}$ spectrometer. Polymers were dissolved in $\mathrm{CDCl}_{3}$ at a concentration of $15 \mathrm{mg} / \mathrm{ml}$. For NMR analysis 
of PEG-PTMC nanoparticles, an aqueous PEG-PTMC nanoparticle dispersion (1 w/v \%) was freeze-dried and the particles were redispersed in $\mathrm{CDCl}_{3}$ at a concentration of $15 \mathrm{mg} / \mathrm{ml}$.

Aqueous solution properties. The critical association concentration (CAC) value of the PEG$\left(\mathrm{PTMC}_{9}\right)_{8}$ star block copolymer in water was determined using the 1,6-diphenyl-1,3,5-hexatriene (DPH) dye solubilization method. ${ }^{15}$ Mixtures of PEG-(PTMC 9$)_{8}$ in distilled water were prepared in the concentration range of $1 \cdot 10^{-6}$ to $5 \mathrm{w} / \mathrm{v} \%$. A solution of DPH was prepared in methanol at a concentration of $0.5 \mathrm{mM}$. Approximately $1 \mathrm{ml}$ of the polymer dispersion was added to a polystyrene vial, followed by addition of $10 \mu \mathrm{l}$ of the DPH solution. The samples were allowed to equilibrate for at least $3 \mathrm{~h}$ in the dark, after which the absorption at $357 \mathrm{~nm}$ relative to a blank (polymer dispersion containing no DPH) was measured using a Varian Cary 300 Bio UV-visible spectrophotometer. The absorption was plotted against the logarithm of the polymer concentration and the intercept of the extrapolated straight lines was taken as the CAC.

Dynamic light scattering (DLS) of the PEG-(PTMC $)_{8}$ star block copolymer in water $\left(1 \cdot 10^{-4}-10\right.$ w/v \%) was performed to determine aggregate sizes. Experiments were carried out between $25{ }^{\circ} \mathrm{C}$ and $55^{\circ} \mathrm{C}$ using a Malvern Nano ZS, a laser wavelength of $633 \mathrm{~nm}$ and a scattering angle of $173^{\circ}$. At each temperature, the sample was allowed to equilibrate for $5 \mathrm{~min}$.

NMR experiments were carried out on a Bruker AMX-300 WB spectrometer working at 300.13 $\mathrm{MHz}$ for proton and at $75.47 \mathrm{MHz}$ for carbon-13 using either a $5 \mathrm{~mm}$ probe head or a $4 \mathrm{~mm}$ $\mathrm{CP} / \mathrm{MAS}$ probe head for solution state and solid state measurements, respectively. The temperature was controlled within $0.1^{\circ} \mathrm{C}$.

Solution state ${ }^{1} \mathrm{H}$ NMR experiments were performed on samples of $0.1,1$, and $10 \mathrm{w} / \mathrm{v} \%$ PEG$\left(\mathrm{PTMC}_{9}\right)_{8}$ in $\mathrm{D}_{2} \mathrm{O}\left(99.98 \% \mathrm{D}\right.$, Eurisotop) with a $90^{\circ}$ pulse of $5.7 \mu \mathrm{s}$ and a recycle delay of $10 \mathrm{~s} ; 8$ to 4000 scans were acquired, depending on sample concentration. The samples were prepared by dissolving the appropriate amount of copolymer in $\mathrm{D}_{2} \mathrm{O}$. Relative peak intensities within each spectrum were determined by integration of the peaks obtained by spectral deconvolution using the SPORT-NMR software, ${ }^{16}$ and were calibrated using 3-(trimethylsilyl)-1-propanesulfonic acid sodium salt (DSS) at known concentration as internal standard. DSS was also used as reference for the ${ }^{1} \mathrm{H}$ and ${ }^{13} \mathrm{C}$ chemical shifts. Solution state ${ }^{13} \mathrm{C}$ NMR experiments were performed on the $10 \mathrm{w} / \mathrm{v}$ $\%$ sample of PEG-(PTMC 9$)_{8}$ in $\mathrm{D}_{2} \mathrm{O}$ with a pulse of $4 \mu \mathrm{s}$, corresponding to a $40^{\circ}$ pulse, and a recycle delay of $30 \mathrm{~s}$, and acquiring 2400 scans.

Solid state ${ }^{13} \mathrm{C}$ magic angle spinning (MAS) NMR experiments were performed under proton decoupling conditions on a dry PEG-(PTMC 9$)_{8}$ sample and on a $50 \mathrm{w} / \mathrm{v} \%$ sample of PEG$\left(\mathrm{PTMC}_{9}\right)_{8}$ in $\mathrm{D}_{2} \mathrm{O}$. In the ${ }^{13} \mathrm{C}$ direct excitation (DE) experiments the $90^{\circ}$ pulse length was $4 \mu \mathrm{s}$ and recycle delays of 12 and $120 \mathrm{~s}$ were used. In the ${ }^{1} \mathrm{H}-{ }^{13} \mathrm{C}$ cross polarization $(\mathrm{CP})$ experiments the ${ }^{1} \mathrm{H}$ 
$90^{\circ}$ pulse length was $4 \mu \mathrm{s}$ and a contact time of $1 \mathrm{~ms}$ and a recycle delay of $12 \mathrm{~s}$ were used. The MAS spinning rate was $3 \mathrm{kHz}$ and $1 \mathrm{kHz}$ for the dry and hydrated samples, respectively.

Hydrogel properties. Oscillatory rheology experiments were performed to determine the mechanical properties of the PEG-PTMC hydrogels. The storage $\left(G^{\prime}\right)$ and loss $\left(G^{\prime \prime}\right)$ modulus of hydrogels were monitored for $12.5 \mathrm{~min}$ at $25^{\circ} \mathrm{C}$ on an Anton-Paar Physica MCR 301 rheometer. Experiments were performed using a flat plate measuring geometry (diameter $25 \mathrm{~mm}$, gap $0.3 \mathrm{~mm}$ ) utilizing a strain of $1 \%$ and a frequency of $1 \mathrm{~Hz}$. To prevent water evaporation, a solvent trap was placed over the geometry.

Hydrolytic and enzymatic degradation experiments were performed to determine the stability of the hydrogels. Freshly prepared hydrogel samples were dried in air and the initial weight $\mathrm{W}_{0}$ was determined. The samples were immersed in PBS at $37{ }^{\circ} \mathrm{C}$. To prevent bacterial growth, $0.02 \mathrm{w} / \mathrm{v} \%$ $\mathrm{NaN}_{3}$ was added to the buffer solution. At regular times, samples were taken out, wiped with tissue paper, and their mass in the swollen state $\left(\mathrm{W}_{\mathrm{s}}\right)$ was determined. Subsequently, the samples were allowed to dry in air overnight to yield the dry weight $\left(\mathrm{W}_{\mathrm{D}}\right)$. The degree of swelling during degradation was calculated from:

$$
\text { degree of swelling }=\frac{\left(\mathrm{W}_{\mathrm{S}}-\mathrm{W}_{\mathrm{D}}\right)}{\mathrm{W}_{\mathrm{D}}} \cdot 100 \%
$$

The remaining relative polymer mass during degradation was calculated from:

$$
\text { relative polymer mass }=\frac{\mathrm{W}_{\mathrm{D}}}{\mathrm{W}_{0}} \cdot 100 \%
$$

Enzymatic degradation was studied by immersing hydrogel samples in PBS containing $1 \mathrm{w} / \mathrm{v} \%$ lipase. The remaining relative polymer mass was determined as described above. All degradation experiments were performed in duplo.

The direct cell contact method was employed to assess the cytotoxicity of photocrosslinked PEGPTMC hydrogels. ${ }^{17}$ Chondrocytes from bovine cartilage $\left(6 \cdot 10^{5}\right.$ cells $\left./ \mathrm{ml}\right)$ were seeded in $50 \mu \mathrm{l}$ DMEM in a 96-well culture plate. Plates were incubated at $37{ }^{\circ} \mathrm{C}$ in a humidified $5 \% \mathrm{CO}_{2}$ atmosphere until the cells formed a monolayer. The culture medium was aspirated and hydrogel pieces $(1 \times 1 \times 1 \mathrm{~mm})$, pre-swollen in culture medium, were placed carefully on the monolayer in $200 \mu \mathrm{l}$ culture medium. The cell viability after $20 \mathrm{~h}$ was determined by the colorimetric MTS assay, according to the manufacturer's instructions (CellTiter 96 Aqueous One Solution Cell Proliferation Assay, Promega). Briefly, MTS solution was added to the wells (20 $\mu 1$ to $100 \mu 1$ of medium) and incubated for $2 \mathrm{~h}$ at $37^{\circ} \mathrm{C}$ in the dark. Afterwards, the supernatant of each sample was collected and its absorbance was measured at $540 \mathrm{~nm}$ using a Tecan Safire microplate reader. Data $(\mathrm{n}=3)$ are expressed as mean \pm standard deviation (SD). 
The morphology of chemically crosslinked PEG-PTMC nanoparticles was evaluated by scanning electron microscopy (SEM). One drop of a nanoparticle dispersion in water was applied to a carbon-coated grid and the water was evaporated overnight in air. Images were obtained with a scanning electron microscope (LEO Gemini 1550 FEG-SEM) at $1.6 \mathrm{kV}$.

\subsection{Results and discussion}

Synthesis. The acid catalyzed ring opening polymerization of TMC in the presence of alcohols as initiators is a highly efficient method recently evaluated. ${ }^{18}$ Moreover, this method avoids the use of metal based catalysts which may cause adverse effects when these materials are used in biomedical applications. The $\mathrm{HCl}$ catalyzed ring opening polymerization of TMC also appeared a convenient method to prepare PEG-PTMC block copolymers when amine end group functionalized 8-armed star PEG was used as an initiator (Figure 1). In this case, the blocks are connected through carbamate bonds. The ring opening polymerization was performed in dichloromethane at room temperature in high yield $(>95 \%)$.

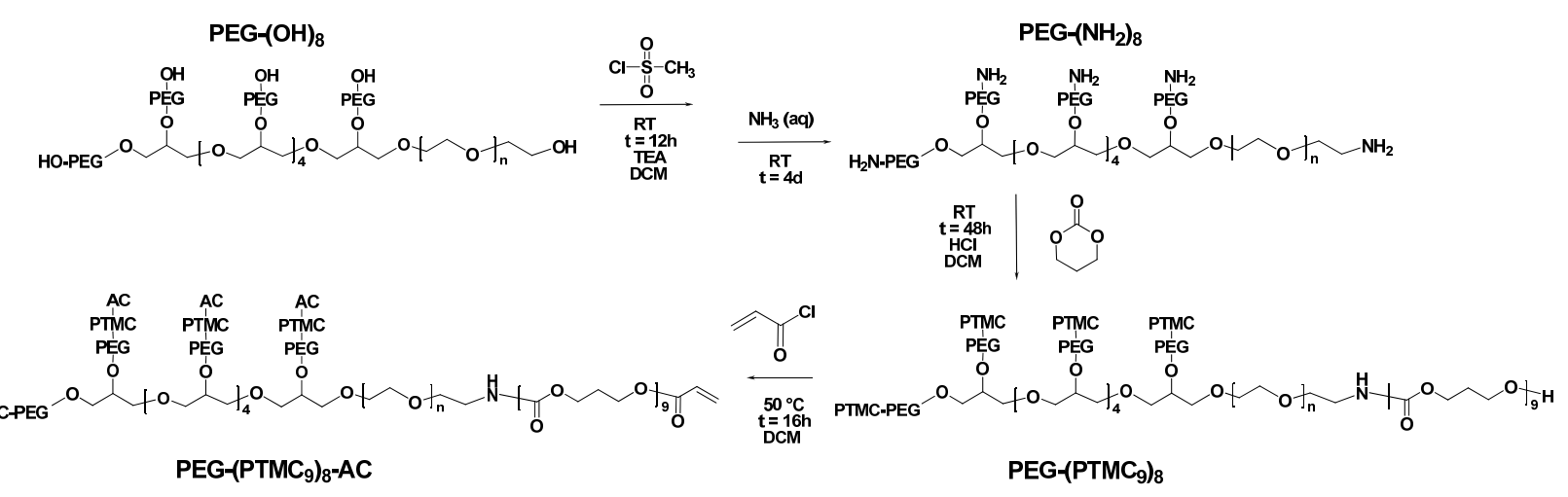

Figure 1. Synthesis scheme for the preparation of PEG-(PTMC $)_{8}$ and PEG-(PTMC $)_{8}-\mathrm{AC}_{8}$ star block copolymers.

The ${ }^{1} \mathrm{H}$ NMR spectrum of the PEG-(PTMC 9$)_{8}$ star block copolymer shows the methylene protons of the PTMC blocks at 4.22 and $2.03 \mathrm{ppm}$ (Figure 2A). The signal of the methylene protons next to the amine functional groups $\left(\mathrm{CH}_{2}-\mathrm{NH}_{2}\right)$ of the starting PEG amine completely disappeared, revealing full end group conversion. Signals of the carbamate $\mathrm{NH}$ and methylene $-\mathrm{CH}_{2}-\mathrm{NH}-\mathrm{COO}-$ protons appeared at $7.92 \mathrm{ppm}$ and $4.28 \mathrm{ppm}$, respectively. The degree of polymerization of the PTMC blocks was calculated using the integrals of peaks corresponding to the methylene protons of the TMC units and the main chain protons of PEG and was close to the ratio based on the feed composition. 

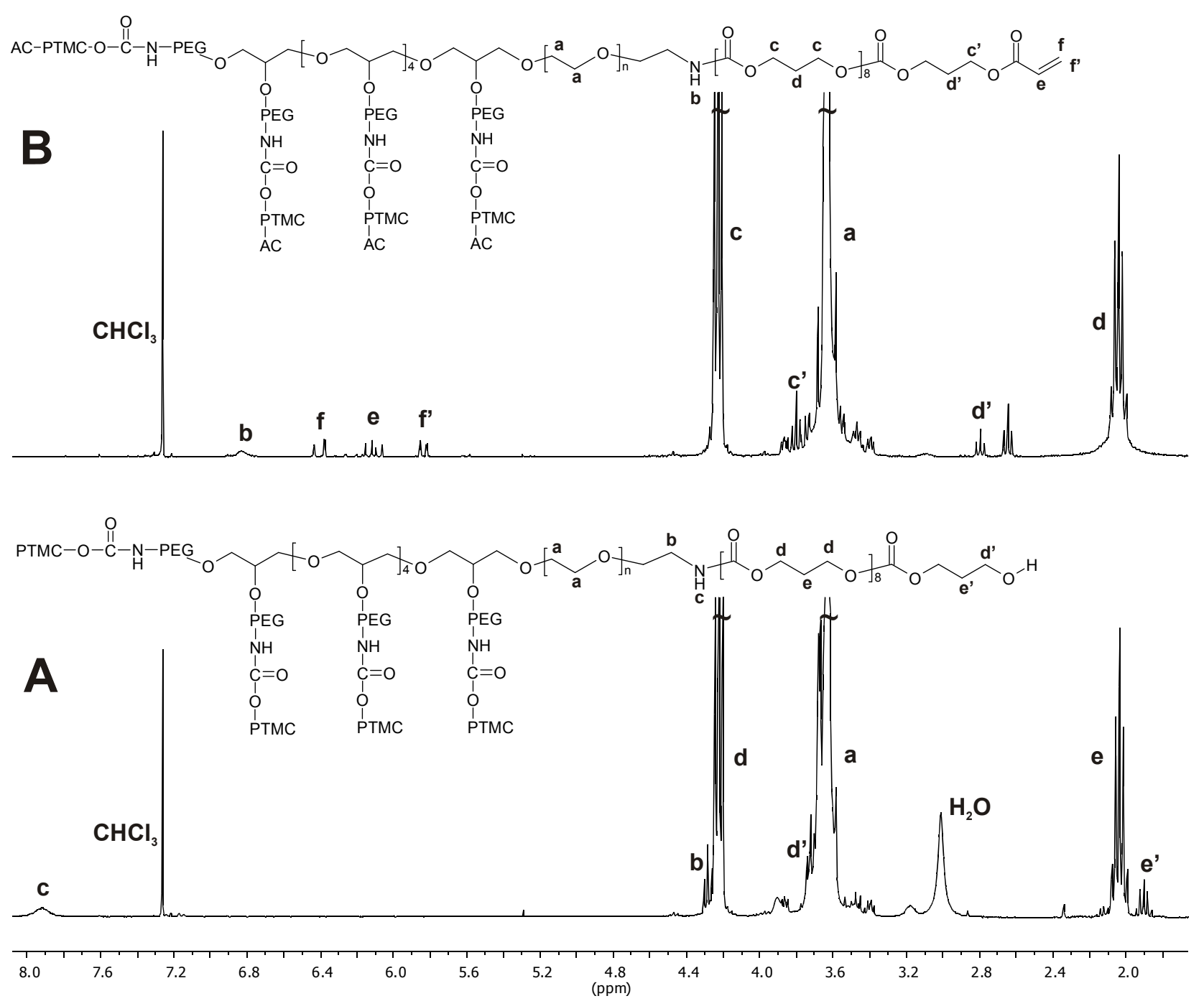

Figure 2. ${ }^{1} \mathrm{H}$ NMR spectra of PEG-(PTMC $)_{8}(\mathrm{~A})$ and PEG-(PTMC 9$)_{8}-\mathrm{AC}(\mathrm{B})$. Solvent: $\mathrm{CDCl}_{3}$.

Acrylation of PEG based block copolymers has proven an efficient way to prepare chemically crosslinked hydrogels through photopolymerization. ${ }^{19}$ The PEG-(PTMC 9$)_{8}$ star block copolymer was end group functionalized using acryloyl chloride in dichloromethane. In the ${ }^{1} \mathrm{H}$ NMR spectrum of the PEG-(PTMC 9$)_{8}$-AC new signals appeared at 6.41, 6.10 and $5.84 \mathrm{ppm}$ corresponding to the vinylic protons (Figure 2B). Furthermore, signals correlating to protons of the terminal PTMC repeating unit shifted downfield (c' and d' in Figure 2B). The degree of acrylation was calculated to be higher than $90 \%$ by comparing the respective areas of the peaks corresponding to the acryloyl groups and the PEG methylene groups.

Self-assembly of PEG-(PTMC $)_{9}$ in water. In water PEG-(PTMC $)_{8}$ star block copolymers gave turbid dispersions and no gelation was observed at room temperature even for a polymer concentration of $60 \mathrm{w} / \mathrm{v} \%$, despite the fact that they have hydrophobic and hydrophilic block lengths $\left(\mathrm{M}_{\mathrm{n}, \mathrm{PTMC}}=7400 \mathrm{~g} / \mathrm{mol}, \mathrm{M}_{\mathrm{n}}\right.$, PEG $\left.=21400 \mathrm{~g} / \mathrm{mol}\right)$ similar to those of 8-armed PEG-PLA star block copolymers $\left(\mathrm{M}_{\mathrm{n}, \text { PLA }}=6300-8600 \mathrm{~g} / \mathrm{mol}, \mathrm{M}_{\mathrm{n}}\right.$, PEG $\left.=23800 \mathrm{~g} / \mathrm{mol}\right)$ which have been observed 
to give thermo-sensitive hydrogels at relatively low concentration. ${ }^{20}$ PEG-(PTMC $)_{8}$ star block polymers with longer PTMC blocks (n up to 23) showed the same behavior.

The critical association concentration $(\mathrm{CAC})$ of the $\mathrm{PEG}-\left(\mathrm{PTMC}_{9}\right)_{8}$ star block copolymer as determined with the DPH solubilization method afforded a value of $0.004 \mathrm{w} / \mathrm{v} \%$. This value is of the same magnitude as earlier reported CAC values of multi-armed PEG-PTMC block copolymers of low molecular weight $\left(\mathrm{M}_{\mathrm{n}}\right.$, PEG $\left.=2000 \mathrm{~g} / \mathrm{mol}, \mathrm{M}_{\mathrm{n}, \text { PTMC }}=2000 \mathrm{~g} / \mathrm{mol}\right) .{ }^{8}$ However, it is two orders of magnitude lower than the CAC value $(0.19 \mathrm{w} / \mathrm{v} \%)$ of PEG-PLA star block copolymer of similar molecular weight and hydrophobic content. ${ }^{20}$ Using dynamic light scattering (DLS) the aggregate size and aggregate size distribution of PEG-(PTMC $)_{8}$ in water were investigated at different concentrations and temperatures. At concentrations of $1 \cdot 10^{-4} \mathrm{w} / \mathrm{v} \%$ up to the CAC aggregates with an average dimension of $300 \mathrm{~nm}$ were observed. At a concentration of $0.1 \mathrm{w} / \mathrm{v} \%$ a broad distribution around a mean aggregate size of $234 \mathrm{~nm}$ was found; with increasing PEG-(PTMC 9$)_{8}$ concentration the aggregate size distribution became clearly bimodal with maximum dimensions increasing, reaching $1 \mu \mathrm{m}$ at a concentration of $10 \mathrm{w} / \mathrm{v} \%$ (Figure $3 \mathrm{~A}$ ). The size of the aggregates was independent of the temperature in between 25 and $55^{\circ} \mathrm{C}$. Aggregates with average sizes on the order of a few hundred nm were reported for other 8 -armed PEG-PTMC copolymers ${ }^{8}$ and were also formed by a $\left(\mathrm{PTMC}_{10}\right)$-PEG-(PTMC 10$)$ triblock $\left(\mathrm{M}_{\mathrm{n}}\right.$ 1000-8000-1000 g/mol) copolymer (see Figure $3 \mathrm{~B})$. Immediately after mixing $(1 / 1 \mathrm{v} / \mathrm{v})$ of dispersions of $\left(\mathrm{PTMC}_{10}\right)$-PEG-(PTMC 10$)$ and PEG$\left(\mathrm{PTMC}_{9}\right)_{8}$, aggregates with a size in between the values of the two separate dispersions were detected by DLS. This indicates that the aggregates quickly reorganize, i.e. that the individual PEGPTMC molecules are able to exchange between aggregates and to form new aggregates with another dimension. These results suggest that due to the instability of the highly constrained supramolecular aggregates a new thermodynamic equilibrium is rapidly formed even at high concentrations. 

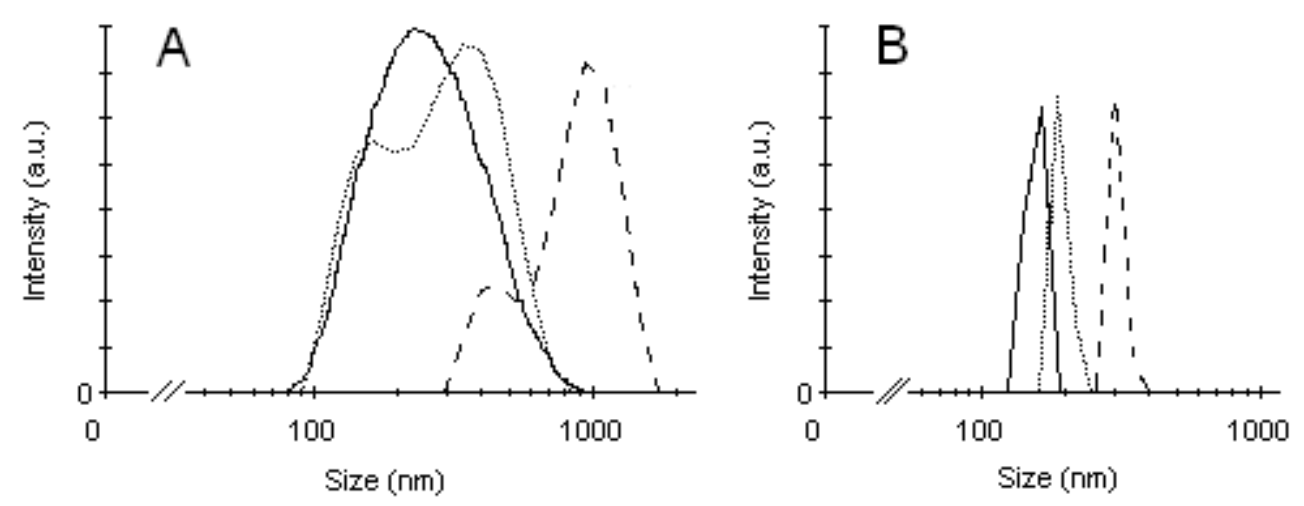

Figure 3. (A) Aggregate size distributions of PEG-(PTMC $)_{8}$ star block copolymers in water at 25 ${ }^{\circ} \mathrm{C} .0 .1 \mathrm{w} / \mathrm{v} \%$ (solid line), $1 \mathrm{w} / \mathrm{v} \%$ (dotted line) and $10 \mathrm{w} / \mathrm{v} \%$ (dashed line). (B) Aggregate size distributions of PEG-PTMC triblock and star block copolymers in water at $25{ }^{\circ} \mathrm{C} .1 \cdot 10^{-4} \mathrm{w} / \mathrm{v} \%$ $\left(\mathrm{PTMC}_{10}\right)$-PEG-(PTMC 10 ) (solid line), $1 \cdot 10^{-4} \mathrm{w} / \mathrm{v} \%$ PEG-(PTMC 9$)_{8}$ (dashed line) and their mixed $(1 / 1 \mathrm{v} / \mathrm{v})$ solution (dotted line).

The formation of aggregates of PEG-(PTMC $)_{8}$ in water was confirmed by solution state ${ }^{1} \mathrm{H}$ and ${ }^{13} \mathrm{C}$ NMR spectroscopy measurements on samples at several concentrations and temperatures. In fact, in the ${ }^{1} \mathrm{H}$ NMR spectra of 0.1 , 1 , and $10 \mathrm{w} / \mathrm{v} \%$ PEG-(PTMC 9$)_{8}$ samples in $\mathrm{D}_{2} \mathrm{O}$ at room temperature the methylene protons of PEG (peak a in Figure 4) and those of PTMC resonating at $1.91 \mathrm{ppm}$, belonging to the terminal PTMC units (peak $\mathbf{f}$ in Figure 4), were fully detected, whereas the PTMC protons resonating at 2.06 and 4.17 ppm (peaks e and $\mathbf{d}$ in Figure 4) gave signals with lower intensity than expected on the basis of stoichiometry. 

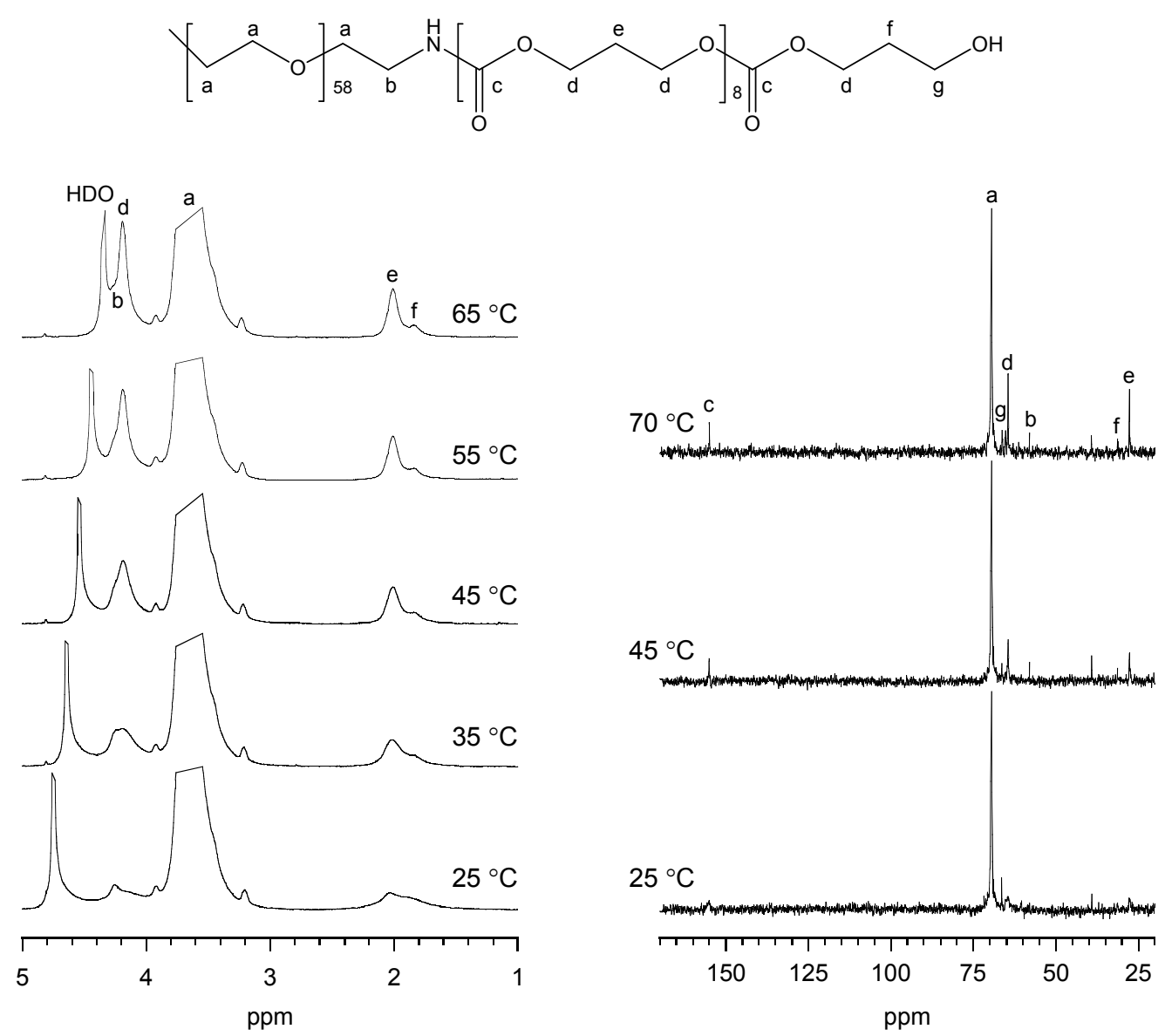

Figure 4. ${ }^{1} \mathrm{H}$ (left) and ${ }^{13} \mathrm{C}$ (right) NMR spectra of $10 \mathrm{w} / \mathrm{v} \%$ PEG-(PTMC 9$)_{8}$ in $\mathrm{D}_{2} \mathrm{O}$ at different temperatures with peak assignment. Unassigned small peaks belong to impurities.

In particular $50 \%, 66 \%$, and $74 \%$ of the latter protons were observable in the spectrum of the 10, 1 , and $0.1 \mathrm{w} / \mathrm{v} \%$ sample, respectively. Furthermore, the ${ }^{1} \mathrm{H}$ signals of PTMC were much broader than those of PEG. These observations on relative intensities and line widths of PTMC and PEG signals are similar to those reported for linear, Y shaped, and star shaped PEG-PTMC block copolymers. ${ }^{8,21-25}$ They are evidence that the PEG chains are well swollen in water and undergo fast motions, while the PTMC chains are aggregated in hydrophobic domains. The PTMC chain mobility was reduced at room temperature and increased upon heating, as highlighted by the increase in intensity and the narrowing of the PTMC ${ }^{1} \mathrm{H}$ signals with increasing temperature (Figure 4). However, while the linewidth progressively decreased with increasing temperature up to $70{ }^{\circ} \mathrm{C}$, the intensities reached a plateau value at $40{ }^{\circ} \mathrm{C}$ corresponding to $65 \%, 90 \%$ and $100 \%$ of PTMC protons for the 10,1 , and $0.1 \mathrm{w} / \mathrm{v} \%$ samples, respectively. On the other hand, the intensity and linewidth of the PEG ${ }^{1} \mathrm{H}$ signal did not change throughout the temperature range investigated. The solution state ${ }^{13} \mathrm{C}$ NMR spectra, although acquired with experimental parameters which do not guarantee quantitative results, showed temperature trends similar to those described for the ${ }^{1} \mathrm{H}$ spectra (see Figure 4). The increase of undetected PTMC units with increasing concentration suggests an increase in aggregate dimensions. 
Solid state ${ }^{13} \mathrm{C}$ NMR experiments were performed on a $50 \mathrm{w} / \mathrm{v} \%$ sample of PEG-(PTMC $)_{8}$ in $\mathrm{D}_{2} \mathrm{O}$ in order to investigate the degree of rigidity of the PTMC aggregates (Figure 5). The direct excitation (DE) spectra recorded with a short and a long recycle delay were identical, with the relative intensities of the different peaks reflecting the copolymer stoichiometry; furthermore, the cross polarization (CP) spectrum showed very little intensity. These findings concur to put in evidence a high mobility of the PTMC chains, differently from what was observed for PEG-PLA star block copolymers. ${ }^{20}$ This behavior is not surprising since it reflects the high mobility of PTMC in the solid state, as shown by the $\mathrm{DE}$ and $\mathrm{CP}$ spectra recorded on a sample of dry PEG-(PTMC 9$)_{8}$ (Figure 5). In fact, as in the hydrated sample, the DE spectra recorded with a short and a long recycle delay were identical, with peaks relative to PTMC at 28.02, 31.54, 64.74, and $155.18 \mathrm{ppm}$. On the other hand, the CP spectrum showed for PTMC only narrow peaks ascribable to a minor crystalline component, at $25.58,31.10,62.41,63.45$, and $154.62 \mathrm{ppm}$, not distinguishable in the DE spectra due to their very small intensity with respect to the overlapping broader resonances of the dominant amorphous component.

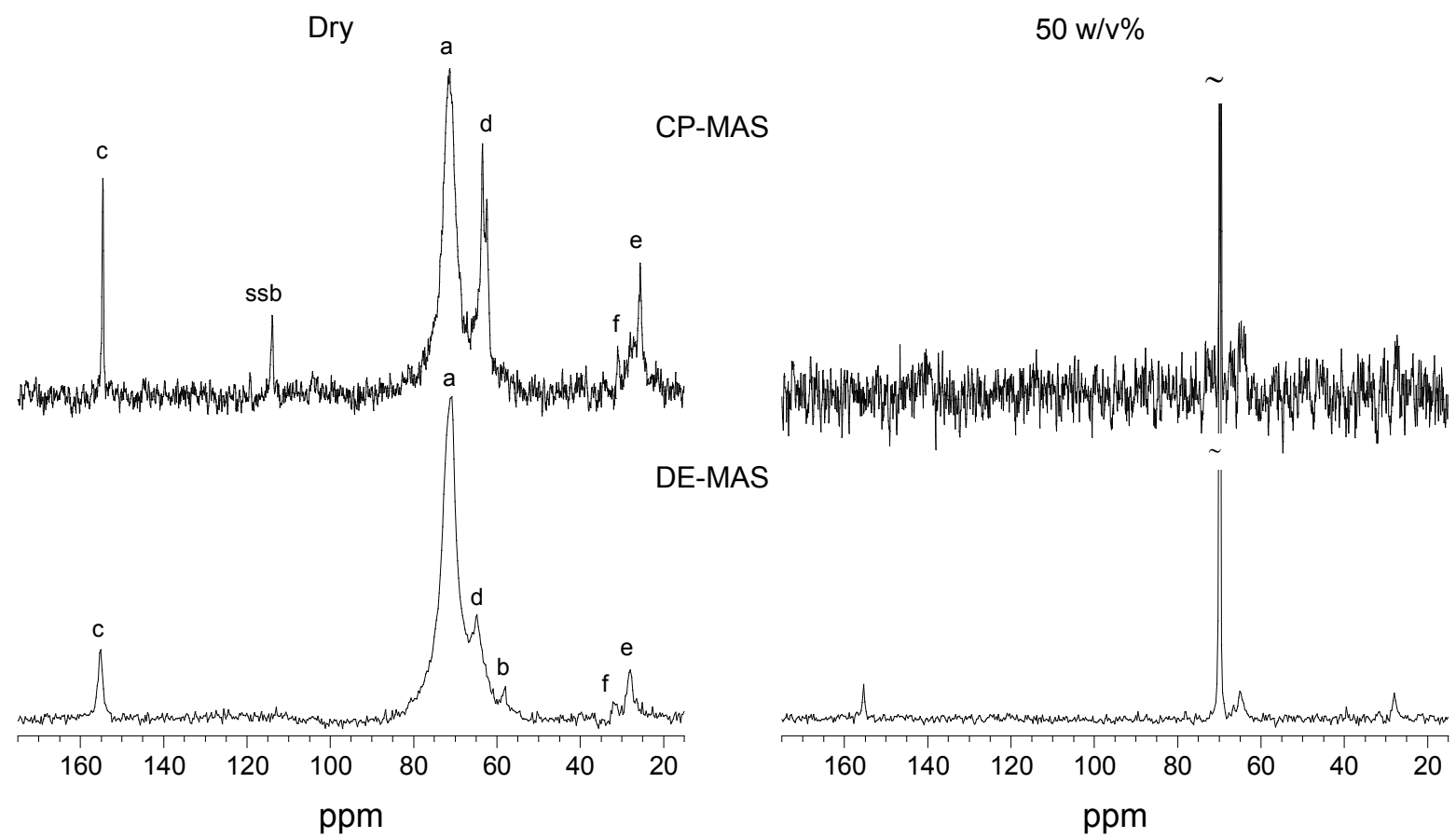

Figure 5. ${ }^{13} \mathrm{C} \mathrm{CP}$ - and DE-MAS NMR spectra of dry PEG-(PTMC 9$)_{8}$ and of a $50 \mathrm{w} / \mathrm{v} \%$ PEG$\left(\mathrm{PTMC}_{9}\right)_{8}$ sample in $\mathrm{D}_{2} \mathrm{O}$ at room temperature. $\mathrm{CP}$ and $\mathrm{DE}$ spectra were recorded acquiring 1600 and 800 scans, respectively. Letters refer to the formula in Figure 4.

The aggregation behavior of $\mathrm{PEG}-\left(\mathrm{PTMC}_{9}\right)_{8}$ in water was further investigated using oscillatory rheology. ${ }^{26}$ In Figure 6 the plateau value of $\mathrm{G}^{\prime}$, denoted as $\mathrm{G}^{\prime}{ }_{0}$, which is the value determined in the region where the storage modulus is nearly independent of frequency, is plotted as a function of concentration. For comparison, $\left(\mathrm{PTMC}_{30}\right)$-PEG-(PTMC 30$)$ triblock $\left(\mathrm{M}_{\mathrm{n}} 3000-8000-3000 \mathrm{~g} / \mathrm{mol}\right)$ and 
PEG-(PLA 13$)_{8}$ star block copolymers were investigated as well. First of all, PEG-(PTMC 9$)_{8}$ showed weak elasticity with $\mathrm{G}^{\prime}{ }_{0}$ values on the order of $10-100 \mathrm{~Pa}$, significantly lower than those found for the other systems. Moreover, a very different dependence of $\mathrm{G}^{\prime}{ }_{0}$ on concentration was found for the three copolymers. In fact, the PEG-(PLA $\left.\mathrm{PL}_{13}\right)_{8}$ star block copolymer showed a linear dependence, whereas the other copolymers showed a power law dependence of the type $\mathrm{G}_{0}{ }_{0} \sim \mathrm{C}^{a}$, with $a=5.2$ for $\left(\mathrm{PTMC}_{30}\right)$-PEG-(PTMC 30$)$ and $a=13.8$ for PEG-(PTMC 9$)_{8}$. Indeed a linear dependence of G' 0 on concentration is predicted by the transient network theory according to which the plateau modulus is given by $\mathrm{G}_{0}{ }_{0}=g N k_{\mathrm{B}} \mathrm{T}$, where $N$ is the number density of polymer chains and $g$ is a correction factor denoting the fraction of elastically active chains, which does not change with concentration. ${ }^{27}$ In the case of PEG-(PLA 13$)_{8}$ star block copolymer the application of this theory gave $g=0.13$, corresponding to one bridging chain per molecule. The power law found for the other systems suggests a different network topology in which the fraction of elastically active chains increases with concentration. Values of $a$ similar to that found for (PTMC 30$)-\mathrm{PEG}-\left(\mathrm{PTMC} \mathrm{P}_{30}\right)$ are reported in literature for ABA telechelic polymer and polyampholyte hydrogels, ${ }^{26,28}$ protein gels, $^{29}$ and poly(N-isopropylacrylamid) (PNiPAM) microgels. ${ }^{30}$ On the other hand, the value determined for PEG-(PTMC $)_{8}$ is exceptionally high. The high exponent found for these systems could be better rationalized within theories for aggregation of colloidal particles in terms of either fractals $^{31}$ or soft spheres. ${ }^{30}$

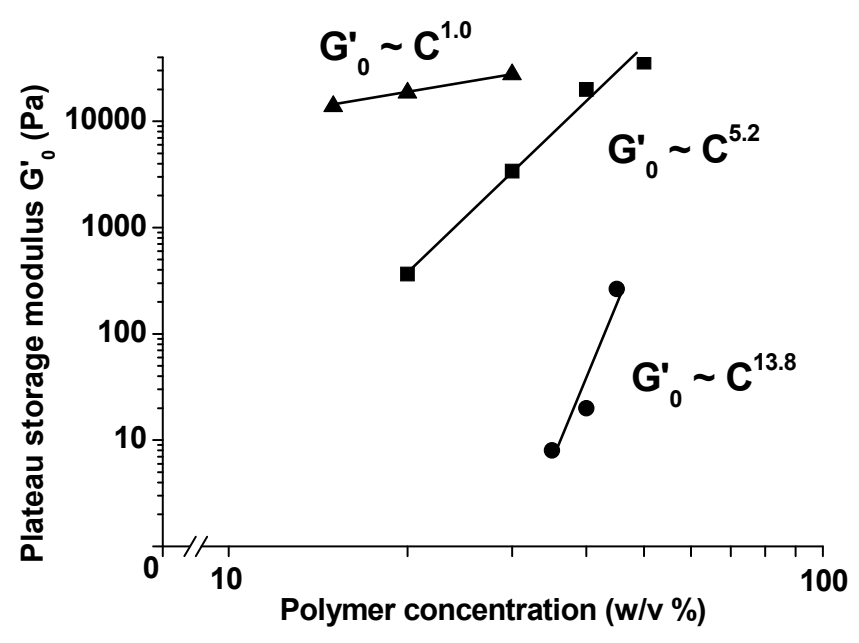

Figure 6. Plateau storage modulus $\left(\mathrm{G}_{0}^{\prime}\right)$ as a function of concentration at $25{ }^{\circ} \mathrm{C}$. PEG-(PTMC 9$)_{8}$ $(\bullet),\left(\mathrm{PTMC}_{30}\right)$-PEG-(PTMC 30$)(\boldsymbol{\bullet})$, PEG-(PLA 13$)_{8}(\boldsymbol{\Delta})$. Lines represent fitting curves.

Combining results from DLS, NMR and rheology measurements, a tentative description of the aggregation behavior of PEG-(PTMC $)_{8}$ in water can be given. On the basis of DLS findings, PEG$\left(\mathrm{PTMC}_{9}\right)_{8}$ forms aggregates with minimal dimensions of approximately $200 \mathrm{~nm}$ already well below the CAC. The aggregate size distribution broadens with increasing concentration, becoming clearly bimodal for concentrations higher than $1 \mathrm{w} / \mathrm{v} \%$. Moreover, the average size increases with 
concentration reaching micrometer dimensions (Figure 3). In contrast to the analogous 8-armed PEG-PLA star block copolymers, ${ }^{20,32}$ no aggregates with dimensions on the order of a few tens of $\mathrm{nm}$, compatible with core-shell type micelles or flower-like micelles, were detected. This result, added to the very low CAC value, is evidence of the formation at low concentration of large micelles with dimensions of approximately $200 \mathrm{~nm}$, probably resulting from secondary aggregation. ${ }^{33}$ Although with increasing concentration further aggregation of these large micelles occurs and the system becomes weakly elastic, there is no evidence of gel formation up to high concentrations $(60 \mathrm{w} / \mathrm{v} \%)$. Indeed the observed rheological properties are similar to those reported for microgel and colloid dispersions. ${ }^{30}$ The very different aggregation behavior observed for PEG$\left(\mathrm{PTMC}_{9}\right)_{8}$ with respect to PEG-(PLA $)_{8}$ can be ascribed to the differences in interactions between chains displayed by PTMC and PLA. Evidence of this was obtained from NMR experiments which clearly showed that PTMC chains are quite mobile within the aggregates also at high concentration (Figure 5) whereas PLA chains form rigid aggregates even at concentrations below the CAC. ${ }^{32}$ Possibly the close packing of micelles or aggregates, which is required for gelation, is hampered by the amorphous nature of and possibly low Van der Waals interactions between the PTMC blocks in comparison with PLA blocks.

Chemically crosslinked nanoparticles and hydrogels. When dilute aqueous PEG-(PTMC 9$)_{8^{-}}$ AC dispersions $(<5 \mathrm{w} / \mathrm{v} \%)$ were subjected to UV irradiation, covalently crosslinked nanoparticles were obtained. The average particle size, determined by DLS, yielded an average diameter of 240 nm. A scanning electron microscope (SEM) image of the nanoparticles, which have a diameter of approximately $200 \mathrm{~nm}$, is presented in Figure 7. Comparing the slightly higher value in the hydrated state $(240 \mathrm{~nm})$ to the value determined by SEM $(200 \mathrm{~nm})$ suggests that these particles do not swell considerably in water. 


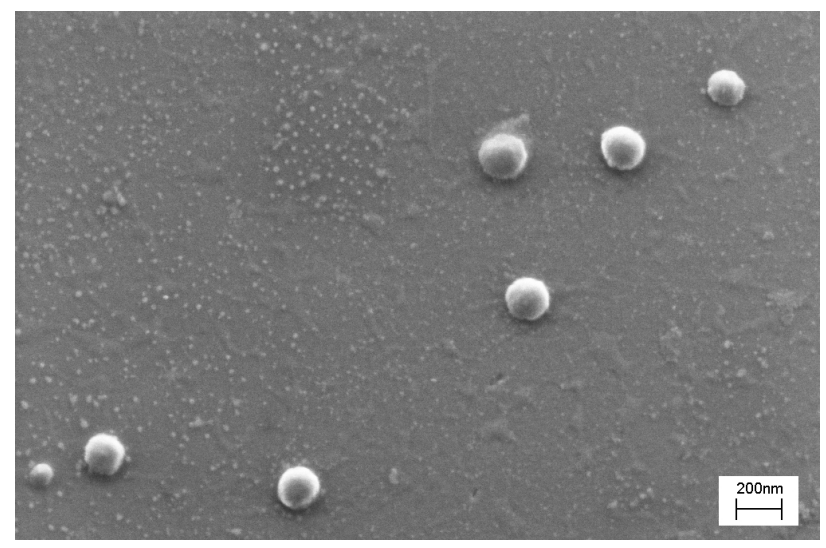

Figure 7. SEM image of PEG-PTMC nanoparticles prepared by UV irradiation of a $1 \mathrm{w} / \mathrm{v} \%$ mixture of PEG-(PTMC 9$)_{8}-\mathrm{AC}$ in water.

NMR spectroscopy was used to investigate the formation of the covalently crosslinked PEGPTMC nanoparticles in more detail. An aqueous dispersion $(1 \mathrm{w} / \mathrm{v} \%)$ of PEG-(PTMC 9$)_{8}-\mathrm{AC}_{\mathrm{Was}}$ UV irradiated in the presence of Irgacure 2959 as a photoinitiator. The nanoparticles were subsequently lyophilized and redispersed in $\mathrm{CDCl}_{3}$. The ${ }^{1} \mathrm{H}$ NMR spectrum $\left(\mathrm{CDCl}_{3}\right)$ of the covalently crosslinked PEG-PTMC nanoparticles revealed that the vinylic protons disappeared upon UV irradiation (data not shown). Furthermore, the relative intensity of the PTMC methylene signal at $4.28 \mathrm{ppm}$ decreased threefold in comparison with the starting PEG-(PTMC $)_{8}-\mathrm{AC}_{\text {star }}$ block copolymer. In aqueous environment, the hydrophobic PTMC blocks and the acrylate end groups are condensed into hydrophobic domains. Upon UV irradiation, the free radical polymerization of acrylate groups into polyacrylate chains will mainly occur in these domains. It is expected that after UV irradiation, due to the crosslinks introduced, the PTMC domains are confined to the interior of the particle even in a good solvent such as $\mathrm{CDCl}_{3}$, which results in a decreased PTMC signal intensity. The absence of any signals typical for polyacrylate chains may be explained analogously. The results show that UV irradiation of dilute aqueous PEG-(PTMC9) ${ }_{8}$-AC dispersions represents a facile method for the preparation of chemically crosslinked PEG-PTMC nanoparticles. Studies exploring the applicability of covalently crosslinked PEG-PTMC nanoparticles as drug delivery vehicles are currently in progress.

Photopolymerization of an aqueous dispersion of PEG-(PTMC 9$)_{8}$-AC at concentrations above 5 w/v \% yielded chemically crosslinked star block copolymer hydrogels. In preliminary experiments the effects of UV wavelength, reaction time and initiator concentration on the gel formation and the mechanical properties were determined. The PEG-(PTMC 9$)_{8}-\mathrm{AC}$ was dispersed in water at a concentration of $40 \mathrm{w} / \mathrm{v} \%$ and photopolymerized under various conditions. Oscillatory rheology measurements were then performed at $25^{\circ} \mathrm{C}$ by monitoring the storage $\left(G^{\prime}\right)$ and loss $\left(G^{\prime}\right.$ ') modulus of the preformed hydrogels. It followed that an initiator concentration of $10 \mathrm{~mol} \%$ relative to acrylate groups, an UV irradiation time of $120 \mathrm{~min}$ and an UV irradiation wavelength of $365 \mathrm{~nm}$ 
resulted in the highest hydrogel stiffness. The effect of the polymer concentration on hydrogel stiffness was investigated using these optimized irradiation conditions (Figure 8). The increase in G' with concentration can be ascribed to the formation of a more densely crosslinked network. These rheological experiments indicate that hydrogels can be designed with storage moduli up to 26 $\mathrm{kPa}$.

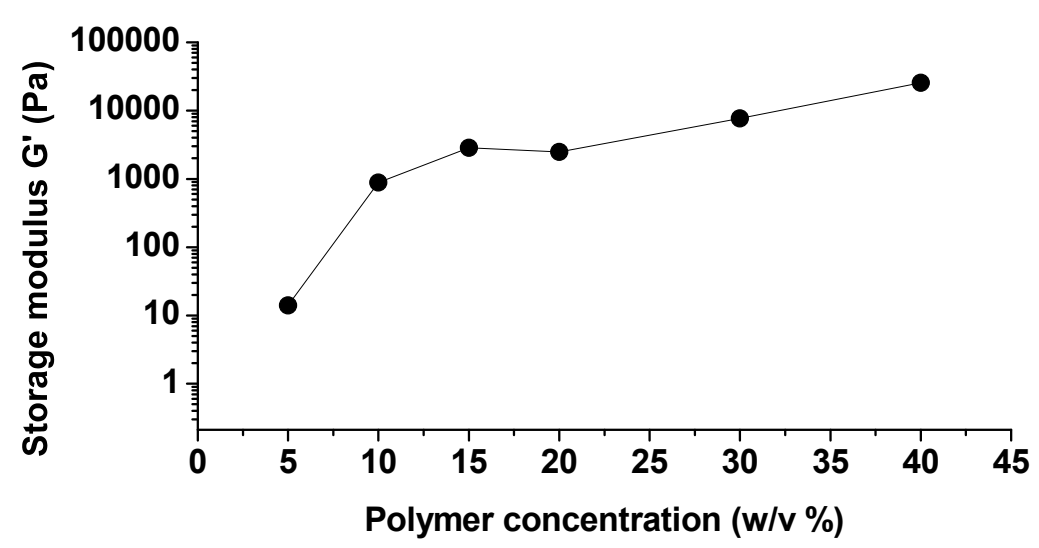

Figure 8. Storage modulus (G') of PEG-(PTMC $)_{8}-\mathrm{AC}$ hydrogels as a function of the initial polymer concentration at $25^{\circ} \mathrm{C}$.

Hydrogel degradation and cytotoxicity. The in vitro degradation of chemically crosslinked PEG-(PTMC $)_{8}$-AC hydrogels was investigated by a gravimetrical procedure. The swelling of hydrogels during degradation, as well as the polymer mass loss were monitored up to 6 weeks (Figure 9). Hydrogel samples were prepared at a $40 \mathrm{w} / \mathrm{v} \%$ polymer concentration in water and then immersed in PBS at $37{ }^{\circ} \mathrm{C}$. At regular time intervals, samples were taken out and their mass in the swollen state was determined. Subsequently, the samples were allowed to dry overnight to yield the dry polymer weight after degradation. It follows from Figure 9 that the PEG-(PTMC 9$)_{8}$-AC hydrogels possess excellent stability in PBS, showing only $10 \%$ mass loss after 6 weeks. Such a relatively slow degradation rate is commonly observed for PTMC homopolymers ${ }^{34}$ and PEG-PTMC block copolymers ${ }^{12}$ in buffer solution and can be ascribed to the high hydrolytic stability of the carbonate linkage at physiological $\mathrm{pH}$. The swelling of the hydrogels remains constant at approximately $550 \%$. The slight decrease in swelling during the first week of immersion may be due to the loss of non-crosslinked material. The observed mass loss is likely due to bulk degradation as a result of the slow hydrolysis of carbonate groups in the PTMC domains. An adequate ${ }^{1} \mathrm{H}$ NMR end group analysis could not be performed for the expected hydroxy propyl end groups coincide with the PEG signal. 


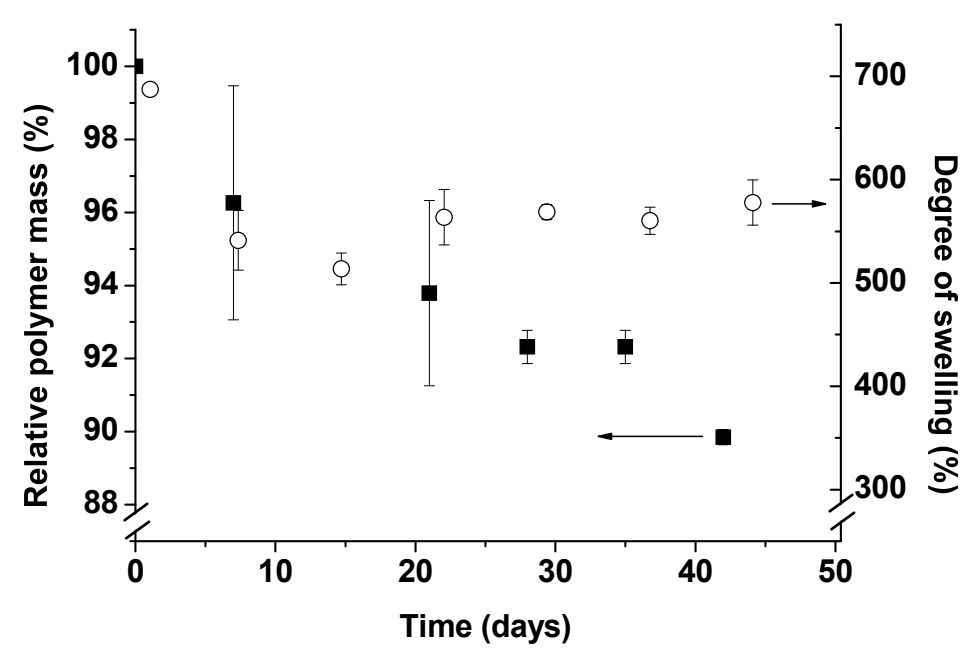

Figure 9. Relative polymer mass loss and degree of swelling of PEG-(PTMC 9$)_{8}-\mathrm{AC}_{\text {hydrogels in }}$ PBS at $37^{\circ} \mathrm{C}$.

The addition of lipase, an enzyme that has been shown to degrade PTMC polymers, ${ }^{35}$ did not significantly alter the degradation behavior of the PEG-(PTMC 9$)_{8}$-AC networks (data not shown). This is possibly due to the low molecular weight of the PTMC blocks in these PEG-(PTMC 9$)_{8}$-AC networks, as it was reported that the enzymatic degradation rate of PTMC films decreases with decreasing PTMC molecular weight. ${ }^{36}$ On water insoluble substrates, at water-lipid interfaces and on hydrophobic supports the conformation of the lipase molecule is open and the structure is hyperactive. ${ }^{37,38}$ In the absence of such an interface, however, the active center of the lipase molecules is buried under a short helical segment. ${ }^{39}$ Because of the high PEG content of the PEG$\left(\text { PTMC }_{9}\right)_{8}$-AC hydrogels, the slow enzymatic degradation may therefore also be the result of a low lipase activity.

The direct contact method was used to assess the cytotoxicity of photocrosslinked PEG$\left(\mathrm{PTMC}_{9}\right)_{8}$-AC hydrogels. The viability of bovine cartilage chondrocytes in contact with the PEG$\left(\mathrm{PTMC}_{9}\right)_{8}$-AC hydrogels for $20 \mathrm{~h}$ is similar to the viability of cells grown on a tissue culture polystyrene (TCPS) substrate (Figure 10). On the other hand, cells grown in contact with latex rubber, a positive control, exhibited a significant reduction in viability. The obtained results indicate that photocrosslinked PEG-(PTMC $)_{8}$-AC hydrogels are non-toxic to bovine cartilage chondrocytes. 


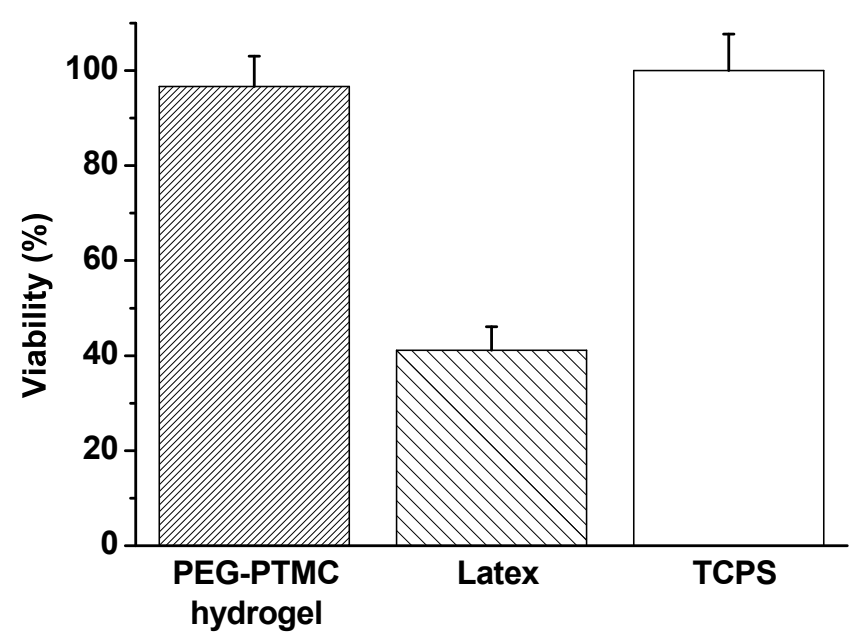

Figure 10. Viability of chondrocytes in contact with PEG-(PTMC 9$)_{8}-\mathrm{AC}$ hydrogel or latex for $20 \mathrm{~h}$. Control viability (cells grown on TCPS) was taken as $100 \%$.

\subsection{Conclusions}

A PEG-(PTMC $)_{8}$ star block copolymer was prepared by ring opening polymerization of TMC initiated by PEG-( $\left(\mathrm{NH}_{2}\right)_{8}$ in the presence of $\mathrm{HCl}$ as a catalyst. Dye solubilization experiments showed that aqueous dispersions of the copolymer form aggregates at very low concentrations and DLS experiments on PEG-(PTMC 9$)_{8}$ in water afforded aggregate sizes with a minimum dimension of $200 \mathrm{~nm}$, corresponding to large micelles resulting from secondary aggregation. Smaller micelles with dimensions on the order of a few tens of nm, present in hydrogel forming systems such as PEG-(PLA $)_{8}$, were not detected. Oscillatory rheology measurements at high concentrations showed properties similar to those reported for microgel and colloid dispersions. NMR experiments clearly showed that PTMC chains are quite mobile within the aggregates and DLS experiments revealed fast diffusion of macromolecules between aggregates. The short residence time of the PTMC blocks in the hydrophobic regions appears thus too short to obtain a stable network.

PEG-(PTMC $)_{8}$ was functionalized with acrylate end groups and photopolymerized in the presence of Irgacure 2959. UV irradiation of dilute $(<5 \mathrm{w} / \mathrm{v} \%)$ PEG-(PTMC $)_{8}-\mathrm{AC}$ dispersions resulted in covalently crosslinked PEG-PTMC nanoparticles, whereas more concentrated dispersions yielded chemically crosslinked PEG-PTMC hydrogels. Rheological experiments showed that hydrogels can be designed with storage moduli up to $26 \mathrm{kPa}$ by varying the precursor concentration and the irradiation conditions. Gravimetrical degradation experiments revealed that PEG-PTMC star block copolymer hydrogels possess excellent in vitro stability with $10 \%$ mass loss after 6 weeks. Furthermore, cytotoxicity experiments indicated that photocrosslinked PEG-PTMC hydrogels are non-toxic to chondrocytes. This study shows that photocrosslinkable PEG-(PTMC 9$)_{8-}$ AC represents a versatile system which holds promise for biomedical applications such as controlled drug delivery systems and matrices for tissue engineering. 


\subsection{Acknowledgements}

We thank M.A. Smithers from the MESA+ Institute for Nanotechnology for the SEM measurements.

\subsection{References}

[1] Peppas, N. A.; Bures, P.; Leobandung, W.; Ichikawa, H. Eur. J. Pharm. Biopharm. 2000, 50, 27-46.

[2] Drury, J. L.; Mooney, D. J. Biomaterials 2003, 24, 4337-4351.

[3] Hennink, W. E.; van Nostrum, C. F. Adv. Drug Delivery Rev. 2002, 54, 13-36.

[4] Ifkovits, J. L.; Burdick, J. A. Tissue Eng. 2007, 13, 2369-2385.

[5] Sawhney, A. S.; Pathak, C. P.; Hubbell, J. A. Macromolecules 1993, 26, 581-587.

[6] Jain, A. K.; Goyal, A. K.; Gupta, P. N.; Khatri, K.; Mishra, N.; Mehta, A.; Mangal, S.; Vyas, S. P. J. Controlled Release 2009, 136, 161-169.

[7] Matsumura, S.; Harai, S.; Toshima, K. Macromol. Rapid Commun. 2001, 22, 215-218.

[8] Kim, B. S.; Oh, J. M.; Cho, J. S.; Lee, S. H.; Lee, B.; Khang, G.; Lee, H. B.; Kim, M. S. J. Appl. Polym. Sci. 2009, 111, 1706-1712.

[9] Morinaga, H.; Ochiai, B.; Mori, H.; Endo, T. J. Polym. Sci., Part A: Polym. Chem. 2006, 44, 1985-1996.

[10] Kim, S. Y.; Kim, H. J.; Lee, K. E.; Han, S. S.; Sohn, Y. S.; Jeong, B. Macromolecules 2007, 40, 5519-5525.

[11] Bat, E.; Grijpma, D. W.; Feijen, J. J. Controlled Release 2008, 132, e37-e39.

[12] Matsuda, T.; Kwon, I. K.; Kidoaki, S. Biomacromolecules 2004, 5, 295-305.

[13] Hiemstra, C.; Zhong, Z.; Dijkstra, P. J.; Feijen, J. Macromol. Symp. 2005, 224, 119-131.

[14] Elbert, D. L.; Hubbell, J. A. Biomacromolecules 2001, 2, 430-441.

[15] Alexandridis, P.; Holzwarth, J. F.; Hatton, T. A. Macromolecules 1994, 27, 2414-2425.

[16] Geppi, M.; Forte, C. J. Magn. Reson. 1999, 137, 177-185.

[17] Risbud, M.; Saheb, D. N.; Jog, J.; Bhonde, R. Biomaterials 2001, 22, 1591-1597.

[18] Hyun, H.; Kim, M. S.; Khang, G.; Lee, H. B. J. Polym. Sci., Part A: Polym. Chem. 2006, 44, 4235-4241.

[19] Metters, A. T.; Anseth, K. S.; Bowman, C. N. Polymer 2000, 41, 3993-4004.

[20] Buwalda, S. J.; Dijkstra, P. J.; Calucci, L.; Forte, C.; Feijen, J. Biomacromolecules 2010, 11, 224-232.

[21] Kim, M. S.; Hyun, H.; Kim, B. S.; Khang, G.; Lee, H. B. Curr. Appl. Phys. 2008, 8, 646650.

[22] Zhang, H. H.; Huang, Z. Q.; Sun, B. W.; Guo, J. X.; Wang, J. L.; Chen, Y. Q. J. Polym. Sci., Part A: Polym. Chem. 2008, 46, 8131-8140.

[23] Hyun, H.; Lee, J. W.; Cho, J. S.; Kim, Y. H.; Lee, C. R.; Kim, M. S.; Khang, G.; Lee, H. B. Colloids Surf., A 2008, 313-314, 131-135.

[24] Feng, J.; Su, W.; Wang, H. F.; Huang, F. W.; Zhang, X. Z.; Zhuo, R. X. ACS Appl. Mater. Interfaces 2009, 1, 2729-2737.

[25] Cho, J. S.; Kim, B. S.; Hyun, H.; Youn, J. Y.; Kim, M. S.; Ko, J. H.; Park, Y. H.; Khang, G.; Lee, H. B. Polymer 2008, 49, 1777-1782.

[26] Stavrouli, N.; Aubry, T.; Tsitsilianis, C. Polymer 2008, 49, 1249-1256.

[27] Tsitsilianis, C.; Iliopoulos, I. Macromolecules 2002, 35, 3662-3667.

[28] Bossard, F.; Sfika, V.; Tsitsilianis, C. Macromolecules 2004, 37, 3899-3904.

[29] Renkema, J. M. S.; van Vliet, T. Food Hydrocolloids 2004, 18, 483-487.

[30] Senff, H.; Richtering, W. J. Chem. Phys. 1999, 111, 1705-1711.

[31] Chae, B. S.; Lee, Y. S.; Jhon, M. S. Colloid Polym. Sci. 2004, 282, 236-242.

[32] Calucci, L.; Forte, C.; Buwalda, S. J.; Dijkstra, P. J.; Feijen, J. Langmuir 2010, 26, 12890-12896. 
[33] Hong, H. Y.; Mai, Y. Y.; Zhou, Y. F.; Yan, D. Y.; Cui, J. Macromol. Rapid Commun. 2007, 28, 591-596.

[34] Kluin, O. S.; van der Mei, H. C.; Busscher, H. J.; Neut, D. Biomaterials 2009, 30, 47384742.

[35] Zhang, C.; Subramanian, H.; Grailer, J. J.; Tiwari, A.; Pilla, S.; Steeber, D. A.; Gong, S. Polym. Adv. Technol. 2009, 20, 742-747.

[36] Zhang, Z.; Kuijer, R.; Bulstra, S. K.; Grijpma, D. W.; Feijen, J. Biomaterials 2006, 27, 1741-1748.

[37] Winkler, F. K.; D’Arcy, A.; Hunziker, W. Nature 1990, 343, 771-774.

[38] Bastida, A.; Sabuquillo, P.; Armisen, P.; Fernandez-Lafuente, R.; Huguet, J.; Guisan, J. M. Biotechnol. Bioeng. 1998, 58, 486-493.

[39] Brady, L.; Brzozowski, A. M.; Derewenda, Z. S.; Dodson, E.; Dodson, G.; Tolley, S.; Turkenburg, J. P.; Christiansen, L.; Huge-Jensen, B.; Norskov, L.; Thim, L.; Menge, U. Nature 1990, 343, 767-770. 


\title{
Chapter 6
}

\section{In situ forming PEG-PLA hydrogels via Michael addition. Mechanical properties, degradation and protein release}

This chapter has been submitted for publication: Sytze J. Buwalda, ${ }^{a}$ Pieter J. Dijkstra, ${ }^{a}$ and Jan Feijen ${ }^{\text {a }}$ Macromolecular Bioscience 2011, submitted.

a Department of Polymer Chemistry and Biomaterials, Faculty of Science and Technology, MIRA Institute for Biomedical Technology and Technical Medicine, University of Twente, P.O. Box 217, 7500 AE Enschede, The Netherlands

\begin{abstract}
Protein release from in situ forming chemically crosslinked poly(ethylene glycol)-poly(lactide) (PEG-PLA) hydrogels was studied in relation to their crosslink density and degradation properties. Chemically crosslinked hydrogels were prepared from 8-armed PEG-PLA star block copolymers bearing acrylate end groups (PEG-(PLA $)_{8}-\mathrm{AC}$ with $\mathrm{n}=4$ or 12) and multifunctional PEG thiols (PEG-(SH) $)_{n}$ with $\mathrm{n}=2,4$ or 8 ) through a Michael type addition reaction. Oscillatory rheology measurements showed that hydrogels can be obtained within 1 minute after mixing PEG-(PLA 4$)_{8-}$ $\mathrm{AC}$ and PEG-(SH) 8 in PBS, quickly reaching a relatively high storage modulus of $16.6 \mathrm{kPa}$. Degradation times increased with crosslink density and varied from 3 days for PEG-(PLA 12$)_{8}$-AC / PEG-(SH) $)_{2}$ hydrogels to several months for PEG-(PLA 12$)_{8}-\mathrm{AC} / \mathrm{PEG}-(\mathrm{SH})_{8}$ hydrogels. The model proteins lysozyme and albumin were released over a period of 4 weeks from PEG-(PLA 12$)_{8}-\mathrm{AC} /$ PEG-(SH) $)_{8}$ hydrogels. The release of lysozyme from PEG-(PLA 12$)_{8}-\mathrm{AC} / \mathrm{PEG}-(\mathrm{SH})_{2}$ and PEG$\left(\mathrm{PLA}_{12}\right)_{8}$-AC / PEG-(SH) $)_{4}$ hydrogels was significantly faster with complete release in 3 and 12 days, respectively. Because the mesh sizes of all hydrogels are larger than the hydrodynamic diameters of the proteins the release is rate is mainly governed by diffusion, whereas for rapidly degrading hydrogels, degradation also plays a role. The PEG-PLA hydrogels, which are obtained at remarkably low macromonomer concentrations, can be tuned with a broad range of mechanical, degradation and release properties and therefore hold promise as a versatile system for the controlled delivery of biologically active agents.
\end{abstract}




\subsection{Introduction}

Hydrogels have received much interest for use in biomedical applications such as tissue engineering and the controlled delivery of biologically active agents, because they generally exhibit excellent biocompatibility due to their high water content. ${ }^{1-3}$ Hydrogels are highly water swollen polymer networks comprising either physical crosslinks, by e.g. hydrophobic or ionic interactions, or chemical crosslinks. Poly(ethylene glycol) (PEG) is a widely used hydrophilic component in hydrogels because of its good biocompatibility. As a hydrophobic component, aliphatic polyesters such as poly(lactide) (PLA) and poly( $\varepsilon$-caprolactone) (PCL) have been used extensively in block copolymers with PEG because they can be degraded in the body by hydrolysis, thus eliminating the need for explantation after their function. Hydrogels are preferably designed such that they will form in situ from precursor solutions and these systems are called injectable hydrogels. Such hydrogels offer several advantages over gel constructs pre-made before implantation. ${ }^{4}$ There is no need for surgical procedures, the initial flowing nature of the precursor solution ensures proper shape adaptation, and biological components can be incorporated in the hydrogel by simple mixing with the precursor polymer solution. Compared to in situ physically crosslinked hydrogels, chemically crosslinked hydrogels usually have better mechanical properties and are more resistant to degradation. Injectable chemically crosslinked hydrogels have been mostly prepared by photocuring of polymers functionalized with vinylic groups. ${ }^{5}$ The presence of photoinitiators and the generated radicals in the polymerization process, however, may be toxic to cells. ${ }^{6}$ Further drawbacks of photocrosslinkable systems include the limited penetration depth of UV radiation and its potentially damaging effects on living tissue. These issues prompted several researchers to study alternative pathways to prepare chemically crosslinked hydrogels like the Michael addition reaction between thiols and vinylic groups under physiological conditions. Applying this methodology in situ forming chemically crosslinked networks were prepared by reacting acrylate end group functionalized PEG with thiol group containing conjugates of natural polymers, such as chitosan, ${ }^{7}$ hyaluronic acid ${ }^{8,9}$ and dextran. ${ }^{10,11}$ In situ forming hydrogels gained much interest for the delivery of therapeutic proteins. Elbert et al. showed that by mixing aqueous solutions of a multi-armed PEG acrylate and a PEG dithiol, gelation occurred in less than 1 min at $37{ }^{\circ} \mathrm{C} .{ }^{12}$ For gels made from PEG tetra-acrylate $\left(M_{n} 15 \mathrm{~kg} / \mathrm{mol}\right)$ at a concentration of $40 \mathrm{w} / \mathrm{v} \%$ it was found that $65 \%$ of the incorporated albumin as a model protein was released with zero order kinetics over a period of $4 \mathrm{~d}$. Increasing the functionality of the PEG acrylate from 4 to 8 resulted in a slower degradation of the networks and a decreased release rate of albumin. In Michael type addition reactions vinyl sulfones are an alternative to acrylates because of their high reaction rates towards thiols. Hubbell et al. prepared various hydrogels by a Michael addition reaction between multi-armed PEG vinyl sulfones and peptides bearing thiol groups. Moreover, incorporation of protease cleavable sites allowed for enzyme-mediated degradation as well as cellular migration within these hydrogels. 
When biomolecules such as bone morphogenetic protein-2 (BMP-2) or vascular endothelial growth factor (VEGF) were incorporated, these hydrogels stimulated bone formation ${ }^{13}$ and angiogenesis in vivo, ${ }^{14}$ respectively. Zustiak et al. synthesized degradable networks by crosslinking PEG vinyl sulfones with a linear PEG dithiol containing a labile ester group. ${ }^{15}$ They showed that the degradation time could be extended from several hours to several days by increasing the molecular weight of the PEG dithiol and the number of methylene units between the ester and the thiol moieties. When the networks were modified by covalent attachment of a thiol containing peptide including the cell adhesive RGD (arginine-glycine-aspartic aid) sequence, the hydrogels supported the attachment and proliferation of fibroblasts in vitro. The use of branched PEG thiols containing a labile ester group with linear PEG acrylates in the preparation of hydrogels was described by Pritchard et al.. ${ }^{16}$ The gelation time could be tuned from a few seconds to 20 min by altering the $\mathrm{pH}$ of the solution. The hydrogels showed $15 \%$ mass loss after 9 weeks, resulting in an estimated total degradation time of 12 months. A controlled release of the steroid drug methylprednisolone was achieved for $20 \mathrm{~d}$, and the amount of drug released could be controlled by the drug load.

In this paper we report on hydrogels that are formed rapidly by a Michael type addition reaction between linear or multi-armed PEG thiols and 8-armed acrylated PEG-PLA star block copolymers. By varying the degree of branching of the PEG- $(\mathrm{SH})_{n}$ and the degree of polymerization of the PLA blocks of the acrylated PEG-PLA star block copolymers the hydrogel properties like swelling and degradation can be controlled. Importantly, the release of lysozyme and albumin as model proteins can be controlled over a broad time period. Compared to previously reported synthetic hydrogel systems crosslinked by a Michael type addition reaction, these networks are prepared using low precursor concentrations.

\subsection{Experimental section}

Materials. Hydroxyl terminated 8-armed poly(ethylene glycol) (PEG- $(\mathrm{OH})_{8}, \mathrm{M}_{\mathrm{n}}, \mathrm{NMR}=20600$ $\mathrm{g} / \mathrm{mol}$ and $21400 \mathrm{~g} / \mathrm{mol}$ ) were purchased from Jenkem (Allen, Texas, USA) and purified before use by dissolution in dichloromethane and precipitation in cold diethyl ether. PEG- $(\mathrm{OH})_{8}$ was converted in PEG-( $\left.\mathrm{NH}_{2}\right)_{8}$ following a two-step procedure analogous to that described by Elbert and Hubbell for linear hydroxyl terminated PEGs. ${ }^{17}$ L-lactide was obtained from Purac (Gorinchem, the Netherlands). Hydroxyl terminated linear poly(ethylene glycol) (PEG-(OH),$M_{n}$, NMR $=4600$ $\mathrm{g} / \mathrm{mol}$ ), acryloyl chloride, methanesulfonyl chloride (mesyl chloride), tin(II) 2-ethylhexanoate (stannous octoate), triethylamine (TEA), $25 \%$ aqueous ammonia solution, sodium hydride, allyl bromide, 2,2'-azobis(2-methylpropionitrile) (AIBN), 5,5'-dithiobis(2-nitrobenzoic acid) (Ellman's reagent), Micrococcus lysodeikticus, lysozyme from chicken egg white and albumin from bovine serum were all from Sigma-Aldrich (St Louis, Missouri, USA). Thioacetic acid and sodium thiomethoxide were purchased from Acros (Geel, Belgium). Thiol terminated 4-armed 
poly(ethylene glycol) (PEG-(SH), $\mathrm{M}_{\mathrm{n}}$, NMR $=10000 \mathrm{~g} / \mathrm{mol}$ ) was from SunBio (Orinda, California, USA). Toluene, hexane, diethyl ether, methanol and dichloromethane were all from Biosolve (Valkenswaard, the Netherlands). Dichloromethane, TEA and toluene were dried over calcium hydride, potassium hydroxide and sodium, respectively, and distilled prior to use.

Synthesis. Acrylate terminated PEG-PLA star block copolymers. The 8-armed poly(ethylene glycol)-poly(lactide) star block copolymers (PEG-(PLA) 8 ) were synthesized by ring opening polymerization of L-lactide in toluene at $110^{\circ} \mathrm{C}$. Amine terminated 8-armed star PEG (PEG$\left.\left(\mathrm{NH}_{2}\right)_{8}\right)$ and stannous octoate were used as initiator and catalyst, respectively. Acrylate end groups were introduced on PEG-(PLA) 8 by reaction with acryloyl chloride.

A typical procedure for the synthesis of a PEG-(PLA) 8 star block copolymer with a degree of polymerization (DP) of the PLA arms of 12 was as follows. To a solution of PEG-( $\left.\mathrm{NH}_{2}\right)_{8}(15 \mathrm{~g}, 0.7$ $\mathrm{mmol})$ in $75 \mathrm{ml}$ of toluene, L-lactide $(6.18 \mathrm{~g}, 42.8 \mathrm{mmol})$ and stannous octoate $(1.1 \mathrm{~g}, 2.7 \mathrm{mmol})$ were added. The reaction was allowed to proceed for $4 \mathrm{~h}$ at $110^{\circ} \mathrm{C}$ in a nitrogen atmosphere. The mixture was filtered and concentrated under reduced pressure. The product was purified by precipitation in a large excess of a cold mixture of diethyl ether and methanol $(20 / 1 \mathrm{v} / \mathrm{v})$. PEG$\left(\mathrm{PLA}_{12}\right)_{8}$ was obtained by filtration and dried overnight in vacuo at room temperature over phosphorus pentoxide. ${ }^{1} \mathrm{H}$ NMR $\left(\mathrm{CDCl}_{3}\right): \delta=6.54\left(\mathrm{~s}, \mathrm{CH}_{2} \mathrm{NHCO}\right), 5.17\left(\mathrm{~m}, \mathrm{CH}_{3} \mathrm{CH}\right), 4.35(\mathrm{q}$, $\mathrm{CH}_{3} \mathrm{CHOH}$ ), 3.64 (m, PEG protons), 2.68 (s, $\left.\mathrm{CH}_{3} \mathrm{CHOH}\right), 1.57$ (m, $\left.\mathrm{CH}_{3} \mathrm{CH}\right), 1.48$ (d, $\left.\mathrm{CH}_{3} \mathrm{CHOH}\right)$.

PEG-(PLA 12$)_{8}$ star block copolymer was functionalized with acrylate end groups (PEG-(PLA 12$)_{8}$ $\mathrm{AC})$ according to a procedure described in literature. ${ }^{18}$ PEG-(PLA $\left.{ }_{12}\right)_{8}(10 \mathrm{~g}, 0.35 \mathrm{mmol})$ was dissolved in $50 \mathrm{ml}$ of dichloromethane. Acryloyl chloride $(0.46 \mathrm{ml}, 0.6 \mathrm{mmol})$ and TEA $(0.79 \mathrm{ml}$, $0.6 \mathrm{mmol}$ ) were added and the reaction mixture was stirred under a $\mathrm{N}_{2}$ atmosphere overnight at room temperature in the presence of freshly activated molecular sieves. The reaction mixture was filtered and washed twice with brine, dried with magnesium sulfate, concentrated in vacuo and precipitated in a large excess of a cold mixture of diethyl ether and methanol $(40 / 1 \mathrm{v} / \mathrm{v})$. PEG$\left(\mathrm{PLA}_{12}\right)_{8}$-AC was obtained by filtration and dried overnight in vacuo at room temperature over phosphorus pentoxide. ${ }^{1} \mathrm{H} \mathrm{NMR}\left(\mathrm{CDCl}_{3}\right): \delta=6.53\left(\mathrm{~s}, \mathrm{CH}_{2} \mathrm{NHCO}\right), 6.48\left(\mathrm{dd}, \mathrm{OCOCHCH}_{2}\right), 6.18$ (dd, $\mathrm{OCOCHCH}$ ), 5.90 (dd, $\mathrm{OCOCHCH}_{2}$ ), 5.14 (m, $\left.\mathrm{CH}_{3} \mathrm{CH}\right), 3.64$ (PEG protons), 1.57 (d, $\mathrm{CH}_{3} \mathrm{CH}$ ), 1.48 (d, $\mathrm{CH}_{3} \mathrm{CHOCO}$ ). Acrylated PEG-(PLA) 8 star block copolymer with a DP of the PLA arms of 4 (PEG-(PLA 4$\left.)_{8}-\mathrm{AC}\right)$ was synthesized similarly.

PEG-(AC) $6 /\left(\mathrm{PLA}_{43}\right)_{2}$ star polymer, possessing 6 PEG homopolymer arms with acrylate end groups and 2 PEG-PLA 43 block copolymer arms with hydroxyl end groups, was synthesized in two steps starting from PEG-( $\left.\mathrm{NH}_{2}\right)_{8}$. In the first step, PEG-(NH$)_{8}$ was partially end group functionalized with acrylate groups to give PEG-(AC) $6 /\left(\mathrm{NH}_{2}\right)_{2}$. Briefly, PEG- $\left(\mathrm{NH}_{2}\right)_{8}(6 \mathrm{~g}, 0.29$ $\mathrm{mmol}$ ) dissolved in $60 \mathrm{ml}$ of toluene was dried by azeotropic distillation of approximately $20 \mathrm{ml}$ of 
toluene in a nitrogen atmosphere. After cooling the solution with an ice bath, $25 \mathrm{ml}$ of dichloromethane was added. Then TEA $(0.16 \mathrm{ml}, 1.2 \mathrm{mmol})$ and acryloyl chloride $(0.10 \mathrm{ml}, 1.2$ mmol) were added dropwise under stirring. The reaction mixture was allowed to warm to room temperature and was stirred for $24 \mathrm{~h}$ in a nitrogen atmosphere. The mixture was filtered, concentrated in vacuo and washed twice with brine. The product was purified by precipitation in a large excess of a cold mixture of diethyl ether and methanol $(20 / 1 \mathrm{v} / \mathrm{v})$ and after filtration dried in vacuo. Degree of acrylation: $75 \%$, yield: $88 \%$. ${ }^{1} \mathrm{H}$ NMR $\left(\mathrm{CDCl}_{3}\right): \delta=6.83\left(\mathrm{~s}, \mathrm{CH}_{2} \mathrm{NHCO}\right), 6.29$ (dd, $\left.\mathrm{OCOCHCH}_{2}\right), 6.16$ (dd, $\left.\mathrm{OCOCHCH}_{2}\right), 5.60$ (dd, $\mathrm{OCOCHCH}_{2}$ ), 3.63 (m, PEG protons), 3.17 (s, $\mathrm{CH}_{2} \mathrm{NH}_{2}$ ).

The remaining amine groups in PEG- $(\mathrm{AC})_{6} /\left(\mathrm{NH}_{2}\right)_{2}$ were subsequently used for the ring opening polymerization of lactide, catalyzed by stannous octoate. To a solution of PEG-(AC) $6 /\left(\mathrm{NH}_{2}\right)_{2}(5.3 \mathrm{~g}$, $0.25 \mathrm{mmol})$ in $10 \mathrm{ml}$ of toluene, L-lactide $(1.83 \mathrm{~g}, 12.6 \mathrm{mmol})$ and stannous octoate $(0.2 \mathrm{~g}, 0.46$ mmol) were added. The reaction was allowed to proceed for $4 \mathrm{~h}$ at $110^{\circ} \mathrm{C}$ in a nitrogen atmosphere. The mixture was filtered and concentrated under reduced pressure. PEG-(AC) $6 /\left(\mathrm{PLA}_{43}\right)_{2}$ was obtained by precipitation in a large excess of a cold mixture of diethyl ether and methanol (20/1 v/v). ${ }^{1} \mathrm{H} \mathrm{NMR}\left(\mathrm{CDCl}_{3}\right): \delta=6.83\left(\mathrm{~s}, \mathrm{CH}_{2} \mathrm{NHCO}\right), 6.29\left(\mathrm{dd}, \mathrm{OCOCHCH}_{2}\right), 6.16\left(\mathrm{dd}, \mathrm{OCOCHCH}_{2}\right)$, 5.60 (dd, $\left.\mathrm{OCOCHCH}_{2}\right), 5.16\left(\mathrm{~m}, \mathrm{CH}_{3} \mathrm{CH}\right), 4.35$ (q, $\left.\mathrm{CH}_{3} \mathrm{CHOH}\right), 3.63$ (m, PEG protons), 1.57 (m, $\left.\mathrm{CH}_{3}\right), 1.48\left(\mathrm{~d}, \mathrm{CH}_{3} \mathrm{CHOH}\right)$.

Thiol terminated PEG polymers. Thiol terminated 8-armed PEG (PEG-(SH) $)_{8}$ was synthesized starting from PEG- $(\mathrm{OH})_{8}\left(\mathrm{M}_{\mathrm{n}}\right.$, NMR $\left.=20600 \mathrm{~g} / \mathrm{mol}\right)$ according to previously described procedures. ${ }^{19,20}$ Typically, PEG- $(\mathrm{OH})_{8}(10 \mathrm{~g}, 0.49 \mathrm{mmol})$ dissolved in $350 \mathrm{ml}$ of toluene was dried by azeotropic distillation of approximately $100 \mathrm{ml}$ of toluene in a nitrogen atmosphere. Subsequently, a suspension of $\mathrm{NaH}(0.86 \mathrm{~g}, 36 \mathrm{mmol})$ in $10 \mathrm{ml}$ of toluene was added. After $30 \mathrm{~min}$ of stirring, allyl bromide $(2.7 \mathrm{ml}, 31 \mathrm{mmol})$ was added dropwise and the reaction was allowed to proceed at $110{ }^{\circ} \mathrm{C}$ overnight in a nitrogen atmosphere. After removal of the sodium salts by filtration, the reaction mixture was concentrated in vacuo and redissolved in $50 \mathrm{ml}$ of dichloromethane. The organic phase was washed 3 times with brine, dried with $\mathrm{MgSO}_{4}$ and treated with activated charcoal. The product was recovered by precipitation in a large excess of cold hexane, collected by filtration and dried in vacuo overnight. In the second step of the synthesis procedure, PEG-(allyl) $8(8.2 \mathrm{~g}, 0.39 \mathrm{mmol})$ was dissolved in $20 \mathrm{ml}$ of toluene. The solution was degassed by nitrogen bubbling for $20 \mathrm{~min}$. Subsequently AIBN (5.1 g, $0.3 \mathrm{~mol}$ ) was added, followed by the dropwise addition of thioacetic acid $(2.3 \mathrm{ml}, 32 \mathrm{mmol})$. The reaction was allowed to proceed at $65{ }^{\circ} \mathrm{C}$ for $24 \mathrm{~h}$ in a nitrogen atmosphere. The product was purified by repeated precipitations in a large excess of cold diethyl ether. PEG-(thioacetate) $)_{8}$ was obtained by filtration and dried under reduced pressure. In the final step of the synthesis procedure, PEG-(thioacetate) ${ }_{8}(5$ $\mathrm{g}, 0.23 \mathrm{mmol})$ was dissolved in $15 \mathrm{ml}$ of methanol. Sodium thiomethoxide $(0.12 \mathrm{~g}, 1.8 \mathrm{mmol})$ was 
added and the mixture was allowed to react for $30 \mathrm{~min}$ at room temperature. Subsequently $40 \mathrm{ml}$ of $0.1 \mathrm{M} \mathrm{HCl}$ was added and the resulting solution was extracted twice with dichloromethane. The combined organic phases were washed with brine, dried with $\mathrm{MgSO}_{4}$ and after evaporation of the solvent the product was purified by multiple precipitations in a large excess of cold diethyl ether. PEG-(SH) 8 was collected by filtration and dried in vacuo. Conversion: $75 \%$, yield: $52 \%$. ${ }^{1} \mathrm{H}$ NMR $\left(\mathrm{CDCl}_{3}\right): \delta=3.64$ (m, PEG protons), 2.62 (q, $\left.\mathrm{CH}_{2} \mathrm{CH}_{2} \mathrm{SH}\right) 1.87$ (q, $\left.\mathrm{CH}_{2} \mathrm{CH}_{2} \mathrm{CH}_{2} \mathrm{SH}\right) 1.38$ (t, $\left.\mathrm{CH}_{2} \mathrm{SH}\right)$.

Thiol terminated linear PEG (PEG-(SH) $)$ was prepared analogously, starting from PEG-(OH) .

PEG-PLA hydrogels. Macromonomer solutions were prepared by dissolving PEG-( $\mathrm{SH})_{\mathrm{n}}$ and PEG-(PLA $)_{8}$-AC or PEG-(AC) 6 /(PLA 43$)_{2}$ in PBS (pH 7.4) at a concentration of $5 \mathrm{w} / \mathrm{v} \%$, with thiol and acrylate end groups present in equimolar amounts. The macromonomer solutions were mixed and the Michael addition reaction was allowed to proceed for $48 \mathrm{~h}$ under gentle shaking in an inert atmosphere, resulting in the formation of a hydrogel.

Characterization. ${ }^{1} \mathrm{H}$ NMR (300 MHz) spectra were recorded on a Varian Inova $300 \mathrm{NMR}$ spectrometer. Polymers were dissolved in $\mathrm{CDCl}_{3}$ at a concentration of $15 \mathrm{mg} / \mathrm{ml}$.

Thermal properties of polymers were determined using differential scanning calorimetry (DSC). Heating and cooling rates of $20^{\circ} \mathrm{C} / \mathrm{min}$ were applied. Samples were heated from 25 to $100{ }^{\circ} \mathrm{C}$, kept at $100{ }^{\circ} \mathrm{C}$ for $1 \mathrm{~min}$, cooled to $-50{ }^{\circ} \mathrm{C}$, kept at $-50{ }^{\circ} \mathrm{C}$ for $1 \mathrm{~min}$, and finally heated to $100{ }^{\circ} \mathrm{C}$. Crystallization temperatures $\left(\mathrm{T}_{\mathrm{c}}\right)$ and corresponding enthalpies $\left(\Delta \mathrm{H}_{\mathrm{c}}\right)$ were obtained from the cooling scan, while melting temperatures $\left(\mathrm{T}_{\mathrm{m}}\right)$ and enthalpies $\left(\Delta \mathrm{H}_{\mathrm{m}}\right)$ were obtained from the second heating scan.

Ellman's reagent was used to determine the number of thiol groups in PEG-(SH) ${ }_{\mathrm{n}} \cdot{ }^{21} \mathrm{PEG}-(\mathrm{SH})_{\mathrm{n}}$ solutions were prepared by dissolving the polymer in PBS at known concentration. To a polystyrene cuvette $1 \mathrm{ml}$ of the thiol containing PBS solution and $1 \mathrm{ml}$ of a $300 \mu \mathrm{M} 5,5^{\prime}$-dithiobis(2nitrobenzoic acid) solution in PBS were added. A Cary 300 BIO UV-visible spectrometer was used to measure the absorption at a wavelength of $412 \mathrm{~nm}$. A calibration curve was constructed using 2mercaptoethanol.

Gel properties. Ellman's test was also used to determine the remaining number of thiol groups in chemically crosslinked hydrogels after $48 \mathrm{~h}$ of reaction. Freshly prepared hydrogel samples were dried in vacuo and their weight was determined. The dry networks were immersed in $2 \mathrm{ml}$ of a 150 $\mu \mathrm{M}$ 5,5'-dithiobis(2-nitrobenzoic acid) solution in PBS. After equilibration for $3 \mathrm{~h}$, the samples were analyzed as described above. 
Oscillatory rheology experiments were performed to determine the kinetics of gel formation and the mechanical properties of the chemically crosslinked hydrogels. Separate solutions (1 ml) of PEG-(SH) $)_{n}$ and PEG-(PLA $)_{8}-A C$ in PBS were mixed, homogenized and quickly applied to an Anton-Paar Physica MCR 301 rheometer. The storage modulus G' and the loss modulus G', were monitored as a function of time at $20^{\circ} \mathrm{C}$. Experiments were performed using a flat plate measuring geometry (diameter $25 \mathrm{~mm}$, gap $0.3 \mathrm{~mm}$ ) utilizing a strain of $0.5 \%$ and a frequency of $1 \mathrm{~Hz}$. To prevent water evaporation, a solvent trap was placed over the geometry.

The mesh size $\xi$ in freshly prepared hydrogels was approximated using: ${ }^{22,23}$

$$
\xi=\varphi_{\mathrm{p}}^{-\frac{1}{3}} \cdot 1 \cdot\left(\mathrm{n} \cdot \mathrm{C}_{\infty}\right)^{\frac{1}{2}}
$$

where $\varphi_{p}$ is the polymer fraction in the swollen state, 1 is the average bond length, $\mathrm{n}$ is the average number of bonds between crosslinks and $\mathrm{C}_{\infty}$ the Flory characteristic ratio. $\mathrm{C}_{\infty}$ was taken as 3.5, which is a weighted average of the values for $\mathrm{PEG}^{24}$ and PLA. ${ }^{25}$

Gravimetric degradation/dissolution experiments were performed to determine the stability of the hydrogels. Freshly prepared hydrogel samples were dried in air, their initial weight $\mathrm{W}_{0}$ was determined and samples were immersed in PBS at $37^{\circ} \mathrm{C}$. To prevent bacterial growth, $0.02 \mathrm{w} / \mathrm{v} \%$ $\mathrm{NaN}_{3}$ was added to the buffer solution. At regular times, samples were taken out and their mass in swollen state $\left(\mathrm{W}_{\mathrm{s}}\right)$ was measured after wiping the surface with tissue paper. Subsequently, the samples were allowed to dry in air overnight to yield the dry weight $\left(\mathrm{W}_{\mathrm{D}}\right)$. The degree of swelling during degradation was calculated from:

$$
\text { degree of swelling }=\frac{\left(\mathrm{W}_{\mathrm{S}}-\mathrm{W}_{\mathrm{D}}\right)}{\mathrm{W}_{\mathrm{D}}} \cdot 100 \%
$$

The remaining relative polymer mass during degradation was calculated as:

$$
\text { relative polymer mass }=\frac{\mathrm{W}_{\mathrm{D}}}{\mathrm{W}_{0}} \cdot 100 \%
$$

Chemically crosslinked hydrogels were loaded with lysozyme or albumin by dissolving lyophilized protein in a PEG-(SH) solution in PBS (pH 7.4). The loaded PEG-(SH) solution was then mixed with PEG-(PLA 12$)_{8}$-AC or PEG-(AC) $6 /\left(\mathrm{PLA}_{43}\right)_{2}$ solution in PBS in cylindrically shaped vials and the Michael addition reaction was allowed to proceed for $48 \mathrm{~h}$ in an inert atmosphere. The initial protein concentration was $10 \mathrm{wt} \%$ relative to the total polymer mass. For the in vitro release of the model proteins, $12.5 \mathrm{ml}$ of PBS was placed on top of $1.5 \mathrm{ml}$ of hydrogel and the vials were kept at $37^{\circ} \mathrm{C}$. At regular time intervals, $0.1 \mathrm{ml}$ of supernatant was removed and replaced by fresh buffer. The concentration of lysozyme and albumin in the release samples as well as the enzymatic activity of the lysozyme in selected release samples were determined as described previously. ${ }^{26}$ 


\subsection{Results and discussion}

Synthesis and characterization of acrylate- and thiol terminated macromonomers. To achieve tunable protein release rates from PEG-PLA type hydrogels, we prepared end acrylated PEG-PLA macromonomers with different DPs of the PLA blocks and thiol end functionalized PEGs. In networks based on these macromonomers degradation may occur by hydrolysis of the PLA ester or acrylate ester groups. To vary the crosslink density we prepared PEG thiols with 2, 4 or 8 arms with a constant molecular weight of $2.5 \mathrm{~kg} / \mathrm{mol}$ per arm. A PEG-(AC) $6 /\left(\mathrm{PLA}_{43}\right)_{2}$ in which the acrylate groups are attached directly to PEG via an acrylamide linkage and with two PLA blocks was used as a control. The hydrolytically stable acrylamide linkages result in networks that are significantly slower degrading.
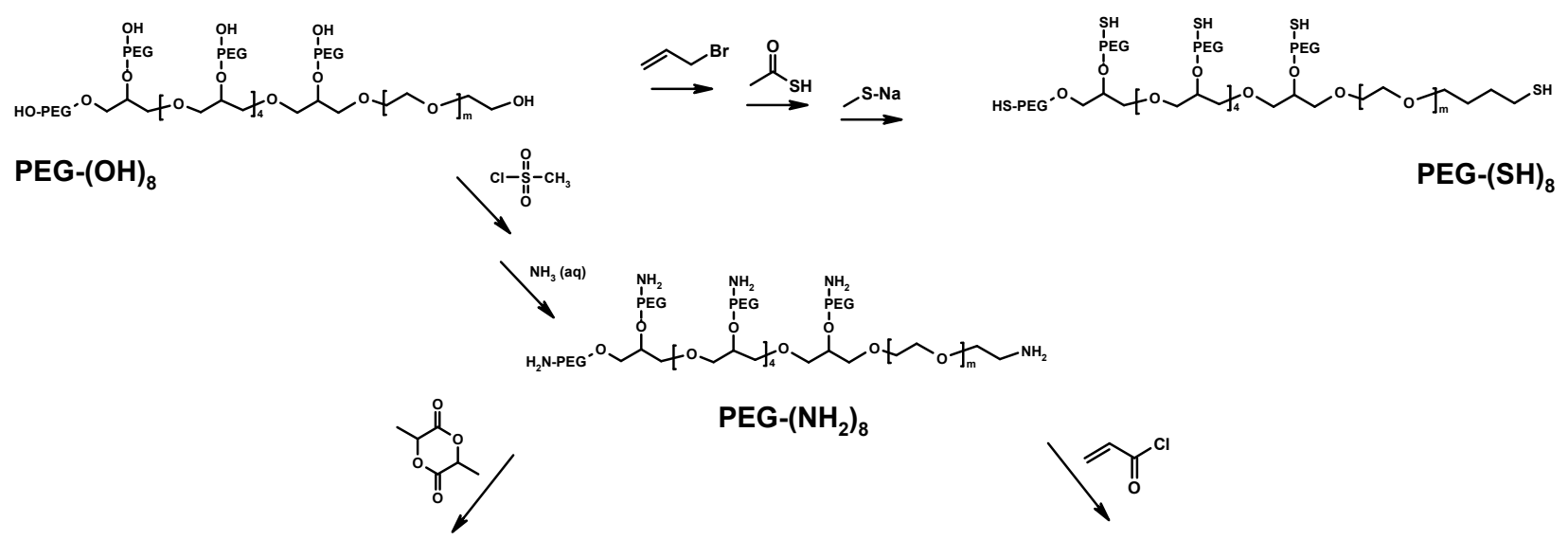

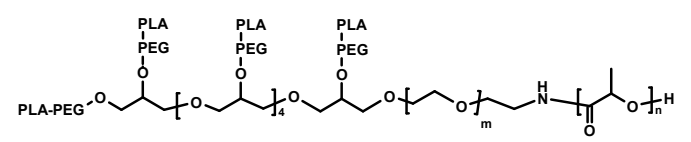

PEG-(PLA $)_{8}$
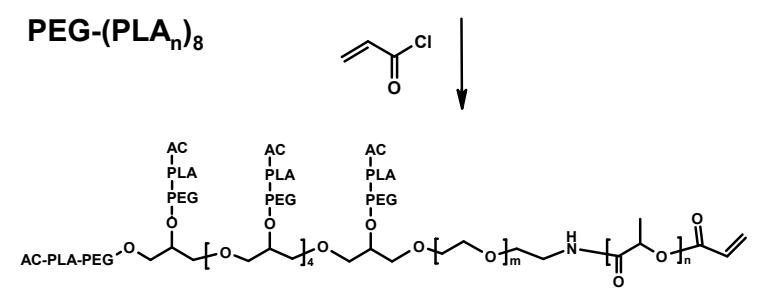

PEG-(PLA $)_{8}$-AC

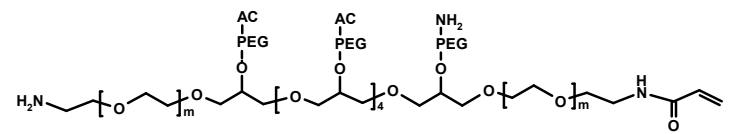

PEG-(AC) $)_{6} /\left(\mathrm{NH}_{2}\right)_{2}$
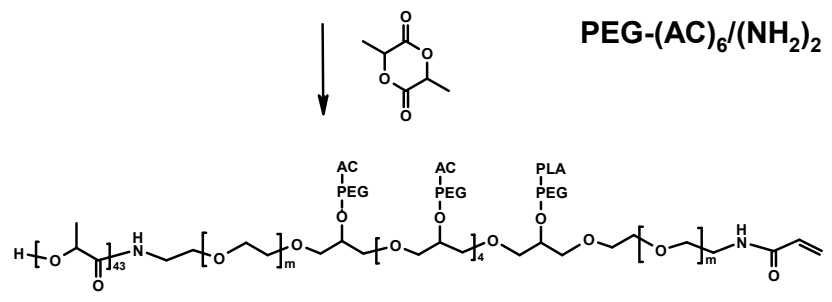

PEG-(AC) $6 /\left(\text { PLA }_{43}\right)_{2}$

Figure 1. Synthesis scheme for the preparation of PEG-(SH) $)_{8}$, PEG-(PLA $\left.{ }_{n}\right)_{8}-A C$ and PEG$(\mathrm{AC})_{6} /\left(\mathrm{PLA}_{43}\right)_{2}$ star polymers.

PEG-(PLA $)_{8}$-AC, PEG-(AC) 6 /(PLA 43$)_{2}$ and PEG-(SH) 8 star polymers were all prepared starting from PEG-(OH) 8 (Figure 1). For the synthesis of PEG-(PLA $)_{8}-A C$ and PEG-(AC) 6 (PLA 43$)_{2}$, PEG$(\mathrm{OH})_{8}$ was end group modified to PEG-( $\left.\mathrm{NH}_{2}\right)_{8}$ in high yield by converting the hydroxyl groups in their mesylate esters followed by a reaction with ammonia. ${ }^{17}$ PEG-( $\left.\mathrm{NH}_{2}\right)_{8}$ was subsequently used as an initiator in the stannous octoate catalyzed ring opening polymerization (ROP) of L-lactide in toluene at $110{ }^{\circ} \mathrm{C}$, resulting in PEG-(PLA $)_{8}$ with a hydrolytically stable amide linkage between 
PEG and PLA blocks. The average PLA block length was calculated from the ${ }^{1} \mathrm{H}$ NMR spectrum using the integrals of peaks corresponding to the methine protons of the lactyl units and the main chain protons of PEG. The isolated polymers had a PLA degree of polymerization (DP) of either 4 or 12 lactyl units depending on the monomer to initiator feed ratio. The PEG-(PLA $)_{8}$ star block copolymers were subsequently end group functionalized using acryloyl chloride. In the ${ }^{1} \mathrm{H}$ NMR spectrum of the PEG-(PLA $)_{8}$-AC signals appeared at 6.48, 6.18 and $5.90 \mathrm{ppm}$ corresponding to the vinylic protons (Figure 2, bottom). The signal at $4.35 \mathrm{ppm}$ corresponding to the terminal PLA methine protons of the starting PEG-(PLA) 8 completely disappeared, indicating quantitative end group conversion.

PEG-(AC) $6 /\left(\mathrm{PLA}_{43}\right)_{2}$ was also synthesized starting from PEG-(NH$)_{8}$ (Figure 1). First approximately $75 \%$ of the amine end groups were converted to acrylamide end groups by reaction with acryloyl chloride. Subsequently, the remaining amine groups on the partially acrylated PEG were used for the ROP of L-lactide in toluene at $110^{\circ} \mathrm{C}$ in the presence of stannous octoate as a catalyst. In the ${ }^{1} \mathrm{H}$ NMR spectrum of PEG-(AC) $6 /\left(\mathrm{PLA}_{43}\right)_{2}$ signals were present at $6.29,6.16$ and $5.60 \mathrm{ppm}$ corresponding to the vinylic protons, whereas a peak at 6.83 confirmed the presence of the acrylamide groups (data not shown). Furthermore, a signal at $4.35 \mathrm{ppm}$ was found corresponding to the terminal methine protons of hydroxyl terminated PLA chains. By rationing the respective areas of the peaks corresponding to the vinyl groups, the lactyl methine groups and the PEG methylene groups a DP of 43 lactyl units per arm was calculated. This copolymer has the same PEG to PLA weight ratio as the PEG-(PLA 12$)_{8}$-AC macromonomer. 

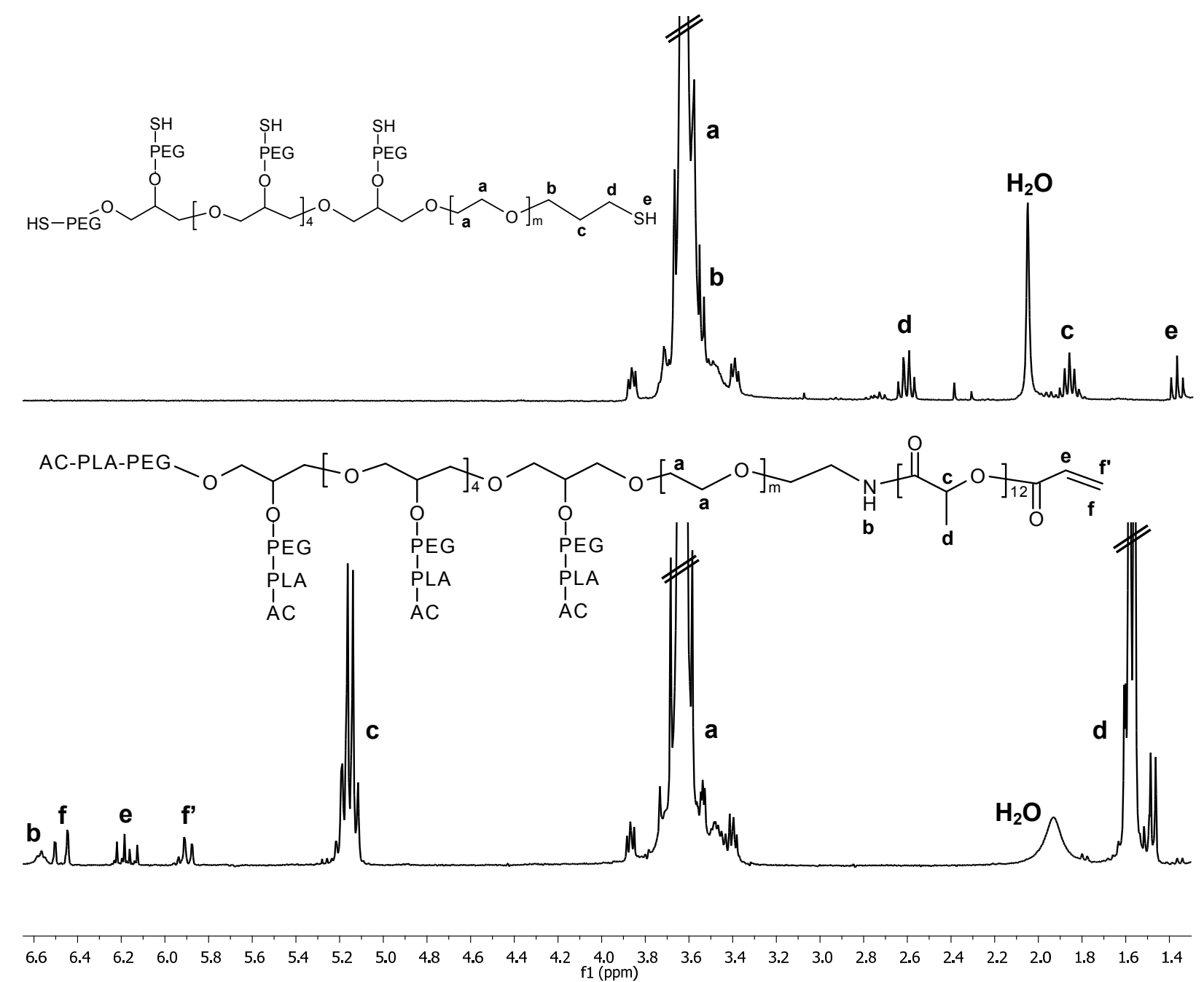

Figure 2. ${ }^{1} \mathrm{H}$ NMR spectra of PEG-(PLA 12$)_{8}-\mathrm{AC}$ (bottom) and PEG-(SH) 8 (top). Solvent: $\mathrm{CDCl}_{3}$.

PEG-(SH) $)_{\mathrm{n}}$ was synthesized starting from PEG- $(\mathrm{OH})_{\mathrm{n}} \cdot{ }^{19}$ First the PEG hydroxyl end groups were converted into allyl ether groups using allyl bromide. In the second step the PEG allyl ether was converted in PEG thioacetate by a radical mediated addition of thioacetic acid. For the conversion of the thioacetate groups in thiol groups sodium thiomethoxide was used. ${ }^{20}$ In the ${ }^{1} \mathrm{H}$ NMR spectrum of PEG-( $\mathrm{SH})_{\mathrm{n}}$ (Figure 2, top) a signal appeared at $2.61 \mathrm{ppm}$ typical for methylene protons next to thiol functional groups. No signals corresponding to protons of the starting PEG-(OH $)_{n}$ or the intermediate PEG-(allyl $)_{n}$ and PEG-(thioacetate) $)_{n}$ were observed, indicating that full end group conversion was achieved. Analysis by Ellman's reagent confirmed an almost quantitative conversion (Table 1). 
Table 1. Molecular weights and thermal properties of thiol terminated PEG macromonomers.

\begin{tabular}{|c|c|c|c|c|c|c|}
\hline \multirow[b]{2}{*}{ Polymer } & ${ }^{1} \mathrm{H}$ NMR & \multirow{2}{*}{$\begin{array}{c}\text { Degree of } \\
\text { functionalization }{ }^{a} \\
\text { (\% thiol end } \\
\text { groups })\end{array}$} & \multirow[b]{2}{*}{$\begin{array}{c}\mathrm{T}_{\mathrm{m}} \\
\left({ }^{\circ} \mathrm{C}\right)\end{array}$} & \multirow[b]{2}{*}{$\begin{array}{l}\Delta \mathrm{H}_{\mathrm{m}} \\
(\mathrm{J} / \mathrm{g})\end{array}$} & \multirow[b]{2}{*}{$\begin{array}{c}\mathrm{T}_{\mathrm{c}} \\
\left({ }^{\circ} \mathrm{C}\right)\end{array}$} & \multirow[b]{2}{*}{$\begin{array}{l}\Delta \mathrm{H}_{\mathrm{c}} \\
(\mathrm{J} / \mathrm{g})\end{array}$} \\
\hline & $\begin{array}{c}\mathrm{M}_{\mathrm{n}} \\
(\mathrm{kg} / \mathrm{mol})\end{array}$ & & & & & \\
\hline PEG-(SH) & 4.8 & 98 & 58 & 154 & 38 & 139 \\
\hline PEG-(SH) & $10.0^{b}$ & 100 & 51 & 113 & 32 & 100 \\
\hline PEG-(SH) & 21.2 & 98 & 52 & 104 & 31 & 96 \\
\hline
\end{tabular}

${ }^{a}$ Determined using Ellman's test. ${ }^{b}$ According to the manufacturer's specifications.

Table 2. Molecular weights and thermal properties of acrylated PEG-PLA macromonomers.

\begin{tabular}{|c|c|c|c|c|c|c|c|}
\hline \multirow[b]{2}{*}{ Polymer } & \multicolumn{3}{|c|}{${ }^{1} \mathrm{H}$ NMR } & \multirow[b]{2}{*}{$\begin{array}{l}\mathrm{T}_{\mathrm{m}} \\
\left({ }^{\circ} \mathrm{C}\right)\end{array}$} & \multirow[b]{2}{*}{$\begin{array}{l}\Delta \mathrm{H}_{\mathrm{m}} \\
(\mathrm{J} / \mathrm{g})\end{array}$} & \multirow[b]{2}{*}{$\begin{array}{c}\mathrm{T}_{\mathrm{c}} \\
\left({ }^{\circ} \mathrm{C}\right)\end{array}$} & \multirow[b]{2}{*}{$\begin{array}{l}\Delta \mathrm{H}_{\mathrm{c}} \\
(\mathrm{J} / \mathrm{g})\end{array}$} \\
\hline & $\mathrm{DP}^{a}$ & $\begin{array}{c}\mathrm{M}_{\mathrm{n}} \\
(\mathrm{kg} / \mathrm{mol})\end{array}$ & $\begin{array}{c}\text { Degree of } \\
\text { functionalization } \\
\text { (\% acrylate end } \\
\text { groups })\end{array}$ & & & & \\
\hline PEG-(PLA4 $)_{8}-\mathrm{AC}$ & 4.3 & 24.3 & $>95$ & 41 & 56 & 14 & 65 \\
\hline PEG-(PLA 12$)_{8}-\mathrm{AC}$ & 11.9 & 28.7 & $>95$ & 38 & 43 & 15 & 49 \\
\hline PEG-(AC) $)_{6} /\left(\mathrm{PLA}_{43}\right)_{2}$ & 43 & 27.0 & 75 & 46 & 59 & 25 & 58 \\
\hline
\end{tabular}

${ }^{a}$ Degree of polymerization of the PLA blocks, expressed in lactyl units per arm.

The PEG-PLA star block copolymers (Table 2) exhibit lower melting transitions and accompanying enthalpies than the PEG- $\left(\mathrm{NH}_{2}\right)_{8}$ precursor $\left(\mathrm{T}_{\mathrm{m}}=53{ }^{\circ} \mathrm{C}\right)$ because the crystallization of PEG is impeded by the presence of PLA blocks. ${ }^{27}$ For PEG-(AC) $)_{6} /\left(\mathrm{PLA}_{43}\right)_{2}$ a small exothermic peak was observed at $140{ }^{\circ} \mathrm{C}$ corresponding to melting of PLA domains formed by the relatively long PLA arms. The branched polymers, PEG-(SH) 4 and PEG- $(\mathrm{SH})_{8}$, exhibit lower melting temperatures and accompanying enthalpies than the linear PEG-(SH) 2 (Table 1), indicating that the PEG crystallization is hampered by the star architecture. ${ }^{28}$

Synthesis of chemically crosslinked hydrogels by a Michael addition reaction. Different combinations of macromonomer solutions of acrylated PEG-PLA and PEG-(SH) $)_{\mathrm{n}}$ in PBS (pH 7.4) were applied to prepare hydrogels via a Michael type addition reaction. The reaction was carried out at room temperature and in an inert atmosphere to prevent intra- and intermolecular disulfide bond formation due to auto-oxidation of thiols. In foregoing research it was shown that PEG$\left(\mathrm{PLA}_{12}\right)_{8}$ forms hydrogels above the critical gel concentration of $10 \mathrm{w} / \mathrm{v} \%{ }^{27}$ It was therefore decided to set the concentration of all solutions to $5 \mathrm{w} / \mathrm{v} \%$. At this concentration the hydrogels 
based on PEG-(PLA $)_{8}$-AC appeared yellow and transparent, while hydrogels based on PEG$(\mathrm{AC})_{6} /\left(\mathrm{PLA}_{43}\right)_{2}$ were almost colorless and opaque. Ellman's test ${ }^{21}$ was used to determine the amount of free thiol groups after network formation for the PEG-(PLA 12$)_{8}$-AC / PEG-(SH) 8 and PEG-(PLA 12$)_{8}$-AC / PEG-(SH) 2 hydrogels. It was found that after hydrogel formation only $1 \%$ free thiol groups remained. Rheological measurements on a $5 \mathrm{w} / \mathrm{v} \%$ solution of PEG-(SH) 8 in PBS did not show any increase in the storage modulus $G^{\prime}$ after 30 min (data not shown). This observation implies that the formation of disulfide bonds due to auto-oxidation of thiols was negligible and that gelation only occurred as a result of Michael addition between thiols and acrylates.

Hydrogel swelling. It was anticipated that the tunable hydrogel parameters designed to manipulate the protein release, i.e. the architecture of the acrylated PEG-PLA macromonomer and the number of arms of the PEG-( $\mathrm{SH})_{n}$ crosslinker, would also affect the swelling behavior and rheological properties. The degree of swelling of the networks was determined by immersing the dry networks in water for $1 \mathrm{~h}$ and measuring their weight (Table 3). Preliminary experiments confirmed that equilibrium swelling was reached within $1 \mathrm{~h}$, whereas significant hydrolytic degradation was not observed at these timescales (vide infra). Incorporation of multi-armed PEG$(\mathrm{SH})_{\mathrm{n}}$ rather than $\mathrm{PEG}-(\mathrm{SH})_{2}$ affords additional intramolecular branching points yielding networks with a higher crosslink density as well as fewer elastically inactive loops formed by the reaction of the PEG-(SH) $)_{2}$ with arms of a single PEG-PLA star molecule, ${ }^{29,30}$ resulting in a lower degree of swelling. The data in Table 3 show that not only the number of arms in PEG-(SH) but also the hydrophobicity of the acrylated PEG-PLA macromonomer can be employed to manipulate the swelling behavior of the networks. Increasing the PEG content in the networks by incorporating macromonomers with a shorter PLA block leads to a higher water uptake. Despite the similar hydrophobic/hydrophilic weight ratio as in PEG-(PLA $)_{8}-A C$, in networks based on PEG$(\mathrm{AC})_{6} /\left(\mathrm{PLA}_{43}\right)_{2}$ the location of the acrylamide and thiol groups at the ends of PEG arms in the constituent macromonomers leads to relatively large PEG domains and hence a high degree of swelling. This is contrary to networks based on PEG-(PLA $)_{8}-A C$ and PEG-(SH) in which PEG sequences between crosslinks are interrupted by PLA blocks leading to a microdomain type structure. 
Table 3. Equilibrium degree of swelling in $\mathrm{H}_{2} \mathrm{O}$ at room temperature of freshly prepared networks synthesized from thiol terminated and acrylate terminated macromonomers.

\begin{tabular}{|l|c|c|c|}
\hline $\begin{array}{c}\text { Thiol terminated macromonomers } \\
\text { Acrylate terminated macromonomers }\end{array}$ & PEG-(SH) & PEG-(SH) & PEG-(SH) \\
\hline PEG-(PLA $\left.)_{8}\right)_{8}$ AC & $610\left( \pm 40^{a}\right)$ & $520( \pm 10)$ & $490( \pm 10)$ \\
\hline PEG-(PLA $\left.{ }_{12}\right)_{8}$-AC & $540( \pm 20)$ & $410( \pm 10)$ & $370( \pm 10)$ \\
\hline PEG-(AC) ${ }_{6} /\left(\text { PLA }_{43}\right)_{2}$ & $1400( \pm 100)$ & $860( \pm 100)$ & $750( \pm 60)$ \\
\hline
\end{tabular}

${ }^{a}$ Standard deviation $(\mathrm{n}=4)$.

Rheology. When $5 \mathrm{w} / \mathrm{v} \%$ PEG-(PLA 4$)_{8}-\mathrm{AC}$ and PEG-(SH)$)_{2}$ solutions were mixed in the rheometer, the crossover point of $\mathrm{G}^{\prime}$ and $\mathrm{G}^{\prime}$, , which can be regarded as the gel point, ${ }^{31}$ was observed after $26 \mathrm{~min}$ (Figure 3, left). The storage modulus levels off after $4 \mathrm{~h}$ and eventually reaches a value of $10 \mathrm{kPa}$, after which gelation is complete. A very fast gelation occurred when 5 w/v \% PEG-(PLA 4$)_{8}$-AC and PEG-(SH) $)_{8}$ solutions were mixed. G' crossed G' after 1 min, showing that a gel is formed (Figure 3, right) and the gel stiffness continues to increase rapidly. After $20 \mathrm{~min}$ the storage modulus reaches a high value of $16.6 \mathrm{kPa}$. These data show that the mechanical properties can be modulated, similar to the swelling behavior, by manipulating the hydrogel crosslink density. The gelation times of the PEG-(PLA 4$)_{8}-\mathrm{AC} / \mathrm{PEG}-(\mathrm{SH})_{\mathrm{n}}$ networks are similar to those found for 4-armed PEG-PLA acrylates crosslinked with dithiothreitol. ${ }^{32}$ It should be noted that these materials were crosslinked at concentrations ranging from 10 to $40 \mathrm{w} / \mathrm{v} \%$, whereas the PEG-(PLA $)_{8}$-AC / PEG-(SH) networks were crosslinked at a very low concentration of $5 \mathrm{w} / \mathrm{v}$ $\%$. The results indicate that networks with excellent mechanical properties can be obtained rapidly by simple mixing of macromonomer solutions in PBS. Both the gelation times and the stiffness of the hydrogels can be tuned within a broad range.
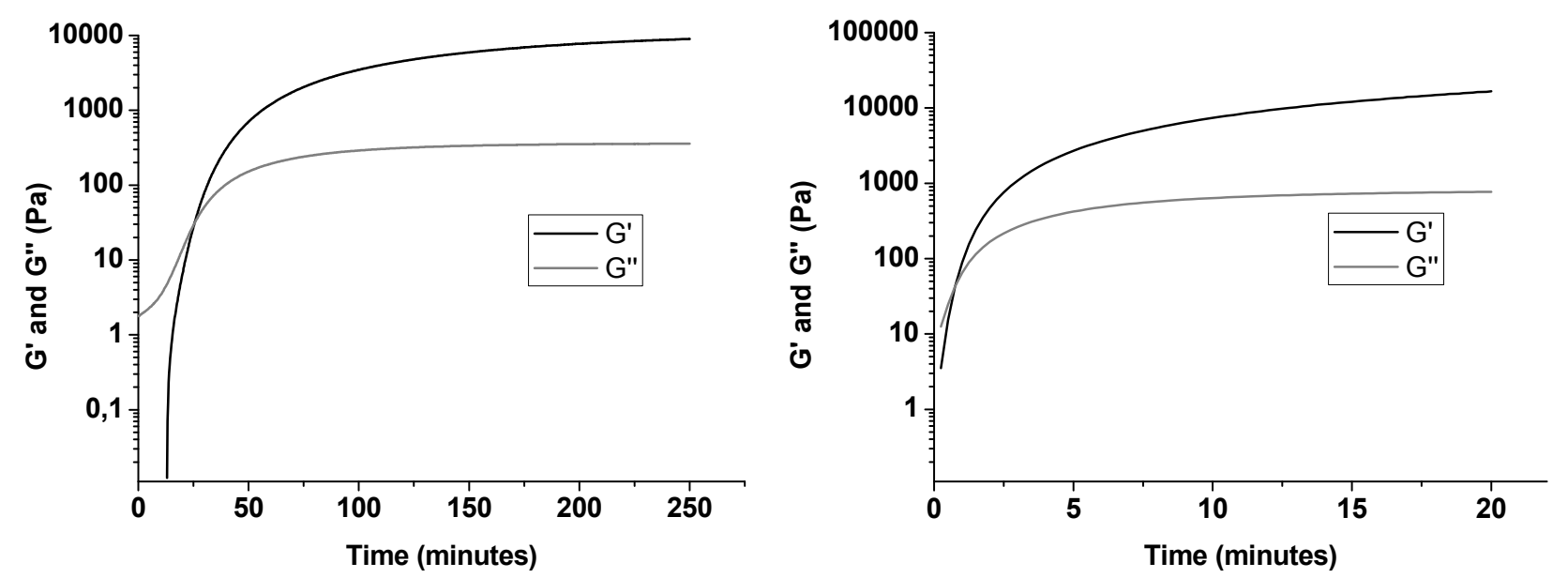

Figure 3. Storage modulus $\left(G^{\prime}\right)$ and loss modulus $\left(G^{\prime \prime}\right)$ as a function of time after mixing separate acrylate terminated and thiol terminated macromonomer PBS solutions at $20^{\circ} \mathrm{C}$. PEG-(PLA 4$)_{8}-\mathrm{AC}$ + PEG-(SH) 2 (left), PEG-(PLA 4$)_{8}-\mathrm{AC}+$ PEG-(SH) 8 (right). 
Hydrogel degradation. All PEG-(PLA $\left.{ }_{12}\right)_{8}-\mathrm{AC} / \mathrm{PEG}-(\mathrm{SH})_{\mathrm{n}}$ hydrogels were degradable under physiological conditions. Figure 4 (left) shows that PEG-(PLA 12$)_{8}-\mathrm{AC} / \mathrm{PEG}-(\mathrm{SH})_{8}$ networks loose approximately $30 \%$ of polymer mass after $30 \mathrm{~d}$ in PBS at $37^{\circ} \mathrm{C}$. The degradation of the networks is accompanied by an increase in swelling (Figure 4, right).
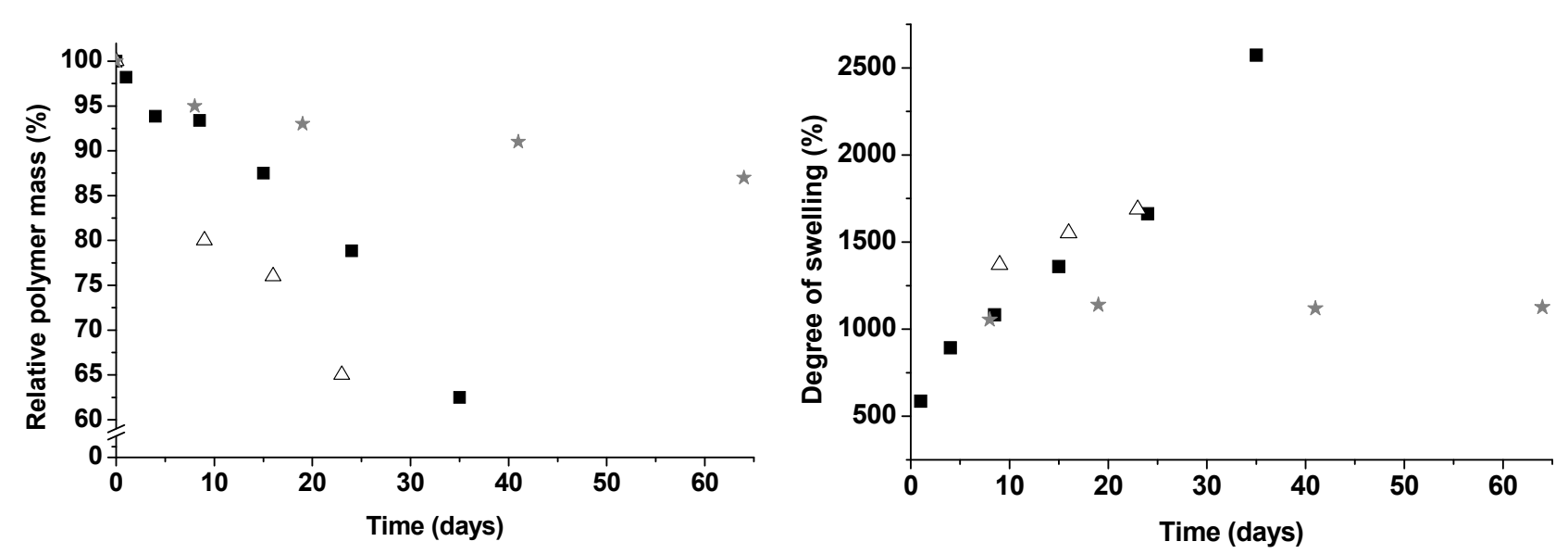

Figure 4. Polymer mass (left) and degree of swelling (right) as a function of time for PEG-(PLA 4$)_{8}{ }^{-}$ AC / PEG-(SH) 8 networks $(\triangle)$, PEG-(PLA 12$)_{8}$-AC / PEG-(SH) 8 networks (ם) and PEG$(\mathrm{AC})_{6} /\left(\mathrm{PLA}_{43}\right)_{2} / \mathrm{PEG}-(\mathrm{SH})_{8}$ networks $(\star)$ in $\mathrm{PBS}$ at $37{ }^{\circ} \mathrm{C}$. The data points represent average values of two measurements.

The degradation is likely due to predominant hydrolysis of ester bonds in the PLA chains upon which chemical crosslinks are lost, resulting in a less crosslinked hydrogel which will eventually dissolve. The PEG-(PLA 4$)_{8}-\mathrm{AC} / \mathrm{PEG}-(\mathrm{SH})_{8}$ networks exhibit a faster degradation (Figure 4, left), possibly due to a higher solvent uptake (Table 3). As anticipated, the functionality of the PEG$(\mathrm{SH})_{\mathrm{n}}$ used in the crosslinking reaction gives control over the degradation rate of the networks. Networks based on PEG-(PLA $\left.{ }_{12}\right)_{8}-\mathrm{AC}$ and PEG-(SH) $)_{4}$ or PEG- $(\mathrm{SH})_{2}$ showed a fast degradation with complete dissolution in approximately 2 weeks and $3 \mathrm{~d}$, respectively. Because these networks quickly became mechanically too weak to be handled, their degradation time was determined visually. In these networks fewer bonds need to be broken to decrease the number of chemical crosslinks in the gel. Especially the occurrence of loops in the networks based on PEG-(SH) 2 (vide supra) can be a reason for the very fast degradation of these materials. By tailoring the functionality of the macromonomers the degradation time can thus be varied from 3 days to several months.

As expected, the networks based on PEG-(AC) $6 /\left(\mathrm{PLA}_{43}\right)_{2}$ and PEG-(SH) 8 show a significantly higher stability compared to PEG-(PLA $)_{8}$-AC type networks with $13 \%$ mass loss after 9 weeks. The degree of swelling remains constant (Figure 4, right), which indicates that no chemical crosslinks are lost during the experiments. This shows that the acrylamide groups between crosslinks are not affected, despite the proximity of the electron-withdrawing thioether group which has been shown to increase the hydrolysis rate of ester groups significantly. ${ }^{33}$ The mass loss 
observed may be attributed to hydrolysis of ester groups in the PLA arms, which can be regarded as dangling chains and do not form an integral part of the network structure. The mass loss probably continues until all PLA is degraded, which would correspond to $22 \%$ of the total polymer mass.

Release of model proteins. The proteins lysozyme and albumin could be easily loaded in the hydrogels by dissolving them in the PEG-(SH) $)_{\mathrm{n}}$ solution (PBS) prior to crosslinking. Lysozyme is released from PEG-(PLA 12$)_{8}$-AC / PEG-(SH) $)_{8}$ hydrogels for $18 \mathrm{~d}$ (Figure 5, left), after which the release levels off. Albumin follows a similar release profile, but its release is slightly delayed. The release of lysozyme and albumin is approximately proportional to the square root of time up to a cumulative release of $80 \%$ and $60 \%$, respectively (Figure 5, right), indicating that the release is close to first order from this system until day 12. Calculation of the mesh size (Equation 1) of an equilibrium swollen PEG-(PLA 12$)_{8}$-AC / PEG-(SH) hydrogel affords a value of approximately 10 $\mathrm{nm}$, which is substantially larger than the hydrodynamic diameters of lysozyme and albumin (4.1 and $7.6 \mathrm{~nm}$, respectively). These results show that the release of lysozyme and albumin is diffusion controlled during the first $12 \mathrm{~d}$. The release remains approximately first order during this period despite a $10 \mathrm{w} / \mathrm{v} \%$ mass loss and consequent increased swelling due to PLA degradation (Figure 4). After $27 \mathrm{~d}$, neither lysozyme nor albumin was completely retrieved. The non-complete release may be due to reaction of some of the amine groups of the proteins with the acrylate groups of the macromonomers, leading to incorporation of the protein in the network structure by covalent bonds. ${ }^{34}$ Importantly, the released lysozyme retained $100 \%$ activity as determined by bacteria lysis experiments.
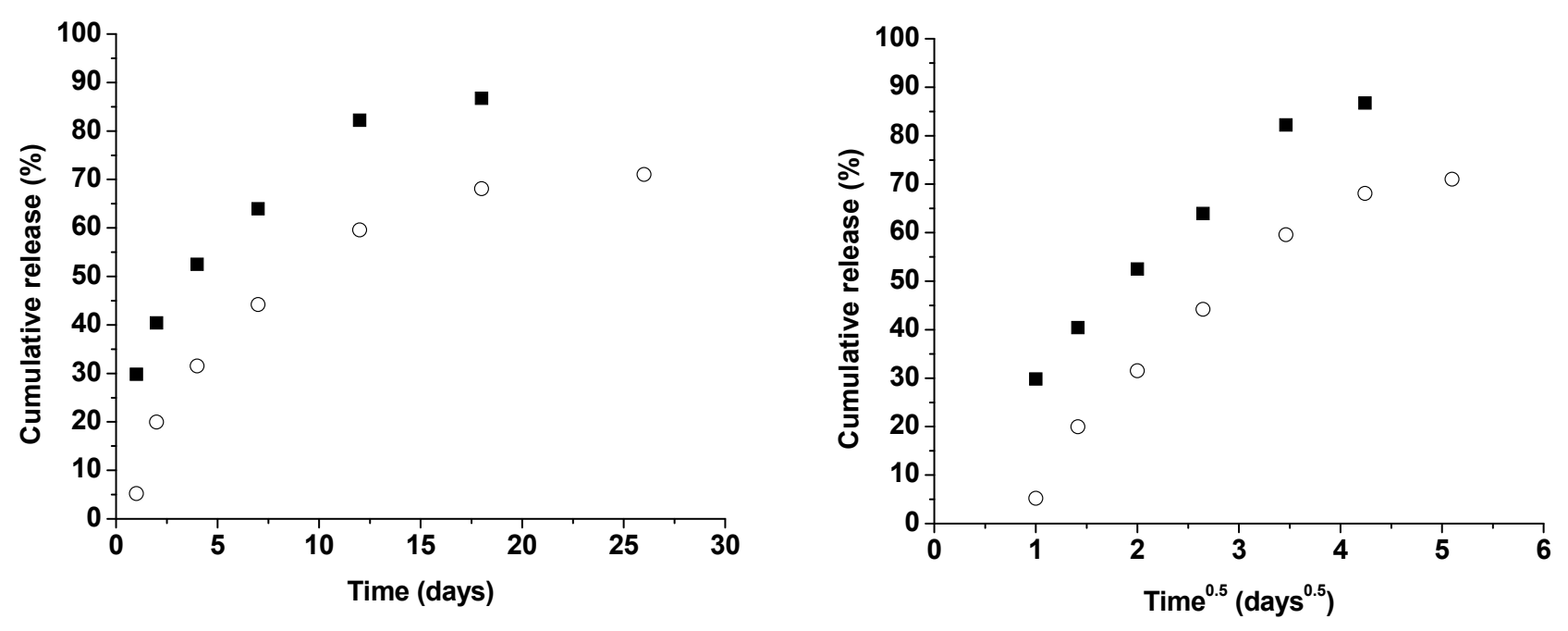

Figure 5. In vitro release of lysozyme ( $\mathbf{\square})$ and albumin $(\bigcirc)$ from PEG-(PLA 12$)_{8}-\mathrm{AC} / \mathrm{PEG}-(\mathrm{SH})_{8}$ networks. Cumulative release as a function of time (left); cumulative release as a function of the square root of time (right). 


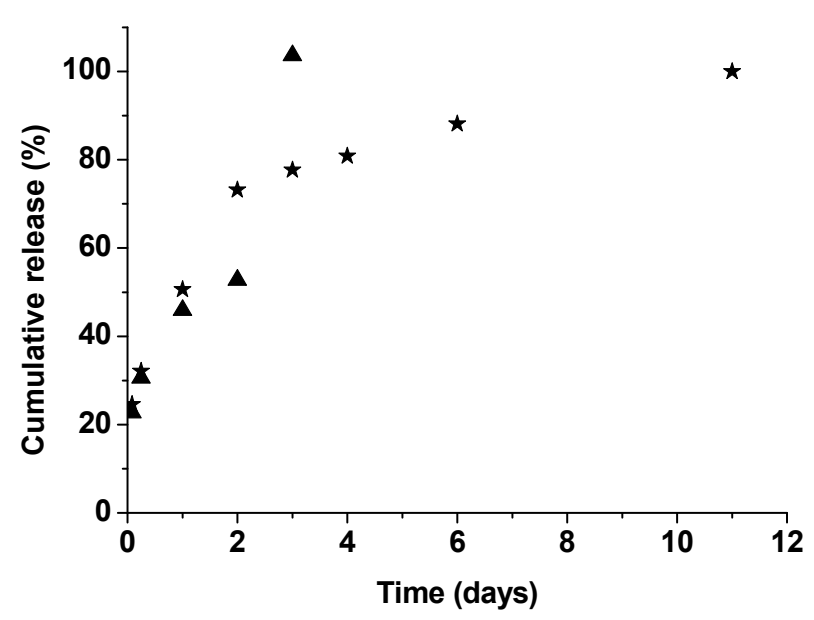

Figure 6. In vitro release of lysozyme from PEG-(PLA $\left.{ }_{12}\right)_{8}-\mathrm{AC} / \mathrm{PEG}-(\mathrm{SH})_{2}(\boldsymbol{\Delta})$ and PEG$\left(\mathrm{PLA}_{12}\right)_{8}-\mathrm{AC} / \mathrm{PEG}-(\mathrm{SH})_{4}(\star)$ networks.

Compared to PEG-(PLA 12$)_{8}$-AC / PEG-(SH) $)_{8}$ networks, hydrogels prepared using PEG-(SH) $)_{2}$ or PEG-(SH) $)_{4}$ exhibit a lower crosslink density and a faster degradation. As anticipated the release of lysozyme from PEG-(PLA 12$)_{8}$-AC / PEG-(SH) $)_{2}$ and PEG-(PLA 12$)_{8}$-AC / PEG-(SH) $)_{4}$ networks is significantly faster and the release was complete within 3 and $12 \mathrm{~d}$, respectively (Figure 6). The mesh sizes of the PEG-(PLA 12$)_{8}$-AC / PEG-(SH) $)_{2}$ and PEG-(PLA 12$)_{8}-\mathrm{AC} / \mathrm{PEG}-(\mathrm{SH})_{4}$ networks were approximately $16 \mathrm{~nm}$ and $10 \mathrm{~nm}$, respectively, but these values likely increase rapidly during the in vitro experiments. Hydrolytic degradation of the ester bonds in the PLA arms causes removal of chemical crosslinks, leading to increased swelling and eventual complete dissolution of the hydrogel at day 3 and day 12. The release from these networks is likely to be governed by a combination of diffusion and degradation.

PEG-(AC) 6 /(PLA 43$)_{2} /$ PEG-(SH) 8 networks show an expected very slow degradation (Figure 4), allowing lysozyme to be released only by diffusion through the hydrogel mesh (initial size $11 \mathrm{~nm}$ ). Release of lysozyme from these networks followed a profile comparable to that of PEG-(PLA 12$)_{8}{ }^{-}$ $\mathrm{AC} / \mathrm{PEG}-(\mathrm{SH})_{8}$ networks (data not shown). The similarity between the two release profiles confirms that the release from the PEG-(PLA 12$)_{8}-\mathrm{AC} / \mathrm{PEG}-(\mathrm{SH})_{8}$ networks (Figure 5) is controlled by diffusion only.

The results indicate that the release of the investigated proteins is mainly dependent on the crosslink density of the gels which can be tailored by the functionality of the PEG-(SH) $)_{n}$ used for gel preparation.

\subsection{Conclusions}

Chemically crosslinked PEG-PLA hydrogels were prepared by a Michael addition reaction between multi-armed PEG-(SH) and acrylated PEG-PLA star block copolymers. Several hydrogel properties could be controlled by altering the functionality of the PEG-( $\mathrm{SH})_{\mathrm{n}}$ crosslinker. Hydrogels 
were formed rapidly by simple mixing of macromonomer solutions in PBS at low concentrations ( $\sim 5 \mathrm{w} / \mathrm{v} \%$ ). Rheology showed that the hydrogels prepared from PEG-(PLA 12$)_{8}$-AC and PEG-(SH) 8 possess excellent mechanical properties which evolve quickly after mixing. The degradation of the hydrogels, ranging from a few days to several months depending on the incorporated PEG-(SH) had a pronounced effect on the protein release behavior. Lysozyme was released from the highly crosslinked PEG-(PLA 12$)_{8}$-AC / PEG-(SH) 8 hydrogels mainly by diffusion. The release of lysozyme from the PEG-(PLA 12$)_{8}$-AC / PEG-(SH) $)_{2}$ and PEG-(PLA 12$)_{8}$-AC / PEG-(SH) $)_{4}$ hydrogels was significantly faster resulting from a combination of diffusion and degradation. Importantly, the enzymatic activity of the released lysozyme was fully maintained. The possibility to be formed in situ, the robust mechanical properties and the tunable degradation and release properties make these systems very appealing as controlled drug delivery systems.

\subsection{References}

[1] Peppas, N. A.; Bures, P.; Leobandung, W.; Ichikawa, H. Eur. J. Pharm. Biopharm. 2000, 50,27-46.

[2] Drury, J. L.; Mooney, D. J. Biomaterials 2003, 24, 4337-4351.

[3] Hennink, W. E.; van Nostrum, C. F. Adv. Drug Delivery Rev. 2002, 54, 13-36.

[4] Ruel-Gariepy, E.; Leroux, J. C. Eur. J. Pharm. Biopharm. 2004, 58, 409-426.

[5] Ifkovits, J. L.; Burdick, J. A. Tissue Eng. 2007, 13, 2369-2385.

[6] Nicodemus, G. D.; Bryant, S. J. Tissue Eng., Part B 2008, 14, 149-165.

[7] Teng, D. Y.; Wu, Z. M.; Zhang, X. G.; Wang, Y. X.; Zheng, C.; Wang, Z.; Li, C. X. Polymer 2010, 51, 639-646.

[8] Censi, R.; Fieten, P. J.; di Martino, P.; Hennink, W. E.; Vermonden, T. Macromolecules 2010, 43, 5771-5778.

[9] Jin, R.; Teixeira, L. S. M.; Krouwels, A.; Dijkstra, P. J.; van Blitterswijk, C. A.; Karperien, M.; Feijen, J. Acta Biomater. 2010, 6, 1968-1977.

[10] Hiemstra, C.; van der Aa, L. J.; Zhong, Z. Y.; Dijkstra, P. J.; Feijen, J. Biomacromolecules 2007, 8, 1548-1556.

[11] Hiemstra, C.; van der Aa, L. J.; Zhong, Z. Y.; Dijkstra, P. J.; Feijen, J. Macromolecules 2007, 40, 1165-1173.

[12] Elbert, D. L.; Pratt, A. B.; Lutolf, M. P.; Halstenberg, S.; Hubbell, J. A. J. Controlled Release 2001, 76, 11-25.

[13] Lutolf, M. P.; Lauer-Fields, J. L.; Schmoekel, H. G.; Metters, A. T.; Weber, F. E.; Fields, G. B.; Hubbell, J. A. Proc. Natl. Acad. Sci. U. S. A. 2003, 100, 5413-5418.

[14] Zisch, A. H.; Lutolf, M. P.; Ehrbar, M.; Raeber, G. P.; Rizzi, S. C.; Davies, N.; Schmokel, H.; Bezuidenhout, D.; Djonov, V.; Zilla, P.; Hubbell, J. A. FASEB J. 2003, 17, 2260-2285.

[15] Zustiak, S. P.; Leach, J. B. Biomacromolecules 2010, 11, 1348-1357.

[16] Pritchard, C. D.; O'Shea, T. M.; Siegwart, D. J.; Calo, E.; Anderson, D. G.; Reynolds, F. M.; Thomas, J. A.; Slotkin, J. R.; Woodard, E. J.; Langer, R. Biomaterials 2011, 32, $587-$ 597.

[17] Elbert, D. L.; Hubbell, J. A. Biomacromolecules 2001, 2, 430-441.

[18] Sawhney, A. S.; Pathak, C. P.; Hubbell, J. A. Macromolecules 1993, 26, 581-587.

[19] Goessl, A.; Tirelli, N.; Hubbell, J. A. J. Biomater. Sci., Polym. Ed. 2004, 15, 895-904.

[20] Wallace, O. B.; Springer, D. M. Tetrahedron Lett. 1998, 39, 2693-2694.

[21] Ellman, G. L. Arch. Biochem. Biophys. 1959, 82, 70-77. 
[22] Peppas, N. A.; Barr-Howell, B. D. Characterization of the cross-linked structure of hydrogels. 1986, Boca Raton: CRC Press.

[23] Canal, T.; Peppas, N. A. J. Biomed. Mater. Res. 1989, 23, 1183-1193.

[24] Brandrup, J.; Immergut, E. H. Polymer Handbook. 1975, New York: Wiley.

[25] Ranbhor, R.; Ramakrishnan, V.; Kumar, A.; Durani, S. Biopolymers 2006, 83, 537-545.

[26] Buwalda, S. J.; Dijkstra, P. J.; Feijen, J. Chapter 4, this thesis; University of Twente, 2011.

[27] Buwalda, S. J.; Dijkstra, P. J.; Calucci, L.; Forte, C.; Feijen, J. Biomacromolecules 2010, 11, 224-232.

[28] Lemmouchi, Y.; Perry, M. C.; Amass, A. J.; Chakraborty, K.; Schacht, E. J. Polym. Sci., Part A: Polym. Chem. 2007, 45, 3975-3985.

[29] Metters, A.; Hubbell, J. Biomacromolecules 2005, 6, 290-301.

[30] Shikanov, A.; Smith, R. M.; Xu, M.; Woodruff, T. K.; Shea, L. D. Biomaterials 2011, 32, 2524-2531.

[31] Tung, C. Y. M.; Dynes, P. J. J. App. Polym. Sci. 1982, 27, 569-574.

[32] Yu, H. Q.; Cong, R. Chem. Pap. 2010, 64, 619-624.

[33] Schoenmakers, R. G.; van de Wetering, P.; Elbert, D. L.; Hubbell, J. A. J. Controlled Release 2004, 95, 291-300.

[34] Hiemstra, C.; Zhong, Z. Y.; van Steenbergen, M. J.; Hennink, W. E.; Feijen, J. J. Controlled Release 2007, 122, 71-78. 


\title{
In situ forming stereocomplexed and photocrosslinked PEG-PLA hydrogels. Mechanical properties, degradation and drug release
}

Parts of this chapter have been published: Sytze J. Buwalda, ${ }^{a}$ Pieter J. Dijkstra, ${ }^{a}$ and Jan Feijen ${ }^{a}$ Journal of Controlled Release 2010, 148, e23-e24.

a Department of Polymer Chemistry and Biomaterials, Faculty of Science and Technology, MIRA Institute for Biomedical Technology and Technical Medicine, University of Twente, P.O. Box 217, 7500 AE Enschede, The Netherlands

\begin{abstract}
Biodegradable poly(ethylene glycol)-poly(lactide) (PEG-PLA) hydrogels were formed by physical gelation through stereocomplexation of star shaped PEG-PLLA and PEG-PDLA enantiomers followed by UV photopolymerization of PLA terminal acrylate groups. The 8-armed PEG-PLA star block copolymers had a PEG core with a molecular weight of $20 \mathrm{~kg} / \mathrm{mol}$ and PLA blocks of 12 lactyl units connected to the PEG core via amide bonds. The PLA blocks were terminated with an acrylate end group. Aqueous solutions of enantiomeric mixtures of the PEGPLA macromonomers formed physically crosslinked hydrogels above a critical gel concentration of $4 \mathrm{w} / \mathrm{v} \%$. Subsequent photopolymerization in the presence of Irgacure 2959 resulted in gels with significantly improved mechanical properties and hydrolytic stability. The release properties of the photopolymerized gels were evaluated using lysozyme, albumin and rhodamine B as model compounds. A first order release of albumin and lysozyme over a period of $10 \mathrm{~d}$ was obtained. The release of rhodamine B from the stereocomplexed and photocrosslinked PEG-PLA hydrogels was characterized by an initial burst followed by a degradation dependent release during several months. Photocrosslinked hydrogels prepared from 8-armed star polymers with 2 hydroxyl terminated PEGPLLA arms and 6 acrylate end functionalized PEG arms were used as a reference material in the rhodamine $B$ release studies. The very slow release from these materials suggested that ion-dipole interactions of rhodamine B with the acrylamide groups may play an important role. Their excellent mechanical properties, their potential to be formed in situ and their high hydrolytic stability make these photocrosslinked PEG-PLA hydrogels interesting materials for biomedical applications and as controlled drug delivery systems.
\end{abstract}




\subsection{Introduction}

Hydrogels are highly water swollen polymer networks whose properties resemble those of natural soft tissues. ${ }^{1-3}$ Biodegradable poly(ethylene glycol)-poly(lactide) (PEG-PLA) type hydrogels generally exhibit excellent biocompatibility and are accordingly widely investigated for their use in biomedical applications such as tissue engineering and systems for controlled delivery of biologically active agents. Thermo-sensitive amphiphilic block copolymers form hydrogels through physical crosslinking. Depending on their molecular architecture and molecular weight they provide a sol to gel transition upon a decrease or increase in temperature. Such systems offer the advantages of a simple injection method when the sol to gel transition is close to body temperature. In this way surgical procedures may be omitted, the shape can be properly adapted and cells or proteins can be easily incorporated. ${ }^{4-6}$ Physical crosslinks, such as hydrophobic or ionic interactions, can be formed under mild conditions, but the resulting hydrogels generally are rapidly degraded and/or dissolved. Alternatively, chemically crosslinked hydrogels have been prepared from various combinations of macromonomers endcapped with reactive groups. Well-known examples are hydrogels formed by Michael addition between thiols and vinylic groups $\mathrm{s}^{7,8}$ or by reaction between activated esters and amines. $^{9,10}$ Most often, however, chemically crosslinked hydrogels are prepared by photocrosslinking of (meth)acrylate endcapped block copolymers with the aid of UV or visible light. Pioneering work was conducted by the group of Hubbell, who prepared photocrosslinked hydrogels from end acrylated PLA-PEG-PLA and PLGA-PEG-PLGA triblock copolymers. ${ }^{11}$ The degradation times of the hydrogels could be tuned from 1 to $120 \mathrm{~d}$ by altering the molecular weight of the PEG and the length and composition of the hydrophobic polyester block. West et al. reported on a photocrosslinked hydrogel based on PEG diacrylate that was rendered biodegradable by incorporation of a collagenase sensitive peptide sequence in the network. ${ }^{12}$ Fibroblasts were encapsulated successfully in the hydrogel by exposing a cell containing macromonomer solution briefly to UV light. An acrylated RGD (arginine-glycine-aspartic acid) cell adhesive peptide was incorporated by light-induced reaction with remaining acrylate groups in a partially crosslinked PEG diacrylate network. By using a photolithographic technique, the precise location of RGD could be dictated and cells exhibited guided three-dimensional migration only into the RGD-patterned regions of the hydrogels. The group of Anseth prepared various photocrosslinked hydrogels based on (meth)acrylate terminated PEG copolymers. It was demonstrated that the macroscopic properties and degradation of hydrogels prepared from PLA-PEG-PLA triblock copolymers endcapped with acrylate moieties could be tuned by altering the polymerization conditions. ${ }^{13}$ Furthermore, they showed that a hydrogel composed of a mixture of degradable and non-degradable PEG diacrylate macromonomers can serve as a scaffold for the engineering of cartilage. ${ }^{14}$ After implantation of the construct in mice, encapsulated chondrocytes were capable of survival and proliferation and integrated with surrounding native cartilage over time. Photocrosslinked hydrogels have also been 
used for the controlled release of various hydrophobic and hydrophilic drugs, including hydrogels based on methacrylate terminated $\mathrm{PEG}^{15}$ and PEG-poly( $\varepsilon$-caprolactone) multiblock copolymers. ${ }^{16}$ In both systems, the drug release could be regulated via the composition of the hydrogel.

It is important that during the gelation process no flow of macromonomers or collapse of the gel takes place. ${ }^{17}$ This issue has been addressed by a few research groups by combining physical and chemical crosslinking. For example, thermally induced physical crosslinking of an acrylated Pluronic was used to provide fast gelation. ${ }^{18}$ The gel was then photocrosslinked to give a highly stable gel. More recently, the group of Hennink reported on thermo-sensitive and photocrosslinked hydrogels based on biodegradable triblock copolymers of PEG and methacrylated poly(N-(2hydroxypropyl)methacrylamide lactate). ${ }^{19}$ It was found that a higher degree of methacrylation and a lower PEG molecular weight resulted in a higher storage modulus and crosslink density, which tailored the degradation from 25 to more than $300 \mathrm{~d} \cdot{ }^{20}$ It was demonstrated that the release rate of model proteins depended on the protein size and the molecular weight between hydrogel crosslinks and that the release was governed by Fickian diffusion. ${ }^{21}$

Previously we found that in situ forming, robust hydrogels can be prepared by combining stereocomplexation and photocrosslinking of methacrylate terminated enantiomeric PEG-PDLA and PEG-PLLA star block copolymers. ${ }^{22}$ Stereocomplexation provided fast gelation at relatively low concentrations while photopolymerization of the methacrylate groups yields significantly increased mechanical properties and prolonged degradation times. Recently we showed that the mechanical stability and most importantly the degradation of these star block copolymers can be controlled by replacing the highly hydrolytically labile linking ester bond with an amide bond. ${ }^{23,24}$ Based on these data, stereocomplexed and photocrosslinked hydrogels prepared from acrylate terminated PEG-PLLA and PEG-PDLA star block copolymers with an amide linkage between PEG and PLA were expected to have excellent mechanical properties and may be well applicable as an injectable, yet robust controlled drug delivery system. In this paper, the physical, mechanical and degradation properties of these stereocomplexed and photocrosslinked PEG-PLA hydrogels are described together with the release of lysozyme and albumin and the model drug rhodamine B from these systems.

\subsection{Experimental section}

Materials. Hydroxyl terminated 8-armed poly(ethylene glycol) (PEG-(OH) $8, M_{n}$, NMR $=21400$ $\mathrm{g} / \mathrm{mol}$ ) was purchased from Jenkem (Allen, Texas, USA) and purified before use by dissolution in dichloromethane and precipitation in cold diethyl ether. PEG-( $\left.\mathrm{NH}_{2}\right)_{8}$ and all block copolymers were synthesized as described previously. ${ }^{23,25}$ L-lactide and D-lactide were obtained from Purac (Gorinchem, the Netherlands). Acryloyl chloride, methanesulfonyl chloride (mesyl chloride), tin(II) 2-ethylhexanoate (stannous octoate), triethylamine (TEA), lysozyme, albumin and $25 \%$ aqueous 
ammonia solution were from Sigma-Aldrich (St Louis, Missouri, USA). Irgacure 2959 was obtained from Ciba (Basel, Switzerland). Rhodamine B was purchased from Merck (Darmstadt, Germany). Toluene, diethyl ether, methanol and dichloromethane were all from Biosolve (Valkenswaard, the Netherlands). Dichloromethane, TEA and toluene were dried over calcium hydride, potassium hydroxide and sodium, respectively, and distilled prior to use.

Synthesis. Photocrosslinked PEG-PLA hydrogels were prepared by UV irradiation of aqueous solutions of acrylate terminated PEG-PLA star block copolymers. Typically, distilled water containing $10 \mathrm{~mol} \%$ photoinitiator (Irgacure 2959) relative to acrylate groups was added to a mixture of PEG-(PDLA 12$)_{8}-\mathrm{AC}$ and PEG-(PLLA 12$)_{8}-\mathrm{AC}(\mathrm{D} / \mathrm{L}$ ratio $1 / 1)$. The system was heated repeatedly and subsequently equilibrated for $48 \mathrm{~h}$ at room temperature. The mixture was finally irradiated by UV light $\left(\sim 5 \mathrm{~mW} / \mathrm{cm}^{2}\right)$ at $365 \mathrm{~nm}$ for $30 \mathrm{~min}$ in an inert atmosphere.

Characterization. ${ }^{1} \mathrm{H}$ NMR $(300 \mathrm{MHz})$ spectra were recorded on a Varian Inova $300 \mathrm{NMR}$ spectrometer. Polymers were dissolved in $\mathrm{CDCl}_{3}$ at a concentration of $15 \mathrm{mg} / \mathrm{ml}$.

Thermal properties of polymers were determined using differential scanning calorimetry (DSC). Heating and cooling rates of $20^{\circ} \mathrm{C} / \mathrm{min}$ were applied. Samples were heated from 25 to $200{ }^{\circ} \mathrm{C}$, kept at $200{ }^{\circ} \mathrm{C}$ for $1 \mathrm{~min}$, cooled to $-50{ }^{\circ} \mathrm{C}$, kept at $-50{ }^{\circ} \mathrm{C}$ for $1 \mathrm{~min}$, and finally heated to $200{ }^{\circ} \mathrm{C}$. Crystallization temperatures $\left(\mathrm{T}_{\mathrm{c}}\right)$ and corresponding enthalpies $\left(\Delta \mathrm{H}_{\mathrm{c}}\right)$ were obtained from the cooling scan, while melting temperatures $\left(\mathrm{T}_{\mathrm{m}}\right)$ and enthalpies $\left(\Delta \mathrm{H}_{\mathrm{m}}\right)$ were obtained from the second heating scan.

Gel properties. Oscillatory rheology experiments were performed to determine the mechanical properties of the photocrosslinked hydrogels. The storage (G') and loss (G') modulus of hydrogels were monitored for $30 \mathrm{~min}$ at $25{ }^{\circ} \mathrm{C}$ on an Anton-Paar Physica MCR 301 rheometer. Experiments were performed using a flat plate measuring geometry (diameter $25 \mathrm{~mm}$, gap $0.3 \mathrm{~mm}$ ) utilizing a strain of $1 \%$ and a frequency of $1 \mathrm{~Hz}$. To prevent water evaporation, a solvent trap was placed over the geometry.

Gravimetric degradation/dissolution experiments were performed to determine the stability of the hydrogels. Freshly prepared semi-spherical hydrogel samples (radius $5 \mathrm{~mm}$ ) were dried in air, their initial weight $\mathrm{W}_{0}$ was determined and the samples were immersed in PBS at $37{ }^{\circ} \mathrm{C}$. To prevent bacterial growth, $0.02 \mathrm{w} / \mathrm{v} \% \mathrm{NaN}_{3}$ was added to the buffer solution. At regular times, samples were taken out and their mass in swollen state $\left(\mathrm{W}_{\mathrm{s}}\right)$ was measured after wiping the surface with tissue paper. Subsequently, the samples were allowed to dry in air overnight to yield the dry weight $\left(\mathrm{W}_{\mathrm{D}}\right)$. The degree of swelling during degradation was calculated from: 


$$
\text { degree of swelling }=\frac{\left(\mathrm{W}_{\mathrm{S}}-\mathrm{W}_{\mathrm{D}}\right)}{\mathrm{W}_{\mathrm{D}}} \cdot 100 \%
$$

The remaining relative polymer mass during degradation was calculated from:

$$
\text { relative polymer mass }=\frac{\mathrm{W}_{\mathrm{D}}}{\mathrm{W}_{0}} \cdot 100 \%
$$

Release experiments were performed with lysozyme and albumin as model proteins and rhodamine $\mathrm{B}$ as a model drug. The proteins were dissolved in a minimal amount of PBS and this solution was added to an $8 \mathrm{w} / \mathrm{v} \%$ stereocomplexed PEG-(PDLA 12$)_{8}$-AC / PEG-(PLLA 12$)_{8}$-AC hydrogel prepared in PBS as described above. The protein concentration was $10 \mathrm{wt} \%$ relative to the total polymer mass. After vigorous mixing, the sample was allowed to equilibrate for $24 \mathrm{~h}$. The mixture was subsequently irradiated by UV light $\left(\sim 5 \mathrm{~mW} / \mathrm{cm}^{2}\right)$ at $365 \mathrm{~nm}$ for $30 \mathrm{~min}$ in an inert atmosphere. For the in vitro release of the model proteins, the hydrogels $(0.4 \mathrm{ml})$ were placed in 5 $\mathrm{ml}$ of PBS and kept at $37^{\circ} \mathrm{C}$. At regular time intervals, $0.5 \mathrm{ml}$ of supernatant was removed and replaced by fresh buffer. The concentration of lysozyme and albumin in the release samples as well as the enzymatic activity of the lysozyme in selected release samples were determined as described previously. $^{25}$

Rhodamine B was dissolved in water $(0.1 \mathrm{mg} / \mathrm{ml})$ together with a 1:1 PEG-(PDLA 12$)_{8}$-AC / PEG$\left(\mathrm{PLLA}_{12}\right)_{8}$-AC mixture $(8$ or $16 \mathrm{w} / \mathrm{v} \%)$ or PEG-(AC) $6 /\left(\mathrm{PLLA}_{43}\right)_{2}(16 \mathrm{w} / \mathrm{v} \%)$ and $10 \mathrm{~mol} \%$ photoinitiator relative to acrylate groups. The system was heated repeatedly and subsequently equilibrated for $48 \mathrm{~h}$. The mixture was finally irradiated by UV light $\left(\sim 5 \mathrm{~mW} / \mathrm{cm}^{2}\right)$ at $365 \mathrm{~nm}$ for $150 \mathrm{~min}$ in an inert atmosphere. For the in vitro release of the model drug, hydrogel samples $(0.25$ $\mathrm{ml}$ ) were immersed in $3 \mathrm{ml}$ of PBS and kept at $37^{\circ} \mathrm{C}$. At regular time intervals, $0.2 \mathrm{ml}$ of release medium was removed and replaced by fresh buffer. The concentration of rhodamine B in the release samples was determined by reading the absorbance at $555 \mathrm{~nm}$ using a Tecan Safire microplate reader. A calibration curve was constructed using rhodamine B solutions of known concentration.

\subsection{Results and discussion}

Synthesis and characterization of acrylate terminated macromonomers. Stereocomplexation of enantiomeric PLA blocks in PEG-PLA amphiphilic block copolymers is a powerful tool to prepare physically crosslinked hydrogels. ${ }^{24}$ Although such hydrogels have good mechanical stability their degradation/dissolution rate is generally fast in physiological media. One way to overcome the fast dissolution is post crosslinking of stereocomplexed hydrogels through e.g. photocrosslinking of acrylate end functionalized block copolymers. ${ }^{22}$ The degradation properties can be tailored by varying the PLA block length and by replacing hydrolytically instable bonds, especially the ester linking unit between PLA and PEG blocks, by a hydrolytically stable amide 
bond. ${ }^{23}$ The degradation of such networks consequently is governed solely by the hydrolysis of ester bonds in the PLA chains. In previous research it was shown that highly stable PEG-PLA type networks were obtained when a methacrylate group is directly attached to the PEG blocks and subsequently photopolymerized. ${ }^{22}$ Because of its slow degradation in aqueous media the PEG$(\mathrm{AC})_{6} /\left(\mathrm{PLLA}_{43}\right)_{2}$ (Figure 1) described here was used as a reference material in release studies of a model drug from degradable and photocrosslinked 8-armed PEG-PLA copolymers.

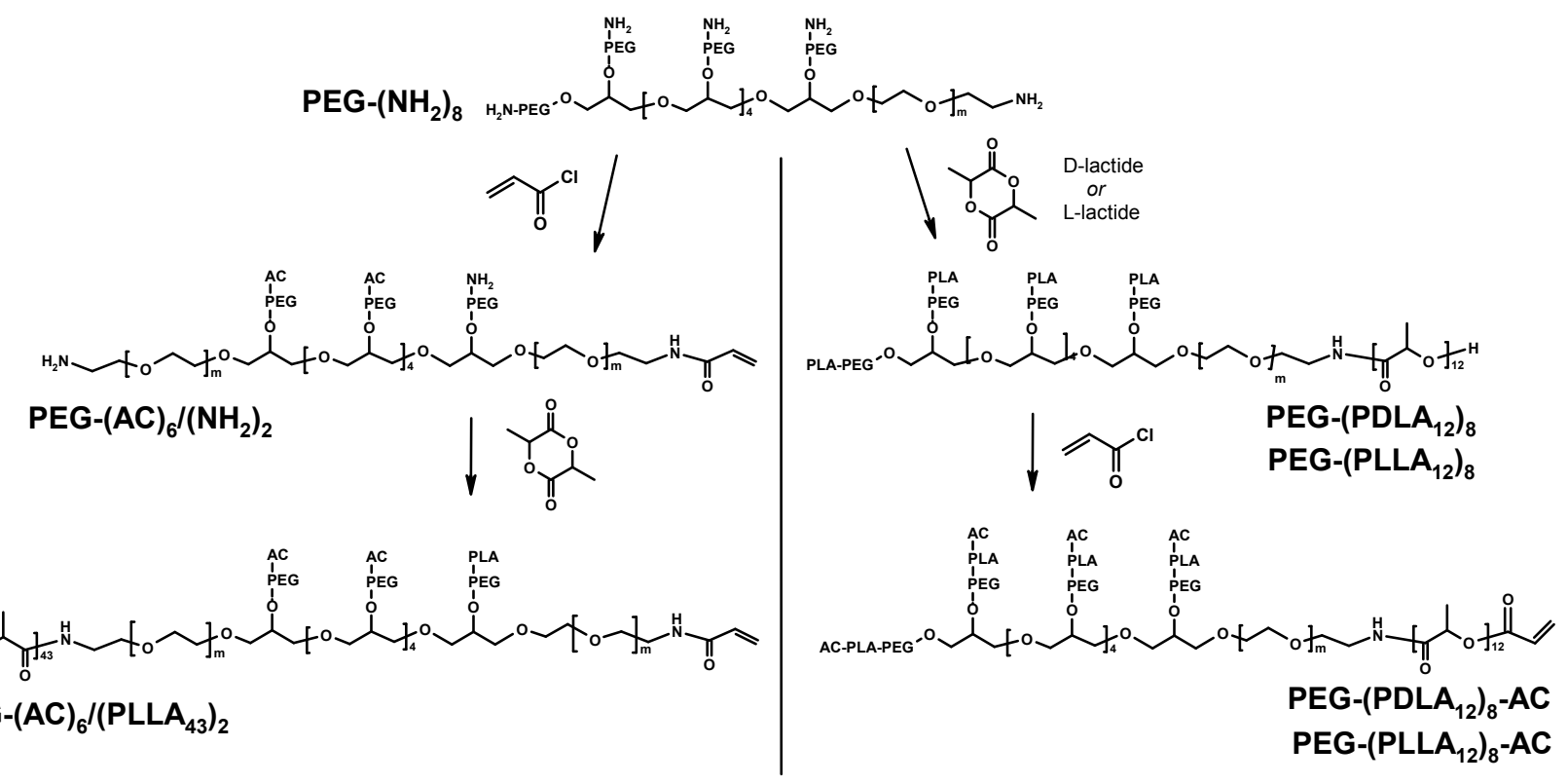

Figure 1. Synthesis scheme for the preparation of PEG-(PLA 12$)_{8}-\mathrm{AC}$ and $\mathrm{PEG}-(\mathrm{AC})_{6} /\left(\mathrm{PLLA}_{43}\right)_{2}$ star block copolymers.

PEG-(PDLA 12$)_{8}$ and PEG-(PLLA 12$)_{8}$ star block copolymers with an amide linkage between the PEG and PLA blocks were prepared by the stannous octoate catalyzed ring opening polymerization of D-lactide and L-lactide, respectively, initiated by PEG-( $\left.\mathrm{NH}_{2}\right)_{8}$ (Figure 1). Star block copolymers with a degree of polymerization (DP) of 12 lactyl units per PLA arm were synthesized (Table 1), because higher DPs led to a reduced solubility in water whereas lower DPs have been shown to hamper stereocomplex formation. ${ }^{26}$ The PEG-(PDLA 12$)_{8}$ and PEG-(PLLA 12$)_{8}$ star block copolymers were functionalized with acrylate groups by reaction with acryloyl chloride in dichloromethane as reported previously. ${ }^{25}$ The signal at $4.35 \mathrm{ppm}$ corresponding to the terminal PLA methine protons of the starting PEG-(PLA 12$)_{8}$ completely disappeared, indicating quantitative end group conversion. 
Table 1. Molecular weights, thermal properties and critical gel concentrations (CGCs) of PEG-PLA star block copolymers.

\begin{tabular}{|c|c|c|c|c|c|c|c|c|}
\hline \multirow[b]{2}{*}{ Polymer } & \multicolumn{3}{|c|}{${ }^{3}$ H NMR } & \multirow[b]{2}{*}{$\begin{array}{l}\mathrm{T}_{\mathrm{m}} \\
\left({ }^{\circ} \mathrm{C}\right)\end{array}$} & \multirow[b]{2}{*}{$\begin{array}{l}\Delta \mathrm{H}_{\mathrm{m}} \\
(\mathrm{J} / \mathrm{g})\end{array}$} & \multirow[b]{2}{*}{$\begin{array}{l}\mathrm{T}_{\mathrm{c}} \\
\left({ }^{\circ} \mathrm{C}\right)\end{array}$} & \multirow[b]{2}{*}{$\begin{array}{l}\Delta \mathrm{H}_{\mathrm{c}} \\
(\mathrm{J} / \mathrm{g})\end{array}$} & \multirow[b]{2}{*}{$\begin{array}{c}\text { CGC } \\
(\mathrm{w} / \mathrm{v} \%)\end{array}$} \\
\hline & $\begin{array}{c}\mathrm{M}_{\mathrm{n}} \\
(\mathrm{kg} / \mathrm{mol})\end{array}$ & $\mathrm{DP}^{a}$ & $\begin{array}{c}\text { Degree of } \\
\text { functionalization } \\
\text { (\% acrylate end } \\
\text { groups) }\end{array}$ & & & & & \\
\hline PEG-(NH$)_{8}$ & 21.4 & & & 53 & 126 & 28 & 126 & \\
\hline $\begin{array}{c}\text { PEG-(PDLA } 12)_{8} \\
+ \\
\text { PEG-(PLLA } 12)_{8}\end{array}$ & $\begin{array}{l}27.7 \\
28.3\end{array}$ & $\begin{array}{l}10.9 \\
11.9\end{array}$ & & 41 & 56 & 13 & 57 & 5 \\
\hline $\begin{array}{c}\left.\text { PEG-(PDLA }{ }_{12}\right)_{8} \text {-AC } \\
+ \\
\text { PEG-(PLLA } 12)_{8} \text {-AC }\end{array}$ & 28.1 & 10.9 & $\begin{array}{l}>95 \\
>95\end{array}$ & 39 & 44 & 21 & 50 & 4 \\
\hline PEG-(AC) $6 /\left(\mathrm{NH}_{2}\right)_{2}$ & 21.7 & & 75 & 47 & 86 & 27 & 84 & \\
\hline PEG-(AC) $)_{6} /\left(\text { PLLA }_{43}\right)_{2}$ & 27.9 & 43 & 75 & 46 & 59 & 25 & 58 & 12 \\
\hline
\end{tabular}

${ }^{a}$ Degree of polymerization of the PLA blocks, expressed in lactyl units per arm.

For PEG-(AC) 6 (PLLA 43$)_{2}$ first approximately $75 \%$ of the amine end groups in PEG-(NH $)_{8}$ were converted to acrylate end groups by reaction with acryloyl chloride (Figure 1). ${ }^{25}$ Subsequently, the stannous octoate catalyzed ring opening polymerization of L-lactide initiated by the remaining amine groups on the partially acrylated PEG yielded the PEG-(AC) $6 /\left(\text { PLLA }_{43}\right)_{2}$ star block copolymer. The structural differences between PEG-(PLA 12$)_{8}-\mathrm{AC}$ and PEG-(AC) 6 (PLLA 43$)_{2}$ are reflected in their ${ }^{1} \mathrm{H}$ NMR spectra (Figure 2). Compared to PEG-(PLA 12$)_{8}$-AC, additional peaks are visible in the spectrum of PEG-(AC) 6 ( PLLA $\left._{43}\right)_{2}$ corresponding to the acrylamide protons at 6.8 ppm and the terminal PLA methine protons at $4.35 \mathrm{ppm}$. Furthermore signals corresponding to the vinylic protons appear more upfield resulting from the conjugation with the amide group. 

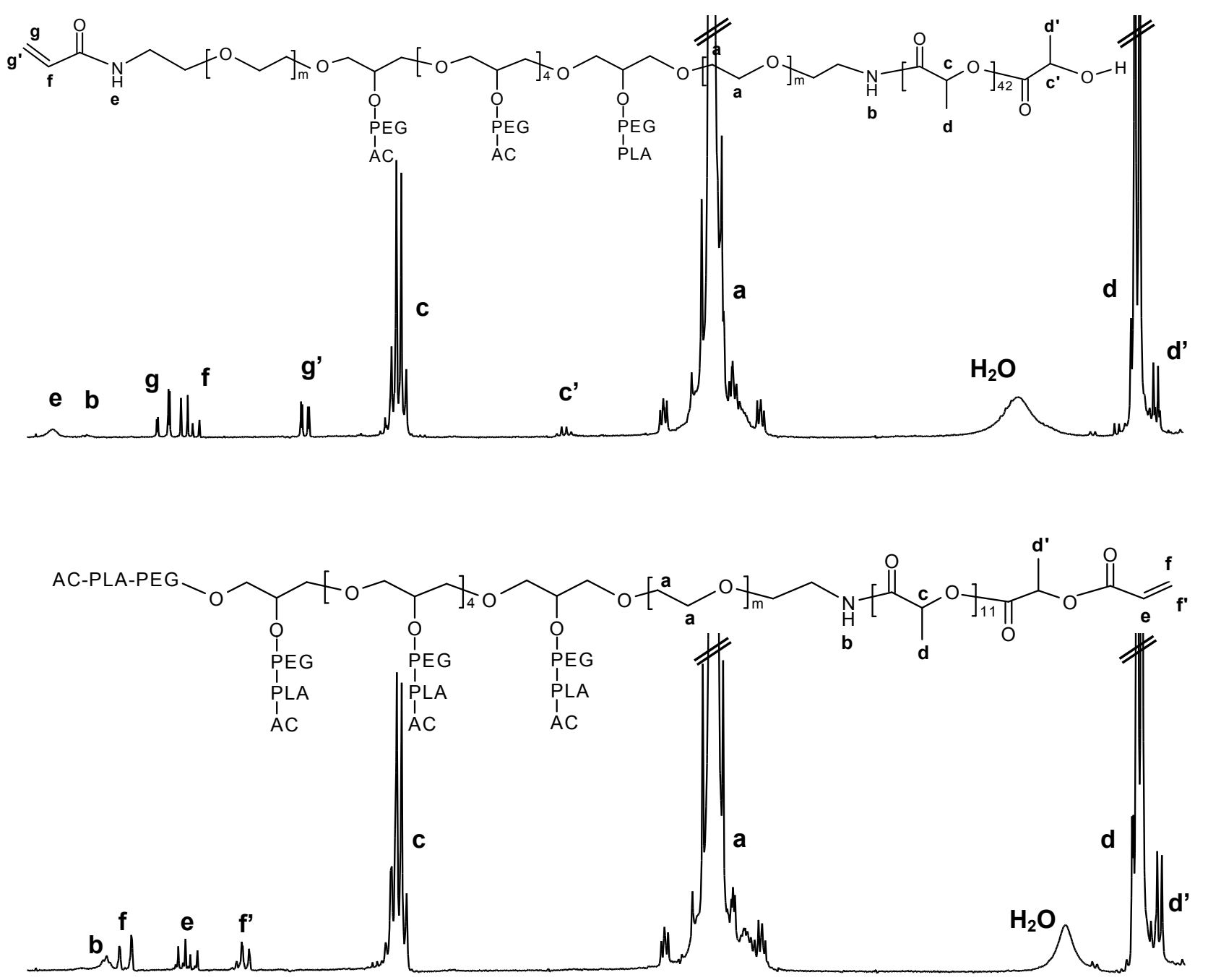

$\begin{array}{lllllllllllllllllllllllllllllllll}6.8 & 6.6 & 6.4 & 6.2 & 6.0 & 5.8 & 5.6 & 5.4 & 5.2 & 5.0 & 4.8 & 4.6 & 4.4 & 4.2 & 4.0 & 3.8 & 3.6 & 3.4 & 3.2 & 3.0 & 2.8 & 2.6 & 2.4 & 2.2 & 2.0 & 1.8 & 1.6 & 1.4\end{array}$

Figure 2. ${ }^{1} \mathrm{H}$ NMR spectra of PEG-(PLLA 12$)_{8}-\mathrm{AC}$ (bottom) and PEG-(AC)6 6 (PLLA 43$)_{2}$ (top). Solvent: $\mathrm{CDCl}_{3}$.

The thermal properties of enantiomeric mixtures of the block copolymers as determined with differential scanning calorimetry (DSC) revealed major transitions in both second heating and cooling scans, corresponding to melting and crystallization of the PEG domains, respectively (Table 1). The PEG-PLA star block copolymers exhibit lower melting transitions and accompanying enthalpies than their PEG precursors indicating that the crystallization of PEG is hampered by the presence of the PLA blocks. Functionalization of PEG-PLA with acrylate groups further impedes PEG crystallization as shown by the lower $\mathrm{T}_{\mathrm{m}}$ and $\Delta \mathrm{H}_{\mathrm{m}}$ values of the enantiomeric PEG-(PLA $\left.{ }_{12}\right)_{8^{-}}$ AC mixture. A melting transition of the PLA phase in the single enantiomer block copolymers was not observed. Occasionally, for the stereocomplexed PLA phase we could observe a melting transition at $\sim 160{ }^{\circ} \mathrm{C}$ with a low $\Delta \mathrm{H}_{\mathrm{m}}$ value depending on the heating and cooling rates. ${ }^{24} \mathrm{~A}$ small 
exothermic peak was observed at $140{ }^{\circ} \mathrm{C}$ for PEG-(AC) 6 ( PLLA $\left._{43}\right)_{2}$, corresponding to melting of PLLA domains formed by the relatively long PLLA arms.

Self-assembly and physical gelation of PEG-PLA star block copolymers in water. PEG-PLA type block copolymers typically self-assemble in water to form micelles and small aggregates with a hydrophobic PLA core and a hydrophilic PEG corona at concentrations above the critical association concentration (CAC). ${ }^{1} \mathrm{H}$ NMR analysis revealed that also PLA domains and free dangling PLA chains are present in the periphery of the aggregates. ${ }^{27}$ The CAC values depend on several parameters including the PEG/PLA molecular weight ratio and the possibility to form stereocomplexes between PDLA and PLLA blocks. The CACs of mixed enantiomers of PEG$\left(\mathrm{PLA}_{\mathrm{n}}\right)_{8}$ are approximately 2 to 3 times lower than the values of the corresponding single enantiomers $(\sim 0.4 \mathrm{w} / \mathrm{v} \%)$ due to the stronger interactions in the PEG-PLA stereocomplexes. ${ }^{23,24}$

Using dynamic light scattering (DLS) the aggregate sizes and aggregate size distributions of PEG-PLA star block copolymers in water were measured at a concentration of $0.5 \mathrm{w} / \mathrm{v} \%$ (Figure 3).

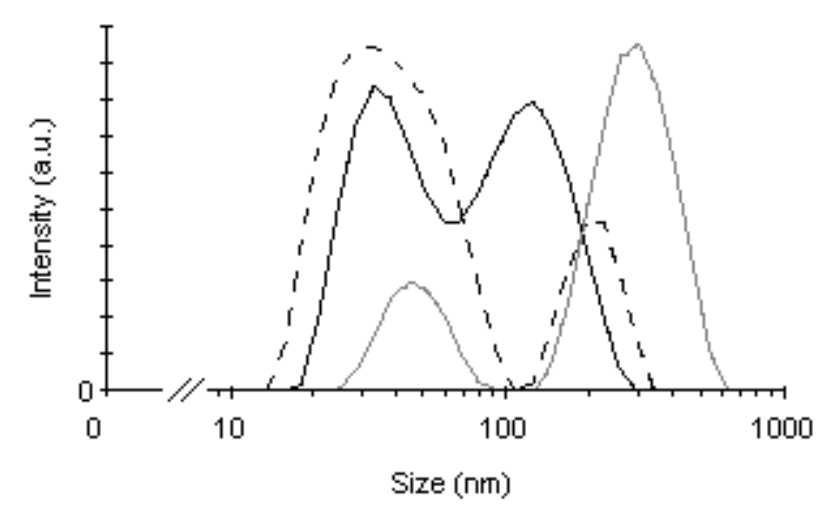

Figure 3. Aggregate size distributions in $0.5 \mathrm{w} / \mathrm{v} \%$ aqueous solutions at room temperature. PEG$\left(\text { PLLA }_{12}\right)_{8}$ (dashed line), PEG-(PDLA 12$)_{8} /$ PEG-(PLLA 12$)_{8}$ (grey line), PEG-(PDLA 12$)_{8}$-AC/PEG$\left(\mathrm{PLLA}_{12}\right)_{8}$-AC (black line).

Above the CAC small aggregates with diameters ranging from 40-50 nm, indicative of aggregated micelles, as well as larger aggregates with a diameter ranging from 100 to $300 \mathrm{~nm}$ were observed. The distributions presented in Figure 3 show that stereocomplexation between enantiomeric block copolymers results in larger aggregates. It has to be noted that the volume fractions of these larger aggregates $(0.4 \%, 17 \% \text { and } \sim 0.1 \% \text { for PEG-(PLLA } 12)_{8}$, PEG$\left(\mathrm{PDLA}_{12}\right)_{8} / \mathrm{PEG}\left(\mathrm{PLLA}_{12}\right)_{8}$ and PEG-(PDLA 12$)_{8}$-AC/PEG-(PLLA 12$)_{8}-\mathrm{AC}$, respectively) is still small compared to the small aggregates. Upon acrylation the size distribution of the larger aggregates is shifted to lower values, which may be due to the increased hydrophobicity of the 
acrylate functionalized PLA chains resulting in an enhanced aggregation tendency of these PLA arms and hence more compact larger aggregates.

The physical gelation of the 8-armed block copolymers was studied by dissolving an appropriate amount of the single enantiomer PEG-(PLLA 12$)_{8}$, stereocomplexed PEG-(PDLA 12$)_{8} /$ PEG$\left(\mathrm{PLLA}_{12}\right)_{8}$ mixture or PEG-(PDLA 12$)_{8}$-AC/PEG-(PLLA $\left.{ }_{12}\right)_{8}$-AC $(1: 1$ weight ratio) mixture in distilled water. Previously we showed by rheology experiments that the formation of stereocomplexed domains in hydrogels of such mixed enantiomers is enhanced when the temperature is elevated. ${ }^{24}$ Upon heating, the increased mobility of the PLA chains facilitates intermicellar and interaggregate bridging to a greater extent. The enhanced formation of stereocomplexes and the high stability of the stereocomplexed domains prohibit gel to sol transitions at elevated temperatures and the formation of such hydrogels is irreversible. Therefore in the current research multiple heating cycles were applied, followed by an equilibration time of $48 \mathrm{~h}$ at room temperature, to allow an optimal formation of stereocomplexes. A critical gelation concentration is observed for the PEG-(PLLA 12$)_{8}$ and PEG-(PLLA 12$)_{8}$-AC single enantiomers of 12 and $6 \mathrm{w} / \mathrm{v} \%$, respectively. At these concentrations still a thermo-reversible gel-sol transition is observed. The stereocomplexed PEG-(PDLA 12$)_{8} /$ PEG-(PLLA 12$)_{8}$ and PEG-(PDLA 12$)_{8}$-AC/PEG$\left(\mathrm{PLLA}_{12}\right)_{8}$-AC behave differently. The minimum concentrations to form a hydrogel are 5 and $4 \mathrm{w} / \mathrm{v}$ $\%$ for the PEG-(PDLA 12$)_{8} /$ PEG-(PLLA 12$)_{8}$ and PEG-(PDLA 12$)_{8}$-AC/PEG-(PLLA 12$)_{8}$-AC systems, respectively (Table 1). Above these concentrations gel formation is irreversible and below these concentrations no gels are formed. Consequently an operation window exists in which in situ hydrogel formation is facilitated by mixing aqueous solutions of single enantiomers. Oscillatory rheology measurements revealed that $7 \mathrm{w} / \mathrm{v} \%$ PEG-(PDLA 12$)_{8} / \mathrm{PEG}-\left(\mathrm{PLLA}_{12}\right)_{8}$ and $7 \mathrm{w} / \mathrm{v} \%$ PEG$\left(\mathrm{PDLA}_{12}\right)_{8}$-AC/PEG-(PLLA 12$)_{8}$-AC hydrogels exhibit storage moduli of 630 and $710 \mathrm{~Pa}$, respectively. The higher storage modulus of the PEG-(PDLA 12$)_{8}-\mathrm{AC} / \mathrm{PEG}-\left(\mathrm{PLLA}_{12}\right)_{8}$-AC hydrogel is a further indication that end group acrylation results in increased formation of the stereocomplexed domains and thus increased physical crosslinking.

The single enantiomer PEG-(AC) $)_{6} /\left(\mathrm{PLLA}_{43}\right)_{2}$ macromonomer forms physically crosslinked hydrogels at concentrations above $12 \mathrm{w} / \mathrm{v} \%$ (Table 1). Upon heating, the PEG-(AC)6/(PLLA 43$)_{2}$ hydrogels exhibit a phase transition from the gel to the sol state which is fully reversible upon cooling. At a concentration of $14 \mathrm{w} / \mathrm{v} \%$, the gel-sol transitions occur just above body temperature, which makes PEG-(AC) 6 ( $\left(\text { PLLA }_{43}\right)_{2}$ hydrogels applicable for in situ gel formation as well.

Photocrosslinked PEG-PLA hydrogels. Physically crosslinked stereocomplexed hydrogels of PEG-(PDLA 12$)_{8}$-AC and PEG-(PLLA 12$)_{8}$-AC were prepared at different concentrations in distilled water containing Irgacure 2959 as a photoinitiator. To stabilize the initially formed stereocomplexed hydrogel subsequent crosslinking by photopolymerization of the acrylate groups 
using UV irradiation was carried out. In preliminary experiments the effects of UV wavelength, reaction time and initiator concentration on the mechanical properties of the resulting hydrogels were determined by oscillatory rheology measurements. It followed that an initiator concentration of $10 \mathrm{~mol} \%$ relative to acrylate groups, an UV irradiation time of $30 \mathrm{~min}$ and an UV irradiation wavelength of $365 \mathrm{~nm}$ resulted in the highest storage modulus. An UV irradiation intensity of 5 $\mathrm{mW} / \mathrm{cm}^{2}$ was used, which is low compared to commonly used intensities for photopolymerized hydrogel systems. Although the highest storage modulus was obtained after $30 \mathrm{~min}$, a substantial increase in G' was already observed after a short irradiation time of $5 \mathrm{~min}$. Low UV intensities and short irradiation times are preferred to prevent cell damage when these systems are photopolymerized. ${ }^{28}$

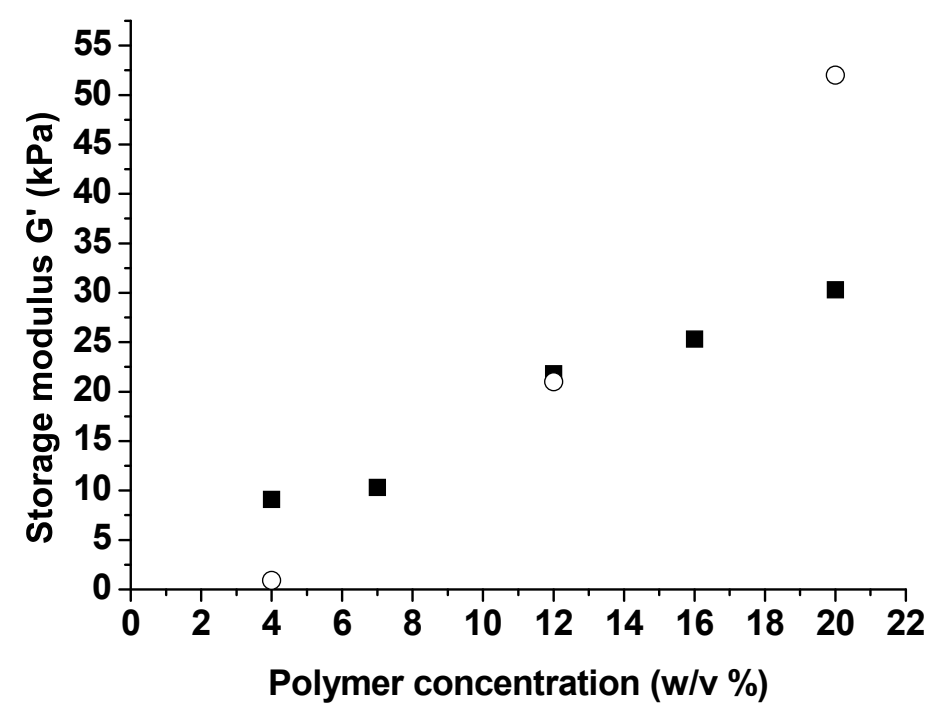

Figure 4. Storage modulus $\left(G^{\prime}\right)$ as a function of the initial polymer concentration at $25{ }^{\circ} \mathrm{C}$ for photopolymerized hydrogels based on PEG-(PDLA 12$)_{8}$-AC/PEG-(PLLA 12$)_{8}$-AC (ם) and PEG$(\mathrm{AC})_{6} /\left(\mathrm{PLLA}_{43}\right)_{2}(\mathrm{O})$.

The effect of the polymer concentration on the storage modulus was investigated using the optimized irradiation conditions (Figure 4). The increase in G' with concentration can be ascribed to the formation of a more densely crosslinked network at higher concentrations. UV irradiation of a stereocomplexed $7 \mathrm{w} / \mathrm{v} \%$ PEG-(PDLA 12$)_{8}$-AC/PEG-(PLLA 12$)_{8}$-AC hydrogel resulted in an increase of $\mathrm{G}^{\prime}$ from $0.71 \mathrm{kPa}$ to $10.3 \mathrm{kPa}$, indicating that the introduction of chemical crosslinks by polymerization of the acrylate groups to polyacrylate chains significantly improves the mechanical properties. Compared to stereocomplexed and photocrosslinked hydrogels prepared from partially methacrylated PEG-PLA star block copolymers (degree of methacrylation $40 \%$ ) with an ester linkage between PEG and PLA (PEG-(OCO)-(PLA 12$\left.)_{8}-\mathrm{METHAC}\right){ }^{22}$ these stereocomplexed and photocrosslinked PEG-(PLA 12$)_{8}$-AC hydrogels display a much higher storage modulus. As an example, a $15 \mathrm{w} / \mathrm{v} \%$ stereocomplexed and photocrosslinked PEG-(OCO)-(PLA 12$)_{8}$-METHAC 
hydrogel has a storage modulus of $9 \mathrm{kPa}$, whereas a $16 \mathrm{w} / \mathrm{v} \%$ stereocomplexed and photocrosslinked PEG-(PLA $\left.{ }_{12}\right)_{8}$-AC hydrogel has a storage modulus of $25 \mathrm{kPa}$. Moreover, the stereocomplexed and photocrosslinked PEG-(PLA 12$)_{8}$-AC hydrogels show significantly lower damping factors ( $\tan \delta=\mathrm{G}^{\prime}$ '/G') compared to stereocomplexed and photocrosslinked PEG-(OCO)$\left(\text { PLA }_{12}\right)_{8}$-METHAC hydrogels ${ }^{29}$ (data not shown). The much higher degree of acrylate end group functionalization $(>95 \%)$ in the current system results in a higher crosslink density and more elastic behavior of the stereocomplexed and photocrosslinked PEG-(PLA 12$)_{8}$-AC hydrogels. Censi et al. reported $95 \%$ conversion of vinylic groups when methacrylated PEG-PNiPAM block copolymers were photocrosslinked for $5 \mathrm{~min}$ at a macromonomer concentration of $20 \mathrm{w} / \mathrm{v} \%{ }^{21}$ The conversion was found to increase with decreasing macromonomer concentration. Since the PEG-(PDLA 12$)_{8}$ AC/PEG-(PLLA 12$)_{8}$-AC hydrogels were UV irradiated for $30 \mathrm{~min}$ at macromonomer concentrations at or below $20 \mathrm{w} / \mathrm{v} \%$, quantitative conversion of acrylate groups to polyacrylate chains is assumed.

UV irradiation of aqueous PEG-(AC) $6 /\left(\mathrm{PLLA}_{43}\right)_{2}$ solutions also leads to photocrosslinked hydrogels (Figure 4). Especially above the CGC of PEG-(AC) $6 /\left(\mathrm{PLLA}_{43}\right)_{2}(12 \mathrm{w} / \mathrm{v} \%)$ networks with a high storage modulus are obtained. These rheological experiments show that the mechanical properties of the photocrosslinked PEG-PLA hydrogels can be tuned within a broad range by altering the polymer concentration.

Hydrogel degradation. The in vitro degradation of stereocomplexed photocrosslinked PEG-PLA hydrogels was investigated by a gravimetrical procedure. The polymer mass loss, as well as the swelling of the hydrogels during degradation was monitored up to 9 weeks (Figure 5). PEG$\left(\text { PDLA }_{12}\right)_{8}$-AC/PEG-(PLLA 12$)_{8}$-AC hydrogels $(12 \mathrm{w} / \mathrm{v} \%$ in water) were prepared as described above, photocrosslinked and the gels were immersed in PBS at $37{ }^{\circ} \mathrm{C}$. At regular time intervals, hydrogels were taken out and their mass in the swollen state was determined. Subsequently, the hydrogels were allowed to dry overnight to yield the dry polymer weight after degradation. It follows from Figure 5 that the stereocomplexed and photocrosslinked PEG-(PLA 12$)_{8}$-AC hydrogels show excellent stability with approximately $40 \%$ polymer mass loss after $45 \mathrm{~d}$ in vitro. In sharp contrast, $14 \mathrm{w} / \mathrm{v} \%$ PEG-(PLA 12$)_{8}$ hydrogels crosslinked only by stereocomplexation completely dissolved after $30 \mathrm{~d}$ in vitro. ${ }^{24}$ 

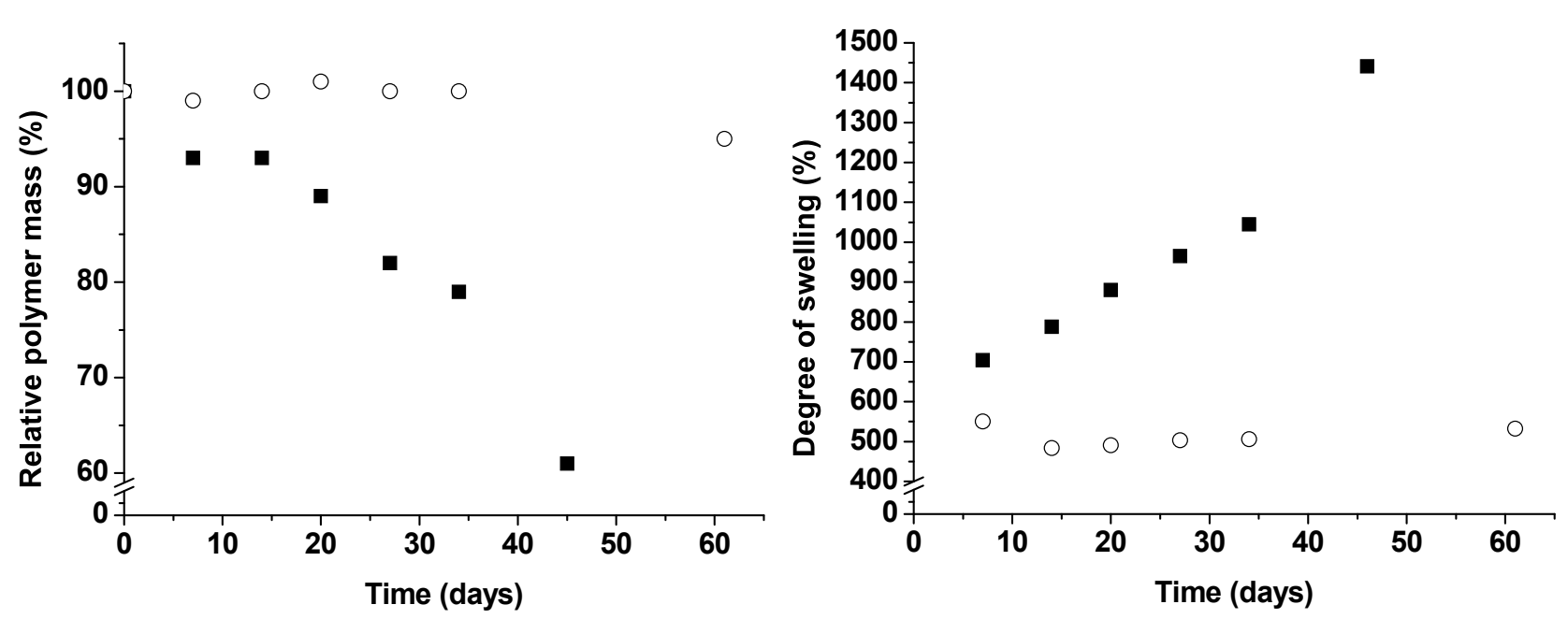

Figure 5. Polymer mass (left) and degree of swelling (right) versus time for $12 \mathrm{~W} / \mathrm{v} \%$

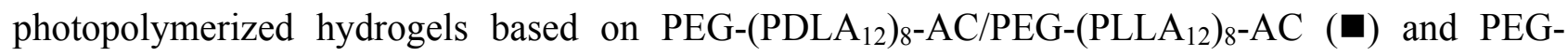
$(\mathrm{AC})_{6} /\left(\mathrm{PLLA}_{43}\right)_{2}(\mathrm{O})$ in $\mathrm{PBS}$ at $37^{\circ} \mathrm{C}$.

The degradation of the networks is accompanied by an increase in swelling (Figure 5, right). These results are likely due to the loss of physical and chemical crosslinks as a result of hydrolysis of ester bonds in the PLA blocks, which disrupts the PDLA/PLLA stereocomplex crystallites acting as physical crosslinks. Consequently lactic acid, PLA oligomers as well as poly(ethylene glycol) and poly(acrylate) macromolecules will diffuse out of the network as a result of the concentration difference between the hydrogel and the surrounding medium. Eventually, the system will dissolve completely. Oscillatory rheology measurements showed that the storage modulus of the stereocomplexed and photocrosslinked PEG-(PLA 12$)_{8}$-AC hydrogels decreased from $21.8 \mathrm{kPa}$ at the start of the degradation experiments to $0.04 \mathrm{kPa}$ after $75 \mathrm{~d}$. This was an expected behavior because $\mathrm{G}^{\prime}$ is directly related to the crosslink density. Altering the polymer concentration prior to photopolymerization did not significantly influence the relative mass loss rate, as $8 \mathrm{w} / \mathrm{v} \%$ and 20 w/v \% stereocomplexed and photocrosslinked PEG-(PLA 12$)_{8}$-AC hydrogels lost 33 and $43 \%$ of polymer mass after $45 \mathrm{~d}$, respectively. The stereocomplexed and photocrosslinked PEG-(PLA $\left.{ }_{12}\right)_{8^{-}}$ AC hydrogels are remarkably more stable than stereocomplexed and photocrosslinked PEG-(OCO)$\left(\text { PLA }_{12}\right)_{8}$-METHAC hydrogels, which completely dissolved within 4 weeks. $^{22}$ Previously we showed that the degradation of the PEG-(OCO)-(PLA) 8 block copolymer proceeds through preferential hydrolysis of the linking ester group resulting in rapid loss of PLA arms. ${ }^{23}$ Stereocomplexed PEG-(OCO)-(PLA) 8 hydrogels consequently loose their structural integrity relatively fast because the micellar packing is disrupted due to the decreased interactions between PLA arms. ${ }^{24}$ In contrast, the stable amide linking unit in stereocomplexed PEG-(PLA) 8 hydrogels only allows degradation to take place via hydrolysis of ester groups in the PLA chains, affording materials that are more slowly degrading. 
The photocrosslinked PEG-(AC) 6 /(PLLA 43$)_{2}$ networks exhibit a very slow degradation rate with 5 $\%$ mass loss after 9 weeks (Figure 5). The slight decrease in swelling during the first week of immersion may be due to the loss of non-crosslinked material. The mass loss and slight increase in swelling at later time points are most probably due to PLA degradation, upon which hydrophobic interactions acting as physical crosslinks are lost, resulting in a less densely crosslinked network. The slow mass loss confirms the idea that the acrylamide linkages between PEG and the poly(acrylate) chains are not affected and that no chemical crosslinks are lost on the time scale of the experiment. When all PLA is degraded, corresponding to $20 \mathrm{wt} \%$ of the total polymer mass, a network remains that is not or very slowly degrading. Oscillatory rheology measurements confirmed the high stability of the photocrosslinked PEG-(AC) $6 /\left(\text { PLLA }_{43}\right)_{2}$ hydrogels. The storage modulus of the photocrosslinked PEG-(AC) $6 /\left(\mathrm{PLLA}_{43}\right)_{2}$ decreased from $21.0 \mathrm{kPa}$ at day 0 to $5 \mathrm{kPa}$ at day 75 resulting from the loss of only physical crosslinks, which is a far less pronounced decrease compared to stereocomplexed and photocrosslinked PEG-(PLA 12$)_{8}$-AC hydrogels.

Release of model compounds. Injectable hydrogels are interesting materials to be used in the controlled delivery of biologically active agents. The proteins lysozyme and albumin and the dye rhodamine B were chosen as model compounds in release experiments. Lysozyme (hydrodynamic radius $4.1 \mathrm{~nm})$ is released from $8 \mathrm{w} / \mathrm{v} \%$ stereocomplexed and photocrosslinked PEG-(PLA 12$)_{8}$ hydrogels for $10 \mathrm{~d}$ (Figure 6, left), after which the release levels off. The release of albumin (hydrodynamic radius $7.6 \mathrm{~nm}$ ) follows a similar profile, but its release is slightly delayed.
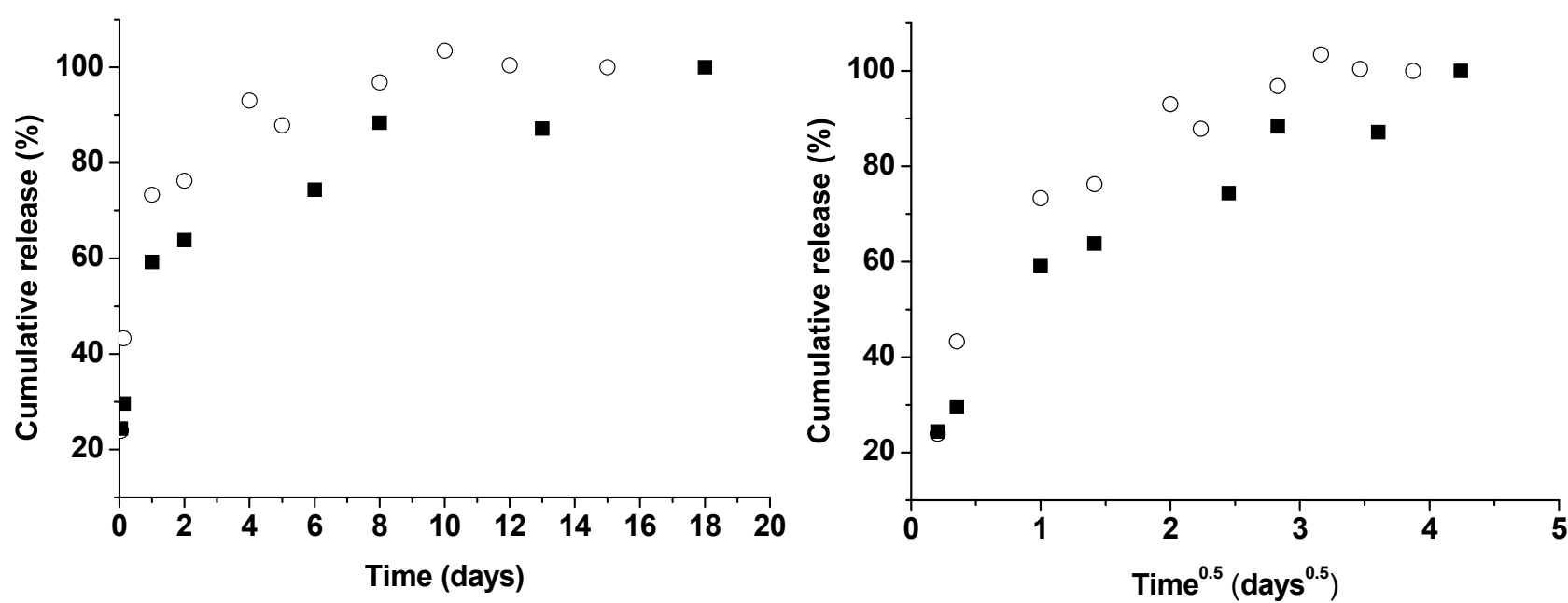

Figure 6. In vitro release of lysozyme $(\bigcirc)$ and albumin $(\mathbf{\square})$ from $8 \mathrm{w} / \mathrm{v} \%$ stereocomplexed and photocrosslinked PEG-(PLA $\left.{ }_{12}\right)_{8}$ hydrogels. Cumulative release as a function of time (left); cumulative release as a function of the square root of time (right).

Hydrogels were loaded with albumin or lysozyme by vigorously mixing a stereocomplexed PEG$\left(\mathrm{PDLA}_{12}\right)_{8}$-AC/PEG-(PLLA 12$)_{8}$-AC hydrogel with a concentrated protein solution. After 
equilibration for $24 \mathrm{~h}$, the mixture was photocrosslinked for $30 \mathrm{~min}$. The burst release during the first day may represent protein that was not distributed homogeneously throughout the hydrogel during the preparation process and that was located near the surface. After day 1, the release of lysozyme and albumin is proportional to the square root of time, indicating that the release is first order (Figure 6, right). Because during the first 3 weeks only minor degradation takes place in the stereocomplexed and photocrosslinked PEG-(PLA $\left.\mathrm{P}_{12}\right)_{8}$ hydrogels (Figure 5), these results suggest that the release of lysozyme and albumin is diffusion controlled.

Bacteria lysis experiments performed on samples from the release medium after $4 \mathrm{~d}$ showed that the released lysozyme maintained an activity of $33 \%$ relative to a control (lysozyme solution kept at $37{ }^{\circ} \mathrm{C}$ for $4 \mathrm{~d}$ ). These results may be due to denaturation of some of the lysozyme by UV irradiation and radicals generated during the photocrosslinking of the stereocomplexed hydrogel. ${ }^{30}$ The UV source used in this research emits a spectrum with a maximal intensity at $365 \mathrm{~nm}$. Especially UV light at low wavelengths is harmful for labile biological substances such as proteins. Placement of a glass filter that cuts off light below $300 \mathrm{~nm}$ between the UV light source and the hydrogel has been shown to prevent protein denaturation during in situ photocrosslinking. ${ }^{20}$

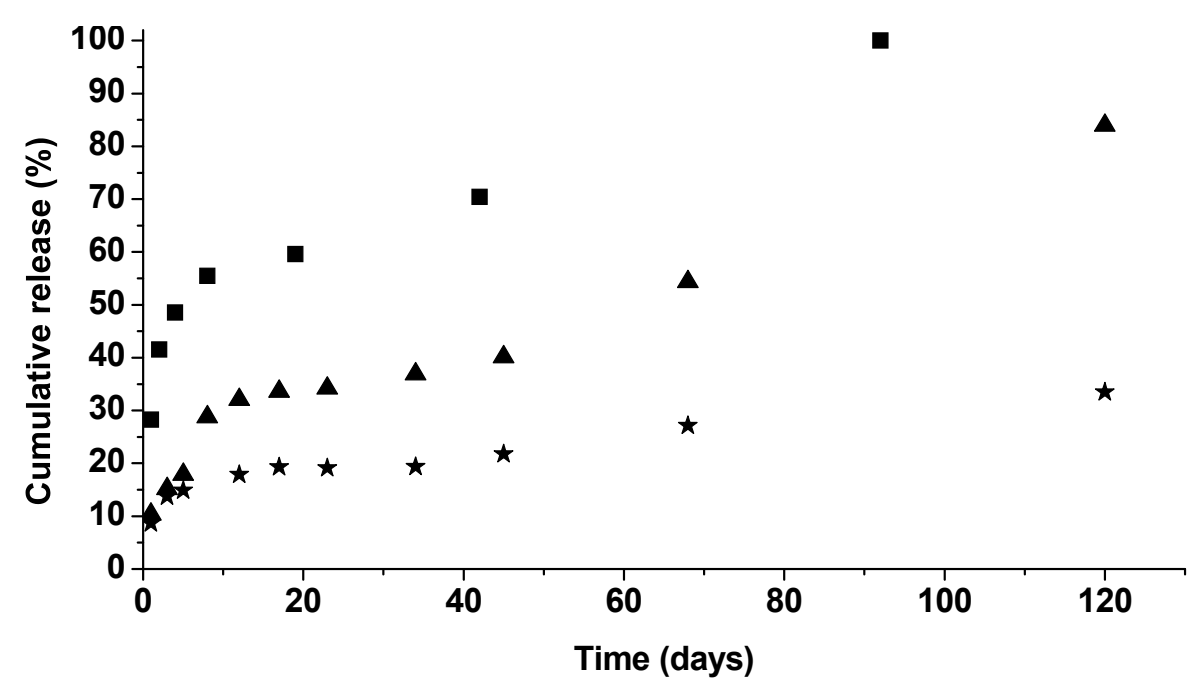

Figure 7. In vitro release of rhodamine $\mathrm{B}$ from various photocrosslinked hydrogels. Hydrogels were prepared using 8 w/v \% PEG-(PDLA 12$)_{8}$-AC/ PEG-(PLLA 12$)_{8}$-AC (ם), 16 w/v \% PEG-(PDLA 12$)_{8}$ AC/PEG-(PLLA 12$)_{8}$-AC $(\boldsymbol{\Delta})$ or 16 w/v \% PEG-(AC) $6 /\left(\operatorname{PLLA}_{43}\right)_{2}(\star)$.

The model compound rhodamine B, having a molecular weight of $480 \mathrm{~g} / \mathrm{mol}$, was loaded at a concentration of $0.1 \mathrm{mg} / \mathrm{ml}$ by dissolving the dye in the PEG-PLA macromonomer solutions prior to photocrosslinking. Figure 7 shows that $55 \%$ of the incorporated rhodamine B is released from 8 w/v \% stereocomplexed and photocrosslinked PEG-(PLA $\left.\mathrm{PL}_{12}\right)_{8}$ hydrogels during the first $8 \mathrm{~d}$. Subsequently, the release profile levels off and the cumulative release increases from 55 to $70 \%$ between day 8 and day 42 . The release from $16 \mathrm{w} / \mathrm{v} \%$ stereocomplexed and photocrosslinked PEG- 
$\left(\mathrm{PLA}_{12}\right)_{8}$ hydrogels exhibits a similar profile with a relatively fast release during the first $10 \mathrm{~d}$ which is followed by slower release kinetics. This biphasic profile may be the result of partitioning of rhodamine B in different domains of the network. Rhodamine B is a relatively hydrophilic molecule with a maximum solubility in water of $50 \mathrm{~g} / 1$ and a hydrodynamic radius of approximately $0.8 \mathrm{~nm}$. The fast initial release observed (Figure 7) most likely corresponds to rhodamine B present in the hydrophilic PEG phase. The release from this highly hydrated phase is probably diffusion controlled, given the small hydrodynamic radius of rhodamine B relative to the initial mesh size in photocrosslinked hydrogels. The mesh size is assumed to be larger than $8 \mathrm{~nm}$ based on the similar release curves of lysozyme $(4.1 \mathrm{~nm})$ and albumin $(7.6 \mathrm{~nm})$ (Figure 6).

The slower release observed after approximately $10 \mathrm{~d}$ may represent rhodamine $\mathrm{B}$ confined to the hydrophobic PLA domains. Rhodamine B is a zwitterionic molecule and has been shown to interact with polymers containing charged groups. ${ }^{31,32}$ Possibly, rhodamine B is also present in PLA rich domains as a result of ion-dipole interactions. The release from these hydrophobic domains is likely governed by degradation of the PLA and the accelerated release rate after approximately $40 \mathrm{~d}$ is probably caused by substantial degradation and consequent mass loss (Figure 5). The 8 w/v \% stereocomplexed and photocrosslinked PEG-(PLA 12$)_{8}$ hydrogel was completely degraded and dissolved after $90 \mathrm{~d}$, whereas the $16 \mathrm{w} / \mathrm{v} \%$ stereocomplexed and photocrosslinked PEG-(PLA 12$)_{8}$ hydrogel maintained its integrity and continued to release rhodamine B up to $120 \mathrm{~d}$. After an initial small burst release the release rate from photocrosslinked PEG-(AC) $6 /\left(\mathrm{PLLA}_{43}\right)_{2}$ hydrogels is very slow. This material retains its network structure even after degradation of the PLA chains. These results suggest that ion-dipole interactions with the substituted amide groups in the poly(N-PEG acrylamide) formed after photocrosslinking may play an important role. It can also not be excluded that a substantial part of the rhodamine B is entrapped in vesicular type structures present in the hydrogel. The membrane of these structures will be composed of PLLA, which is impenetrable for charged species. Further investigations are required to elucidate the exact mechanism.

\subsection{Conclusions}

PEG-PLA star block copolymer hydrogels were prepared by physical crosslinking in combination with photopolymerization. Stereocomplexation of enantiomeric PEG-PLA star block copolymers was used for facile mixing of polymers with a model drug as well as gelation in situ. Photopolymerization of acrylate end group functionalized polymers improved the mechanical properties and stability of the formed gels. The PEG-(PLA $\left.\mathrm{PL}_{12}\right)_{8}$-AC polymers could be physically crosslinked by stereocomplexation at very low concentrations and, upon photopolymerization, afforded highly robust and stable hydrogels. These PEG-(PLA $\left.{ }_{12}\right)_{8}$ gels degraded by hydrolysis of ester groups in the PLA chains resulting in a loss of physical and chemical crosslinks. Rhodamine B was released from the networks in two distinct stages as a result of partitioning of the drug in 
different domains. A relatively fast, diffusion controlled release from hydrophilic PEG domains was observed during the early stages, whereas degradation controlled release from PLA domains became important at later time points. The very slow release from the photocrosslinked PEG$(\mathrm{AC})_{6} /\left(\mathrm{PLLA}_{43}\right)_{2}$ hydrogels suggests strong ion-dipole interactions of rhodamine $\mathrm{B}$ with the acrylamide groups. This study shows that injectable, photocrosslinked PEG-PLA star block copolymer hydrogels are promising materials for applications in controlled drug delivery.

\subsection{References}

[1] Peppas, N. A.; Bures, P.; Leobandung, W.; Ichikawa, H. Eur. J. Pharm. Biopharm. 2000, 50, 27-46.

[2] Drury, J. L.; Mooney, D. J. Biomaterials 2003, 24, 4337-4351.

[3] Hennink, W. E.; van Nostrum, C. F. Adv. Drug Delivery Rev. 2002, 54, 13-36.

[4] Yu, L.; Ding, J. D. Chem. Soc. Rev. 2008, 37, 1473-1481.

[5] Van Tomme, S. R.; Storm, G.; Hennink, W. E. Int. J. Pharm. 2008, 355, 1-18.

[6] Ruel-Gariepy, E.; Leroux, J. C. Eur. J. Pharm. Biopharm. 2004, 58, 409-426.

[7] Zustiak, S. P.; Leach, J. B. Biomacromolecules 2010, 11, 1348-1357.

[8] Pritchard, C. D.; O'Shea, T. M.; Siegwart, D. J.; Calo, E.; Anderson, D. G.; Reynolds, F. M.; Thomas, J. A.; Slotkin, J. R.; Woodard, E. J.; Langer, R. Biomaterials 2011, 32, 587 597.

[9] Brandl, F.; Kastner, F.; Gschwind, R. M.; Blunk, T.; Tessmar, J.; Gopferich, A. J. Controlled Release, 2010, 142, 221-228.

[10] Kurakazu, M.; Katashima, T.; Chijiishi, M.; Nishi, K.; Akagi, Y.; Matsunaga, T.; Shibayama, M.; Chung, U.; Sakai, T. Macromolecules 2010, 43, 3935-3940.

[11] Sawhney, A. S.; Pathak, C. P.; Hubbell, J. A. Macromolecules 1993, 26, 581-587.

[12] Lee, S. H.; Moon, J. J.; West, J. L. Biomaterials 2008, 29, 2962-2968.

[13] Metters, A. T.; Anseth, K. S.; Bowman, C. N. Polymer 2000, 41, 3993-4004.

[14] Papadopoulos, A.; Bichara, D. A.; Zhao, X.; Ibusuki, S.; Randolph, M. A.; Anseth, K. S.; Yaremchuk, M. J. Tissue Eng., Part A 2011, 17, 161-169.

[15] DiRamio, J. A.; Kisaalita, W. S.; Majetich, G. F.; Shimkus, J. M. Biotechnol. Prog. 2005, 21, 1281-1288.

[16] Lee, W. F.; Cheng, T. S. J. Biomater. Sci., Polym. Ed. 2009, 20, 2023-2037.

[17] Sharma, B.; Williams, C. G.; Khan, M.; Manson, P.; Elisseeff, J. H. Plast. Reconstr. Surg. 2007, 119, 112-120.

[18] Poshusta, A. K.; Anseth, K. S. Cells Tissues Organs 2001, 169, 272-278.

[19] Vermonden, T.; Fedorovich, N. E.; van Geemen, D.; Alblas, J.; van Nostrum, C. F.; Dhert, W. J. A.; Hennink, W. E. Biomacromolecules 2008, 9, 919-926.

[20] Censi, R.; Vermonden, T.; Deschout, H.; Braeckmans, K.; di Martino, P.; de Smed, S. C.; van Nostrum, C. F.; Hennink, W. E. Biomacromolecules 2010, 11, 2143-2151.

[21] Censi, R.; Vermonden, T.; van Steenbergen, M. J.; Deschout, H.; Braeckmans, K.; de Smedt, S. C.; van Nostrum, C. F.; di Martino, P.; Hennink, W. E. J. Controlled Release 2009, 140, 230-236.

[22] Hiemstra, C.; Zhou, W.; Zhong, Z. Y.; Wouters, M.; Feijen, J. JACS 2007, 129, 99189926.

[23] Buwalda, S. J.; Dijkstra, P. J.; Calucci, L.; Forte, C.; Feijen, J. Biomacromolecules 2010, 11, 224-232.

[24] Buwalda, S. J.; Calucci, L.; Forte, C.; Dijkstra, P. J.; Feijen, J. Chapter 4, this thesis; University of Twente, 2011.

[25] Buwalda, S. J.; Dijkstra, P. J.; Feijen, J. Chapter 6, this thesis; University of Twente, 2011. 
[26] De Jong, S. J.; de Smedt, S. C.; Wahls, M. W. C.; Demeester, J.; Kettenes-van den Bosch, J. J.; Hennink, W. E. Macromolecules 2000, 33, 3680-3686.

[27] Calucci, L.; Forte, C.; Buwalda, S. J.; Dijkstra, P. J.; Feijen, J. Langmuir 2010, 26, 12890-12896.

[28] Bryant, S. J.; Nuttelman, C. R.; Anseth, K. S. J. Biomater. Sci., Polym. Ed. 2000, 11, 439457.

[29] Piepenbrock, M. O. M.; Lloyd, G. O.; Clarke, N.; Steed, J. W. Chem. Rev. 2010, 110, 1960-2004.

[30] Lin, C. C.; Sawicki, S. M.; Metters, A. T. Biomacromolecules 2008, 9, 75-83.

[31] Moreno-Villoslada, I.; Jofre, M.; Miranda, V.; Chandia, P.; Gonzalez, R.; Hess, S.; Rivas, B. L.; Elvira, C.; San Roman, J.; Shibue, T.; Nishide, H. Polymer 2006, 47, 6496-6500.

[32] Ismail, L. F. M.; Maziad, N. A.; Abo-Farha, S. A. Polym. Int. 2005, 54, 58-64. 


\title{
Chapter 8
}

\section{PEG-PLLA star block copolymer hydrogels crosslinked by metal- ligand coordination}

This chapter has been submitted for publication: Sytze J. Buwalda, ${ }^{a}$ Marijke de Graaff, ${ }^{\text {a }}$ Pieter J. Dijkstra, ${ }^{a}$ and Jan Feijen ${ }^{\mathrm{a}}$ Journal of Polymer Science Part A: Polymer Chemistry 2011, submitted.

${ }^{a}$ Department of Polymer Chemistry and Biomaterials, Faculty of Science and Technology, MIRA Institute for Biomedical Technology and Technical Medicine, University of Twente, P.O. Box 217, 7500 AE Enschede, The Netherlands

\begin{abstract}
The aqueous solution behavior and thermo-reversible gelation properties of pyridine end functionalized poly(ethylene glycol)-poly(L-lactide) star block copolymers (PEG-(PLLA)8-py) in the presence of coordinating transition metal ions was studied. In aqueous solutions the macromonomers self-assembled into micelles and micellar aggregates at low concentrations and formed physically crosslinked, thermo-reversible hydrogels above a critical gel concentration (CGC) of $8 \mathrm{w} / \mathrm{v} \%$. In the presence of transition metal ions like $\mathrm{Cu}(\mathrm{II}), \mathrm{Co}(\mathrm{II})$ or $\mathrm{Mn}(\mathrm{II})$, the aggregate dimensions increased. Above the CGC the gel to sol transition shifted to higher temperatures due to the formation of additional crosslinks from intermolecular coordination complexes between metal ions and pyridine ligands. Furthermore, as an example PEG-(PLLA) 8 -py hydrogels stabilized by $\mathrm{Mn}(\mathrm{II})$-pyridine coordination complexes were more resistant against degradation/dissolution when placed in PBS at $37{ }^{\circ} \mathrm{C}$ compared to hydrogels prepared in water. Importantly, the stabilizing effect of metal-ligand coordination was noticeable at very low $\mathrm{Cu}$ (II) concentrations, which have been reported to be non-cytotoxic in vitro and in vivo. The results show that PEG-(PLLA) 8 -py metallo-hydrogels are appealing materials that may find use in biomedical as well as environmental applications like the removal of heavy metal ions from waste streams.
\end{abstract}




\subsection{Introduction}

There is growing interest in metal containing polymer systems since they constitute a broad class of easily processable materials with unique and valuable properties. ${ }^{1}$ Polymer systems with metal centers may find use as probes, sensors, imaging agents, macromolecular catalysts, artificial enzymes and stimuli-responsive materials. ${ }^{2}$ Most often polymers are used with pendant ligands capable of coordinating transition metal or lanthanide ions. Well-known ligands include pyridine (py), 2,2'-bipyridine (bpy) and 2,2':6',2' -terpyridine (tpy). ${ }^{3-5}$ Due to the stability of metal-ligand complexes, metal-ligand coordination can be employed to crosslink macromonomers into networks. More recently it was envisaged that metallo-gelation can be applied in hydrogels, which are polymer networks based on hydrophilic macromonomers that are able to retain large amounts of water. $^{6-8}$ Pioneering work in the field of metallo-hydrogels was conducted by Chujo et al., who reported on hydrogels based on bpy functionalized poly(oxazoline) crosslinked by Co(II) ions. ${ }^{9}$ The gels were stable at ambient temperatures for several days, but dissolved rapidly at higher temperatures due to a shift in the formation of intermolecular coordination complexes to the entropically favored intramolecular coordination complexes. Recently, Fiore et al. showed that bpy centered poly(ethylene glycol) (PEG) yields hydrogels in aqueous $\mathrm{FeSO}_{4}$ solutions above a polymer concentration of $20 \mathrm{w} / \mathrm{v} \%$ due to the formation of Fe(II)-tris(bpy) complexes. ${ }^{10}$ Exposure to air resulted in dissociation of these complexes as a consequence of oxidation of $\mathrm{Fe}(\mathrm{II})$ to $\mathrm{Fe}(\mathrm{III})$. When the bpy centered PEG was endcapped with methacrylate groups and subsequently UV irradiated, covalently crosslinked networks were obtained. Upon incubation of these networks in $\mathrm{NiCl}_{2}$ or $\mathrm{CoCl}_{2}$ solutions, the metal ions became uniformly distributed throughout the gel, indicating that the bpy sites remained available for metal coordination. The same group reported on linear PLA-PEGPLA triblock copolymers functionalized with a bpy ligand. ${ }^{11}$ Nanoparticles could be prepared by precipitation of the polymer in water using $\mathrm{FeCl}_{2}$ in $\mathrm{DMSO}$, but gelation properties were not studied. Dynamic light scattering and transmission electron microscopy confirmed the presence of star-like Fe(II)-polymer complexes of 40-80 nm in diameter. Vermonden et al. investigated the aggregation of short PEG chains endcapped with 2,6- dicarboxypyridine groups in aqueous solutions containing metal ions. ${ }^{12}$ The presence of $\mathrm{Zn}$ (II) ions or transition metal ions like Ni(II) resulted in linear polymer structures, whereas neodymium(III) or lanthanum(III) ions yielded threedimensional polymer networks. This was ascribed to the larger size of the lanthanide ions which allows for the coordination of additional ligands compared to $4^{\text {th }}$ period transition metal ions. At high concentrations, the polymer networks behaved as viscoelastic materials that were thermoreversible. ${ }^{13}$ The group of Schubert prepared tpy containing dextrans which form assemblies in the presence of $\mathrm{Fe}(\mathrm{II})$ or $\mathrm{Ru}(\mathrm{II})$ ions. ${ }^{14}$ Depending on the degree of tpy functionalization, a shift from inter- to intramolecular crosslinking was observed. Comb type copolymers could be obtained by the formation of coordination complexes of bpy functionalized dextrans and mono-bpy functionalized 
PEGs using $\mathrm{Ru}(\mathrm{II})$, but gelation studies were not reported. The same group also reported on the association behavior of poly(ethylene oxide)-poly(propylene oxide)-poly(ethylene oxide) (PEOPPO-PEO, commercially known as Pluronics) endcapped with tpy ligands in water. ${ }^{15}$ It was found that the addition of 0.5 equivalents of $\mathrm{Ni}(\mathrm{II})$ ions relative to tpy groups induced the formation of bis(tpy)-Ni(II) complexes and disrupted initially formed hydrophobic tpy aggregates. Addition of $\mathrm{Ni}(\mathrm{II})$ ions to an $18 \mathrm{w} / \mathrm{v} \%$ solution of tpy functionalized Pluronics resulted in the formation of a hydrogel but no gel properties or temperature dependent gelation were described.

Previously we found that PEG-(PLLA 12$)_{8}$ star block copolymers yield thermo-reversible hydrogels with good mechanical properties. ${ }^{16}$ These materials, which are biodegradable through hydrolysis of PLLA ester bonds, do show a limited gel stability in aqueous environments due to the rapid degradation and/or dissolution of the polymers in these physically crosslinked networks. Based on these findings, dual type crosslinked networks were expected to form more robust hydrogels. In this paper we describe the synthesis and aqueous solution properties of pyridine functionalized star block copolymers (PEG-(PLLA) 8 -py). These star block copolymers were cocrosslinked in the presence of (transition) metal ions and the resulting mechanical and degradation properties of these novel PEG-(PLLA) 8 -py metallo-hydrogels were determined.

\subsection{Experimental section}

Materials. Hydroxyl terminated 8-armed poly(ethylene glycol) (PEG-(OH) $8, M_{n}$, NMR $=23800$ $\mathrm{g} / \mathrm{mol}$ ) was purchased from Jenkem (Allen, Texas, USA) and purified before use by dissolution in dichloromethane and precipitation in cold diethyl ether. PEG- $(\mathrm{OH})_{8}$ was converted in PEG- $\left(\mathrm{NH}_{2}\right)_{8}$ as described previously. ${ }^{16}$ L-lactide was obtained from Purac (Gorinchem, the Netherlands). Iron(III) chloride, sodium chloride, copper(II) chloride, zinc chloride, cobalt chloride, manganese chloride, copper(II) sulfate, methanesulfonyl chloride (mesyl chloride), tin(II) 2-ethylhexanoate (stannous octoate), triethylamine (TEA), $25 \%$ aqueous ammonia solution, succinic anhydride and N-hydroxysuccinimide (NHS) were from Sigma-Aldrich (St Louis, Missouri, USA). 4Aminopyridine and N,N'dicyclohexylcarbodiimide were purchased from Acros (Geel, Belgium). 4Dimethylaminopyridine was bought from Merck (Darmstadt, Germany). Toluene, tetrahydrofuran, diethyl ether, methanol and dichloromethane were all from Biosolve (Valkenswaard, the Netherlands). Dichloromethane and tetrahydrofuran were dried over calcium hydride, whereas TEA and toluene were dried over potassium hydroxide and sodium, respectively. All solvents were distilled prior to use.

The 8-armed poly(ethylene glycol)-poly(L-lactide) star block copolymer with a degree of polymerization of 12 lactyl units per PLLA arm (PEG-(PLLA) 8 ) was synthesized by ring opening polymerization of L-lactide in toluene at $110{ }^{\circ} \mathrm{C}$. Amine terminated 8-armed star PEG (PEG- 
$\left.\left(\mathrm{NH}_{2}\right)_{8}\right)$ and stannous octoate were used as initiator and catalyst, respectively. This procedure was described in detail previously. ${ }^{16}$

Synthesis. The 8-armed poly(ethylene glycol)-poly(lactide) star block copolymers with pyridine end groups (PEG-(PLLA) 8 -py) were synthesized in a three-step procedure starting from PEG(PLLA)8. First the PLLA hydroxyl end groups were reacted with succinic anhydride to yield carboxylic acid end groups, which were subsequently activated by conversion to their NHS esters. Finally, pyridine end groups were obtained by reaction of 4-aminopyridine with the activated carboxylic acid groups.

A typical procedure for the synthesis of the PEG-(PLLA) 8 star block copolymer with carboxylic acid end groups (PEG-(PLLA) $)_{8}-\mathrm{c}$ ) was as follows. To a solution of PEG-(PLLA) 8 (12 g, 0.39 $\mathrm{mmol})$ in $125 \mathrm{ml}$ of dichloromethane, succinic anhydride (0.47 g, $4.7 \mathrm{mmol})$, 4dimethylaminopyridine $(0.29 \mathrm{~g}, 2.3 \mathrm{mmol})$ and TEA $(0.44 \mathrm{ml}, 3.2 \mathrm{mmol})$ were added. The reaction was allowed to proceed for $24 \mathrm{~h}$ at room temperature in a nitrogen atmosphere. The reaction mixture was filtered and concentrated under reduced pressure. The product was purified by precipitation in a large excess of a cold mixture of diethyl ether and methanol $(10 / 1 \mathrm{v} / \mathrm{v})$. PEG(PLLA) $)_{8}$-c was obtained by filtration and dried overnight in vacuo at room temperature over phosphorus pentoxide. In the second step of the synthesis procedure, PEG-(PLLA) 8 -c $(3.0 \mathrm{~g}, 0.10$ mmol) was dissolved in $60 \mathrm{ml}$ of dichloromethane. Subsequently NHS (0.12 g, $1.1 \mathrm{mmol})$ was added, followed by the addition of N,N'dicyclohexylcarbodiimide $(0.28 \mathrm{~g}, 1.4 \mathrm{mmol})$. The reaction mixture was stirred at room temperature for $24 \mathrm{~h}$ in a nitrogen atmosphere. The mixture was filtered until clear, concentrated under reduced pressure and precipitated in a large excess of a cold mixture of diethyl ether and methanol (20/1 v/v). PEG-(PLLA) $)_{8}-\mathrm{c}-\mathrm{NHS}$ was obtained by filtration and dried under reduced pressure in the presence of phosphorus pentoxide. In the final step of the synthesis, PEG-(PLLA) $)_{8}$-c-NHS (1.0 g, $\left.31 \mu \mathrm{mol}\right)$ was dissolved in $50 \mathrm{ml}$ of tetrahydrofuran. After addition of 4-aminopyridine $(0.23 \mathrm{~g}, 2.5 \mathrm{mmol})$, the reaction was allowed to proceed for $3 \mathrm{~d}$ at room temperature in a nitrogen atmosphere. PEG-(PLLA) 8 -py was purified by precipitation in a large excess of cold diethyl ether and after filtration dried under reduced pressure. ${ }^{1} \mathrm{H}$ NMR (DMSO- $\left.\mathrm{d}_{6}\right)$ : $\delta=7.98$ (m, pyridine H-2, H-2'), 7.53 (s, CONHC), 6.44 (d, pyridine H-3, H-3'), 5.93 (s, $\mathrm{CH}_{2} \mathrm{NHCO}$ ), 5.20 (m, $\mathrm{CH}_{3} \mathrm{CH}$ ), 4.97 (m, $\mathrm{CH}_{3} \mathrm{CHOCO}$ ) 3.51 (PEG protons), 2.55-2.70 (m, $\left.\mathrm{OCOCH}_{2} \mathrm{CH}_{2} \mathrm{CONH}\right), 1.47$ (d, $\left.\mathrm{CH}_{3} \mathrm{CH}\right), 1.34$ (d, $\left.\mathrm{CH}_{3} \mathrm{CHOCO}\right)$.

Characterization. ${ }^{1} \mathrm{H}$ NMR $(300 \mathrm{MHz})$ spectra were recorded on a Varian Inova $300 \mathrm{NMR}$ spectrometer. Polymers were dissolved in $\mathrm{CDCl}_{3}, \mathrm{D}_{2} \mathrm{O}$ or DMSO- $\mathrm{d}_{6}$ at a concentration of $15 \mathrm{mg} / \mathrm{ml}$. 
Aqueous solution properties. Dynamic light scattering (DLS) of the PEG-(PLLA) 8 -py star block copolymer was performed to determine aggregate sizes in distilled water and in $0.25 \mathrm{M} \mathrm{MnCl}_{2}$ solution at a polymer concentration of $0.5 \mathrm{w} / \mathrm{v} \%$. Experiments were carried out at $25{ }^{\circ} \mathrm{C}$ using a Malvern Nano ZS, a laser wavelength of $633 \mathrm{~nm}$ and a scattering angle of $173^{\circ}$.

Gel properties. The vial tilting method was used to determine the temperature dependent

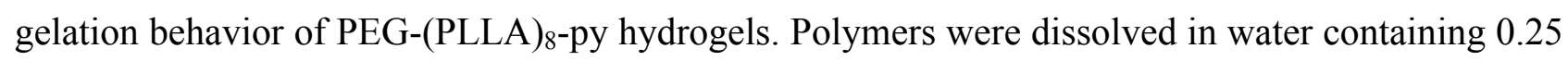
$\mathrm{M} \mathrm{MnCl}_{2}$ or $\mathrm{NaCl}$. The temperature dependent gelation behavior was studied in the range of 20-80 ${ }^{\circ} \mathrm{C}$ with temperature increments of $5{ }^{\circ} \mathrm{C}$. At each temperature, the sample was allowed to equilibrate for $10 \mathrm{~min}$. If there was no flow after tilting the vials $90^{\circ}$ for $1 \mathrm{~min}$, the sample was regarded as a gel; otherwise, it was regarded as a sol.

Oscillatory rheology experiments were performed to determine the mechanical properties of the PEG-(PLLA) $)_{8}$-py hydrogels which were prepared using $0.25 \mathrm{M} \mathrm{FeCl}_{3}, \mathrm{NaCl}, \mathrm{CuCl}_{2}, \mathrm{ZnCl}_{2}, \mathrm{CuSO}_{4}$, $\mathrm{CoCl}_{2}$ or $\mathrm{MnCl}_{2}$ solution. The storage $\left(\mathrm{G}^{\prime}\right)$ and loss $\left(\mathrm{G}^{\prime \prime}\right)$ modulus of hydrogels were monitored for 3 min at $20{ }^{\circ} \mathrm{C}$ on an Anton-Paar Physica MCR 301 rheometer. Experiments were performed using a flat plate measuring geometry (diameter $25 \mathrm{~mm}$, gap $0.3 \mathrm{~mm}$ ) utilizing a strain of $0.5 \%$ and a frequency of $1 \mathrm{~Hz}$.

Degradation/dissolution experiments were performed to determine the stability of the PEG(PLLA) 8 -py hydrogels which were prepared using $0.25 \mathrm{M} \mathrm{MnCl}_{2}$ or $\mathrm{NaCl}$ solution. On top of 0.5 $\mathrm{ml}$ of hydrogel, $3 \mathrm{ml}$ of PBS was placed and the samples were kept at $37{ }^{\circ} \mathrm{C}$. To prevent bacterial growth, $0.02 \mathrm{w} / \mathrm{v} \% \mathrm{NaN}_{3}$ was added to the buffer solution. At regular time intervals, the vials were tilted $90^{\circ}$. The degradation/dissolution time was defined as the number of days until the sample became fully solubilized. Degradation/dissolution experiments were performed in duplicate. 


\subsection{Results and discussion}

Synthesis of PEG-PLLA star block copolymers with pyridine end groups. PEG-PLLA star block copolymers with pyridine end groups were prepared starting from PEG-(OH) 8 (Figure 1). PEG-(PLLA $\left.{ }_{12}\right)_{8}$ star block copolymers with 12 lactyl units per arm and hydroxyl end groups were reacted with succinic anhydride to afford polymers with carboxylic acid end groups in high yield. The carboxylic acid groups were subsequently converted in their N-hydroxysuccinimide active esters and reacted with 4-aminopyridine to give the pyridine end functionalized block copolymers (Figure 1).

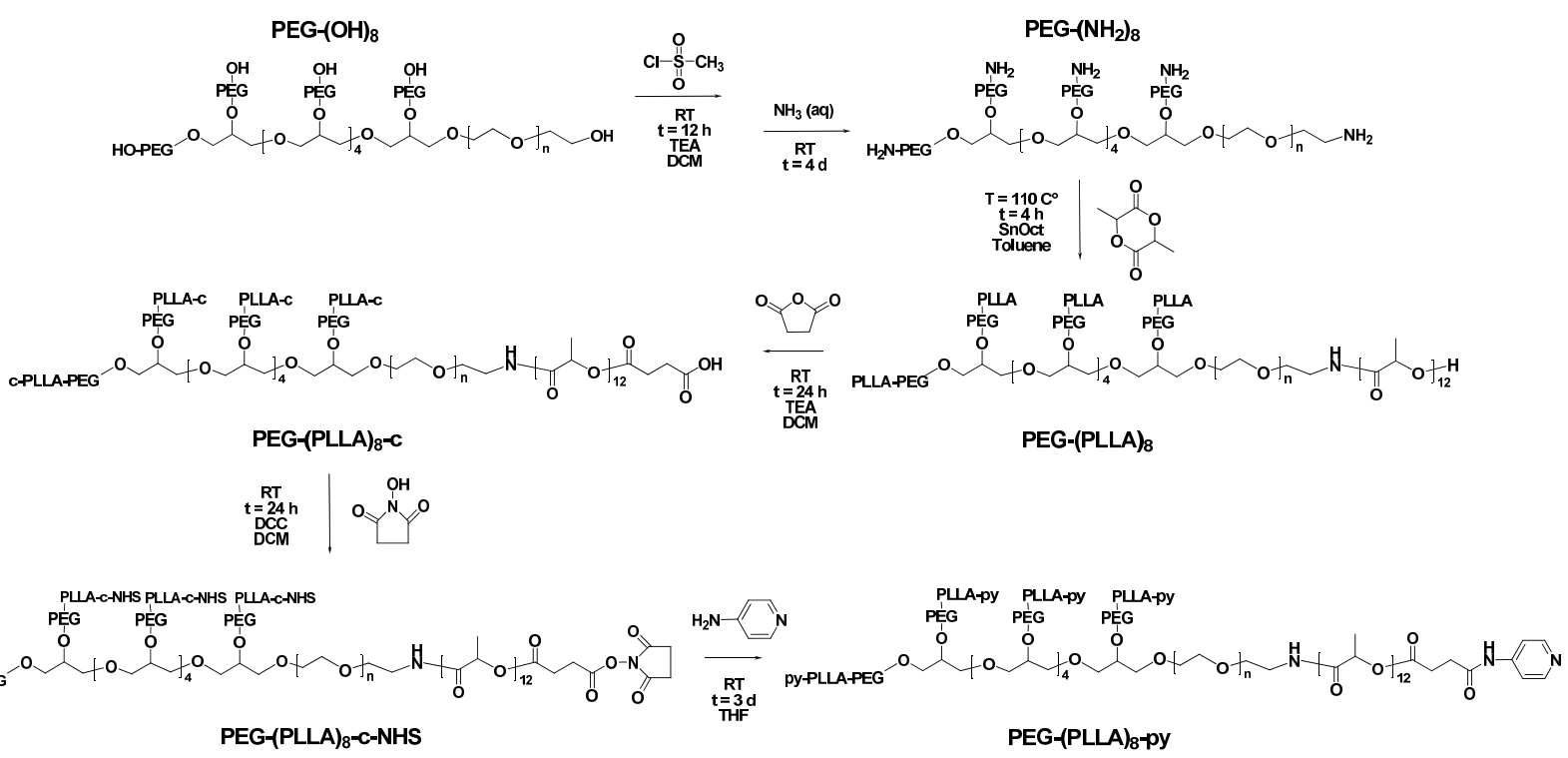

Figure 1. Synthesis scheme for the preparation of PEG-(PLLA) 8 -py star block copolymers.

In the spectrum of PEG-(PLLA) $)_{8}$-c a broad signal at 2.6-2.8 ppm confirms the presence of the succinic methylene protons (Figure 2). Upon conversion of the carboxylic acid groups in their Nhydroxysuccinimide active esters the succinic methylene protons (e) partially shift downfield and a peak at $2.83 \mathrm{ppm}$ appears corresponding to the succinimide protons. 

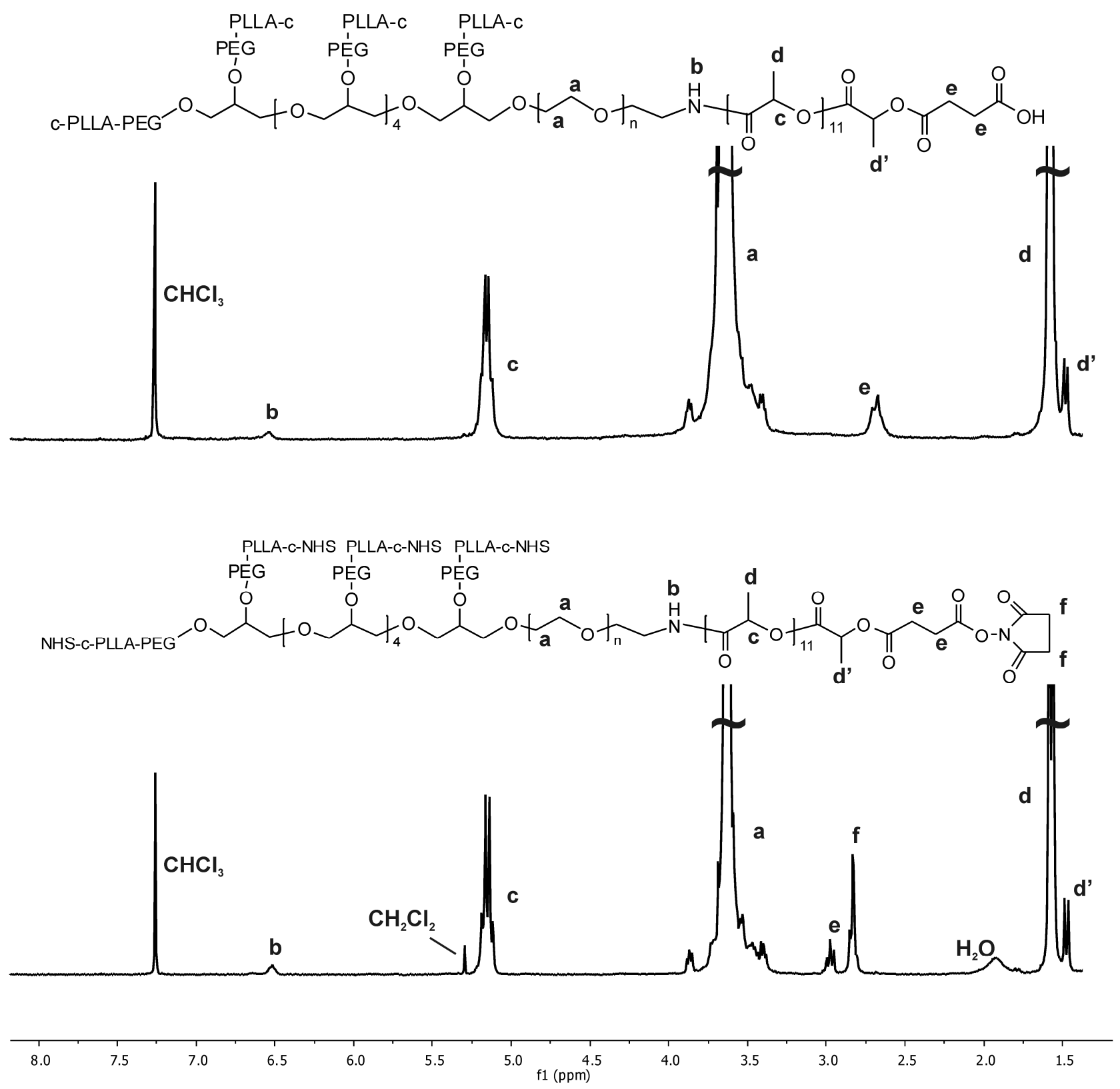

Figure 2. ${ }^{1} \mathrm{H}$ NMR spectra of PEG-(PLLA) $)_{8}$-c-NHS (bottom) and PEG-(PLLA) 8 -c (top) in $\mathrm{CDCl}_{3}$. Unassigned peaks belong to spinning side bands. 
In the ${ }^{1} \mathrm{H}$ NMR spectrum (DMSO-d ${ }_{6}$ ) of PEG-(PLLA) 8 -py signals are visible at 7.98 and 6.44 ppm corresponding to the pyridine protons (Figure 3). Moreover, a signal at $7.53 \mathrm{ppm}$ shows the linking amide group between the pyridine group and PEG-PLLA star block copolymer. Based on the integral ratio of signals $\mathbf{h}$ and $\mathbf{c}$, the upfield shift of the succinic methylene protons (e) as well as the absence of signals relating to residual NHS groups at $2.83 \mathrm{ppm}$, it was concluded that all 8 arms of PEG-(PLLA) 8 -py were functionalized with a pyridine group.

In the ${ }^{1} \mathrm{H}$ NMR spectrum of PEG-(PLLA) $)_{8}$-py dissolved in $\mathrm{D}_{2} \mathrm{O}$, the relative intensity of the PLA signals decreases to a large extend because the hydrophobic PLA blocks are condensed into hydrophobic domains in an aqueous environment (Figure 3). The observable PLA protons (30\% of the total PLA units) correspond to PLA chains dangling in water and to the more mobile chains in hydrophobic domains. ${ }^{17}$ Although the para-amide substituted pyridine groups are close to the PLLA blocks these groups are well detectable. It may be concluded that the end groups are present in the more hydrophilic regions of the aggregates. This conclusion is supported by the ${ }^{1} \mathrm{H}$ NMR spectral data of the block copolymer dissolved in $\mathrm{CDCl}_{3}$. As seen in Figure 3 (top) no signals appear for the pyridine protons meaning they have a low mobility possibly due to aggregation. Moreover the amide protons could also not be detected. These results indicate that a high number of pyridine ligands are available in the aqueous phase to participate in the formation of metal ion-pyridine complexes. 

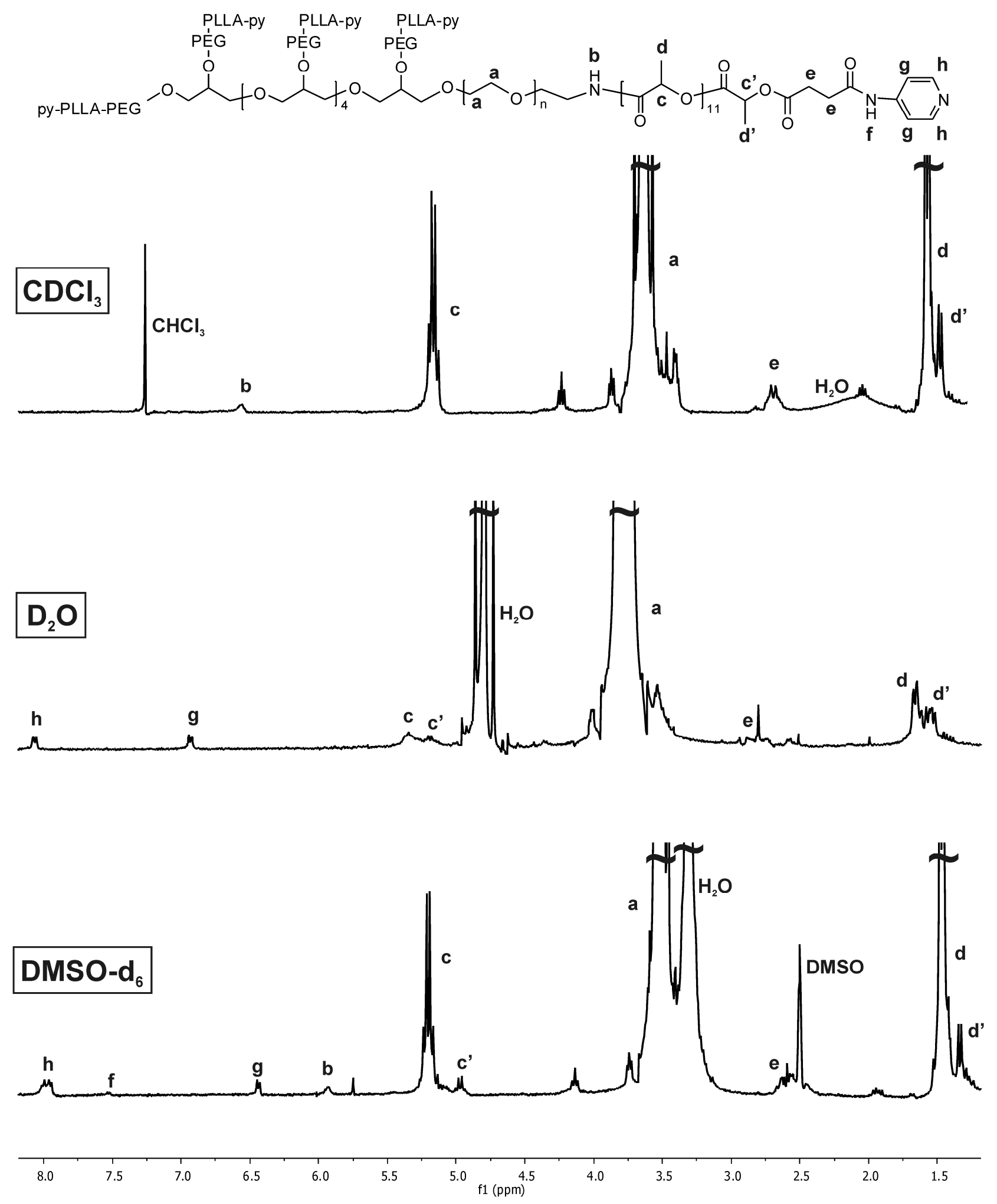

Figure 3. ${ }^{1} \mathrm{H}$ NMR spectra of PEG-(PLLA) $)_{8}$-py in DMSO- $\mathrm{d}_{6}$ (bottom), $\mathrm{D}_{2} \mathrm{O}$ (middle) and $\mathrm{CDCl}_{3}$ (top). Unassigned peaks belong to spinning side bands.

Rheology. Physically crosslinked PEG-(PLLA 12$)_{8}$ star block copolymers show thermo-reversible gel- sol transitions above a critical gel concentration (CGC) of $\sim 10 \mathrm{w} / \mathrm{v} \%{ }^{16}$ The CGC of PEG(PLLA) $)_{8}$-py was found at a similar value of approximately $8 \mathrm{w} / \mathrm{v} \%$ (Figure 6). Because the 
pyridine groups in PEG-(PLLA) $)_{8}$-py are in the hydrophilic regions it is possible to form coordination complexes with transition metal ions, which will lead to additional crosslinks and improved mechanical properties. For initial experiments $\mathrm{CuCl}_{2}$ was selected because $\mathrm{Cu}(\mathrm{II})$ ions were previously shown to form coordination complexes with pyridine type ligands. ${ }^{3}$ Oscillatory rheology measurements were performed on the polymer dissolved in a $0.25 \mathrm{M} \mathrm{CuCl}_{2}$ solution.

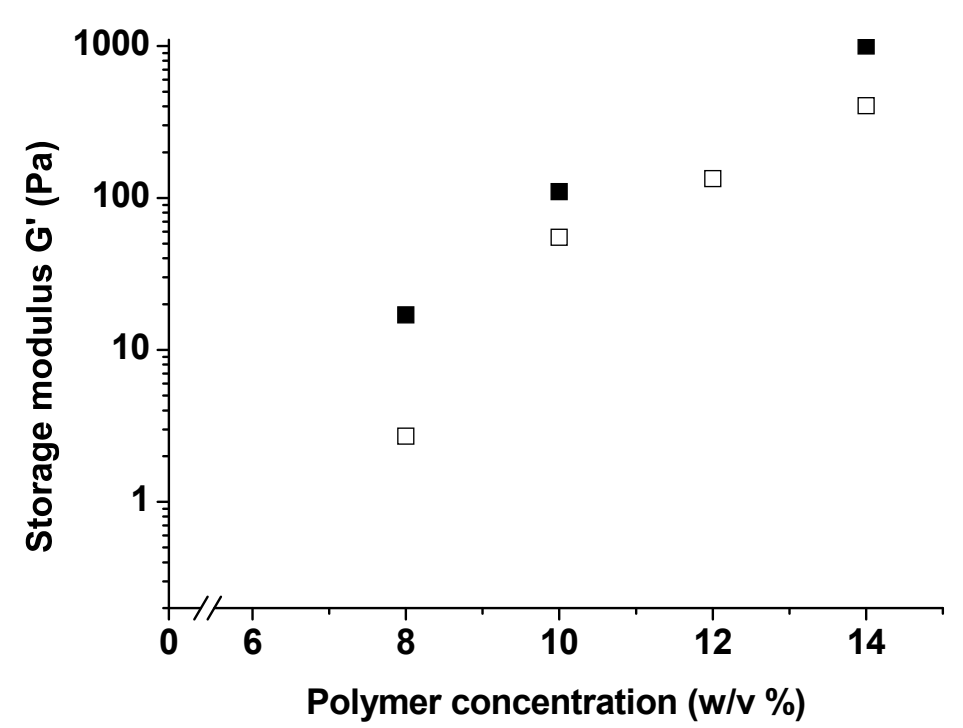

Figure 4. Storage modulus (G') at $20{ }^{\circ} \mathrm{C}$ versus concentration for PEG-(PLLA) $)_{8}$-py hydrogels prepared in $0.25 \mathrm{M} \mathrm{CuCl}_{2}$ solution ( $\left.\mathbf{\square}\right)$ and in distilled water $(\square)$.

Over the entire concentration range, the storage modulus is higher for PEG-(PLLA) 8 -py hydrogels prepared in $0.25 \mathrm{M} \mathrm{CuCl}_{2}$ solution compared to hydrogels prepared in water (Figure 4). These data are evidence of the formation of intermolecular coordination complexes between metal ions and pyridine ligands resulting in the formation of additional crosslinks. It cannot be excluded that also intramolecular complexes are formed. The increase in $G^{\prime}$ with polymer concentration (Figure 4) can be ascribed to the formation of a more densely crosslinked network. As expected the effect of the additional metal-pyridine crosslinks is more noticeable at lower concentrations where the physical crosslink density is relatively low.

The nature of both the metal ion and counter ion may have a profound effect on the metalpyridine coordination complex formed and its association constant. ${ }^{18}$ The storage modulus G' of 10 w/v \% PEG-(PLLA) 8 -py hydrogels prepared using $0.25 \mathrm{M} \mathrm{CuCl}_{2}, \mathrm{ZnCl}_{2}, \mathrm{CuSO}_{4}, \mathrm{CoCl}_{2}$ or $\mathrm{MnCl}_{2}$ solutions was determined with oscillatory rheology measurements at $20{ }^{\circ} \mathrm{C}$ (Table 1). PEG(PLLA) 8 -py hydrogels (10 w/v \%) prepared in $0.25 \mathrm{M} \mathrm{FeCl}_{3}, \mathrm{NaCl}$ or water were used as controls. Both $\mathrm{Zn}$ ions and the transition metal ions $\mathrm{Cu}(\mathrm{II}), \mathrm{Co}(\mathrm{II})$ and $\mathrm{Mn}$ (II) exhibit a higher storage modulus compared to a PEG-(PLLA) 8 -py hydrogel prepared in water, indicating that coordination of these metal ions with the pyridine ligands leads to a higher physical crosslink density. Moreover changing the anion from chloride to sulfate $\left(\mathrm{CuCl}_{2}\right.$ vs. $\left.\mathrm{CuSO}_{4}\right)$ results in a significant increase in G' 
from 110 to $225 \mathrm{~Pa}$. The storage moduli of the hydrogels prepared in $\mathrm{NaCl}$ or $\mathrm{FeCl}_{3}$ solution are lower compared to the hydrogel prepared in distilled water. This may be attributed to the reduction in the effective hard sphere volume of the micelles (micellar aggregates) in a salt solution, which is a poorer solvent than water. ${ }^{19}$ The results confirm that the pyridine ligands can form coordination complexes with a range of metal ions. ${ }^{20}$

Table 1. Storage modulus (G') at $20{ }^{\circ} \mathrm{C}$ for $10 \mathrm{w} / \mathrm{v} \%$ PEG-(PLLA) 8 -py hydrogels prepared in various $0.25 \mathrm{M}$ salt solutions.

\begin{tabular}{|c|c|c|c|c|c|c|c|c|}
\hline Salt & $\mathrm{FeCl}_{3}$ & $\mathrm{NaCl}$ & No salt & $\mathrm{CuCl}_{2}$ & $\mathrm{ZnCl}_{2}$ & $\mathrm{CuSO}_{4}$ & $\mathrm{CoCl}_{2}$ & $\mathrm{MnCl}_{2}$ \\
\hline $\mathrm{G}^{\prime}(\mathrm{Pa})$ & 10 & 20 & 55 & 110 & 110 & 225 & 250 & 285 \\
\hline
\end{tabular}

No significant effect of the salt concentration on the mechanical properties was found, as $10 \mathrm{w} / \mathrm{v}$ \% PEG-(PLLA) 8 -py hydrogels prepared in $0.025 \mathrm{M}, 0.25 \mathrm{M}$ or $4 \mathrm{M}_{\text {aqueous }} \mathrm{CuCl}_{2}$ solutions all yielded a similar storage modulus of approximately $100 \mathrm{~Pa}$. Importantly a concentration of $0.025 \mathrm{M}$ $\mathrm{Cu}(\mathrm{II})$ was previously reported to be non-cytotoxic for fibroblasts. ${ }^{21}$

PEG-(PLLA) 8 -py hydrogels $\left(10 \mathrm{w} / \mathrm{v} \%\right.$ ) prepared in a $0.25 \mathrm{M} \mathrm{MnCl}_{2}$ solution exhibit the highest storage modulus, indicative of a higher association constant of the $\mathrm{Mn}$ (II) coordination complex with pyridine ligands. In the following sections the self-assembly of PEG-(PLLA) 8 -py in $0.25 \mathrm{M}$ $\mathrm{MnCl}_{2}$ solutions as well as the temperature sensitive gelation behavior and the hydrogel degradation are presented.

Self-assembly in aqueous solutions and gelation behavior. PEG-(PLLA 12$)_{8}$ star block copolymers self-assemble at low concentrations in water to form micelles and aggregates with a hydrophobic PLA core and a hydrophilic PEG shell. ${ }^{22}$ In the periphery of the aggregates also PLA domains are present and free PLA chains dangling in water. ${ }^{17}$ Using dynamic light scattering (DLS) the aggregate sizes and aggregate size distributions were determined for PEG-(PLLA) 8 -py star block copolymers dissolved at a low concentration $(0.5 \mathrm{w} / \mathrm{v} \%)$ in water and in a $0.25 \mathrm{M} \mathrm{MnCl}_{2}$ solution (Figure 5). 


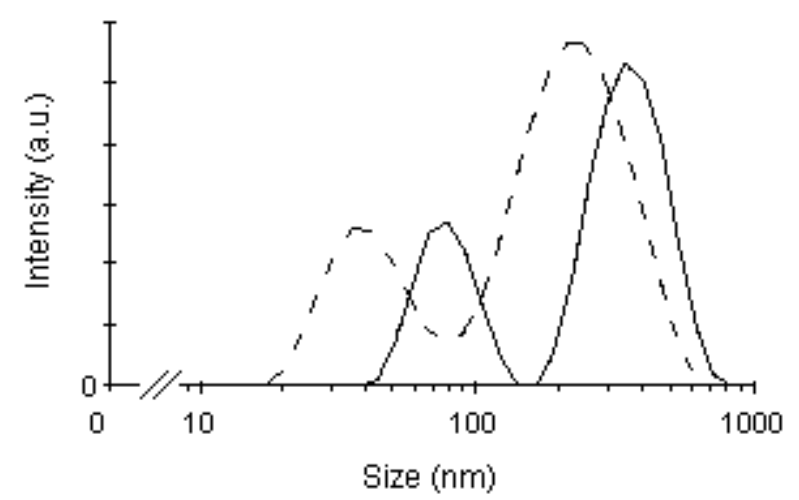

Figure 5. Aggregate size distributions of PEG-(PLLA) 8 -py star block copolymers in distilled water (dashed line) and in $0.25 \mathrm{M} \mathrm{MnCl}_{2}$ solution (solid line) at $25^{\circ} \mathrm{C}$ (polymer concentration of $0.5 \mathrm{w} / \mathrm{v}$ $\%)$.

In water, the block copolymer forms small aggregates with an average diameter of 30-40 nm, close to the typical size of micelles, as well as larger aggregates with a diameter of $\sim 250 \mathrm{~nm}$, which may result from secondary aggregation of micelles. ${ }^{23}$ These values are in a similar range as compared to those of the non-functionalized PEG-(PLLA 12$)_{8} \cdot{ }^{16}$ The presence of Mn(II) ions in an aqueous solution of PEG-(PLLA) 8 -py results in a clear increase in aggregate dimensions (Figure 5). In previous research it was shown that micelles and micellar aggregates of PEG-(PLLA) 8 star block copolymers contain different types of hydrophobic domains of PLLA blocks and even PLLA chains dangling in water. ${ }^{17} \mathrm{H}$ NMR measurements using $\mathrm{D}_{2} \mathrm{O}$ suggested that the pyridine ligands which are covalently attached to the end of the PLLA blocks are located in the aqueous phase and have a high mobility (Figure 3). Therefore, the increased aggregate dimensions are most likely caused by the formation of intermolecular Mn(II)-pyridine complexes.

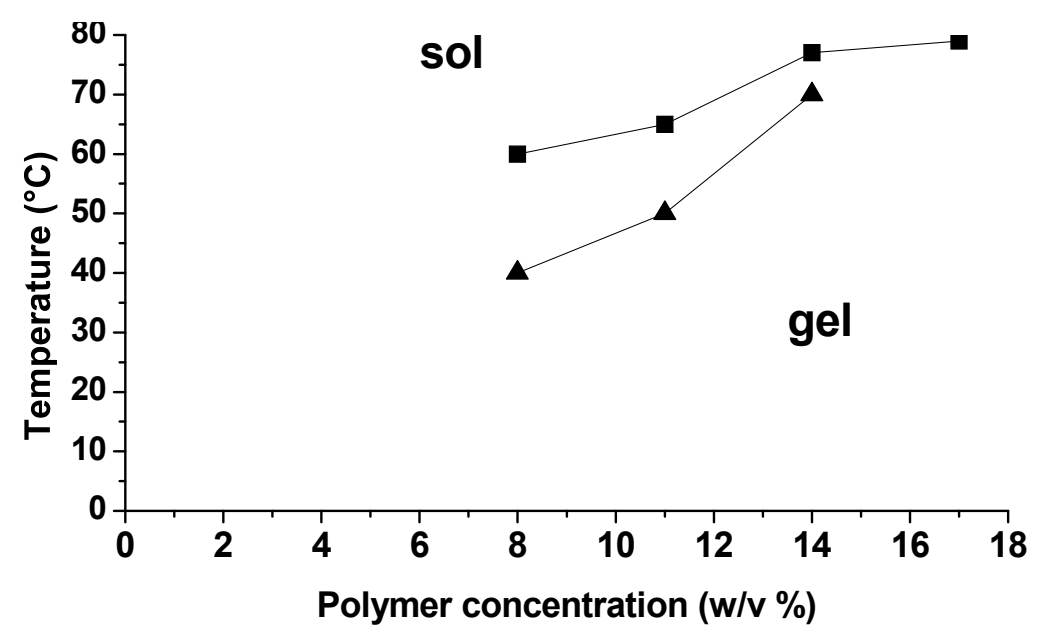

Figure 6. Gel-sol transition temperatures of PEG-(PLLA) 8 -py star block copolymers in aqueous solution determined with the vial tilting method: $0.25 \mathrm{M} \mathrm{MnCl}_{2}(\mathbf{\square})$ and $0.25 \mathrm{M} \mathrm{NaCl}$ solution ( $\mathbf{\Delta}$ ). 
The thermo-reversible gelation properties of PEG-(PLLA) $)_{8}$-py dissolved in $0.25 \mathrm{M} \mathrm{MnCl}_{2}$ or $\mathrm{NaCl}$ solutions were investigated with the vial tilting method using a temperature range of 20 to 80 ${ }^{\circ} \mathrm{C}$ (Figure 6). Above the CGC of $8 \mathrm{w} / \mathrm{v} \%$ PEG-(PLLA) $)_{8}$-py forms hydrogels as a result of PEG entanglements and interaggregate PLA bridging. In the presence of $\mathrm{Mn}(\mathrm{II})$ ions, additional metalpyridine crosslinks are formed (Figure 7). The formed gels exhibit a transition from the gel to the sol state upon a temperature increase due to dehydration of the PEG, which causes the aggregates to shrink resulting in decreased interactions between the aggregates. This transition is fully reversible upon cooling. Replacing $\mathrm{Na}(\mathrm{I})$ ions for $\mathrm{Mn}$ (II) ions leads to gel to sol transitions at higher temperatures (Figure 6) as a result of the additional metal-pyridine crosslinks. The shift to larger aggregates in PEG-(PLLA) $)_{8}$-py / $\mathrm{MnCl}_{2}$ solutions compared to those present in water (Figure 5) may also be a reason for the shift of the boundary curve to higher temperatures. ${ }^{16}$ An increase in polymer concentration shifts the gel to sol transition to higher temperatures as a result of an increased number of physical crosslinks (Figure 6).

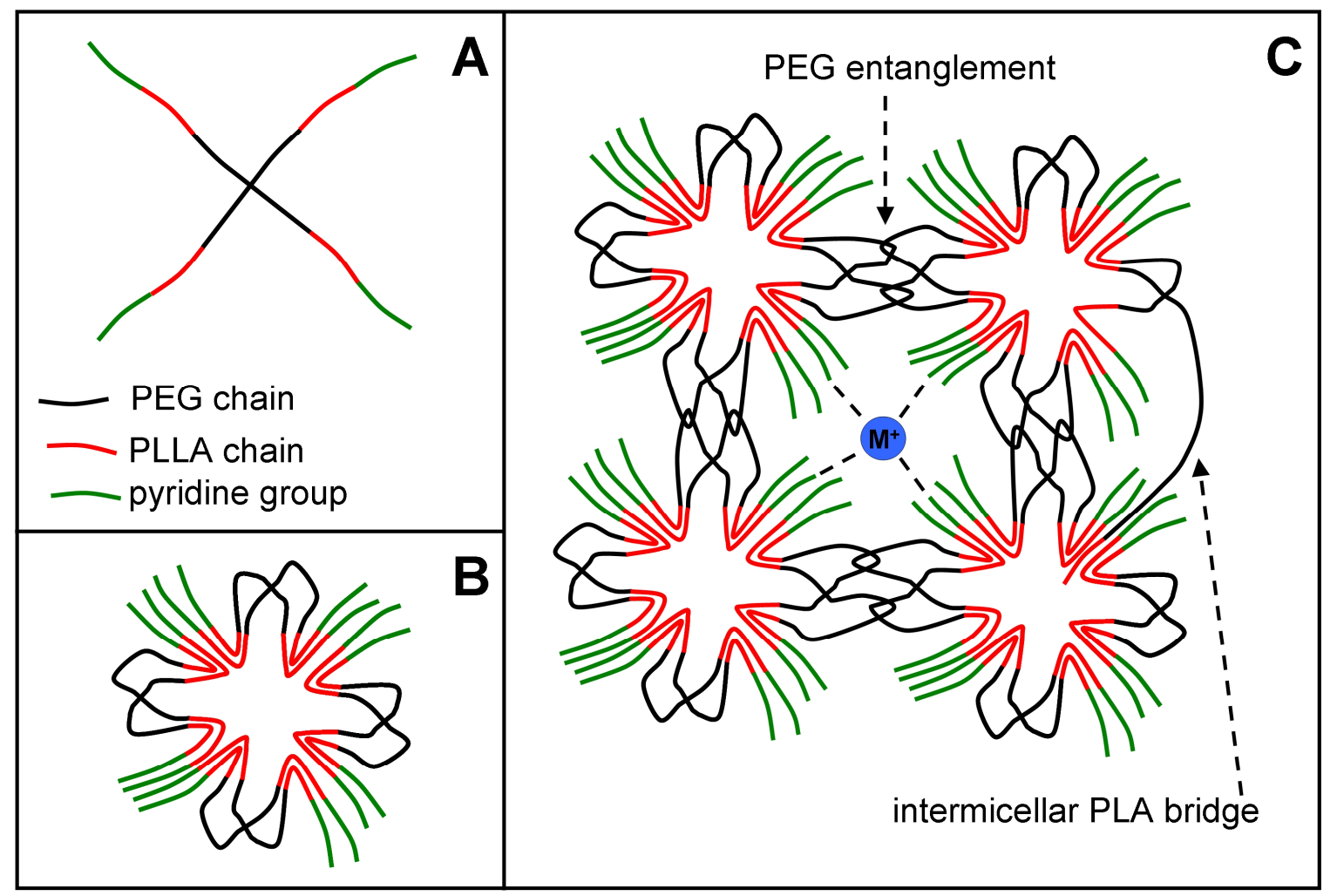

Figure 7. Schematic representation of a PEG-(PLLA)-py star block copolymer (A), a PEG-(PLLA)py micelle in aqueous solution (B) and a PEG-(PLLA)-py hydrogel in the presence of metal ions (C). For clarity, PEG-(PLLA)-py star block copolymer is drawn with 4 arms.

Hydrogel degradation/dissolution. The in vitro stability of PEG-(PLLA) 8 -py hydrogels, prepared in $0.25 \mathrm{M} \mathrm{NaCl}$ or $\mathrm{MnCl}_{2}$, was investigated by placing PBS on top of the hydrogel samples at $37^{\circ} \mathrm{C}$ and determining the number of days until the samples became fully solubilized. It 
was observed that a $12 \mathrm{w} / \mathrm{v} \%$ PEG-(PLLA) 8 -py / $\mathrm{NaCl}$ hydrogel completely solubilized within $1 \mathrm{~d}$. A $12 \mathrm{w} / \mathrm{v} \%$ PEG-(PLLA) 8 -py / $\mathrm{MnCl}_{2}$ hydrogel exhibited a significantly improved stability, and complete dissolution was only observed at day 3. Physical PEG-PLA type hydrogels generally loose their structural integrity because the close packing of micelles and aggregates is affected due to the concentration difference of the copolymer in the hydrogel and supernatant buffer. The stabilizing interactions between $\mathrm{Mn}$ (II) ions and pyridine ligands probably lead to less facile disruption of the micellar and aggregate packing. Increasing the concentration of the PEG-(PLLA) $8^{-}$ py / $\mathrm{MnCl}_{2}$ hydrogel from 12 to $16 \mathrm{w} / \mathrm{v} \%$ extended the dissolution time from 3 to $10 \mathrm{~d}$. A higher physical crosslink density is most likely the reason for this prolonged stability.

\subsection{Conclusions}

Coordination complexation of transition metal ions and PEG-(PLLA)s-py star block copolymers with pendant pyridine ligands has a profound effect on the solution and thermo-reversible gelation properties of these materials. Oscillatory rheology measurements confirmed that transition metal ions interact efficiently with pyridine ligands to form metal-ligand complexes with a 5 to 10 times higher storage modulus than in $\mathrm{NaCl}$ solutions at similar concentrations. Dynamic light scattering experiments showed that at low concentrations, aqueous solutions of PEG-(PLLA) -py $_{\text {-ponsist of }}$ micelles and aggregates. At higher concentrations, thermo-responsive gels were obtained, exhibiting a reversible gel to sol transition upon temperature increase. The presence of Mn(II) ions resulted in increased aggregate dimensions, a larger gel window and increased in vitro stability due to the coordination of pyridine ligands around the metal centers, thus introducing additional crosslinks. The possibility to modulate hydrogel properties by metal ligation makes the PEG(PLLA) $)_{8}$-py hydrogels versatile materials which may find use in a variety of applications.

\subsection{References}

[1] Whittell, G. R.; Hager, M. D.; Schubert, U. S.; Manners, I. Nat. Mater. 2011, 10, 176188.

[2] Pfister, A.; Fraser, C. L. Biomacromolecules 2006, 7, 459-468.

[3] Lewis, A. L.; Miller, J. D. Polymer 1993, 34, 2453-2457.

[4] Fustin, C. A.; Guillet, P.; Schubert, U. S.; Gohy, J. F. Adv. Mater. 2007, 19, 1665-1673.

[5] Shunmugam, R.; Gabriel, G. J.; Aamer, K. A.; Tew, G. N. Macromol. Rapid Commun. 2010, 31, 784-793.

[6] Zisch, A. H.; Lutolf, M. P.; Hubbell, J. A. Cardiovasc. Pathol. 2003, 12, 295-310.

[7] Peppas, N. A.; Bures, P.; Leobandung, W.; Ichikawa, H. Eur. J. Pharm. Biopharm. 2000, 50, 27-46.

[8] Peppas, N. A.; Khare, A. R. Adv. Drug Delivery Rev. 1993, 11, 1-35.

[9] Chujo, Y.; Sada, K.; Saegusa, T. Macromolecules 1993, 26, 6320-6323.

[10] Fiore, G. L.; Klinkenberg, J. L.; Pfister, A.; Fraser, C. L. Biomacromolecules 2009, 10, 128-133.

[11] Fiore, G. L.; Klinkenberg, J. L.; Fraser, C. L. Macromolecules 2008, 41, 9397-9405. 
[12] Vermonden, T.; de Vos, W. M.; Marcelis, A. T. M.; Sudholter, E. J. R. Eur. J. Inorg. Chem. 2004, 2847-2852.

[13] Vermonden, T.; van Steenbergen, M. J.; Besseling, N. A. M.; Marcelis, A. T. M.; Hennink, W. E.; Sudholter, E. J. R.; Stuart, M. A. C. JACS 2004, 126, 15802-15808.

[14] Wild, A.; Hornig, S.; Schlutter, F.; Vitz, J.; Friebe, C.; Hager, M. D.; Winter, A.; Schubert, U. S. Macromol. Rapid Commun. 2010, 31, 921-927.

[15] Chiper, M.; Hoeppener, S.; Schubert, U. S.; Fustin, C. A.; Gohy, J. F. Macromol. Chem. Phys. 2010, 211, 2323-2330.

[16] Buwalda, S. J.; Dijkstra, P. J.; Calucci, L.; Forte, C.; Feijen, J. Biomacromolecules 2010, 11, 224-232.

[17] Calucci, L.; Forte, C.; Buwalda, S. J.; Dijkstra, P. J.; Feijen, J. Langmuir 2010, 26, 12890-12896.

[18] Piepenbrock, M. O. M.; Lloyd, G. O.; Clarke, N.; Steed, J. W. Chem. Rev. 2010, 110, 1960-2004.

[19] Tanodekaew, S.; Godward, J.; Heatley, F.; Booth, C. Macromol. Chem. Phys. 1997, 198, 3385-3395.

[20] Constable, E. C. Adv. Inorg. Chem. 1986, 30, 69-121.

[21] Barbucci, R.; Leone, G.; Magnani, A.; Montanaro, L.; Arciola, C. R.; Peluso, G.; Petillo, O. J. Mater. Chem. 2002, 12, 3084-3092.

[22] Hiemstra, C.; Zhong, Z.; Li, L. B.; Dijkstra, P. J.; Feijen, J. Biomacromolecules 2006, 7 , 2790-2795.

[23] Hong, H. Y.; Mai, Y. Y.; Zhou, Y. F.; Yan, D. Y.; Cui, J. Macromol. Rapid Commun. 2007, 28, 591-596. 


\section{Summary}

Hydrogels are hydrophilic polymer networks that are able to retain large amounts of water. They generally exhibit excellent biocompatibility and are accordingly of interest for biomedical and pharmaceutical applications such as systems for the controlled delivery of biologically active agents. Hydrogels are networks that can be physically crosslinked by non-covalent interactions and/or chemically crosslinked by covalent bonds. Both approaches have been used in recent years for the preparation of hydrogels that can be applied under physiological conditions. Of special interest are biodegradable injectable hydrogels, also called "in situ" forming hydrogels. These gels are formed at the injection site after the introduction of fluid precursors in a minimally invasive manner. "In situ" forming hydrogels offer several advantages over implantation of pre-shaped devices. There is no need for surgical procedures and their initially flowing nature ensures proper shape adaptation as well as a good fit with the surrounding tissue. Moreover, cells or biologically active agents can be easily incorporated in the injectable fluid.

Hydrogel based controlled drug delivery systems can potentially be used to address a number of issues that are encountered in conventional drug delivery, such as poor control of local or systemic drug concentration and the low solubility of many therapeutic agents in biological fluids. Most physically and chemically crosslinked biodegradable hydrogels that have been applied as controlled drug delivery systems are based on linear amphiphilic block copolymers of poly(ethylene glycol) (PEG) and aliphatic polyesters. Star block copolymers offer various advantages over linear polymers, such as increased solubility in aqueous media and a higher concentration of functional end groups. Starting from an 8-armed PEG block we designed and explored different block copolymers composed of outer poly(lactide) (PLA) or poly(trimethylene carbonate) (PTMC) blocks, for the preparation of physically or chemically crosslinked injectable hydrogels. The gelation and hydrogel degradation mechanisms involved were investigated in detail and their potential as systems for the controlled delivery of biologically active agents was evaluated.

In Chapter 2 physical and chemical crosslinking methods are reviewed that have been applied for the synthesis of PEG based hydrogels for biomedical applications. The emphasis is on PEGPLA block copolymer hydrogels that can be formed in situ. The examples discussed in this chapter represent some of the approaches that have been used to synthesize physically or chemically crosslinked hydrogel networks that may be used in biomedical and pharmaceutical applications. It was concluded that the development of new materials and a deeper understanding of underlying gelation mechanisms will inevitably result in an even greater role for hydrogels in biomedical applications.

Preferential hydrolysis of linking ester groups between the PEG and PLA blocks is generally accepted to cause a rapid hydrolytic degradation of PEG-PLA type hydrogels. To avoid this 
preferential hydrolysis the synthesis of 8-armed PEG-PLA star block copolymers linked by an amide group between the PEG core and the PLA blocks (PEG-(NHCO)-(PLA) 8 ) was investigated and the results are described in Chapter 3. It was shown that in PEG-(NHCO)-(PLA) 8 hydrogels degradation only takes place via hydrolysis of ester groups in the PLA chains, affording materials that are more slowly degrading than PEG-(OCO)-(PLA) 8 hydrogels. The PEG-(NHCO)-(PLA) 8 star block copolymers in water had a low critical gel concentration (CGC) and exhibited a temperature dependent phase behavior. Furthermore, these copolymers had a larger gel window compared to PEG-(OCO)-(PLA) 8 block copolymers. The research described in Chapter 3 was extended by preparing the corresponding enantiomeric PEG-PDLA and PEG-PLLA star block copolymers and studying the effect of stereocomplexation on the gelation behavior and hydrogel properties (Chapter 4). When $6 \mathrm{w} / \mathrm{v} \%$ PEG-(NHCO)-(PDLA 13$)_{8}$ and PEG-(NHCO)-(PLLA 13$)_{8}$ aqueous solutions were mixed, a stereocomplexed hydrogel formed within 1 minute. Compared to single enantiomer hydrogels of similar concentration, stereocomplexed PEG-(NHCO)-(PLA) 8 hydrogels exhibited enhanced mechanical properties and an improved in vitro stability. Rheology and nuclear magnetic resonance (NMR) experiments revealed that formation of stereocomplexes is facilitated at higher temperatures, possibly due to rearrangement in the aggregates thereby exposing more PLA units available for stereocomplexation. The formed gels did not return to their sol state upon cooling due to the presence of highly stable semi-crystalline stereocomplexed PLA domains. Stereocomplexed PEG-(NHCO)-(PLA) 8 hydrogels were more stable towards hydrolysis than stereocomplexed PEG-(OCO)-(PLA) ${ }_{8}$ hydrogels. In an initial study it was shown that the release of a small protein like lysozyme from the stereocomplexed gels for 30 days was diffusion controlled. In Chapter 5 the synthesis of 8-armed PEG-PTMC star block copolymer (PEG-(PTMC 9$)_{8}$ ) by metal-free ring opening polymerization of trimethylene carbonate (TMC) is reported. Although dye solubilization experiments, NMR and dynamic light scattering clearly indicated the presence of aggregates in aqueous dispersions of the copolymers, no physical gelation was observed up to high polymer concentrations. A detailed study towards the aggregation behavior suggested that the residence time of the PTMC blocks in the hydrophobic regions is too short to obtain a stable network. PEG-(PTMC 9$)_{8}$ was functionalized with acrylate $(\mathrm{AC})$ end groups and photopolymerized. UV irradiation of dilute $(<5 \mathrm{w} / \mathrm{v} \%)$ PEG-(PTMC 9$)_{8}-\mathrm{AC}$ dispersions resulted in covalently crosslinked PEG-PTMC nanoparticles, whereas more concentrated dispersions yielded chemically crosslinked PEG-PTMC hydrogels. Photocrosslinked PEG-PTMC hydrogels exhibited excellent in vitro stability with only $10 \%$ mass loss after 6 weeks and showed no toxicity towards chondrocytes.

Chemically crosslinked PEG-PLA hydrogels prepared by a Michael addition reaction between multi-armed PEG-(SH) and acrylated PEG-PLA star block copolymers (PEG-(PLA $)_{8}$-AC) are described in Chapter 6. The mechanical and degradation properties of these hydrogels could be 
tuned by changing the functionality of the PEG-(SH)n . Mixing PEG-(PLA $\left.)_{8}\right)_{8}-\mathrm{AC}$ and PEG-(SH) 8 solutions $(5 \mathrm{w} / \mathrm{v} \%$ in PBS) resulted in the formation of a hydrogel in less than a minute, which quickly reached a high storage modulus of $17 \mathrm{kPa}$. Degradation times increased with crosslink density and varied from 3 days for PEG-(PLA 12$)_{8}$-AC / PEG-(SH) $)_{2}$ hydrogels to several months for PEG-(PLA 12$)_{8}$-AC / PEG-(SH) hydrogels. The degradation of the hydrogels had a significant effect on the protein release behavior. Lysozyme and albumin were released from the highly crosslinked PEG-(PLA 12$)_{8}$-AC / PEG-(SH) 8 hydrogels for 4 weeks mainly by diffusion. The release of lysozyme from PEG-(PLA 12$)_{8}$-AC / PEG-(SH) 2 and PEG-(PLA 12$)_{8}-\mathrm{AC} /$ PEG-(SH) 4 hydrogels was significantly faster with complete release in 3 and 12 days, respectively. The release was governed by a combination of diffusion and degradation. Importantly, the released lysozyme retained $100 \%$ activity. In Chapter 7 PEG-PLA type hydrogels are reported that can be formed by physical crosslinking through stereocomplexation followed by photopolymerization. In this way enantiomeric PEG-(PDLA 12$)_{8}$-AC and PEG-(PLLA 12$)_{8}$-AC star block copolymers afforded highly robust and stable hydrogels. The release properties of the photopolymerized gels were evaluated using lysozyme, albumin and rhodamine B as model compounds. A first order release of albumin and lysozyme over a period of 10 days was observed. The released lysozyme lost some of its enzymatic activity possibly due to denaturation of the protein by UV irradiation and radicals generated during the photocrosslinking of the gel. The release of rhodamine $\mathrm{B}$ from the stereocomplexed and photocrosslinked PEG-PLA hydrogels was characterized by an initial burst followed by a degradation dependent release during several months. In Chapter $\mathbf{8}$ an initial study is presented towards the aqueous solution behavior and thermo-reversible gelation properties of pyridine end functionalized PEG-PLA star block copolymers (PEG-(PLLA)s-py) in the presence of coordinating transition metal ions. In aqueous solutions the macromonomers self-assembled into micelles and micellar aggregates at low concentrations and formed physically crosslinked, thermoreversible hydrogels above a CGC of $8 \mathrm{w} / \mathrm{v} \%$. The presence of $\mathrm{Mn}(\mathrm{II})$ ions resulted in increased aggregate dimensions, a larger gel window and increased in vitro stability due to the coordination of pyridine ligands around the metal centers, thereby introducing additional crosslinks. Importantly, the stabilizing effect of metal-ligand coordination was noticeable at very low, non-toxic metal ion concentrations. 


\section{Samenvatting}

Hydrogelen zijn hydrofiele polymeer netwerken die grote hoeveelheden water kunnen opnemen. Ze zijn over het algemeen zeer biocompatibel en dientengevolge erg interessant voor biomedische en farmaceutische toepassingen zoals systemen voor de gecontroleerde afgifte van biologisch actieve stoffen. Deze netwerken kunnen of fysisch gecrosslinkt door non-covalente interacties en/of chemisch gecrosslinkt zijn door covalente bindingen. Beide methoden zijn gebruikt voor het maken van hydrogelen die onder fysiologische condities toegepast kunnen worden. Bijzonder interessant zijn biodegradeerbare en injecteerbare hydrogelen, die ook wel "in situ" vormende hydrogelen worden genoemd. Deze gelen vormen zich op de plaats van injectie uit polymeeroplossingen door het instantaan vormen van fysische of chemische crosslinks. "In situ" vormende hydrogelen bieden diverse voordelen ten opzichte van het implanteren van voorgevormde hydrogelen. Chirurgische ingrepen zijn niet nodig en het initieel vloeibare karakter zorgt voor een goede aansluiting met omliggend weefsel. Bovendien kunnen cellen of biologisch actieve stoffen gemakkelijk worden geïncorporeerd in de injecteerbare vloeistof.

Hydrogel systemen voor de gecontroleerde afgifte van medicijnen kunnen gebruikt worden om een aantal nadelen van hedendaagse medicijn afgifte systemen te ondervangen zoals beperkte controle over de lokale of systemische concentratie van de therapeutische stof en/of de algemeen beperkte oplosbaarheid in biologische vloeistoffen. De meeste fysisch en chemisch gecrosslinkte biodegradeerbare hydrogelen die zijn toegepast als systemen voor de gecontroleerde afgifte van medicijnen zijn gebaseerd op lineaire amfifiele blokcopolymeren van poly(ethyleen glycol) (PEG) en alifatische polyesters. Ten opzichte van lineaire polymeren bieden stervormige blokcopolymeren als uitgangsmateriaal voor hydrogelen diverse voordelen, zoals een betere oplosbaarheid in water en fysiologische buffers en een hogere concentratie functionele eindgroepen. Op basis hiervan zijn vanuit een centraal 8-armig PEG polymeer door ring-opening polymerisatie van lactide of trimethyleencarbonaat 8-armige PEG-(PLA) 8 en PEG-(PTMC) 8 blokcopolymeren gesynthetiseerd. De gelering en degradatie mechanismen van de hieruit verkregen hydrogelen zijn onderzocht en hun bruikbaarheid als systemen voor de gecontroleerde afgifte van biologisch actieve stoffen geëvalueerd.

In Hoofdstuk 2 wordt een overzicht gegeven van methoden die gebruikt zijn voor het fysisch en chemisch crosslinken van op PEG gebaseerde hydrogelen voor biomedische toepassingen. De nadruk in dit overzicht is gelegd op PEG-PLA blokcopolymeren die in situ gevormd kunnen worden. Met een aantal voorbeelden zijn deze algemene methoden geïllustreerd. Op basis van de huidige stand van zaken kan geconcludeerd worden dat een verdere ontwikkeling van nieuwe materialen voor injecteerbare hydrogelen en fundamentele kennis van de gelering mechanismen belangrijk zijn voor nieuwe biomedische en farmaceutische toepassingen. 
De preferentiële hydrolyse van de ester groepen tussen PEG en PLA blokken, een direct resultaat van ring-opening polymerisatie van lactonen aan de PEG hydroxyl eindgroepen, wordt doorgaans als oorzaak gezien voor de snelle hydrolytische degradatie van PEG-(OCO)-PLA type hydrogelen, waaronder ook die gebaseerd op 8-armige PEG-PLA stervormige blokcopolymeren (PEG-(OCO)$\left.(\mathrm{PLA})_{8}\right)$. Om deze preferentiële hydrolyse te voorkomen werden 8-armige PEG-PLA stervormige blokcopolymeren met een amide groep tussen de PEG kern en de PLA blokken (PEG-(NHCO)$\left.(\mathrm{PLA})_{8}\right)$ onderzocht en de resultaten zijn in Hoofdstuk 3 beschreven. Er werd aangetoond dat in PEG-(NHCO)-(PLA) ${ }_{8}$ hydrogelen degradatie alleen plaatsvindt via hydrolyse van ester groepen in de PLA ketens, waardoor deze gelen dus langzamer degraderen dan PEG-(OCO)-(PLA) 8 hydrogelen. Bovendien hadden de PEG-(NHCO)-(PLA) $)_{8}$ stervormige blokcopolymeren een lage kritische gel concentratie en vertoonden temperatuur afhankelijk gelering gedrag dat ze geschikt maakt als een injecteerbaar hydrogel systeem. Het onderzoek als beschreven in Hoofdstuk 3 was de basis voor een studie naar de stereocomplexatie van mengsels van enantiomere PEG-PDLA en PEG-PLLA stervormige blokcopolymeren en het effect op het gelering gedrag en de eigenschappen van de verkregen hydrogelen (Hoofdstuk 4). Na het mengen van 6 w/v \% PEG-(NHCO)$\left(\mathrm{PDLA}_{13}\right)_{8}$ en PEG-(NHCO)-(PLLA 13$)_{8}$ waterige oplossingen ontstond binnen 1 minuut een "stereocomplex hydrogel". Vergeleken met hydrogelen als beschreven in Hoofdstuk 3 die waren gemaakt met één enantiomeer, vertoonden de stereocomplex PEG-(NHCO)-(PLA) 8 hydrogelen een hoge in vitro stabiliteit. Met reologische en kernspin resonantie (NMR) experimenten is aangetoond dat er bij hogere temperaturen een toename is in de vorming van stereocomplexen, mogelijk door herordening in en tussen de micellaire en grotere aggregaten waardoor meer PLA ketens beschikbaar komen voor stereocomplexatie. De gevormde gelen vertoonden uiteindelijk geen temperatuur afhankelijke gel-sol overgang meer door de vorming van zeer stabiele semi-kristallijne stereocomplex PLA domeinen en bleven in de gel fase. Stereocomplex PEG-(NHCO)-(PLA) 8 hydrogelen waren beter bestand tegen hydrolytische degradatie dan stereocomplex PEG-(OCO)$(\mathrm{PLA})_{8}$ hydrogelen. In een initiële studie werd aangetoond dat de afgifte van een klein eiwit als lysozyme vanuit de stereocomplex hydrogelen gedurende 30 dagen diffusie gecontroleerd is.

In Hoofdstuk 5 wordt de synthese beschreven van 8-armige PEG-PTMC stervormige blokcopolymeren (PEG-(PTMC $\left.)_{8}\right)$ door middel van zuurgekatalyseerde ring-opening polymerisatie van trimethyleencarbonaat (TMC). Met deze methode wordt het gebruik van metaalbevattende katalysatoren, vaak gebruikt in de ring-opening polymerisatie van TMC, vermeden. Hoewel met o.a. NMR en dynamische licht verstrooiing duidelijk het vormen van aggregaten bij lage concentratie kon worden aangetoond treedt geen fysische gelering op bij hoge concentraties. Verder onderzoek naar het aggregatie gedrag liet een hoge mobiliteit van de PTMC blokken in de hydrofobe domeinen zien, en er is gesuggereerd dat deze hoge mobiliteit de vorming van stabiele netwerken verhindert. Omdat gelering door fysisch crosslinken niet mogelijk was werden de PEG- 
$\left(\mathrm{PTMC}_{9}\right)_{8}$ ster polymeren gefunctionaliseerd met acrylaat $(\mathrm{AC})$ eindgroepen en daarna gefotopolymeriseerd. UV bestraling van verdunde $(<5 \mathrm{w} / \mathrm{v} \%)$ PEG-(PTMC 9$)_{8}-\mathrm{AC}$ dispersies resulteerde in chemisch (covalent) gecrosslinkte PEG-PTMC nanodeeltjes, terwijl bij hogere concentraties chemisch gecrosslinkte PEG-PTMC hydrogelen werden verkregen. Fotogecrosslinkte PEG-PTMC hydrogelen vertoonden een uitstekende in vitro stabiliteit met slechts $10 \%$ massa verlies na 6 weken.

Chemisch gecrosslinkte PEG-PLA hydrogelen gemaakt via een Michael additie reactie tussen multi-arm PEG-(SH) en geacryleerde PEG-PLA stervormige blokcopolymeren (PEG-(PLA $)_{8}$-AC) worden beschreven in Hoofdstuk 6. De mechanische en degradatie eigenschappen van deze hydrogelen zijn afhankelijk van het aantal armen van de PEG-( $\mathrm{SH})_{\mathrm{n}}$ macromonomeren. Het mengen van PEG-(PLA $)_{8}$-AC en PEG-(SH) 8 oplossingen ( $5 \mathrm{w} / \mathrm{v} \%$ in PBS) resulteerde in de vorming van een hydrogel in minder dan een minuut, met een hoge opslag modulus van $17 \mathrm{kPa}$. De degradatie tijden namen toe met de crosslink dichtheid en varieerden van 3 dagen voor PEG-(PLA 12$)_{8}$-AC / PEG-(SH) $)_{2}$ hydrogelen tot enkele maanden voor PEG-(PLA $\left.{ }_{12}\right)_{8}-\mathrm{AC} / \mathrm{PEG}-(\mathrm{SH})_{8}$ hydrogelen. De degradatie had een grote invloed op de afgifte van geïncorporeerde eiwitten. Lysozyme en albumine werden afgegeven vanuit de sterk gecrosslinkte PEG-(PLA 12$)_{8}$-AC / PEG-(SH) 8 hydrogelen gedurende 4 weken, voornamelijk door middel van diffusie. De afgifte van lysozyme vanuit PEG$\left(\mathrm{PLA}_{12}\right)_{8}$-AC / PEG-(SH) $)_{2}$ en PEG-(PLA 12$)_{8}$-AC / PEG-(SH) ${ }_{4}$ hydrogelen was met respectievelijk 3 en 12 dagen aanzienlijk sneller. Deze afgifte werd bepaald door een combinatie van diffusie en degradatie. Een belangrijk aspect was dat het lysozyme zijn enzymatische activiteit volledig behield. In Hoofdstuk 7 worden PEG-PLA type hydrogelen beschreven die worden gevormd door fysische crosslinking via stereocomplexatie, gevolgd door fotopolymerisatie. Enantiomere PEG$\left(\mathrm{PDLA}_{12}\right)_{8}$-AC en PEG-(PLLA 12$)_{8}$-AC stervormige blokcopolymeren leverden hydrogelen op met een hoge opslagmodulus. De afgifte van lysozyme, albumine en rhodamine B als model stoffen vanuit de gefotopolymeriseerde hydrogelen werd daarna onderzocht. Er werd een eerste orde afgifte van albumine en lysozyme gedurende 10 dagen waargenomen. De lysozyme had een deel van haar enzymatische activiteit verloren, mogelijk als gevolg van denaturatie door UV bestraling en radicalen die ontstaan zijn tijdens het fotocrosslinken. De afgifte van rhodamine $B$ vanuit de fotogecrosslinkte stereocomplex PEG-PLA hydrogelen werd gekenmerkt door een initiële snelle afgifte gevolgd door een degradatie afhankelijke afgifte gedurende enkele maanden. Ten slotte zijn in een initiële studie de macromoleculaire thermo-reversibele gelering eigenschappen van de eerder beschreven PEG-PLA blokcopolymeren gecombineerd met de supramoleculaire eigenschappen van pyridine overgangsmetaal ion complexen (Hoofdstuk 8). Na eind functionalisering van PEG$\left(\mathrm{PLLA}_{12}\right)_{8}$ met pyridine eindgroepen tot PEG-(PLLA 12$)_{8}$-py is het thermo-reversibele gelering gedrag in water onderzocht. De blokcopolymeren vormden micellen en micellaire aggregaten bij lage concentraties en fysisch gecrosslinkte gelen boven een kritische gel concentratie van $8 \mathrm{w} / \mathrm{v} \%$. 
In de aanwezigheid van $\mathrm{Mn}(\mathrm{II})$ ionen zijn de aggregaten groter en is de kritische gel concentratie lager. Door de vorming van coördinatie complexen van de pyridine liganden met de metaal ionen worden naast de crosslinks gevormd door de hydrofobe PLA domeinen extra crosslinks geïntroduceerd wat leidt tot een hogere in vitro stabiliteit van de gelen. Dit stabiliserende effect van metaal-ligand coördinatie in hydrogelen is al aanwezig bij zeer lage metaal ion concentraties hetgeen belangrijk is in biomedische toepassingen. 


\section{Acknowledgements}

Bijna 4.5 jaar na de start van mijn promotietraject is het tijd om terug te blikken op een geweldige periode. Uiteraard zijn er momenten geweest waarop ik dacht: 'waar ben ik aan begonnen', maar die zijn gelukkig op de vingers van 1 hand te tellen. Wat overheerst is het gevoel dat ik dik 4 jaar bevoorrecht ben geweest om een voortgezette opleiding tot wetenschapper te kunnen combineren met onderzoek in de fascinerende en relevante biomedische wereld. Niet alleen het werk zelf, maar ook de academische omgeving in het algemeen en die in Twente in het bijzonder zijn erg goed bevallen. Het is dan ook nodig om een flink aantal mensen te bedanken.

Als eerste mijn co-promotor Piet Dijkstra. Piet, ik weet nog goed dat ik als afstudeerder bezig was op lab ZH 283 met het bepalen van een zwelgraad van een hydrogel toen jij binnenkwam en vroeg of ik al wist wat ik na mijn studie wilde gaan doen. Toen ik daarop negatief antwoordde, liet jij de term 'promoveren' vallen en daar hoefde ik niet lang over na te denken. Bijna 4.5 jaar later kan ik zeggen dat ik geen spijt heb gehad van die beslissing. Dank voor deze kans, de soepele begeleiding en de mogelijkheid altijd even binnen te lopen. Ook al liep ik soms met een overvol en/of verward hoofd weer terug, uiteindelijk bleek je advies altijd nuttig. Ook heb ik nog altijd uitstekende herinneringen aan de werkbezoeken aan Pisa en het congres in Viareggio die een flinke dosis 'La Dolce Vita' ademden. Leuk dat jij nu ook een toga mag dragen bij promoties, ik ben benieuwd of de langverwachte Chinese versie al klaar is...

Natuurlijk mag mijn promotor hier niet onbenoemd blijven. Professor Feijen, het is een voorrecht geweest om met $\mathrm{u}$ te mogen werken. Ik denk met bewondering terug aan de werkbesprekingen waarbij mijn problemen en vragen door $u$ aangepakt werden door middel van een mix van kennis, inzicht en ervaring. Maar ook buiten de Universiteit Twente, bijvoorbeeld op congres of op studiereis, was het waarlijk een genoegen om tijd met u door te brengen vanwege uw humor, geweldige verhalen, sociale inzicht en eruditie.

Professor Engbersen en Dr. André Poot, dank voor de waardevolle wetenschappelijke discussies vanuit de organisch-chemische respectievelijk biochemische hoek tijdens de vakgroepvergaderingen.

I would also like to thank my collaborators Dr. Claudia Forte and Dr. Lucia Calucci. To date we managed to publish 4 papers together in excellent journals. I am still impressed by the enormous amount of relevant data and conclusions you were able to produce at short timescales. Thanks for the hospitable receptions and fruitful discussions in Pisa in April 2008 and November 2009. Dr. Forte, I am looking forward to greet you as a very learned opponent during my $\mathrm{PhD}$ defense.

I would also like to thank Prof. Dr. G. W. Grijpma, Prof. Dr. R. G. H. Lammertink, Prof. Dr. W. E. Hennink, Prof. Dr. Ir. J. C. M. Van Hest and Prof. Dr. C. Jérôme for their willingness to participate in my graduation committee and for their careful evaluation of my thesis. 
Dit proefschrift had er nooit gelegen zonder de hulp van de vaste krachten binnen de PBM vakgroep. Karin, dank voor al het geregel en geadministreer, zonder jou zouden je huidige 2 vakgroepen zonder twijfel volledig in de soep lopen. Ook de gezellig praatjes, als ik weer eens langskwam met een of ander te scannen document, heb ik altijd zeer gewaardeerd. Zlata, ook bij jou ging je kantoor binnenlopen zelden zonder een babbeltje over vakanties of wat dan ook. Dankzij jou kon ik lekker doorwerken op het lab zonder me zorgen te hoeven maken over bestellingen of algemene benodigdheden. Hetty, dank voor de soms broodnodige hulp op het gebied van ICT. Marc, je hebt enkele fikse batches PEG-PLA blokcopolymeren gesynthetiseerd tegen het eind van mijn project, in een tijd dat ik het erg druk had met het afronden van allerlei losse eindjes. Dank daarvoor en voor de praktische begeleiding van enkele studenten, een discipline waar je ijzersterk in bent.

Naast het uitvoeren van werkzaamheden op het laboratorium, heeft mijn hart ook altijd gelegen bij het begeleiden en lesgeven van mensen. Ik ben daarom blij dat er tijdens mijn promotie 5 studenten zijn langsgekomen voor het uitvoeren van een (afstudeer)project. Björn de Wagenaar, jouw werk is de aanzet geweest tot Hoofdstuk 6 van dit proefschrift. Dank voor je inzet en je aanstekelijke enthousiasme, zelfs als er weer eens een batch PEG thiolen gemaakt moest worden (enkele lades in ZH 281 herinneren daar nog steeds aan (-)). Het verbaast me niets dat je ook bent gaan promoveren, succes ermee! Laura Badia Perez, you came to Enschede as an Erasmus Exchange student. I feared somewhat for your working ethos, since Erasmus students have a certain reputation $:$, especially Spanish ones, but you managed to combine partying hard and working hard and contributed significantly to Chapter 5 which was published in Biomacromolecules. Thanks for your efforts and for bringing your sunny Spanish character to ZH 281! Laura Grana Suarez, the start of your Master assignment went a little bumpy, but soon you turned out to be a (Seat) Diesel engine and you kept on going and going, even during the evenings and in the weekends. In the end you produced an impressive amount of interesting results. Unfortunately your project ended just before my $\mathrm{PhD}$ thesis had to be submitted and your work could not be included any more. Good luck with your own $\mathrm{PhD}$ project in the MnF group! Marijke de Graaff, jouw Bachelor opdracht is de opmaat geweest voor Hoofdstuk 8. Dank voor je doorzettingsvermogen (je gaf 'in situ' vormende gel een geheel nieuwe dimensie :-) - we zijn er nog steeds niet achter wat er chemisch gezien met de batch gecarboxyleerde PEG-PLA gebeurd kan zijn...) en je vrolijkheid op het laboratorium. Succes met de rest van je studie! Maarten Blokzijl, toen je langskwam voor een Bachelor opdracht herinnerde ik me je nog van het $1^{\mathrm{e}}$ jaars practicum Chemie en Biomaterialen als één van een select groepje erg positief ingestelde studenten. Ook al waren de resultaten soms niet waar we op hoopten en af en toe wat ambigue, je liet je niet uit het veld slaan en bleef vol overgave en discipline lekker bezig op het lab. Je draaide ook sociaal volop mee in de vakgroep (jij, Jos en ik 
hebben toch maar mooi de $1 / 8$ triathlon binnen de 2 uur afgelegd $(\dot{)})$ en uiteindelijk hebben we een mooie proof-of-principle kunnen aantonen. Succes met je Master!

Niet alleen is de afgelopen 5 jaar een leerzame periode geweest in velerlei opzichten, het is ook een ongelooflijk gezellige tijd geweest. Het was geweldig om elke dag door te kunnen brengen met gelijkgestemde mensen die niet alleen collega's waren, maar in veel gevallen ook vrienden zijn geworden. Ik zal de herinneringen aan de talloze uitjes, etentjes, borrels, triathlons, studiereizen, bowling- squash- en badminton-avondjes, afstudeer- promotie- en oratiefeestjes, congressen, UT sportdagen, en niet te vergeten de zeiltrip op de Res Novae nog lang koesteren. Iedereen heel uitgebreid bedanken zou betekenen dat mijn proefschrift Gregoriaanse proporties zou aannemen, maar enkele personen wil ik absoluut noemen, te beginnen met mijn paranimfen. Jos, jij liep al bij PBM rond toen ik ruim 5 jaar geleden, toen nog in good old $\mathrm{CT}$, als afstudeerder begon, maar we leerden elkaar pas echt goed kennen toen jij ook ging promoveren en mijn kantoorgenoot werd in $\mathrm{ZH}$ 233. We hebben menig gezellig uurtje doorgebracht, al dan niet met een lantaarnpaalverplaatsende St. Bernardus Abt 12 in de hand, en konden elkaar ook wetenschappelijk prima aanvullen en -voelen (en opbeuren als er weer eens een experiment dramatisch verliep (:)). Tof dat je me ter linker zijde wilt flankeren tijdens mijn verdediging. Robert, jou ken ik al meer dan 20 jaar, en hoewel we na 12 jaar in Apeldoorn op school te hebben gezeten in verschillende steden gingen studeren, bleven we elkaar regelmatig zien in Apeldoorn, Amsterdam of Enschede om bij te praten en een biertje te drinken. We delen een voorliefde voor wolkenkrabbers en andere hoge constructies en in dat licht bezien waren de Corsendonck op de Tour Montparnasse in Parijs en de König bovenop de in onbruik geraakte cokesfabriek bij Zeche Zollverein in Essen letterlijk en figuurlijk hoogtepunten. Ik waardeer het zeer dat je me op 8 december ter rechter zijde wilt bijstaan.

Niels, we zijn 3 jaar lang buren geweest in ZH 281 en hebben ook buiten het lab veel tijd met elkaar doorgebracht. Ik heb uitstekende herinneringen aan de tripjes samen met Jos naar Barcelona en Edinburgh, waarbij die laatste trip getypeerd kan worden als een kletsnatte trip met de droogste persoon die ik ooit ben tegengekomen. Baco's hebben we zowaar niet gedronken in Edinburgh, maar we hebben genoeg ander vocht zien stromen. Janine, ik denk met veel plezier terug aan ons congresbezoek in Salt Lake City en de aansluitende roadtrip, met die geweldige National Parks en de gekkigheid in Las Vegas. Ik zal verder maar niet uitweiden over een zekere small block 6.2L V8 (-). Respect dat je met je 9-tot-5 ritme toch nog dik binnen 4 jaar klaar bent, daarbij in mooie bladen publicerend. Siggi, ik ben zelden zo'n warme en sociale persoonlijkheid als de jouwe tegengekomen. Het was gezellig om 1.5 jaar overburen te zijn boven de Klanderij, ook al dacht je zoon daar tenminste 1 avond iets anders over $:$. Lekker dat je samen met Bart-Jan en Jesse een plekje hebt gevonden in het fraaie Münster! Hans, jou ken ik al vanaf de middelbare school. Bijzonder dat we al vanaf 1993 vrijwel parallel onze opleidingen hebben genoten met eerst het Gymnasium in Apeldoorn, daarna CT aan de UT en tenslotte promotie binnen dezelfde vakgroep. 
Bewonderenswaardig hoeveel energie jij naast je werk nog hebt voor allerlei andere dingen, niet in de laatste plaats het opknappen van een huis uit 1903. Veel geluk met Kyra in Lelystad, Groningen of waar jullie ook maar terechtkomen! Andries, jij gaat vast en zeker verder met je het waarmaken van je geambieerde academische carrière. Als er iemand geschikt voor is, ben jij het wel. Gelukkig weet je daarbij oog te houden voor Belangrijke Zaken als keihard bierdrinken, al dan niet gevolgd door het nuttigen van $\mathrm{Ku}$ Yo Luk-achtige deegballen gevuld met kipdelen. Kim, of er nou een karafje rosé in je zit of niet, je aanwezigheid zorgt altijd voor een flinke portie vrolijkheid. Sjoerd, naast de 2 supergezellige, door jou georganiseerde pokeravonden vond ik ook de paar door mij bijgewoonde zondagavond=speciaalbieravonden erg geslaagd, ook al dacht ik daar de volgende morgen steevast heel anders over. Erwin Zant, nog even doorAIOen en dan via PLem bij DSM gaan werken, dan komt die Impreza STi of door Oettinger gekietelde R32 er wel (). The following people definitely should be mentioned as well: Suzanne S., Suzanne B., Leonie, Joost, Maureen, Edit, Wilma, Xiaofeng, Aysegul, Erhan (een zonnige tijd toegewenst aan de Westcoast!), Federico, Gert-Jan (ski bike al binnen? (;), Gregory, Henriette, Ingrid (dank voor je prima begeleiding tijdens mijn afstuderen, dat jaar heeft me uiteindelijk enthousiast gemaakt voor de wetenschap), Jolanda, Martin, Peter van E., Peter B., Sander, Sandra T., Sandra de V., Anne Corine, Sri Dewi, Mark P. (drankje bij de Buurvrouw op 9 december om 04:00? ()), Dimitrios, Lydia, Anita, Sebastien, Suvi, Frits (volgens mij help jij de VriMiBo erbovenop), Shariar, Marlon, Erwin N., Xiaolin, Guoying Si, Bona, Mark ten B., Jung Seok, Rong, Lanti, Zhao Di, Alfredo, Ferry, Marloes, Vincent, Arkadi, Kasia, and all other (former) colleagues, students and so on!

Natuurlijk moet ik ook de mensen bedanken die ik al het langste ken. Pa en ma, dank voor de onvoorwaardelijke steun. Waarschijnlijk zegt een gepast citaat meer dan een hele alinea tekst: dimidium facti qui coepit habet. Dank daarvoor!

Time flies when you are having fun. Er zijn ruim 1600 dagen verstreken sinds de start van mijn promotie op 1 juni 2007, maar het lijken er slechts 160. Ook al heb ik me steeds voorgenomen om geen clichés te gebruiken in mijn dankwoord, in de laatste alinea ga ik alsnog voor de bijl. Hoogste tijd dus om te stoppen. Het is mooi geweest! 


\section{Curriculum Vitae}

Sytze Jan Buwalda was born on Augustus 21 ${ }^{\text {st }}, 1981$ in Apeldoorn, the Netherlands. He obtained his 'Gymnasium' diploma in 1999 at the Stedelijk Gymnasium Apeldoorn. In the same year he started studying Chemical Engineering at the University of Twente in Enschede, the Netherlands with a major in Biomedical Material Science. In 2004 he performed his internship at DSM Composite Resins in Zwolle, the Netherlands, conducting research on composites based on photocrosslinkable polyester resins for the restoration of damaged sewer systems. He received his Master of Science degree (Dutch: ingenieur, Ir.) in the Polymer Chemistry and Biomaterials group led by Prof. Dr. J. Feijen at the University of Twente in 2007. His master project was entitled 'Biodegradable chemically crosslinked PEG-PLLA hydrogels for drug delivery purposes' and was performed under daily supervision of Dr. Ir. I. W. Velthoen. He started as a PhD student in the same research group in the summer of 2007 under the supervision of Prof. Dr. J. Feijen and Prof. Dr. P. J. Dijkstra. The PhD research was entitled 'Hydrogels based on amphiphilic PEG star block copolymers'. The results of this research are described in this $\mathrm{PhD}$ thesis. 